CERN-TH/96-111

$22 / 04 / 1996$

\title{
Minimal Supersymmetric Standard Model Higgs rates and backgrounds in ATLAS
}

\author{
Elżbieta Richter-Wąs ${ }^{1}$ \\ CERN, Theory Division, 1211 Geneva 23, Switzerland \\ Institute of Computer Science, Jagellonian University, \\ 30-059 Kraków, ul. Reymonta 4, Poland \\ Institute of Nuclear Physics, Cracow, Poland \\ Daniel Froidevaux, Fabiola Gianotti, Luc Poggioli \\ CERN, PPE Division, 1211 Geneva 23, Switzerland \\ Donatella Cavalli, Silvia Resconi \\ Physics Department, Milan University and I.N.F.N., \\ I-20133 Milan, Via Celoria 16, Italy
}

\begin{abstract}
This study presents an overview of the Minimal Supersymmetric Standard Model Higgs rates, backgrounds and significances expected for the ATLAS detector at LHC. Since the submission of the ATLAS Technical Proposal (TP), much has changed in the overall picture of the MSSM Higgs sector. In particular, more complete theoretical calculations are now available, and, more importantly, considerable work was done to obtain a systematic analysis of the MSSM Higgs sector. This includes a semi-automatic procedure to produce the contour curves in the MSSM parameter space and the analysis of channels not considered at the time of the TP.
\end{abstract}

CERN-TH/96-111

April 1996

${ }^{1}$ WWW address: http://hpjmiady.ifj.edu.pl/erichter/erichter.html 


\section{Contents}

1 Introduction $\quad 3$

2 Masses, couplings and widths 5

2.1 MSSM Higgs boson masses and couplings . . . . . . 5

2.2 Tree-level, one-loop and two-loop calculations . . . . . 7

2.3 Dependence of $m_{\mathrm{h}}$ and $m_{\mathrm{H}}$ on $m_{\mathrm{t}}$........... 10

2.4 Dependence of $\boldsymbol{m}_{\mathbf{h}}$ on the MSSM parameters . . . . . 14

2.5 The $h$ and $H$ couplings to fermions and gauge bosons . . 14

2.6 Higgs boson widths .............. 15

3 Branching ratios, cross-sections and rates 20

3.1 h-boson ................. 20

3.1.1 Branching ratios............ 20

3.1.2 Production cross-sections . . . . . . . . 23

3.1.3 Expected rates for signatures involving the h-boson 23

3.2 H-boson . . . . . . . . . . . . . . 31

3.2.1 Branching ratios ............. 31

3.2.2 Production cross-sections .......... 32

3.2.3 Expected rates for signatures involving the H-boson 42

3.3 A-boson ................. 47

3.3.1 Branching ratios ............. 47

3.3.2 Production cross-sections . . . . . . . 52

3.3.3 Expected rates for signatures involving the A-boson 53

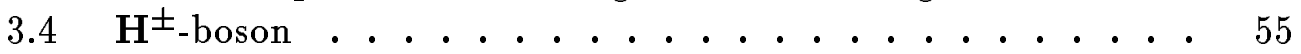

3.4.1 Branching ratios ............ 55

3.4.2 Production cross-sections .......... 56

3.4.3 Expected rates for signatures involving the $\mathbf{H}^{ \pm}$boson 56

4 Description of ATLAS simulation package 58

4.1 Interface to PYTHIA . . . . . . . . . . 58

4.2 MSSM branching ratios and cross-sections . . . . . . 59

4.3 Contour curves in the MSSM parameter space . . . . . 59

4.4 Default semi-automatic procedure . . . . . . . 61

5 Observability of MSSM Higgs bosons $\quad 64$

5.1 The $\mathbf{h} \rightarrow \gamma \gamma$ and $\mathbf{H} \rightarrow \gamma \gamma$ channels . . . . . . . 65

5.2 The $\mathbf{h} \rightarrow \mathbf{b} \overline{\mathbf{b}}$ channel ............. 83

5.3 The $\mathbf{H} \rightarrow \mathrm{ZZ}^{(\star)} \rightarrow 4 \ell$ channel .......... 87

5.4 The $\mathbf{H} / \mathbf{A} \rightarrow \tau \tau$ channel . . . . . . . . . . . 93

5.5 The $\mathbf{H} / \mathbf{A} \rightarrow \boldsymbol{\mu} \mu$ channel . . . . . . . . . . 100

5.6 The $\mathbf{H} \rightarrow \mathbf{h h}$ channel . . . . . . . . . . . 104

5.7 The $\mathbf{H} / \mathbf{A} \rightarrow \mathbf{t} \overline{\mathbf{t}}$ channel . . . . . . . . . . 110

5.8 The $\mathbf{A} \rightarrow \mathbf{Z h}$ channel ............ 116 
5.9 The $\mathrm{H}^{ \pm} \rightarrow \tau \nu$ channel . . . . . . . . . 122

6 Conclusions and outlook $\quad 126$

$\begin{array}{ll}\text { A Particle-level simulation } & 148\end{array}$

A.1 Jet reconstruction . . . . . . . . . . . . 148

A.2 Leptons and photons .............. 149

A.3 Parton-level versus particle-level ........... 149

A.4 b-tagging at the LHC . . . . . . . . . . 150

B The $\mathrm{H} / \mathrm{A} \rightarrow \mu \mu$ channel 151

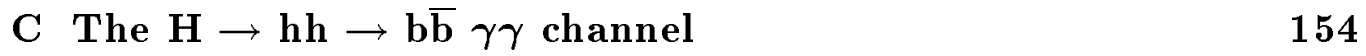

$\mathrm{D}$ The $\mathrm{H} / \mathrm{A} \rightarrow \mathrm{t} \overline{\mathrm{t}}$ channel $\quad 161$

E The $\mathrm{A} \rightarrow \mathrm{Zh} \rightarrow \ell \ell \mathrm{b} \overline{\mathrm{b}}$ channel $\quad 166$ 


\section{Introduction}

This report describes in detail the most recent evaluations of rates for signal and background processes in the Higgs sector of one of the possible extensions of the Standard Model (SM), the Minimal Supersymmetric Standard Model (MSSM). Such supersymmetric extensions of the SM require that the mass scale of the supersymmetric partners of ordinary particles be not significantly larger than the scale for electroweak symmetry breaking. The LHC has a crucial role to play in either uncovering or excluding such extensions to the SM.

To perform a systematic study of the Higgs sector of the MSSM one has to deal with a rich spectrum of possible signals. The Higgs sector contains two charged $\left(\mathrm{H}^{ \pm}\right)$and three neutral $(\mathrm{h}, \mathrm{H}, \mathrm{A})$ physical states. At the tree level, all Higgs boson masses and couplings can be expressed in terms of two parameters only, for example $m_{\mathrm{A}}$, the mass of the CP-odd boson, and $\tan \beta$, the ratio of the vacuum expectation values of the Higgs doublets. A complete study is therefore more complicated than in the SM, where the only free parameter in the Higgs sector is the Higgs mass. In addition, when considering production and decay of Higgs bosons, the whole particle spectrum of the model has to be taken into account, since the R-odd particles (squarks, sleptons, gauginos, higgsinos) can also play an important role. Nevertheless, the MSSM model has a high degree of predictivity. In particular, the radiative corrections to the masses and couplings of the Higgs bosons can be kept under control. The production cross-sections and decay branching ratios for the MSSM Higgs bosons can be readily computed in perturbation theory. The production or decay processes that correspond to the tree-level diagrams can be obtained from the corresponding formulae for the SM Higgs boson (e.g. see [1] for a summary) by simply multiplying the various amplitudes by the appropriate supersymmetric correction factors. For processes that are described by loop diagrams, however, one has to include in the MSSM model some contributions that are absent in the SM case.

Since the submission of the ATLAS TP [2], much has changed in the overall picture of the MSSM Higgs sector ${ }^{2}$. In particular, more complete theoretical calculations are now available, and, more importantly, considerable work was done in the analysis of the MSSM Higgs sector. This includes a more or less automatic procedure to produce the contour curves in the $\left(m_{\mathrm{A}}, \tan \beta\right)$ and $\left(m_{\mathrm{h}}, \tan \beta\right)$ planes and the analysis of channels not considered at the time of the TP (e.g. $\mathrm{H} \rightarrow \mathrm{hh}, \mathrm{H} / \mathrm{A} \rightarrow \mathrm{t} \overline{\mathrm{t}}$ and $\mathrm{A} \rightarrow \mathrm{Zh}$ ).

In the study presented here, two-loop calculations are used for the masses and couplings [4], as well as one-loop calculations [5] for some decay branching ratios $(\mathrm{H} \rightarrow \mathrm{hh})$. Following the approach of $[6], \mathrm{QCD}$ corrections are partially

\footnotetext{
${ }^{2}$ The analysis of the MSSM Higgs sector performed at the time of the TP is documented as a draft part of [3] in the ATLAS physics directory on WWW. This draft note can be considered as complementary in part to this one, since most of the figures for the branching ratios and cross-sections are presented there in a different way.
} 
taken into account by including running quark masses in the calculations of branching ratios, and it is assumed that all supersymmetric particles are heavy enough not to play an important role in the phenomenology of MSSM Higgs boson decays. In addition, negligible mixing in the stop and sbottom mass matrices is assumed, and the higgsino mass parameter $|\mu|$ is assumed to be negligible in comparison with the SUSY scale. This scenario corresponds to a fairly pessimistic discovery scenario for the LHC, since these choices for the additional MSSM parameters give the lowest possible upper limit for $m_{\mathrm{h}}$, which reduces the LHC potential for h-boson discovery in the $\mathrm{h} \rightarrow \gamma \gamma$ channel, but also suppresses the $\mathrm{h} \rightarrow \mathrm{ZZ}^{(\star)} \rightarrow 4 \ell$ channel.

The main uncertainty in the predictions for the MSSM Higgs sector arises from the present experimental uncertainty of $\sim \pm 15 \mathrm{GeV}[7]$ on the topquark mass $m_{\mathrm{t}}$. In contrast to the SM case, the MSSM Higgs sector is quite sensitive to the value of $m_{\mathrm{t}}$. For a consistent comparison with studies performed for LEP2 [8], a central value of $m_{\mathrm{t}}=175 \mathrm{GeV}$ is used throughout this note, and results are also shown for more extreme values of 150 and $200 \mathrm{GeV}$. Wherever relevant, results of the simulations performed for the SM Higgs sector and documented in [3] are used for comparison. If not explicitly stated otherwise, physics processes, including initial- and final-state radiation, hadronisation, and decays, were simulated using PYTHIA 5.7 [10] at $\sqrt{s}=14 \mathrm{TeV}$ and with its default set of structure function parametrisations. However, large uncertainties in the signal and background production cross-sections remain, due to higher-order corrections, structure function parametrisations, as well as the models used for full event generation. In addition, despite the existence of many higher-order QCD correction (Kfactor) calculations, not all processes of interest at the LHC (in particular background processes) have benefited from this theoretical effort. Therefore, the present studies have consistently and conservatively avoided the use of $\mathrm{K}$-factors, resorting to lowest-order predictions for both signals and backgrounds.

The results presented here come predominantly from particle-level simulations. However, most of the crucial detector-dependent performance figures, such as the mass resolutions, reconstruction/identification efficiencies and background rejections, were obtained using full simulations of the ATLAS detector, and used as inputs to the analysis of signal and background rates presented here.

The systematic studies presented in this report allow definite conclusions to be drawn concerning the ATLAS discovery potential over the whole $\left(m_{\mathrm{A}}, \tan \beta\right)$ and $\left(m_{\mathrm{h}}, \tan \beta\right)$ parameter space. They also cover a rich spectrum of experimental signatures, thus providing a good benchmark to study the flexibility and robustness of the ATLAS detector to discover new physics. It should be nevertheless stressed that the present results should be treated with some caution. Theoretical and experimental uncertainties may change the positions of discovery curves in a significant way; the dominant theoretical ones are: 
- the top-quark mass $m_{\mathrm{t}}$,

- the MSSM parameters,

- the mass spectrum of SUSY particles,

- the K-factor corrections to signal and background production,

- the structure function parametrisations,

- the higher-order radiative corrections to the MSSM Higgs boson masses and couplings.

This note is organised as follows: Sections 2 and 3 quantify the general properties of the MSSM model; in particular, the relations between the various Higgs boson masses, couplings, widths, production cross-sections and branching ratios are discussed as well as the expected rates at the LHC for the interesting channels. Special emphasis is given to the variations of the predictions as a function of $m_{\mathrm{t}}$ and the MSSM parameters, $m_{\mathrm{A}}$ and $\tan \beta$. For decay channels accessible in the SM Higgs sector, SM predictions are also shown, in order to shed light on the differences or similarities between the two models. Section 4 describes the semi-automatic procedure used to produce the $5 \sigma$-discovery contour curves in the MSSM parameter space. Section 5 discusses the observability of different decay channels, summarising in a systematic way the signal and background rates as well as the expected significances at low and high luminosities. Numbers are quoted for a few mass values for each decay channel. The $5 \sigma$-discovery contour curves are presented for each decay channel in the $\left(m_{\mathrm{A}}, \tan \beta\right)$ and $\left(m_{\mathrm{h}}, \tan \beta\right)$ planes, for the three values of $m_{\mathrm{t}}$ given above and for three values of the integrated luminosity. Section 6 summarises the results of this study, showing the global $5 \sigma$-discovery contour curves, and also drawing some conclusions on the comparison with LEP2, and on the ATLAS potential to disentangle between SM and MSSM. For completeness, global $5 \sigma$-discovery contour curves are also shown for the combined ATLAS and CMS detectors. In Appendices A to E, the simulation framework and the recent work on channels not studied at the time of the TP are described in more detail.

\section{Masses, couplings and widths}

\subsection{MSSM Higgs boson masses and couplings}

In the MSSM, the Higgs sector [1] contains two charged physical states $\left(\mathrm{H}^{ \pm}\right)$ and three neutral ones (h, H, A). At the tree level, all Higgs boson masses and couplings can be expressed in terms of two parameters only, for example $m_{A}$ and $\tan \beta$. The following relations can be used:

$$
\begin{aligned}
& m_{H, h}^{2}=\frac{1}{2}\left[m_{A}^{2}+m_{Z}^{2} \pm \sqrt{\left(m_{A}^{2}+m_{Z}^{2}\right)^{2}-4 m_{A}^{2} m_{Z}^{2} \cos ^{2} 2 \beta}\right] \\
& m_{H^{ \pm}}^{2}=m_{W}^{2}+m_{A}^{2}
\end{aligned}
$$

The mixing angle $\alpha\left(-\frac{\pi}{2}<\alpha<0\right)$ required to diagonalise the Higgs mass 
matrix is given by the following expression:

$$
\cos 2 \alpha=-\cos 2 \beta \frac{m_{A}^{2}-m_{Z}^{2}}{m_{H}^{2}-m_{h}^{2}} .
$$

The couplings for the neutral Higgs bosons to fermions and massive gauge bosons are easily obtained ${ }^{3}$ from the SM Higgs couplings, shown in Table 1, if one multiplies them by the $\alpha$ - and $\beta$-dependent factors summarised in Table 2.

Table 1: SM Higgs couplings to fermions and massive gauge bosons.

\begin{tabular}{|c||c|c|c|}
\hline SM & All fermions & WW & ZZ \\
\hline \hline $\mathrm{H}$ & $-\frac{i g m_{f}}{2 m_{W}}$ & $i g m_{W} g^{\mu \nu}$ & $\frac{i g m_{Z}}{2 \cos \theta_{W}} g^{\mu \nu}$ \\
\hline
\end{tabular}

Table 2: MSSM correction factors to Higgs boson couplings with respect to the $S M$ couplings to fermions and massive gauge bosons.

\begin{tabular}{|c||c|c|c|}
\hline MSSM & $\begin{array}{c}\mathrm{d} \overline{\mathrm{d}}, \mathrm{s} \overline{\mathrm{s}}, \mathrm{b} \overline{\mathrm{b}} \\
\mathrm{e}^{+} \mathrm{e}^{-}, \mu^{+} \mu^{-}, \tau^{+} \tau^{-}\end{array}$ & $\mathrm{u} \overline{\mathrm{u}}, \mathrm{c} \overline{\mathrm{c}}, \mathrm{t} \overline{\mathrm{t}}$ & $\mathrm{WW}, \mathrm{ZZ}$ \\
\hline \hline $\mathrm{h}$ & $-\sin \alpha / \cos \beta$ & $\cos \alpha / \sin \beta$ & $\sin (\beta-\alpha)$ \\
\hline $\mathrm{H}$ & $\cos \alpha / \cos \beta$ & $\sin \alpha / \sin \beta$ & $\cos (\beta-\alpha)$ \\
\hline $\mathrm{A}$ & $-\mathrm{i} \gamma_{5} \tan \beta$ & $-\mathrm{i} \gamma_{5} \cot \beta$ & 0 \\
\hline
\end{tabular}

These tree-level formulae for the Higgs-boson masses and couplings are however subject to large radiative corrections, dominated by the exchange of virtual top and bottom quarks and squarks in the loop diagrams. They introduce a dependence on the top and squark masses and on the mixing in the stop-sbottom mass matrices into the formulae for the Higgs masses and couplings (see e.g. [5]). In particular, the largest possible value of $m_{\mathrm{h}}$ is significantly larger than $m_{\mathrm{Z}}$ for $m_{\mathrm{t}}>150 \mathrm{GeV}$.

When considering MSSM Higgs boson production and decay, the most important part of these radiative corrections is taken into account by using corrected formulae for the mass matrix and then determining $\alpha$ from the input parameters. Couplings to fermions/gauge bosons can still be expressed in terms of the fermion/gauge boson masses and of the angles $\beta$ and $\alpha$. The leading radiative corrections can thus be taken into account by using the corrected expression for $\alpha$ and the running fermion masses, evaluated at the

\footnotetext{
${ }^{3}$ The complete set of Feynman rules for the MSSM Higgs sector can be found in [1].
} 
scale $Q$ which characterises the process under consideration. When making numerical calculations, this note always uses a universal soft supersymmetrybreaking squark mass $M_{S U S Y}=m_{Q}=m_{U}=m_{D}=m_{\bar{q}}=1 \mathrm{TeV}$, always assumes that all SUSY particle masses are at $1 \mathrm{TeV}$, that the mixing in the stop and sbottom mass matrices is negligible, i.e. that: $A_{t}=A_{b}=0$, and that the value of the Higgsino-mass term $|\mu|$ is much smaller than $M_{S U S Y}$.

\subsection{Tree-level, one-loop and two-loop calculations}

Over the last few years, different methods for computing the radiative corrections to the Higgs mass spectrum have been developed, see e.g. [8] for more details. The effect of these radiative corrections is significant for the light Higgs mass $m_{\mathrm{h}}$, shifting upwards its maximum value by as much as $\sim 50 \mathrm{GeV}$ with respect to the tree-level predictions. This effect is even larger when including only one-loop radiative corrections, but is reduced if the dominant terms of the two-loop corrections are taken into account. In the following, results from calculations including one-loop radiative corrections in the Effective Potential Approach [5], as given by the code of [6], are compared to to those including a Renormalisation Group improvement of the Effective Potential at one loop as well as the dominant two-loop effects [4].

Fig. 1 shows $m_{\mathrm{h}}$ as a function of $m_{\mathrm{A}}$ for tree-level (dots), one-loop (dashed line) and two-loop (solid line) calculations, for different values of $\tan \beta$ and for $m_{\mathrm{t}}=175 \mathrm{GeV}$. The value of $m_{\mathrm{h}}$ depends very little on $m_{\mathrm{A}}$ for $m_{\mathrm{A}}>200 \mathrm{GeV}$ and reaches its maximum allowed value for $m_{\mathrm{A}}>200 \mathrm{GeV}$ and $\tan \beta>10$. The effect of the radiative corrections is significant, leading to an increase of this maximum allowed value of $30-40 \mathrm{GeV}$ (for $m_{\mathrm{t}}=175 \mathrm{GeV}$ ) with respect to the tree-level predictions. The one-loop calculations [5] used at the time of the TP overestimate, for the same set of MSSM parameters, the maximum allowed value of $m_{\mathrm{h}}$ by about $10 \mathrm{GeV}$ with respect to the more recent two-loop calculations [4].

In the case of $m_{\mathrm{H}}$, the dependence on $m_{\mathrm{A}}$ is nearly linear, as shown in Fig. 2, with $m_{\mathrm{H}} \sim m_{\mathrm{A}}$ for large $m_{\mathrm{A}}$ and the effect of radiative corrections is noticeable only for small $m_{\mathrm{A}}\left(m_{\mathrm{A}}<200 \mathrm{GeV}\right)$, leading to an increase of $m_{\mathrm{H}}$ of $10-20 \%$. If $\tan \beta$ increases from 3 to 30 , the minimum allowed value of $m_{\mathrm{H}}$ decreases by about $10 \mathrm{GeV}$, from $m_{\mathrm{H}}=123 \mathrm{GeV}$ to $m_{\mathrm{H}}=113 \mathrm{GeV}$, and is

reached for $m_{\mathrm{A}}<110 \mathrm{GeV}$ and $\tan \beta>10$. For the charged Higgs $\mathrm{H}^{ \pm}$the effect of the radiative corrections is small and $m_{\mathrm{H}^{ \pm}}$increases only slightly with $\tan \beta$, by $5-10 \mathrm{GeV}$ when $\tan \beta$ increases from 3 to 30 .

The dependence of the coupling $\cos 2 \alpha$ on $m_{\mathrm{A}}$ is shown in Fig. 3 for different values of $\tan \beta$. For large values of $m_{\mathrm{A}}$ and $\tan \beta, \cos 2 \alpha$ is very close to unity and does not depend on the radiative corrections. For smaller values of $\tan \beta$ or $m_{\mathrm{A}}$ the two-loop predictions lie between the tree-level and one-loop ones ( similarly to $m_{\mathrm{h}}$ ). The numerical values of the correction factors to the $\mathrm{h}$ and H-boson couplings shown in Table 2 have to be changed correspondingly, as explained above. 


\section{h-boson mass}
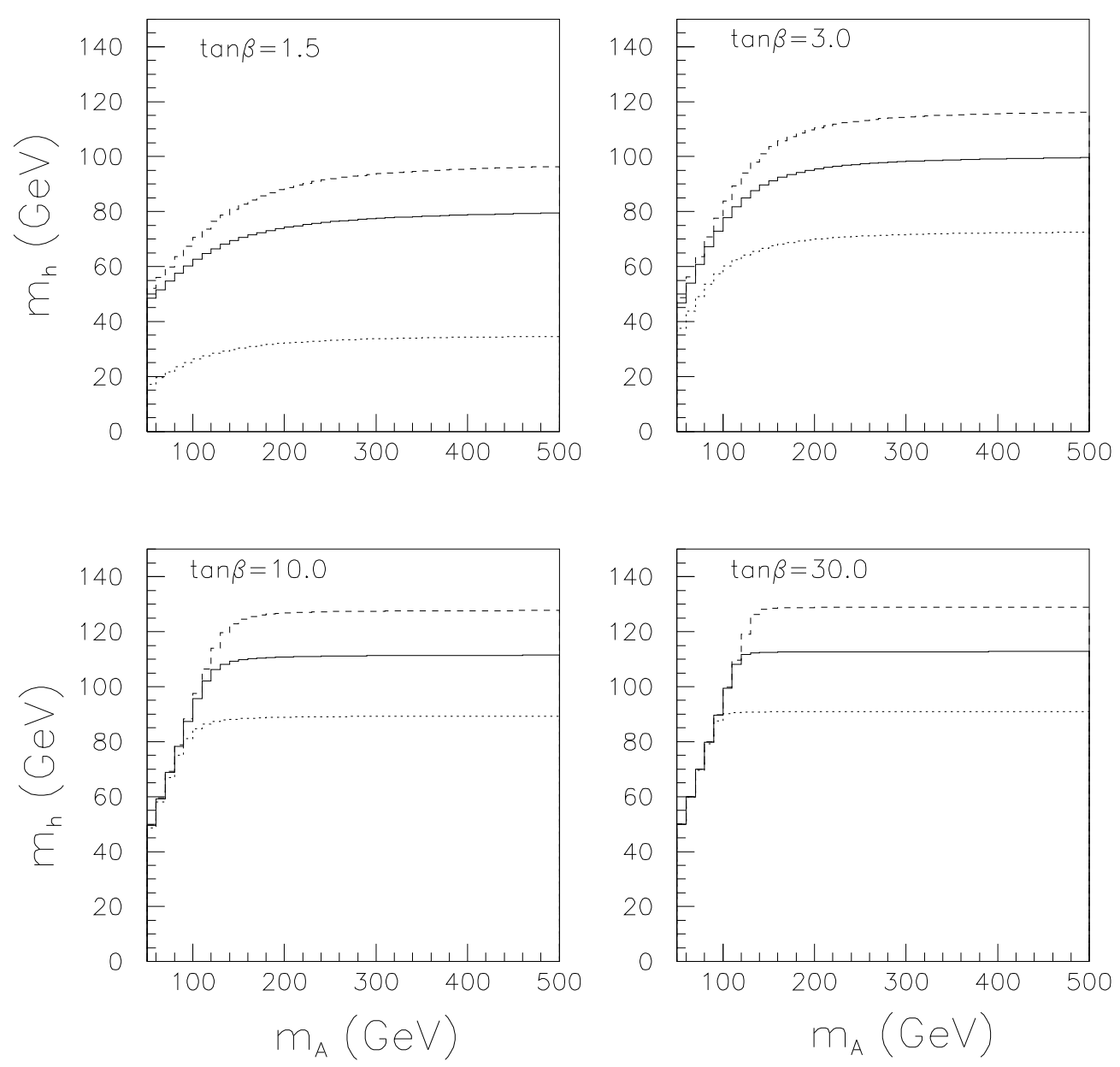

Figure 1: Tree-level (dots), one-loop (dashed) and two-loop (solid) predictions for $m_{\mathrm{h}}$ as a function of $m_{\mathrm{A}}$ for four values of $\tan \beta$ and for $m_{\mathrm{t}}=175 \mathrm{GeV}$. 
$H-b o s o n$ mass
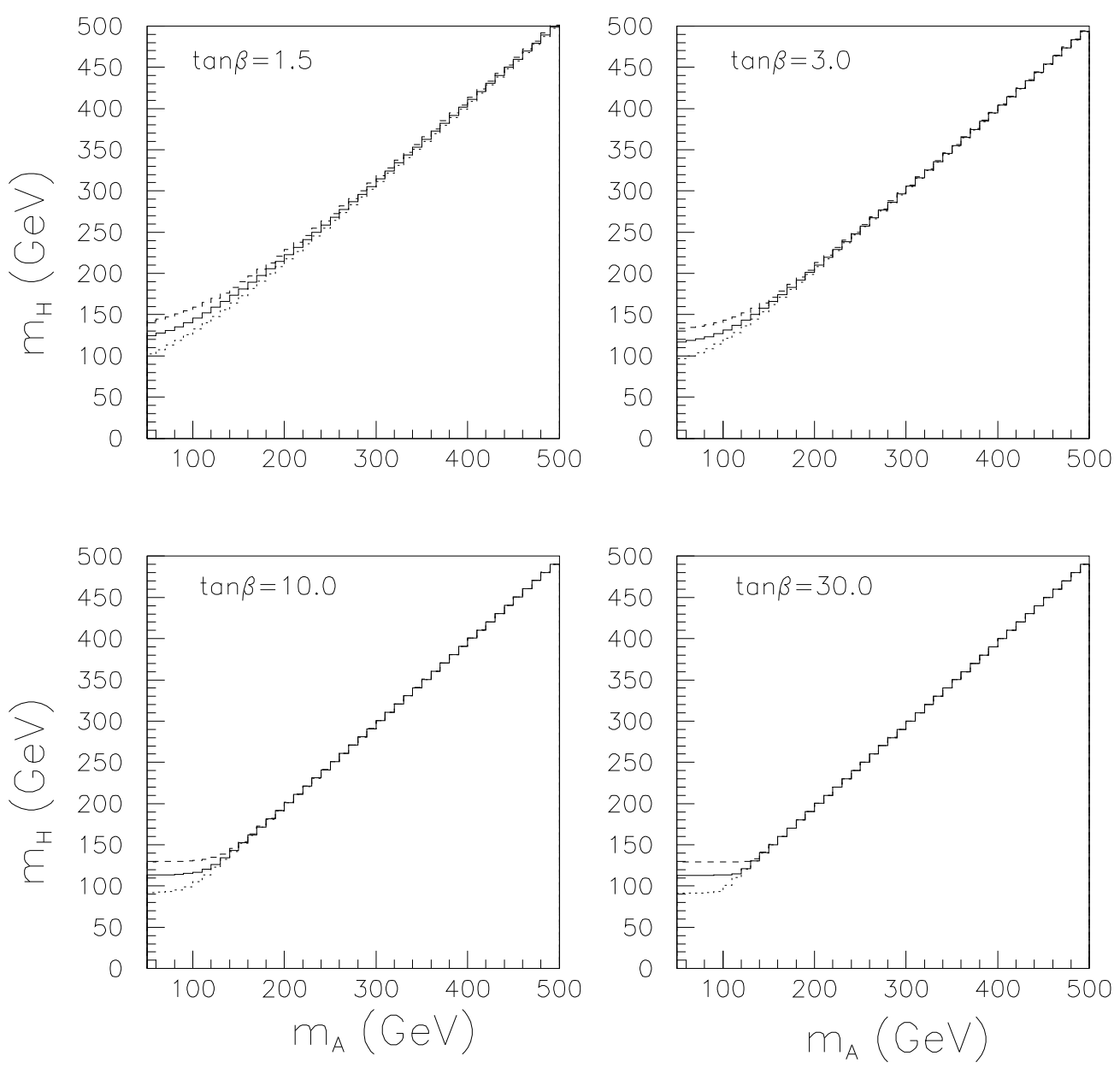

Figure 2: Tree-level (dots), one-loop (dashed) and two-loop (solid) predictions for $m_{\mathrm{H}}$ as a function of $m_{\mathrm{A}}$ for four values of $\tan \beta$ and for $m_{\mathrm{t}}=175 \mathrm{GeV}$. 

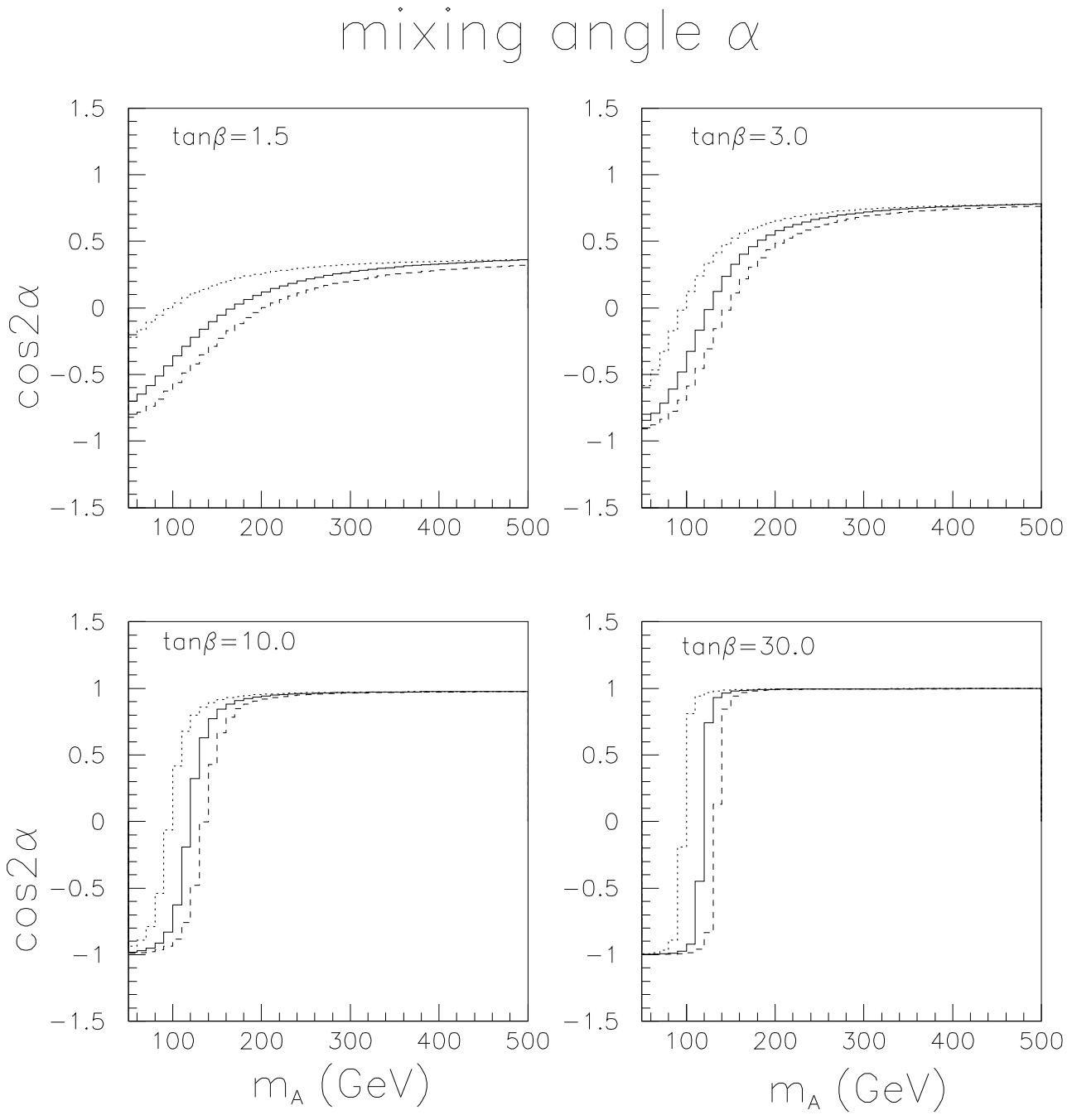

Figure 3: Tree-level (dots), one-loop (dashed) and two-loop (solid) predictions for the mixing angle $\alpha$ as a function of $m_{\mathrm{A}}$, for four values of $\tan \beta$ and for $m_{\mathrm{t}}=175 \mathrm{GeV}$.

\section{$2.3 \quad$ Dependence of $m_{\mathrm{h}}$ and $m_{\mathrm{H}}$ on $m_{\mathrm{t}}$}

Another consequence of these radiative corrections is that the expressions for $m_{\mathrm{h}}$ and $m_{\mathrm{H}}$ now depend on $m_{\mathrm{t}}$. In particular, the maximum allowed value of $m_{\mathrm{h}}$ is shifted upwards by a factor proportional to the fourth power of $m_{\mathrm{t}}$. This explains why the tree-level predictions for $m_{\mathrm{h}}$ are considerably affected by the radiative corrections for large values of $m_{\mathrm{t}}$. For $m_{\mathrm{t}}=150,175$ and $200 \mathrm{GeV}$ and $\tan \beta=3$ and 30 , the two-loop numerical values of the MSSM Higgs-boson masses are given as a function of $m_{\mathrm{A}}$ in Tables 3 and 4 for $m_{\mathrm{h}}$, Tables 5 and 6 for $m_{\mathrm{H}}$ and Tables 7 and 8 for $m_{\mathrm{H}^{ \pm}}$. 
Table 3: For $\tan \beta=3$ and $m_{\mathrm{t}}=150,175$, and $200 \mathrm{GeV}$, dependence of $m_{\mathrm{h}}$ on $m_{\mathrm{A}}$.

\begin{tabular}{|c||c|c|c|}
\hline$m_{\mathrm{A}}(\mathrm{GeV})$ & $m_{\mathrm{t}}=150 \mathrm{GeV}$ & $m_{\mathrm{t}}=175 \mathrm{GeV}$ & $m_{\mathrm{t}}=200 \mathrm{GeV}$ \\
\hline \hline 80.0 & 62.4 & 67.1 & 71.3 \\
100.0 & 71.1 & 77.7 & 84.1 \\
120.0 & 76.6 & 85.0 & 94.0 \\
140.0 & 80.0 & 89.6 & 100.7 \\
160.0 & 82.1 & 92.4 & 105.0 \\
180.0 & 83.5 & 94.3 & 107.7 \\
200.0 & 84.5 & 95.5 & 109.4 \\
240.0 & 85.7 & 97.1 & 111.6 \\
280.0 & 86.4 & 98.0 & 112.8 \\
320.0 & 86.9 & 98.7 & 113.6 \\
360.0 & 87.2 & 98.9 & 114.1 \\
400.0 & 87.4 & 99.2 & 114.4 \\
500.0 & 87.8 & 99.6 & 115.0 \\
\hline
\end{tabular}

Table 4: For $\tan \beta=30$ and $m_{\mathrm{t}}=150,175$, and $200 \mathrm{GeV}$, dependence of $m_{\mathrm{h}}$ on $m_{\mathrm{A}}$.

\begin{tabular}{|c||c|c|c|}
\hline$m_{A}(\mathrm{GeV})$ & $m_{t}=150 \mathrm{GeV}$ & $m_{t}=175 \mathrm{GeV}$ & $m_{t}=200 \mathrm{GeV}$ \\
\hline \hline 80.0 & 79.7 & 79.8 & 79.9 \\
100.0 & 98.1 & 99.4 & 99.7 \\
120.0 & 102.2 & 111.7 & 118.8 \\
140.0 & 102.4 & 112.5 & 125.8 \\
160.0 & 102.5 & 112.6 & 126.2 \\
180.0 & 102.5 & 112.7 & 126.3 \\
200.0 & 102.6 & 112.7 & 126.4 \\
240.0 & 102.6 & 112.7 & 126.4 \\
280.0 & 102.6 & 112.7 & 126.4 \\
320.0 & 102.6 & 112.7 & 126.4 \\
360.0 & 102.6 & 112.8 & 126.4 \\
400.0 & 102.6 & 112.8 & 126.5 \\
500.0 & 102.6 & 112.8 & 126.5 \\
\hline
\end{tabular}


Table 5: For $\tan \beta=3$ and $m_{\mathrm{t}}=150,175$, and $200 \mathrm{GeV}$, dependence of $m_{\mathrm{H}}$ on $m_{\mathrm{A}}$.

\begin{tabular}{|c||c|c|c|}
\hline$m_{\mathrm{A}}(\mathrm{GeV})$ & $m_{\mathrm{t}}=150 \mathrm{GeV}$ & $m_{\mathrm{t}}=175 \mathrm{GeV}$ & $m_{\mathrm{t}}=200 \mathrm{GeV}$ \\
\hline \hline 80.0 & 115.8 & 123.5 & 135.5 \\
100.0 & 125.9 & 131.6 & 141.3 \\
120.0 & 139.4 & 143.3 & 150.3 \\
140.0 & 155.2 & 157.9 & 162.8 \\
160.0 & 172.5 & 174.4 & 177.8 \\
180.0 & 190.6 & 192.0 & 194.5 \\
200.0 & 209.2 & 210.3 & 212.3 \\
240.0 & 247.3 & 248.0 & 249.3 \\
280.0 & 286.0 & 286.6 & 287.5 \\
320.0 & 325.2 & 325.6 & 326.4 \\
360.0 & 364.5 & 364.9 & 365.5 \\
400.0 & 404.0 & 404.3 & 404.8 \\
500.0 & 503.2 & 503.4 & 503.8 \\
\hline
\end{tabular}

Table 6: For $\tan \beta=30$ and $m_{\mathrm{t}}=150,175$, and $200 \mathrm{GeV}$, dependence of $m_{\mathrm{H}}$ on $m_{\mathrm{A}}$.

\begin{tabular}{|c||c|c|c|}
\hline$m_{\mathrm{A}}(\mathrm{GeV})$ & $m_{\mathrm{t}}=150 \mathrm{GeV}$ & $m_{\mathrm{t}}=175 \mathrm{GeV}$ & $m_{\mathrm{t}}=200 \mathrm{GeV}$ \\
\hline \hline 80.0 & 103.0 & 113.1 & 126.7 \\
100.0 & 104.6 & 113.5 & 126.9 \\
120.0 & 120.5 & 121.1 & 127.7 \\
140.0 & 140.3 & 140.4 & 140.7 \\
160.0 & 160.2 & 160.2 & 160.3 \\
180.0 & 180.1 & 180.2 & 180.2 \\
200.0 & 200.1 & 200.1 & 200.2 \\
240.0 & 240.1 & 240.1 & 240.1 \\
280.0 & 280.1 & 280.1 & 280.1 \\
320.0 & 320.1 & 320.1 & 320.1 \\
360.0 & 360.1 & 360.1 & 360.1 \\
400.0 & 400.0 & 400.0 & 400.0 \\
500.0 & 500.0 & 500.0 & 500.0 \\
\hline
\end{tabular}


Table 7: For $\tan \beta=3$ and $m_{\mathrm{t}}=150,175$, and $200 \mathrm{GeV}$, dependence of $m_{\mathrm{H}^{ \pm}}$ on $m_{\mathrm{A}}$.

\begin{tabular}{|c||c|c|c|}
\hline$m_{A}(\mathrm{GeV})$ & $m_{\mathrm{t}}=150 \mathrm{GeV}$ & $m_{\mathrm{t}}=175 \mathrm{GeV}$ & $m_{\mathrm{t}}=200 \mathrm{GeV}$ \\
\hline \hline 60.0 & 98.8 & 97.9 & 97.4 \\
70.0 & 105.1 & 104.3 & 103.9 \\
80.0 & 112.0 & 111.3 & 110.9 \\
90.0 & 119.4 & 118.7 & 118.3 \\
100.0 & 127.1 & 126.4 & 126.1 \\
110.0 & 135.1 & 134.5 & 134.1 \\
120.0 & 143.4 & 142.8 & 142.5 \\
130.0 & 151.8 & 151.3 & 151.0 \\
140.0 & 160.5 & 160.0 & 159.7 \\
150.0 & 169.0 & 168.8 & 168.5 \\
160.0 & 177.9 & 177.7 & 177.5 \\
170.0 & 187.0 & 186.8 & 186.5 \\
180.0 & 196.1 & 195.9 & 195.7 \\
\hline
\end{tabular}

Table 8: For $\tan \beta=30$ and $m_{\mathrm{t}}=150,175$, and $200 \mathrm{GeV}$, dependence of $m_{\mathrm{H}^{ \pm}}$on $m_{\mathrm{A}}$.

\begin{tabular}{|c||c|c|c|}
\hline$m_{\mathrm{A}}(\mathrm{GeV})$ & $m_{\mathrm{t}}=150 \mathrm{GeV}$ & $m_{\mathrm{t}}=175 \mathrm{GeV}$ & $m_{\mathrm{t}}=200 \mathrm{GeV}$ \\
\hline \hline 60.0 & 100.9 & 101.4 & 102.0 \\
70.0 & 107.1 & 107.6 & 108.2 \\
80.0 & 113.9 & 114.4 & 114.9 \\
90.0 & 121.1 & 121.6 & 122.1 \\
100.0 & 128.7 & 129.2 & 129.7 \\
110.0 & 136.7 & 137.1 & 137.5 \\
120.0 & 144.8 & 145.2 & 145.6 \\
130.0 & 153.2 & 153.6 & 154.0 \\
140.0 & 161.8 & 162.1 & 162.5 \\
150.0 & 170.5 & 170.8 & 171.2 \\
160.0 & 179.4 & 179.7 & 180.0 \\
170.0 & 188.4 & 188.6 & 189.0 \\
180.0 & 197.4 & 197.7 & 198.0 \\
\hline
\end{tabular}


When $m_{\mathrm{t}}$ increases from 150 to $200 \mathrm{GeV}$, the maximum allowed value of $m_{\mathrm{h}}$ increases by $25 \mathrm{GeV}$. On the other hand, the dependence of $m_{\mathrm{H}}$ on $m_{\mathrm{t}}$ is very weak and limited to small values of $m_{\mathrm{A}}\left(m_{\mathrm{A}}<200 \mathrm{GeV}\right)$. The charged Higgs mass depends very weakly on both $\tan \beta$ and $m_{\mathrm{t}}$, and changes by less than $1 \mathrm{GeV}$ when $m_{\mathrm{t}}$ increases from 150 to $200 \mathrm{GeV}$.

\subsection{Dependence of $m_{\mathrm{h}}$ on the MSSM parameters}

The dependence of the maximum allowed value of $m_{\mathrm{h}}$ on the other MSSM parameters has been recently discussed in [8]. For the usual choice of SUSY scale $M_{S U S Y}=1 \mathrm{TeV}$, a few choices for the values of the higgsino mass parameter $\mu$ and the soft SUSY breaking parameter, $A_{t}=A_{b}=A$, have been studied. In particular, it has been shown that the maximum allowed value of $m_{\mathrm{h}}$, for scenarios with negligible mixing, $A \sim|\mu| \ll M_{S U S Y}$, is lower than for scenarios with maximal mixing, $A=\sqrt{6} M_{S U S Y},|\mu| \ll M_{S U S Y}$, by about $30 \mathrm{GeV}$.

This difference is very similar in magnitude to the one quoted above, if $m_{\mathrm{t}}$ varies from 150 to $200 \mathrm{GeV}$. This reprot will therefore be limited to a study of the impact of variations of $m_{\mathrm{t}}$ on the expected sensitivity to the MSSM Higgs sector, and this impact will be considered as typical of what one might expect for variations of $\mu$ and $A$. More complete theoretical calculations are now available for studying directly, the dependence, not only upon $\mu$ and $A$, but also upon $M_{S U S Y}$ and more generally upon the SUSY particle mass spectrum (see the conclusions for a further discussion of the interplay between the MSSM Higgs sector and the supersymmetric particle sector).

\subsection{The $h$ and $H$ couplings to fermions and gauge bosons}

Fig. 4 presents the correction factors to the MSSM h-boson couplings relative to the SM couplings to fermions and massive gauge bosons (see Table 2) as a function of $m_{\mathrm{A}}$ (left side) and of $m_{\mathrm{h}}$ (right side), for $m_{\mathrm{t}}=175 \mathrm{GeV}$ and for different values of $\tan \beta$. For large values of $\tan \beta$, the couplings to down-type quarks and leptons are strongly enhanced, while those to up-type quarks and massive gauge bosons are suppressed. All correction factors tend towards unity for large $m_{A}$, but this effect is slower in the case of the enhanced couplings to down-type quarks and leptons than in the case of the suppressed couplings. For large values of $m_{\mathrm{A}}$ and $\tan \beta$, the h-boson couplings therefore tend towards the SM Higgs couplings. The plateau observed over a large range of $m_{\mathrm{A}}$ values on the left side of Fig. 4 is mapped onto a very small interval of $m_{\mathrm{h}}$ values on the right side of Fig. 4 . This will most often result in a very steep behaviour of h-boson production and decay rates for values of $m_{\mathrm{h}}$ near the maximum allowed value.

Fig. 5 presents the same correction factors for the $\mathrm{H}$-boson couplings. In contrast to the case of the h-boson, the couplings of the MSSM H-boson 
to down-type quarks and leptons are strongly enhanced with respect to the SM couplings over a large region of the parameter space $(\tan \beta>10$ and $\left.m_{\mathrm{A}}>100 \mathrm{GeV}\right)$. Over this region, the couplings of the H-boson to up-type quarks are strongly suppressed; those to massive gauge bosons are even more strongly suppressed, for all values of $\tan \beta$ and for almost all values of $m_{\mathrm{A}}$ $\left(m_{\mathrm{A}}>120 \mathrm{GeV}\right)$.

\subsection{Higgs boson widths}

The total decay widths of the MSSM Higgs bosons all differ significantly from that of a SM Higgs boson of the same mass. Tables 9 to 12 show how these widths vary as a function of mass and of $\tan \beta$, for $m_{\mathrm{t}}=175 \mathrm{GeV}$ and for $\mathrm{h}, \mathrm{H}, \mathrm{A}$ and $\mathrm{H}^{ \pm}$respectively. For comparison, the corresponding width of a SM Higgs boson of the same mass is also given.

The decay width of the h-boson is usually larger than that of a SM Higgs boson of the same mass, and increases significantly with $\tan \beta$, up to $\sim 5 \mathrm{GeV}$ for $\tan \beta=50$. However, even for large values of $\tan \beta$, the h-boson decay width tends towards the SM Higgs boson width, as the h-boson mass tends towards its maximum allowed value. As shown in Table 4, the region where $m_{\mathrm{h}}$ is close to its maximum allowed value maps onto most of the relevant parameter space in the $\left(m_{\mathrm{A}}, \tan \beta\right)$ plane. As a consequence, the h-boson width will always be much smaller than the experimental resolutions for the signatures accessible at the LHC.

The decay widths of the H-boson and A-boson are very similar for $m_{\mathrm{H}}=m_{\mathrm{A}}$. They become large, 3 to $25 \mathrm{GeV}$, for large values of $m_{\mathrm{H}}, m_{\mathrm{A}}$ and of $\tan \beta$, and will have to be taken into account, when studying the channels accessible in this region of parameter space $(\mathrm{H} / \mathrm{A} \rightarrow \tau \tau$ and $\mu \mu)$. For small values

of $\tan \beta$, where the channel $\mathrm{H} \rightarrow \mathrm{ZZ}^{(*)} \rightarrow 4 \ell$ is accessible for $m_{\mathrm{H}}<2 m_{\mathrm{t}}$, the $\mathrm{H}$-boson width is much smaller than that of the SM Higgs boson of the same mass.

Finally, Table 12 shows that the decay width of the charged Higgs boson is small, less than $\sim 1 \mathrm{GeV}$, for all values of $\tan \beta$ and for $m_{\mathrm{H}^{ \pm}}<m_{\mathrm{t}}$. 


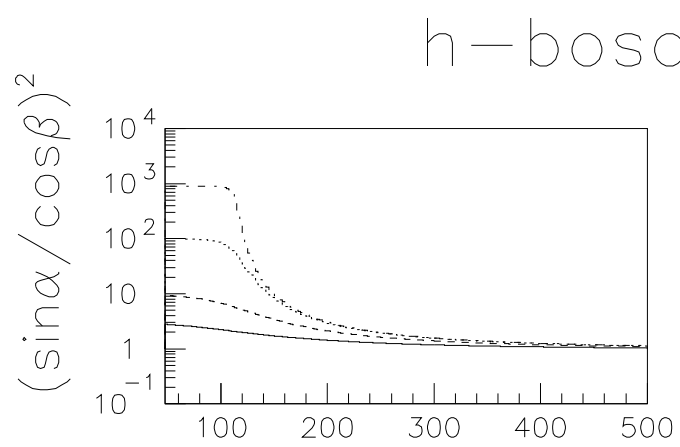

couplings
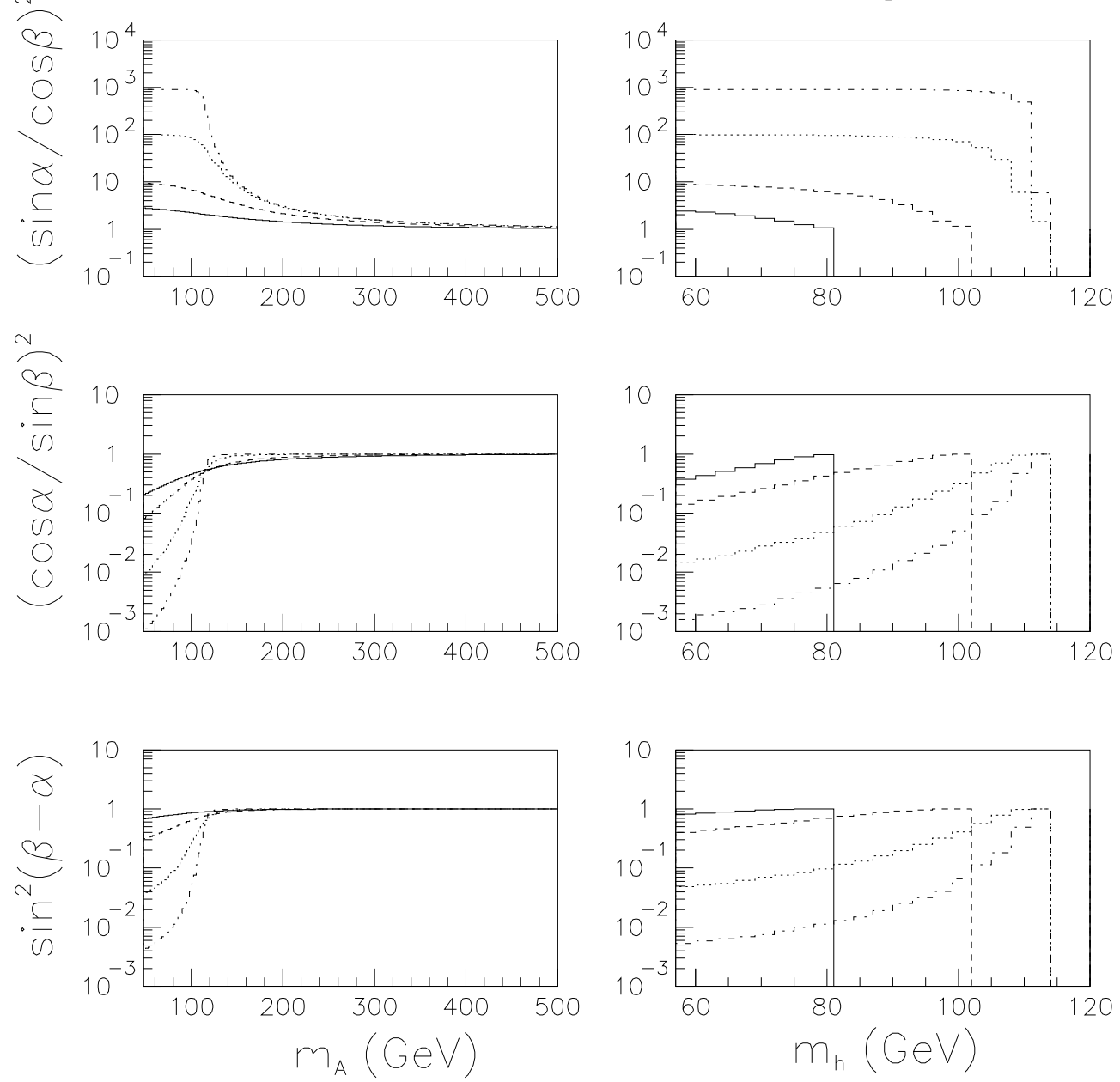

Figure 4: Two-loop predictions for the MSSM correction factors to the $h$ boson couplings to fermions and massive gauge bosons as a function of $m_{\mathrm{A}}$ (left side) and of $m_{\mathrm{h}}$ (right side) for $m_{\mathrm{t}}=175 \mathrm{GeV}$ and for different values of $\tan \beta$. The solid lines are for $\tan \beta=1.5$, the dashed lines for $\tan \beta=3.0$, the dotted lines for $\tan \beta=10.0$ and the dot-dashed lines for $\tan \beta=30.0$. 

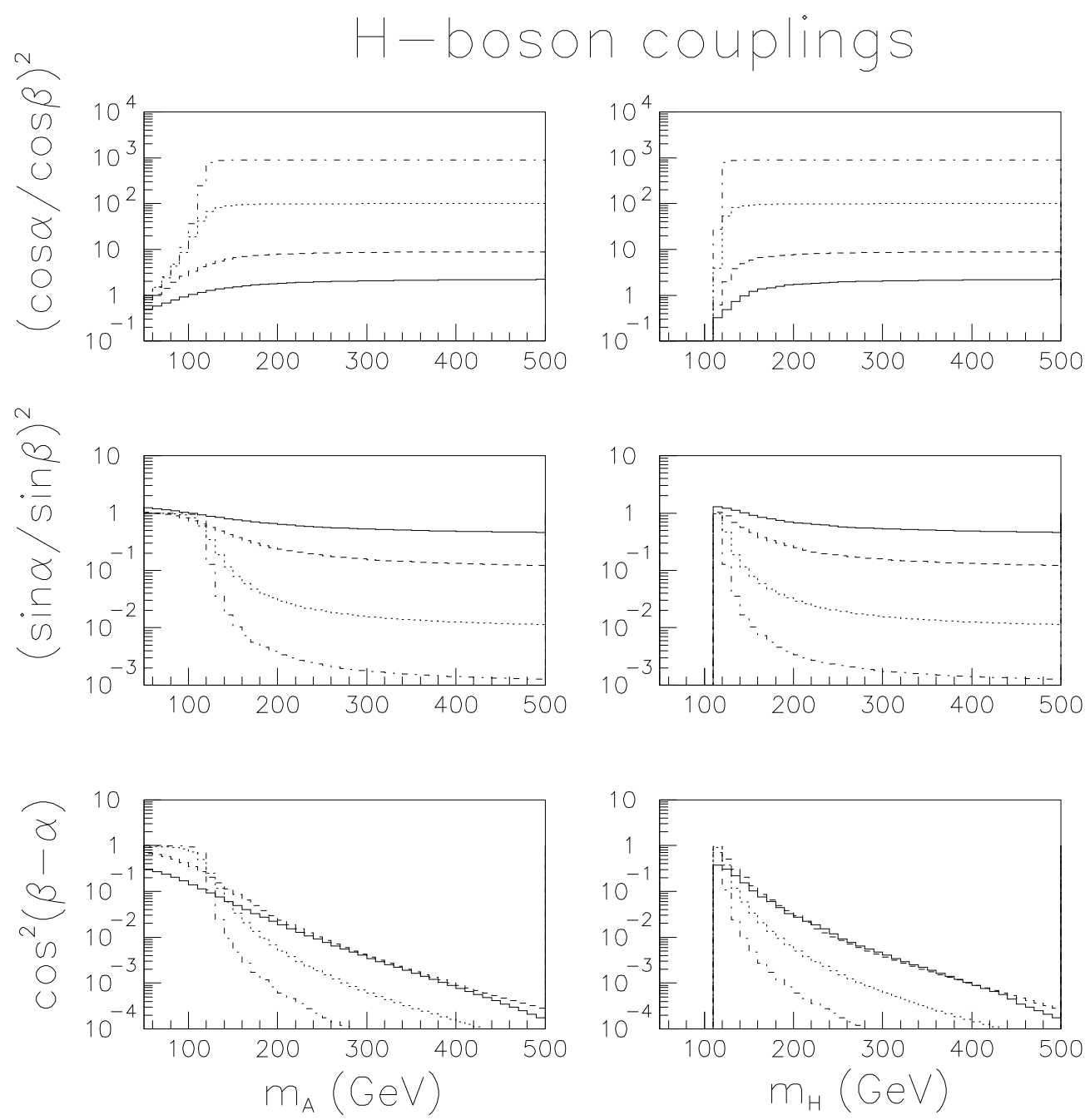

Figure 5: The two-loop predictions for the MSSM correction factors to the $H$-boson couplings to fermions and massive gauge bosons as a function of $m_{\mathrm{A}}$ (left side) and of $m_{\mathrm{H}}$ (right side) for $m_{\mathrm{t}}=175 \mathrm{GeV}$ and for different values of $\tan \beta$. The solid lines are for $\tan \beta=1.5$, the dashed lines for $\tan \beta=3.0$, the dotted lines for $\tan \beta=10.0$ and the dot-dashed lines for $\tan \beta=30.0$. 
Table 9: Total decay width of the h-boson for $m_{\mathrm{t}}=175 \mathrm{GeV}$.

\begin{tabular}{|c|c|c|c|c|c|}
\hline$m_{\mathrm{h}}(\mathrm{GeV})$ & $\begin{array}{l}\Gamma^{\mathrm{tot}}(\mathrm{GeV}) \\
\text { SM Higgs }\end{array}$ & $\begin{array}{c}\Gamma_{\mathrm{h}}^{\operatorname{tot}}(\mathrm{GeV}) \\
\tan \beta=3.0\end{array}$ & $\begin{array}{c}\Gamma_{\mathrm{h}}^{\mathrm{tot}}(\mathrm{GeV}) \\
\tan \beta=10.0\end{array}$ & $\begin{array}{c}\Gamma_{\mathrm{h}}^{\mathrm{tot}}(\mathrm{GeV}) \\
\tan \beta=30.0\end{array}$ & $\begin{array}{c}\Gamma_{\mathrm{h}}^{\mathrm{tot}}(\mathrm{GeV}) \\
\tan \beta=50.0\end{array}$ \\
\hline 70.0 & 0.003 & 0.014 & 0.185 & 1.691 & 4.709 \\
\hline 80.0 & 0.003 & 0.012 & 0.202 & 1.885 & 5.260 \\
\hline 85.0 & 0.003 & 0.010 & 0.206 & 1.966 & 5.498 \\
\hline 90.0 & 0.003 & 0.007 & 0.205 & 2.060 & 5.729 \\
\hline 95.0 & 0.003 & 0.005 & 0.196 & 2.135 & 6.050 \\
\hline 100.0 & 0.004 & 0.004 & 0.170 & 2.157 & 6.217 \\
\hline 105.0 & 0.004 & - & 0.100 & 1.995 & 6.225 \\
\hline 110.0 & 0.004 & - & 0.019 & 1.200 & 5.150 \\
\hline 111.0 & 0.004 & - & 0.007 & 0.690 & 3.350 \\
\hline 111.5 & 0.004 & - & 0.004 & 0.410 & 2.680 \\
\hline 112.0 & 0.004 & - & - & 0.180 & 1.800 \\
\hline 112.5 & 0.004 & - & - & 0.031 & 0.420 \\
\hline$\sim 113.0$ & 0.004 & - & - & 0.004 & 0.004 \\
\hline
\end{tabular}

Table 10: Total decay width of the $H$-boson for $m_{\mathrm{t}}=175 \mathrm{GeV}$.

\begin{tabular}{|c||c||c|c|c|c|}
\hline$m_{\mathrm{H}}$ & $\begin{array}{c}\Gamma^{\mathrm{tot}}(\mathrm{GeV}) \\
\text { SM Higgs }\end{array}$ & $\begin{array}{c}\Gamma_{\mathrm{H}}^{\mathrm{tot}}(\mathrm{GeV}) \\
\tan \beta=3\end{array}$ & $\begin{array}{c}\Gamma_{\mathrm{H}}^{\mathrm{tot}}(\mathrm{GeV}) \\
\tan \beta=10.0\end{array}$ & $\begin{array}{c}\Gamma_{\mathrm{H}}^{\mathrm{tot}}(\mathrm{GeV}) \\
\tan \beta=30.0\end{array}$ & $\begin{array}{c}\Gamma_{\mathrm{H}}^{\mathrm{tot}}(\mathrm{GeV}) \\
\tan \beta=50.0\end{array}$ \\
\hline \hline 113.0 & 0.004 & - & 0.019 & 0.056 & 0.08 \\
114.0 & 0.004 & - & 0.015 & 0.659 & 2.50 \\
115.0 & 0.004 & - & 0.031 & 1.281 & 3.80 \\
116.0 & $\mathbf{0 . 0 0 4}$ & - & 0.048 & 1.523 & 5.37 \\
117.0 & 0.004 & 0.038 & 0.069 & 1.738 & 6.00 \\
120.0 & 0.004 & 0.005 & 0.126 & 2.291 & 6.84 \\
130.0 & 0.004 & 0.010 & 0.232 & 2.690 & 7.90 \\
150.0 & 0.02 & 0.073 & 0.392 & 3.594 & 10.00 \\
200.0 & 1.37 & 0.249 & 0.508 & 4.451 & 12.37 \\
250.0 & 4.10 & 0.268 & 0.615 & 5.298 & 14.71 \\
300.0 & 8.42 & 0.255 & 0.705 & 6.122 & 17.00 \\
350.0 & 15.6 & 0.400 & 0.807 & 6.915 & 19.20 \\
400.0 & 27.8 & 0.845 & 0.933 & 7.698 & 21.36 \\
450.0 & 45.2 & 1.335 & 1.061 & 8.472 & 23.50 \\
500.0 & 63.6 & 1.594 & 1.128 & 8.873 & 24.61 \\
\hline
\end{tabular}


Table 11: Total decay width of the A-boson for $m_{\mathrm{t}}=175 \mathrm{GeV}$.

\begin{tabular}{|c||c|c|c|c|c|}
\hline$m_{\mathrm{A}}(\mathrm{GeV})$ & $\begin{array}{c}\Gamma^{\mathrm{tot}}(\mathrm{GeV}) \\
\text { SM Higgs }\end{array}$ & $\begin{array}{c}\Gamma_{\mathrm{A}}^{\mathrm{tot}}(\mathrm{GeV}) \\
\tan \beta=3.0\end{array}$ & $\begin{array}{c}\Gamma_{\mathrm{A}}^{\mathrm{tot}}(\mathrm{GeV}) \\
\tan \beta=10.0\end{array}$ & $\begin{array}{c}\Gamma_{\mathrm{A}}^{\mathrm{tot}}(\mathrm{GeV}) \\
\tan \beta=30.0\end{array}$ & $\begin{array}{c}\Gamma_{\mathrm{A}}^{\text {tot }}(\mathrm{GeV}) \\
\tan \beta=50.0\end{array}$ \\
\hline \hline 100.0 & .004 & 0.023 & 0.252 & 2.270 & 6.305 \\
150.0 & 0.02 & 0.032 & 0.353 & 3.179 & 8.831 \\
200.0 & 1.37 & 0.043 & 0.450 & 4.050 & 11.24 \\
250.0 & 4.10 & 0.062 & 0.545 & 4.890 & 13.58 \\
300.0 & 8.42 & 0.072 & 0.637 & 5.711 & 15.86 \\
350.0 & 15.6 & 0.084 & 0.726 & 6.516 & 18.10 \\
400.0 & 27.8 & 1.402 & 0.932 & 7.321 & 20.30 \\
450.0 & 45.2 & 2.012 & 1.073 & 8.108 & 22.47 \\
500.0 & 63.6 & 2.520 & 1.204 & 8.883 & 24.62 \\
\hline
\end{tabular}

Table 12: Total decay width of the $\mathrm{H}^{ \pm}$-boson for $m_{\mathrm{t}}=175 \mathrm{GeV}$.

\begin{tabular}{|c||c|c|c|c|}
\hline$m_{\mathrm{H}^{ \pm}}(\mathrm{GeV})$ & $\begin{array}{c}\Gamma_{\mathrm{H}^{ \pm}}^{\mathrm{tot}}(\mathrm{GeV}) \\
\tan \beta=3.0\end{array}$ & $\begin{array}{c}\Gamma_{\mathrm{H}^{ \pm}}^{\mathrm{tot}}(\mathrm{GeV}) \\
\tan \beta=10.0\end{array}$ & $\begin{array}{c}\Gamma_{\mathrm{H}^{ \pm}}^{\mathrm{tot}}(\mathrm{GeV}) \\
\tan \beta=30.0\end{array}$ & $\begin{array}{c}\Gamma_{\mathrm{H}^{ \pm}}^{\mathrm{tot}}(\mathrm{GeV}) \\
\tan \beta=50.0\end{array}$ \\
\hline \hline 100.0 & 0.002 & 0.022 & 0.200 & - \\
110.0 & 0.002 & 0.024 & 0.219 & 0.605 \\
120.0 & 0.002 & 0.026 & 0.237 & 0.661 \\
130.0 & 0.002 & 0.028 & 0.256 & 0.712 \\
140.0 & 0.003 & 0.028 & 0.256 & 0.763 \\
150.0 & 0.003 & 0.030 & 0.275 & 0.817 \\
160.0 & 0.003 & 0.032 & 0.294 & 0.869 \\
170.0 & 0.004 & 0.037 & 0.333 & 1.075 \\
\hline
\end{tabular}




\section{Branching ratios, cross-sections and rates}

\section{1 h-boson}

The h-boson is the lightest of the MSSM Higgs bosons. As discussed in Section 2, the mass range of interest is from $m_{\mathrm{h}} \sim 70 \mathrm{GeV}$ (good overlap with LEP2) to the maximum allowed value of 100 to $130 \mathrm{GeV}$, depending on $m_{\mathrm{t}}$. Over this mass range, the SM Higgs boson can only be discovered through $\mathrm{H} \rightarrow \gamma \gamma$ and $\mathrm{H} \rightarrow \mathrm{b} \overline{\mathrm{b}}$ decays. Since the h-boson couplings approach the SM Higgs couplings over most of the $\left(m_{\mathrm{A}}, \tan \beta\right)$ parameter space, it is natural to explore the same decay channels for the h-boson as for the SM Higgs boson.

\subsubsection{Branching ratios}

The $\mathrm{h} \rightarrow \gamma \gamma$ decay is governed by loops of fermions, gauge bosons, charged Higgs bosons, sfermions and charginos. As discussed in Section 2 and shown in Fig. 4, the relevant couplings vary very fast for low values of $m_{\mathrm{A}}$. In particular, the coupling to $W$-boson pairs, which dominates the SM H $\rightarrow \gamma \gamma$ decays, is strongly suppressed for small values of $m_{\mathrm{A}}$. Fig. 6 shows how the $\mathrm{h} \rightarrow \gamma \gamma$ branching ratio varies as a function of $m_{\mathrm{h}}$ and $m_{\mathrm{A}}$ for different values of $\tan \beta$ and $m_{\mathrm{t}}$. The branching ratio varies rapidly as a function of $m_{\mathrm{h}}$, reaching the $\mathrm{SM}$ value as $m_{\mathrm{h}}$ reaches its maximum allowed value. This reflects directly the variation of the h-boson couplings to massive gauge bosons as a function of $m_{\mathrm{h}}$ shown in Fig. 4, where the rise is also seen to be steeper for larger values of $\tan \beta$.

The $\mathrm{h} \rightarrow \gamma \gamma$ branching ratio only slowly increases as a function of $m_{\mathrm{A}}$, since $m_{\mathrm{h}}$ varies very slowly as a function of $m_{\mathrm{A}}$, and reaches the SM value for large $m_{\mathrm{A}}{ }^{4}$. This is due to the slow saturation of the $\tau \tau$ and $\mathrm{b} \overline{\mathrm{b}}$ decay widths, whereas the $\gamma \gamma$ decay width is already saturated. The dependence on $m_{\mathrm{t}}$ reflects only the variation of the maximum allowed values of $m_{\mathrm{h}}$ as $m_{\mathrm{t}}$ varies.

The dominant $h \rightarrow b \bar{b}$ branching ratio is shown in a similar way in Fig. 7 . It varies only slowly as a function of the parameters, and is slightly enhanced with respect to the $\mathrm{SM} \mathrm{H} \rightarrow \mathrm{b} \overline{\mathrm{b}}$ branching ratio, for large values of $\tan \beta$ and not too large values of $m_{\mathrm{A}}$, due to the enhanced couplings to down-type quarks (see Fig. 4).

\footnotetext{
${ }^{4}$ Fig. 6 shows the maximum branching ratio value per mass bin and not the average.
} 

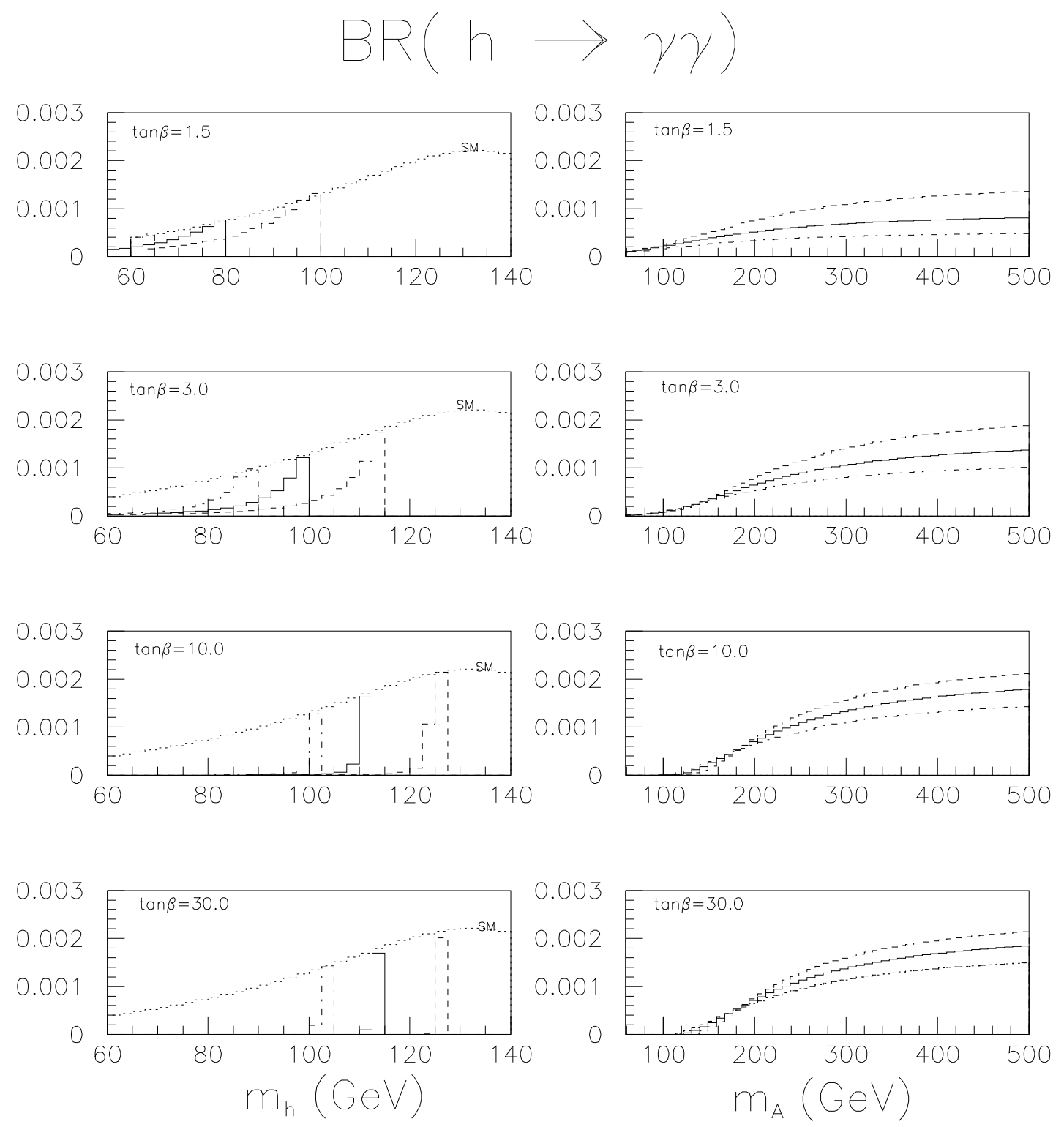

Figure 6: Branching ratio for $\mathrm{h} \rightarrow \gamma \gamma$ as a function of $m_{\mathrm{h}}$ (left) and $m_{\mathrm{A}}$ (right) for four values of $\tan \beta$. The solid line is for $m_{\mathrm{t}}=175 \mathrm{GeV}$, the dashed one for $m_{\mathrm{t}}=200 \mathrm{GeV}$ and the dot-dashed one for $m_{\mathrm{t}}=150 \mathrm{GeV}$. The figures on the left also show the SM predictions (dotted lines). 

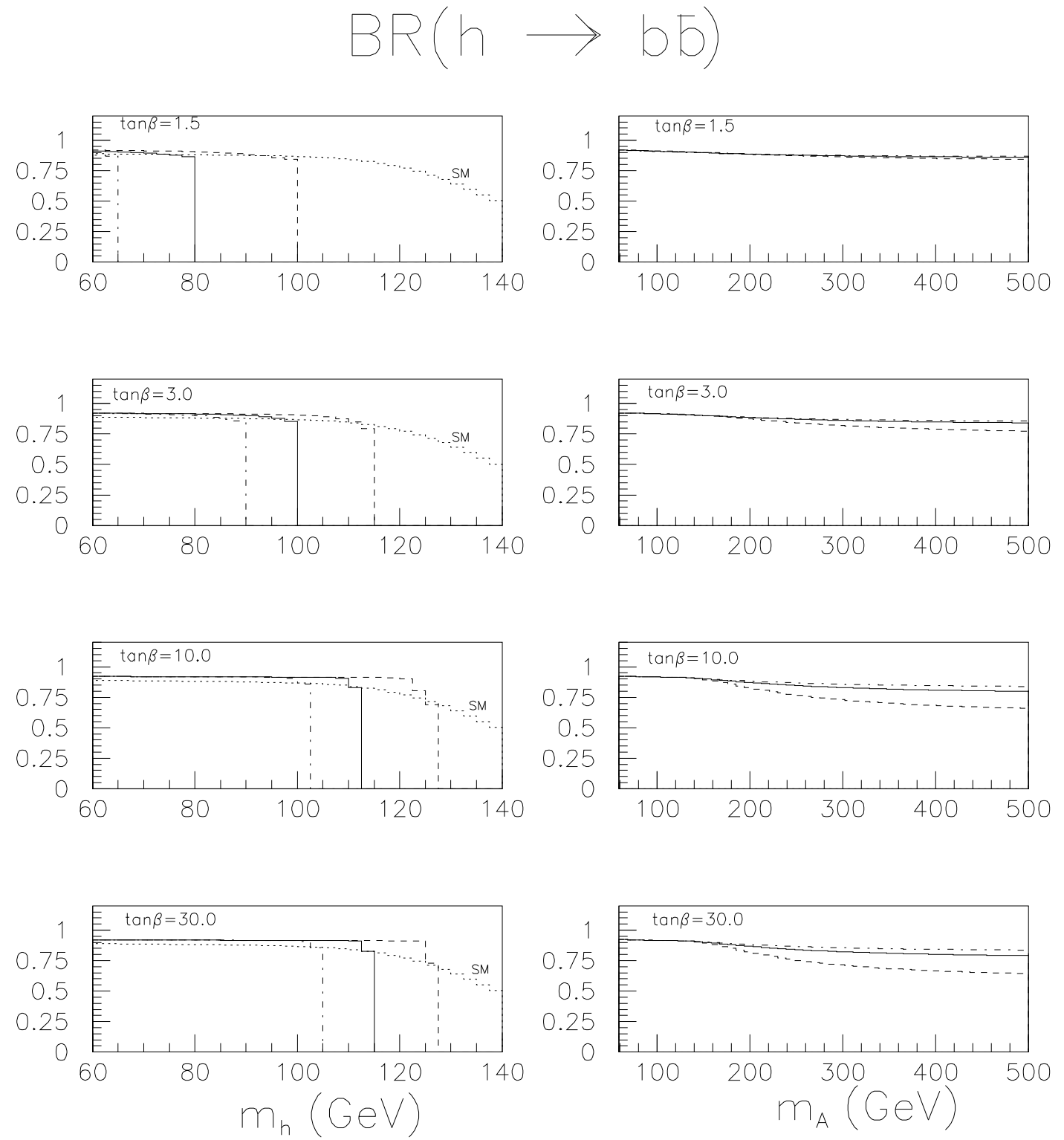

Figure 7: Branching ratio for $\mathrm{h} \rightarrow \mathrm{b} \overline{\mathrm{b}}$ as a function of $m_{\mathrm{h}}$ (left) and $m_{\mathrm{A}}$ (right) for four values of $\tan \beta$. The solid line is for $m_{\mathrm{t}}=175 \mathrm{GeV}$, the dashed one for $m_{\mathrm{t}}=200 \mathrm{GeV}$ and the dot-dashed one for $m_{\mathrm{t}}=150 \mathrm{GeV}$. The figures on the left also show the SM predictions (dotted lines). 


\subsubsection{Production cross-sections}

As discussed in detail in [3] for the SM Higgs boson, several processes contribute to Higgs-boson production. The h-boson production cross-sections are calculated for five subprocesses: $g g \rightarrow h, q q \rightarrow q q h\left(W W_{f u s}, Z Z_{f u s}\right)$,

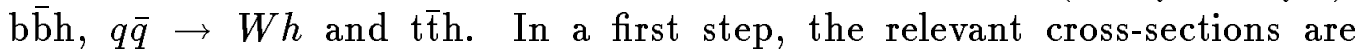
computed using PYTHIA 5.7; in a second step they are multiplied by the appropriate correction factors (in particular, the contributions to $g g \rightarrow h$ production from stop, sbottom and charged Higgs loops are included, using the calculations of $[6])$.

Fig. 8 shows the summed h-boson production cross-section for the first three subprocesses listed above, as a function of $m_{\mathrm{h}}$ and $m_{\mathrm{A}}$, for different values of $\tan \beta$ and $m_{\mathrm{t}}$. This cross-section is strongly enhanced, through the $\mathrm{b} \overline{\mathrm{b}} \mathrm{h}$ contribution, for large values of $\tan \beta$ and small values of $m_{\mathrm{A}}$, but it decreases rapidly towards the SM Higgs-boson production cross-section, as $m_{\mathrm{h}}$ increases towards its maximum allowed value. The apparent dependence on $m_{\mathrm{t}}$, shown for the cross-section as a function of $m_{\mathrm{A}}$, is only a reflection of the variation of the maximum allowed value of $m_{\mathrm{h}}$ as $m_{\mathrm{t}}$ varies.

Figs. 9 and 10 show separately the cross-sections for h-boson associated production with a $\mathrm{W}$-boson or a $t \bar{t}$-pair respectively. These cross-sections include the branching ratio for at least one $\mathrm{W} \rightarrow \ell \nu$ decay, where the lepton (electron or muon) is used to trigger the experiment. For a given value of $m_{\mathrm{h}}$, both cross-sections decrease rapidly as $\tan \beta$ increases. Both cross-sections are suppressed with respect to the corresponding SM cross-sections, and are only comparable to them if $m_{\mathrm{h}}$ is close to its maximum allowed value.

\subsubsection{Expected rates for signatures involving the h-boson}

Tables $13(g g \rightarrow h, q q \rightarrow q q h, \mathrm{~b} \overline{\mathrm{b}} \mathrm{h})$ and 14 (Wh, thth with at least one $\mathrm{W} \rightarrow \ell \nu$ decay) show the expected rates (cross-section times branching ratio: $\sigma \times B R$ ) for $\mathrm{h} \rightarrow \gamma \gamma$ decays as a function of $m_{\mathrm{h}}$, for $m_{\mathrm{t}}=175 \mathrm{GeV}$ and four different values of $\tan \beta$. The corresponding rates in the SM Higgs-boson case are also shown for comparison. As $m_{\mathrm{h}}$ gets close to its maximum allowed value, for a given choice of $\tan \beta$, the expected rates increase rapidly towards values close to the corresponding SM rates, particularly in the case of Table 13, where the rates increase by more than a factor of 2 , while $m_{\mathrm{h}}$ changes by less than $1 \mathrm{GeV}$ but $m_{\mathrm{A}}$ varies from $\sim 200 \mathrm{GeV}$ to $\sim 500 \mathrm{GeV}$. This steep dependence will have to be kept in mind when investigating the discovery potential in this particular channel. The expected rates for $m_{\mathrm{A}} \sim 500 \mathrm{GeV}$ are somewhat larger (by 10 to $20 \%$ ) than the corresponding SM rates, but this is well within the uncertainties due to the different treatment of the respective $h \rightarrow \gamma \gamma$ and SM H $\rightarrow \gamma \gamma$ decay branching ratios in terms of QCD corrections.

Tables 15 and 16, for Wh and tith associated production respectively, show the expected rates for $h \rightarrow b \bar{b}$ decays in a similar way. The same trends as in Tables 13 and 14 are observed, namely that the expected rates for a 
given choice of $\tan \beta$, are largest and approximately equal to the SM rates, for $m_{\mathrm{h}}$ close to its maximum allowed value. The contribution to $h \rightarrow b \bar{b}$ decays from Wh associated production is about $20 \%$ smaller than that from t'th associated production, for $m_{\mathrm{t}}=175 \mathrm{GeV}$ and for $m_{\mathrm{h}}$ at its maximum allowed value.

Table 13: Expected rates $(\sigma \times B R)$ for $\mathrm{h} \rightarrow \gamma \gamma$ decays $(g g \rightarrow h, q q \rightarrow q q h$, $\mathrm{b} \overline{\mathrm{bh}}$ ) as a function of $m_{\mathrm{h}}$, for $m_{\mathrm{t}}=175 \mathrm{GeV}$ and four different values of $\tan \beta$. The corresponding rates in the SM case are taken from Table 7 of $[3]$.

\begin{tabular}{|c|c|c|c|c|c|}
\hline \multirow[t]{3}{*}{$m_{\mathrm{h}}(\mathrm{GeV})$} & \multicolumn{5}{|c|}{$\sigma \times B R(\mathrm{fb})$} \\
\hline & SM Higgs & \multicolumn{4}{|c|}{ MSSM $h$-boson } \\
\hline & & $\tan \beta=3.0$ & $\tan \beta=10.0$ & $\tan \beta=30.0$ & $\tan \beta=50.0$ \\
\hline 70.0 & 27.6 & $2.1\left(m_{\mathrm{A}} \sim 85\right)$ & $0.7\left(m_{\mathrm{A}} \sim 72\right)$ & $3.2\left(m_{\mathrm{A}} \sim 70\right)$ & $8.7\left(m_{\mathrm{A}} \sim 70\right)$ \\
\hline 80.0 & 32.3 & $3.4\left(m_{\mathrm{A}} \sim 105\right)$ & $0.6\left(m_{\mathrm{A}} \sim 82\right)$ & $1.9\left(m_{\mathrm{A}} \sim 80\right)$ & $5.0\left(m_{\mathrm{A}} \sim 80\right)$ \\
\hline 90.0 & 36.6 & $8.3\left(m_{\mathrm{A}} \sim 140\right)$ & $0.7\left(m_{\mathrm{A}} \sim 93\right)$ & $1.1\left(m_{\mathrm{A}} \sim 90\right)$ & $3.8\left(m_{\mathrm{A}} \sim 90\right)$ \\
\hline 95.0 & 38.2 & $18.6\left(m_{\mathrm{A}} \sim 190\right)$ & $0.8\left(m_{\mathrm{A}} \sim 100\right)$ & $1.0\left(m_{\mathrm{A}} \sim 95\right)$ & $2.8\left(m_{\mathrm{A}} \sim 90\right)$ \\
\hline 100.0 & 40.7 & $46.3\left(m_{\mathrm{A}} \sim 500\right)$ & $0.9\left(m_{\mathrm{A}} \sim 105\right)$ & $0.9\left(m_{\mathrm{A}} \sim 100\right)$ & $1.9\left(m_{\mathrm{A}} \sim 100\right)$ \\
\hline 105.0 & 42.5 & - & $1.5\left(m_{\mathrm{A}} \sim 120\right)$ & $1.0\left(m_{\mathrm{A}} \sim 106\right)$ & $1.7\left(m_{\mathrm{A}} \sim 106\right)$ \\
\hline 110.0 & 44.7 & - & $8.8\left(m_{\mathrm{A}} \sim 150\right)$ & $1.6\left(m_{\mathrm{A}} \sim 113\right)$ & $2.0\left(m_{\mathrm{A}} \sim 111\right)$ \\
\hline 110.5 & & - & $13.9\left(m_{\mathrm{A}} \sim 170\right)$ & $1.6\left(m_{\mathrm{A}} \sim 115\right)$ & $2.2\left(m_{\mathrm{A}} \sim 112\right)$ \\
\hline 111.0 & & - & $22.7\left(m_{\mathrm{A}} \sim 220\right)$ & $1.7\left(m_{\mathrm{A}} \sim 116\right)$ & $2.0\left(m_{\mathrm{A}} \sim 113\right)$ \\
\hline 111.5 & & - & $45.6\left(m_{\mathrm{A}} \sim 500\right)$ & $1.8\left(m_{\mathrm{A}} \sim 119\right)$ & $2.1\left(m_{\mathrm{A}} \sim 114\right)$ \\
\hline 112.0 & & - & - & $2.1\left(m_{\mathrm{A}} \sim 120\right)$ & $2.1\left(m_{\mathrm{A}} \sim 115\right)$ \\
\hline 112.5 & & - & - & $5.2\left(m_{\mathrm{A}} \sim 140\right)$ & $2.0\left(m_{\mathrm{A}} \sim 118\right)$ \\
\hline 112.6 & & - & - & $9.0\left(m_{\mathrm{A}} \sim 160\right)$ & $2.1\left(m_{\mathrm{A}} \sim 120\right)$ \\
\hline 112.7 & & - & - & $21.6\left(m_{\mathrm{A}} \sim 210\right)$ & $2.2\left(m_{\mathrm{A}} \sim 122\right)$ \\
\hline 112.8 & & 一 & - & $47.1\left(m_{\mathrm{A}} \sim 500\right)$ & $2.4\left(m_{\mathrm{A}} \sim 126\right)$ \\
\hline 112.9 & & - & 一 & - & $4.1\left(m_{\mathrm{A}} \sim 137\right)$ \\
\hline 113.0 & & - & - & - & $23.0\left(m_{\mathrm{A}} \sim 210\right)$ \\
\hline 113.1 & & - & - & - & $51.0\left(m_{\mathrm{A}} \sim 500\right)$ \\
\hline
\end{tabular}




\section{$\sigma(g g, W W, Z Z, b b h)(p b)$}
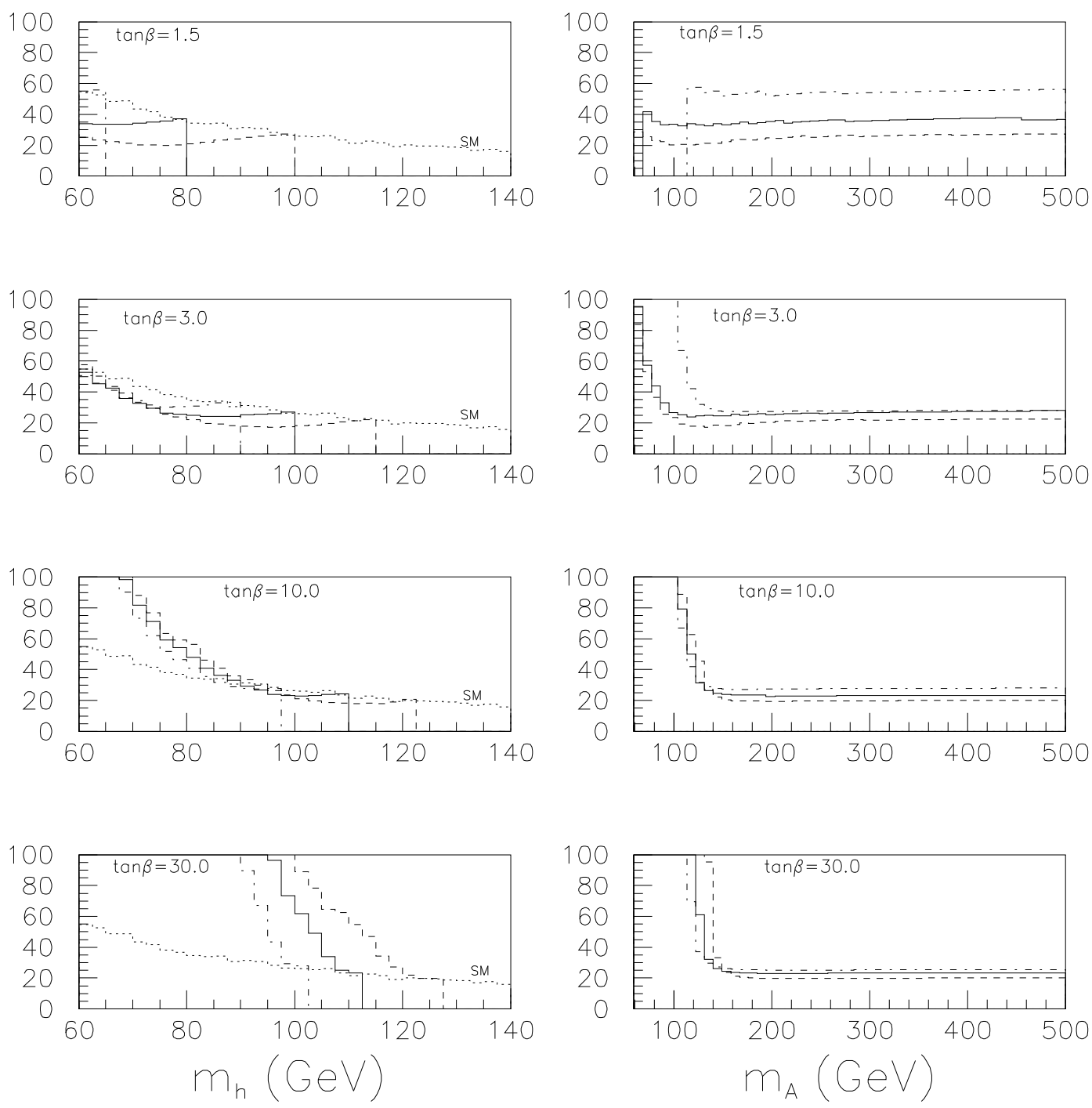

Figure 8: Cross-section for h-boson production $(g g \rightarrow h, q q \rightarrow q q h$, and $\mathrm{b} \overline{\mathrm{b} h}$ ) as a function of $m_{\mathrm{h}}$ (left) and $m_{\mathrm{A}}$ (right) for four values of $\tan \beta$. The solid line is for $m_{\mathrm{t}}=175 \mathrm{GeV}$, the dashed one for $m_{\mathrm{t}}=200 \mathrm{GeV}$ and the dot-dashed one for $m_{\mathrm{t}}=150 \mathrm{GeV}$. The figures on the left also show the SM predictions (dotted lines). 

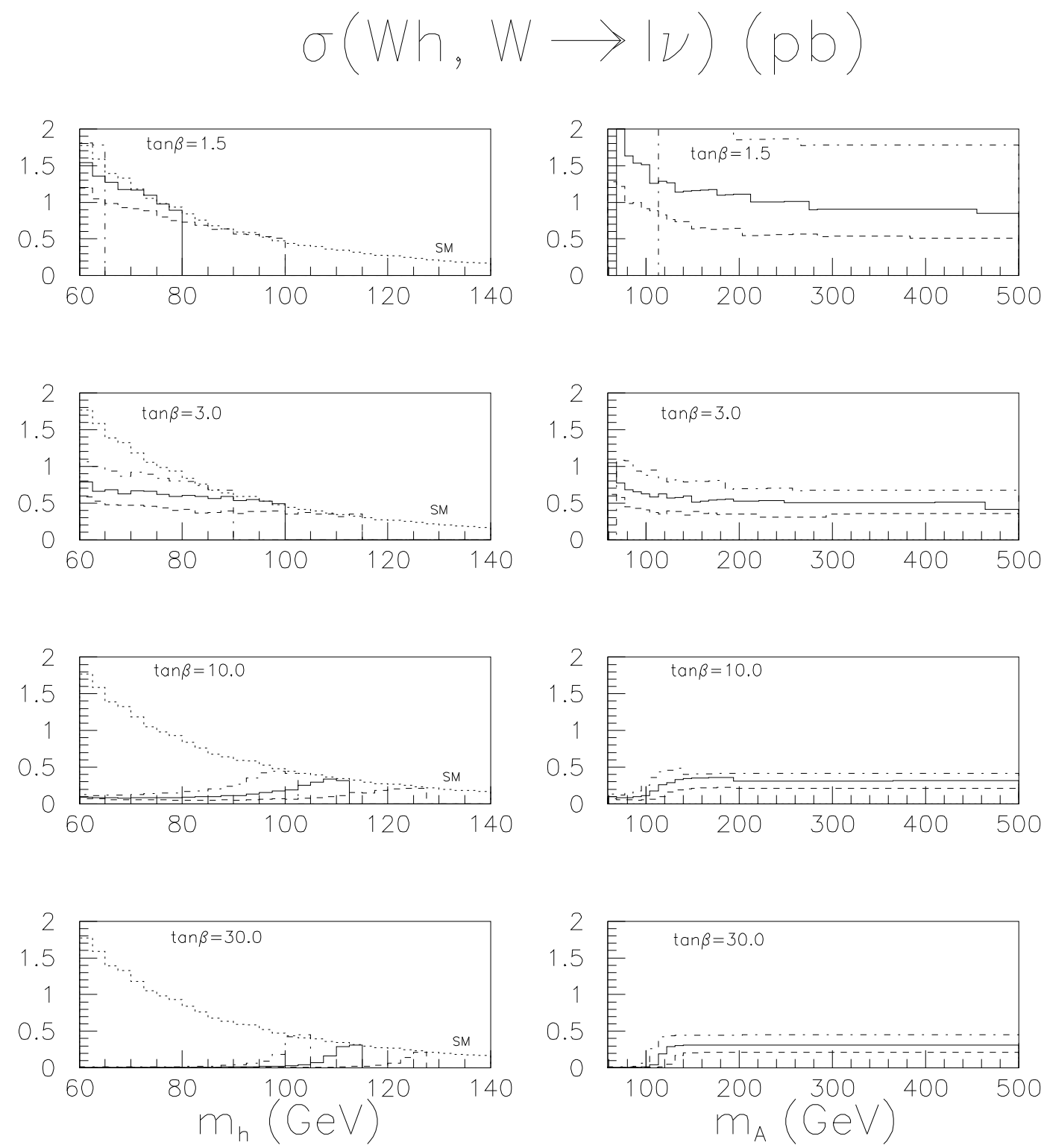

Figure 9: Cross-section for associated production of an h-boson (Wh with $\mathrm{W} \rightarrow \ell \nu$ decay) as a function of $m_{\mathrm{h}}$ (left) and $m_{\mathrm{A}}$ (right) for four values of $\tan \beta$. The solid line is for $m_{\mathrm{t}}=175 \mathrm{GeV}$, the dashed one for $m_{\mathrm{t}}=200 \mathrm{GeV}$ and the dot-dashed one for $m_{\mathrm{t}}=150 \mathrm{GeV}$. The figures on the left also show the SM predictions (dotted lines). 


\section{$\sigma(t t h, W \rightarrow \mid \nu)(p b)$}
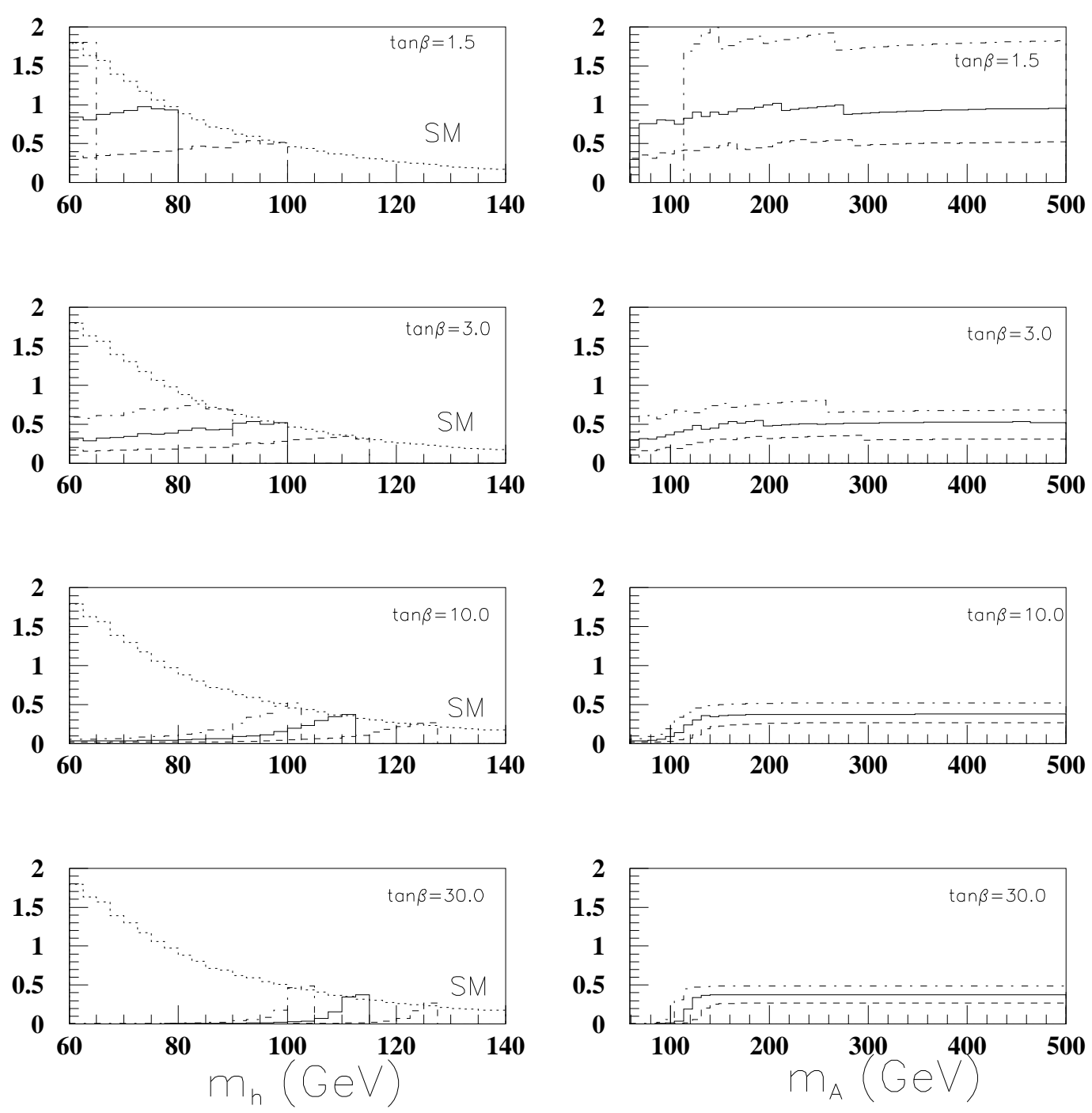

Figure 10: Cross-section for associated production of an h-boson (tth with at least one $\mathrm{W} \rightarrow \ell \nu$ decay) as a function of $m_{\mathrm{h}}$ (left) and $m_{\mathrm{A}}$ (right) for four values of $\tan \beta$. The solid line is for $m_{\mathrm{t}}=175 \mathrm{GeV}$, the dashed one for $m_{\mathrm{t}}=200 \mathrm{GeV}$ and the dot-dashed one for $m_{\mathrm{t}}=150 \mathrm{GeV}$. The figures on the left also show the SM predictions for $m_{\mathrm{t}}=175 \mathrm{GeV}$ (dotted lines). 
Table 14: Expected rates $(\sigma \times B R)$ for $\mathrm{h} \rightarrow \gamma \gamma$ decays (Wh, t th with at least one $\mathrm{W} \rightarrow \ell \nu$ decay) as a function of $m_{\mathrm{h}}$, for $m_{\mathrm{t}}=175 \mathrm{GeV}$ and four different values of $\tan \beta$. The corresponding rates for the $S M$ case are also shown for $m_{\mathrm{t}}=175 \mathrm{GeV}$.

\begin{tabular}{|c|c|c|c|c|c|}
\hline \multirow[t]{3}{*}{$m_{\mathrm{h}}(\mathrm{GeV})$} & \multicolumn{5}{|c|}{$\sigma \times B R(\mathrm{fb})$} \\
\hline & SM Higgs & \multicolumn{4}{|c|}{ MSSM $h$-boson } \\
\hline & & $\tan \beta=3.0$ & $\tan \beta=10.0$ & $\tan \beta=30.0$ & $\tan \beta=50.0$ \\
\hline 70.0 & & $0.06\left(m_{\mathrm{A}} \sim 85\right)$ & $<0.01\left(m_{\mathrm{A}} \sim 72\right)$ & $<0.01\left(m_{\mathrm{A}} \sim 70\right)$ & $<0.01\left(m_{\mathrm{A}} \sim 70\right)$ \\
\hline 80.0 & 1.17 & $0.12\left(m_{\mathrm{A}} \sim 105\right)$ & $<0.01\left(m_{\mathrm{A}} \sim 82\right)$ & $<0.01\left(m_{\mathrm{A}} \sim 80\right)$ & $<0.01\left(m_{\mathrm{A}} \sim 80\right)$ \\
\hline 90.0 & 1.04 & $0.34\left(m_{\mathrm{A}} \sim 140\right)$ & $<0.01\left(m_{\mathrm{A}} \sim 93\right)$ & $<0.01\left(m_{\mathrm{A}} \sim 90\right)$ & $<0.01\left(m_{\mathrm{A}} \sim 90\right)$ \\
\hline 95.0 & 1.07 & $0.73\left(m_{\mathrm{A}} \sim 190\right)$ & $<0.01\left(m_{\mathrm{A}} \sim 100\right)$ & $<0.01\left(m_{\mathrm{A}} \sim 95\right)$ & $<0.01\left(m_{\mathrm{A}} \sim 90\right)$ \\
\hline 100.0 & 1.10 & $1.40\left(m_{\mathrm{A}} \sim 500\right)$ & $<0.01\left(m_{\mathrm{A}} \sim 105\right)$ & $<0.01\left(m_{\mathrm{A}} \sim 100\right)$ & $<0.01\left(m_{\mathrm{A}} \sim 100\right)$ \\
\hline 105.0 & 1.00 & - & $0.03\left(m_{\mathrm{A}} \sim 120\right)$ & $<0.01\left(m_{\mathrm{A}} \sim 106\right)$ & $<0.01\left(m_{\mathrm{A}} \sim 106\right)$ \\
\hline 110.0 & 0.90 & - & $0.21\left(m_{\mathrm{A}} \sim 150\right)$ & $<0.01\left(m_{\mathrm{A}} \sim 113\right)$ & $<0.01\left(m_{\mathrm{A}} \sim 111\right)$ \\
\hline 110.5 & & - & $0.37\left(m_{\mathrm{A}} \sim 170\right)$ & $<0.01\left(m_{\mathrm{A}} \sim 115\right)$ & $<0.01\left(m_{\mathrm{A}} \sim 112\right)$ \\
\hline 111.0 & & - & $0.67\left(m_{\mathrm{A}} \sim 220\right)$ & $<0.01\left(m_{\mathrm{A}} \sim 116\right)$ & $<0.01\left(m_{\mathrm{A}} \sim 113\right)$ \\
\hline 111.5 & & - & $1.34\left(m_{\mathrm{A}} \sim 500\right)$ & $0.01\left(m_{\mathrm{A}} \sim 119\right)$ & $<0.01\left(m_{\mathrm{A}} \sim 114\right)$ \\
\hline 112.0 & & - & - & $0.01\left(m_{\mathrm{A}} \sim 120\right)$ & $<0.01\left(m_{\mathrm{A}} \sim 115\right)$ \\
\hline 112.5 & & - & 一 & $0.11\left(m_{\mathrm{A}} \sim 140\right)$ & $<0.01\left(m_{\mathrm{A}} \sim 118\right)$ \\
\hline 112.6 & & - & - & $0.27\left(m_{\mathrm{A}} \sim 160\right)$ & $0.01\left(m_{\mathrm{A}} \sim 120\right)$ \\
\hline 112.7 & & - & 一 & $0.64\left(m_{\mathrm{A}} \sim 210\right)$ & $0.02\left(m_{\mathrm{A}} \sim 122\right)$ \\
\hline 112.8 & & - & - & $1.40\left(m_{\mathrm{A}} \sim 500\right)$ & $0.04\left(m_{\mathrm{A}} \sim 126\right)$ \\
\hline 112.9 & & - & 一 & - & $0.10\left(m_{\mathrm{A}} \sim 137\right)$ \\
\hline 113.0 & & - & - & - & $0.63\left(m_{\mathrm{A}} \sim 210\right)$ \\
\hline 113.1 & & - & - & - & $1.36\left(m_{\mathrm{A}} \sim 500\right)$ \\
\hline
\end{tabular}


Table 15: Expected rates $(\sigma \times B R$ ) for $\mathrm{h} \rightarrow \mathrm{b} \overline{\mathrm{b}}$ decays (Wh with $\mathrm{W} \rightarrow \ell \nu$ decay) as a function of $m_{\mathrm{h}}$, for $m_{\mathrm{t}}=175 \mathrm{GeV}$ and four different values of $\tan \beta$. The corresponding rates for the SM case are also shown.

\begin{tabular}{|c|c|c|c|c|c|}
\hline \multirow{3}{*}{$m_{\mathrm{h}}(\mathrm{GeV})$} & \multicolumn{5}{|c|}{$\sigma \times B R(\mathrm{fb})$} \\
\hline & SM Higgs & \multicolumn{4}{|c|}{ MSSM $h$-boson } \\
\hline & & $\tan \beta=1.5$ & $\tan \beta=3.0$ & $\tan \beta=10.0$ & $\tan \beta=30.0$ \\
\hline 70.0 & & $1038\left(m_{\mathrm{A}} \sim 145\right)$ & $597\left(m_{\mathrm{A}} \sim 85\right)$ & $76\left(m_{\mathrm{A}} \sim 72\right)$ & $8\left(m_{\mathrm{A}} \sim 70\right)$ \\
\hline 80.0 & 769 & $730\left(m_{\mathrm{A}} \sim 500\right)$ & $538\left(m_{\mathrm{A}} \sim 105\right)$ & $78\left(m_{\mathrm{A}} \sim 82\right)$ & $9\left(m_{\mathrm{A}} \sim 80\right)$ \\
\hline 90.0 & 567 & - & $539\left(m_{\mathrm{A}} \sim 140\right)$ & $108\left(m_{\mathrm{A}} \sim 93\right)$ & $13\left(m_{\mathrm{A}} \sim 90\right)$ \\
\hline 95.0 & & - & $491\left(m_{\mathrm{A}} \sim 190\right)$ & $134\left(m_{\mathrm{A}} \sim 100\right)$ & $16\left(m_{\mathrm{A}} \sim 95\right)$ \\
\hline 100.0 & 405 & - & $349\left(m_{\mathrm{A}} \sim 500\right)$ & $174\left(m_{\mathrm{A}} \sim 105\right)$ & $25\left(m_{\mathrm{A}} \sim 100\right)$ \\
\hline 105.0 & & - & - & $277\left(m_{\mathrm{A}} \sim 120\right)$ & $50\left(m_{\mathrm{A}} \sim 106\right)$ \\
\hline 110.0 & 300 & - & - & $316\left(m_{\mathrm{A}} \sim 150\right)$ & $178\left(m_{\mathrm{A}} \sim 113\right)$ \\
\hline 110.5 & & - & - & $318\left(m_{\mathrm{A}} \sim 170\right)$ & $228\left(m_{\mathrm{A}} \sim 115\right)$ \\
\hline 111.0 & & - & - & $268\left(m_{\mathrm{A}} \sim 220\right)$ & $214\left(m_{\mathrm{A}} \sim 116\right)$ \\
\hline 111.5 & & - & - & $250\left(m_{\mathrm{A}} \sim 500\right)$ & $248\left(m_{\mathrm{A}} \sim 119\right)$ \\
\hline 112.0 & & - & - & - & $255\left(m_{\mathrm{A}} \sim 120\right)$ \\
\hline 112.5 & & - & - & - & $280\left(m_{\mathrm{A}} \sim 140\right)$ \\
\hline 112.6 & & - & - & - & $278\left(m_{\mathrm{A}} \sim 160\right)$ \\
\hline 112.7 & & - & - & - & $269\left(m_{\mathrm{A}} \sim 210\right)$ \\
\hline 112.8 & & - & - & - & $247\left(m_{\mathrm{A}} \sim 500\right)$ \\
\hline
\end{tabular}


Table 16: Expected rates $(\sigma \times B R)$ for $\mathrm{h} \rightarrow \mathrm{b} \overline{\mathrm{b}}$ decays ( $\mathrm{t} \overline{\mathrm{t}} \mathrm{h}$ with at least one $\mathrm{W} \rightarrow \ell \nu$ decay) as a function of $m_{\mathrm{h}}$, for $m_{\mathrm{t}}=175 \mathrm{GeV}$ and four different values of $\tan \beta$. The corresponding rates in the $S M$ case are also shown for $m_{\mathrm{t}}=175 \mathrm{GeV}$.

\begin{tabular}{|c||c||c|c|c|c|}
\hline \multicolumn{1}{|c||}{$m_{\mathrm{h}}(\mathrm{GeV})$} & \multicolumn{6}{|c|}{$\sigma \times B R(\mathrm{fb})$} \\
\hline & SM Higgs & \multicolumn{5}{c|}{ MSSM $h$-boson } \\
\hline & & $\tan \beta=1.5$ & $\tan \beta=3.0$ & $\tan \beta=10.0$ & $\tan \beta=30.0$ \\
\hline \hline 70.0 & & $822\left(m_{\mathrm{A}} \sim 145\right)$ & $316\left(m_{\mathrm{A}} \sim 85\right)$ & $32\left(m_{\mathrm{A}} \sim 72\right)$ & $4\left(m_{\mathrm{A}} \sim 70\right)$ \\
80.0 & \multirow{3}{*}{731} & $809\left(m_{\mathrm{A}} \sim 500\right)$ & $366\left(m_{\mathrm{A}} \sim 105\right)$ & $43\left(m_{\mathrm{A}} \sim 82\right)$ & $5\left(m_{\mathrm{A}} \sim 80\right)$ \\
90.0 & & - & $399\left(m_{\mathrm{A}} \sim 140\right)$ & $63\left(m_{\mathrm{A}} \sim 93\right)$ & $7\left(m_{\mathrm{A}} \sim 90\right)$ \\
95.0 & & - & $483\left(m_{\mathrm{A}} \sim 190\right)$ & $94\left(m_{\mathrm{A}} \sim 100\right)$ & $12\left(m_{\mathrm{A}} \sim 95\right)$ \\
100.0 & \multirow{3}{*}{390} & - & $432\left(m_{\mathrm{A}} \sim 500\right)$ & $138\left(m_{\mathrm{A}} \sim 105\right)$ & $19\left(m_{\mathrm{A}} \sim 100\right)$ \\
105.0 & & - & - & $265\left(m_{\mathrm{A}} \sim 120\right)$ & $45\left(m_{\mathrm{A}} \sim 106\right)$ \\
110.0 & \multirow{3}{*}{300} & - & - & $318\left(m_{\mathrm{A}} \sim 150\right)$ & $174\left(m_{\mathrm{A}} \sim 113\right)$ \\
110.5 & & - & - & $324\left(m_{\mathrm{A}} \sim 170\right)$ & $228\left(m_{\mathrm{A}} \sim 115\right)$ \\
111.0 & & - & - & $316\left(m_{\mathrm{A}} \sim 220\right)$ & $246\left(m_{\mathrm{A}} \sim 116\right)$ \\
111.5 & & - & - & $298\left(m_{\mathrm{A}} \sim 500\right)$ & $289\left(m_{\mathrm{A}} \sim 119\right)$ \\
112.0 & & - & - & - & $297\left(m_{\mathrm{A}} \sim 120\right)$ \\
112.5 & & - & - & - & $332\left(m_{\mathrm{A}} \sim 140\right)$ \\
112.6 & & - & - & - & $331\left(m_{\mathrm{A}} \sim 160\right)$ \\
112.7 & & - & - & - & $318\left(m_{\mathrm{A}} \sim 220\right)$ \\
112.8 & & - & - & - & $295\left(m_{\mathrm{A}} \sim 500\right)$ \\
\hline
\end{tabular}




\subsection{H-boson}

The H-boson is the heavier of the CP-even MSSM neutral Higgs bosons: the mass range of interest is from 110 to $500 \mathrm{GeV}$ (see Section 2). Over this mass range, the SM Higgs boson can be discovered mainly through $\mathrm{H} \rightarrow \mathrm{ZZ}^{(\star)} \rightarrow 4 \ell$ decays, but also through $\mathrm{H} \rightarrow \gamma \gamma$ decays at the lower end of the mass spectrum. However, in the case of the MSSM H-boson, the spectrum of decay channels of interest is much richer and varies rapidly with $m_{\mathrm{A}}$ and with $\tan \beta$. This is due to the strong suppression of the HZZ coupling (see Fig. 5), which enhances the branching ratios to other decay channels, such as $\mathrm{H} \rightarrow \tau \tau$ and $\mathrm{H} \rightarrow \mathrm{t} \overline{\mathrm{t}}$. Moreover, the $\mathrm{H} \rightarrow \mathrm{hh}$ channel, which does not exist in the SM, has a large branching ratio over a large range of $m_{\mathrm{H}}$. This channel is of particular interest, since it would provide a simultaneous discovery of the $h$ and $\mathrm{H}$-bosons.

\subsubsection{Branching ratios}

The $\mathrm{H} \rightarrow \gamma \gamma$ branching ratio is shown in Fig. 11 as a function of $m_{\mathrm{H}}$ (left side) and of $m_{\mathrm{A}}$ (right side), for different values of $m_{\mathrm{t}}$ and $\tan \beta$. For a fixed value of $\tan \beta$, this branching ratio decreases very rapidly as $m_{\mathrm{H}}$ increases. The reason for this behaviour arises from the strong suppression, as $m_{\mathrm{H}}$ increases, of the HWW coupling, which dominates the $\mathrm{H} \rightarrow \gamma \gamma$ branching ratio and is proportional to $\cos ^{2}(\beta-\alpha)$ (see Fig. 5). The dependence on $m_{\mathrm{t}}$ just reflects the increase of the minimum allowed value of $m_{\mathrm{H}}$ as $m_{\mathrm{t}}$ increases.

The MSSM H $\rightarrow \mathrm{ZZ}^{(\star)} \rightarrow 4 \ell$ branching ratio has a complicated structure, as shown in Fig. 12. Its main feature is that it is strongly suppressed with respect to the $\mathrm{SM}$ branching ratio, but it also varies rapidly with $\tan \beta$. As shown below, for small values of $\tan \beta$ and for $2 m_{\mathrm{h}}<m_{\mathrm{H}}<2 m_{\mathrm{t}}$, the $\mathrm{H} \rightarrow$ hh decay branching ratio is dominant and, as a consequence, the $\mathrm{H} \rightarrow \mathrm{ZZ}^{(\star)} \rightarrow 4 \ell$ branching ratio is even more suppressed in this case. It is in addition quite sensitive to the value of $m_{\mathrm{t}}$ and increases significantly as $m_{\mathrm{t}}$ increases. Since the $\mathrm{H} \rightarrow \mathrm{t} \overline{\mathrm{t}}$ decay channel suppresses very strongly the $\mathrm{H} \rightarrow \mathrm{ZZ}^{(\star)} \rightarrow 4 \ell$ mode (for small values of $\tan \beta$ ), the threshold at which the $\mathrm{H} \rightarrow \mathrm{t} \overline{\mathrm{t}}$ channel opens up increases as $m_{\mathrm{t}}$ increases, and thus results in a wider region of the $\left(m_{\mathrm{A}}, \tan \beta\right)$ plane where the $\mathrm{H} \rightarrow \mathrm{ZZ}^{(*)} \rightarrow 4 \ell$ mode could be observable. Finally, this channel is also of interest for values of $\tan \beta$ smaller than 1 , in a region theoretically disfavoured but not accessible to LEP. For $\tan \beta<1.0$, the $\mathrm{H} \rightarrow \mathrm{ZZ}^{(\star)} \rightarrow 4 \ell$ branching ratio grows rapidly as $\tan \beta$ decreases, as shown in Fig. 13.

The $\mathrm{H} \rightarrow \tau \tau$ branching ratio is in most cases larger than the $\mathrm{SM}$ one, particularly for large values of $m_{\mathrm{H}}$, due to the suppressed couplings to gauge bosons in the MSSM case, and for large values of $\tan \beta$, due to the enhanced $\mathrm{H} \tau \tau$ coupling (see Fig. 5). As shown in Fig. 14, for $m_{\mathrm{H}}>150 \mathrm{GeV}$ and for large values of $\tan \beta$, the $\mathrm{H} \rightarrow \tau \tau$ branching ratio is almost independent of $m_{\mathrm{H}}$ and $m_{\mathrm{t}}$. 
The $\tau \tau$ and $\mu \mu$ decay modes are subject to the same MSSM correction factors to the couplings, so their branching ratios are in the ratio $\left(m_{\tau} / m_{\mu}\right)^{2}$, as in the SM case. The branching ratio in the $\mu \mu$ channel is therefore nearly three orders of magnitude smaller than the $\tau \tau$ branching ratio, but presents the same features, in particular at large values of $\tan \beta$.

As shown in Fig. 15, the $\mathrm{H} \rightarrow \mathrm{t} \overline{\mathrm{t}}$ channel is the dominant one for low values of $\tan \beta$ and for $m_{\mathrm{H}}>2 m_{\mathrm{t}}$. This arises again from the strongly suppressed $\mathrm{H}$-boson couplings to gauge bosons. As $\tan \beta$ increases, the $\mathrm{H} \rightarrow \mathrm{t} \overline{\mathrm{t}}$ channel competes with the $\mathrm{H} \rightarrow \mathrm{b} \overline{\mathrm{b}}$ decay channel, and its branching ratio is reduced to less than $10 \%$ for $\tan \beta=10$.

This short but necessary review of the H-boson branching ratios would not be complete without a discussion of the $\mathrm{H} \rightarrow$ hh decay mode. As shown in Fig. 16, this decay mode, unique to the MSSM H-boson, is dominant for $2 m_{\mathrm{h}}<m_{\mathrm{H}}<2 m_{\mathrm{t}}$ and for small values of $\tan \beta$, since the Hhh coupling is proportional to $\cos 2 \alpha \sin (\beta+\alpha)$. The dependence on $m_{\mathrm{t}}$ just reflects the variation of $m_{\mathrm{h}}$ with $m_{\mathrm{t}}$ at a given point in the $\left(m_{\mathrm{A}}, \tan \beta\right)$ plane. One of the most promising signatures for the $\mathrm{H} \rightarrow$ hh channel is $\mathrm{H} \rightarrow \mathrm{hh} \rightarrow \mathrm{b} \overline{\mathrm{b}} \gamma \gamma$. The $\mathrm{H} \rightarrow \mathrm{hh} \rightarrow \mathrm{b} \overline{\mathrm{b}} \gamma \gamma$ branching ratio also reflects the shapes of the $\mathrm{h} \rightarrow \mathrm{b} \overline{\mathrm{b}}$ and $\mathrm{h} \rightarrow \gamma \gamma$ branching ratios, as can be seen by comparing Fig. 16 and Fig. 17 .

\subsubsection{Production cross-sections}

As discussed already for the h-boson, several processes contribute to Higgs boson production at the $\mathrm{LHC}$. The $\mathrm{H}$-boson production cross-section is usually calculated as the sum of contributions from the following subprocesses: $g g \rightarrow H, q q \rightarrow q q H\left(W W_{f u s}, Z Z_{f u s}\right)$ and $\mathrm{b} \bar{b} H$. Fig. 18 shows this sum for four values of $\tan \beta$ and for three values of $m_{\mathrm{t}}$. It can be clearly seen that the contribution from the $\mathrm{b} \overline{\mathrm{b}} \mathrm{H}$ channel is strongly enhanced for large values of $\tan \beta$ (note the differences in vertical scale). Over the region of low values of $m_{\mathrm{H}}$ shown in Fig. 19, the variation of the H-boson production cross-section as a function of $m_{\mathrm{t}}$ merely reflects the variation of $m_{\mathrm{H}}$ versus $m_{\mathrm{t}}$. Finally, Fig. 20 shows the H-boson production cross-section for values of $\tan \beta$ lower than 1 , for which the MSSM predictions are also enhanced with respect to the SM ones. 


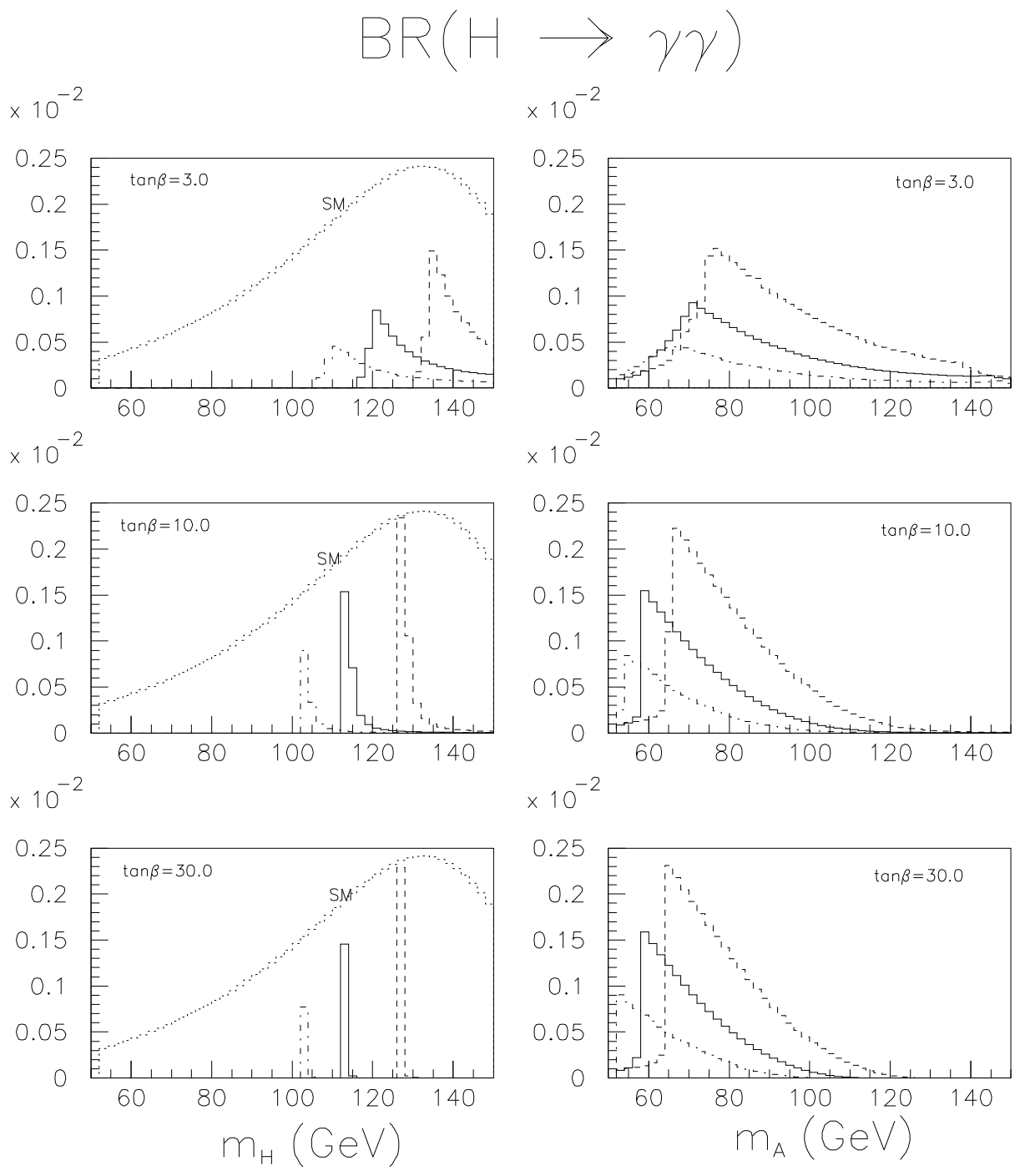

Figure 11: $\mathrm{H} \rightarrow \gamma \gamma$ branching ratio as a function of $m_{\mathrm{H}}$ (left) and $m_{\mathrm{A}}$ (right) for three values of $\tan \beta$. The solid line is for $m_{\mathrm{t}}=175 \mathrm{GeV}$, the dashed one for $m_{\mathrm{t}}=200 \mathrm{GeV}$ and the dot-dashed one for $m_{\mathrm{t}}=150 \mathrm{GeV}$. The figures on the left also show the SM predictions (dotted lines). 

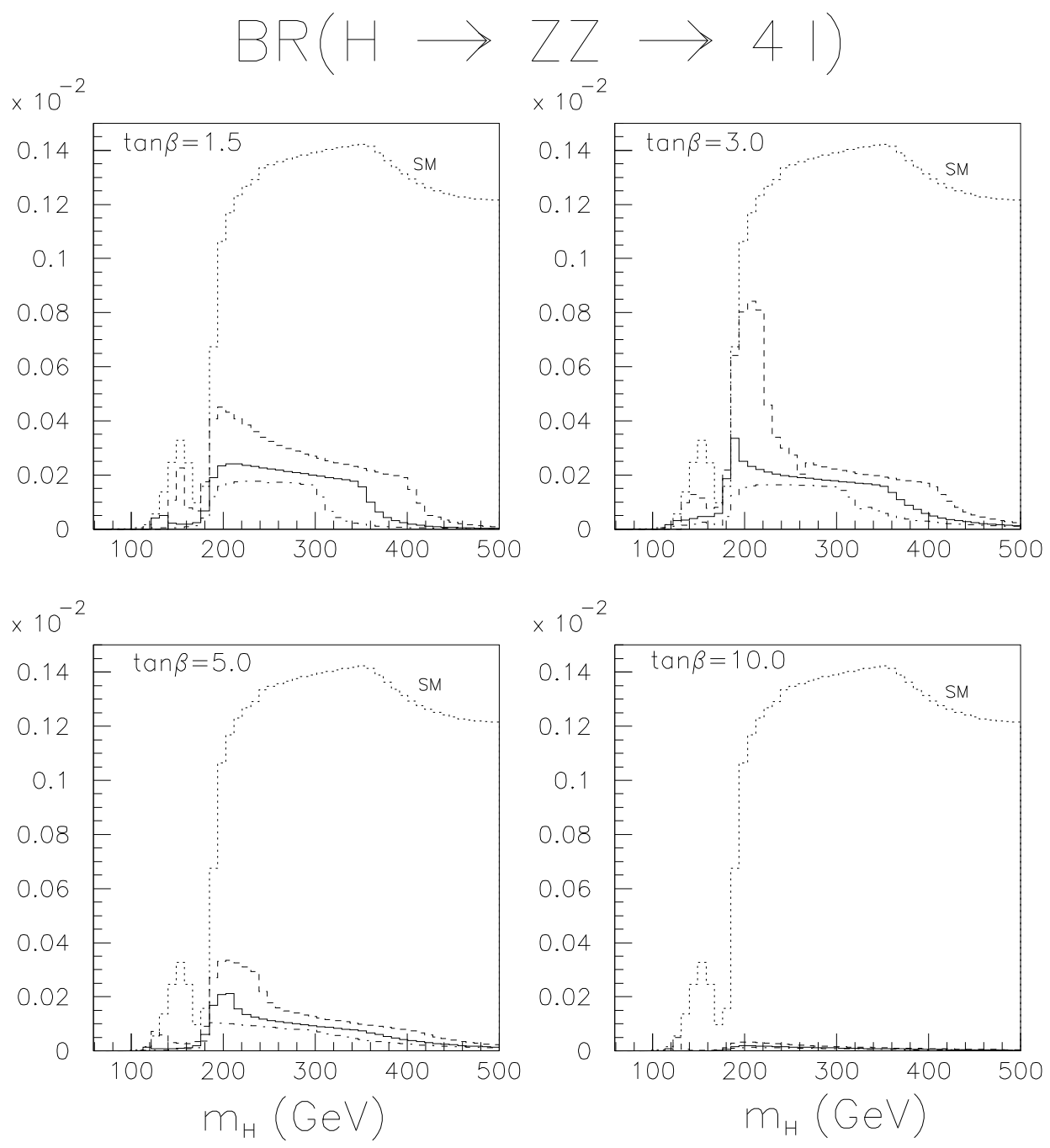

Figure 12: $\mathrm{H} \rightarrow \mathrm{ZZ}^{(\star)} \rightarrow 4 \ell$ branching ratio as a function of $m_{\mathrm{H}}$ for four values of $\tan \beta$. The solid line is for $m_{\mathrm{t}}=175 \mathrm{GeV}$, the dashed one for $m_{\mathrm{t}}=200 \mathrm{GeV}$ and the dot-dashed one for $m_{\mathrm{t}}=150 \mathrm{GeV}$. The figures also show the SM predictions for $m_{\mathrm{t}}=175 \mathrm{GeV}$ (dotted lines). 

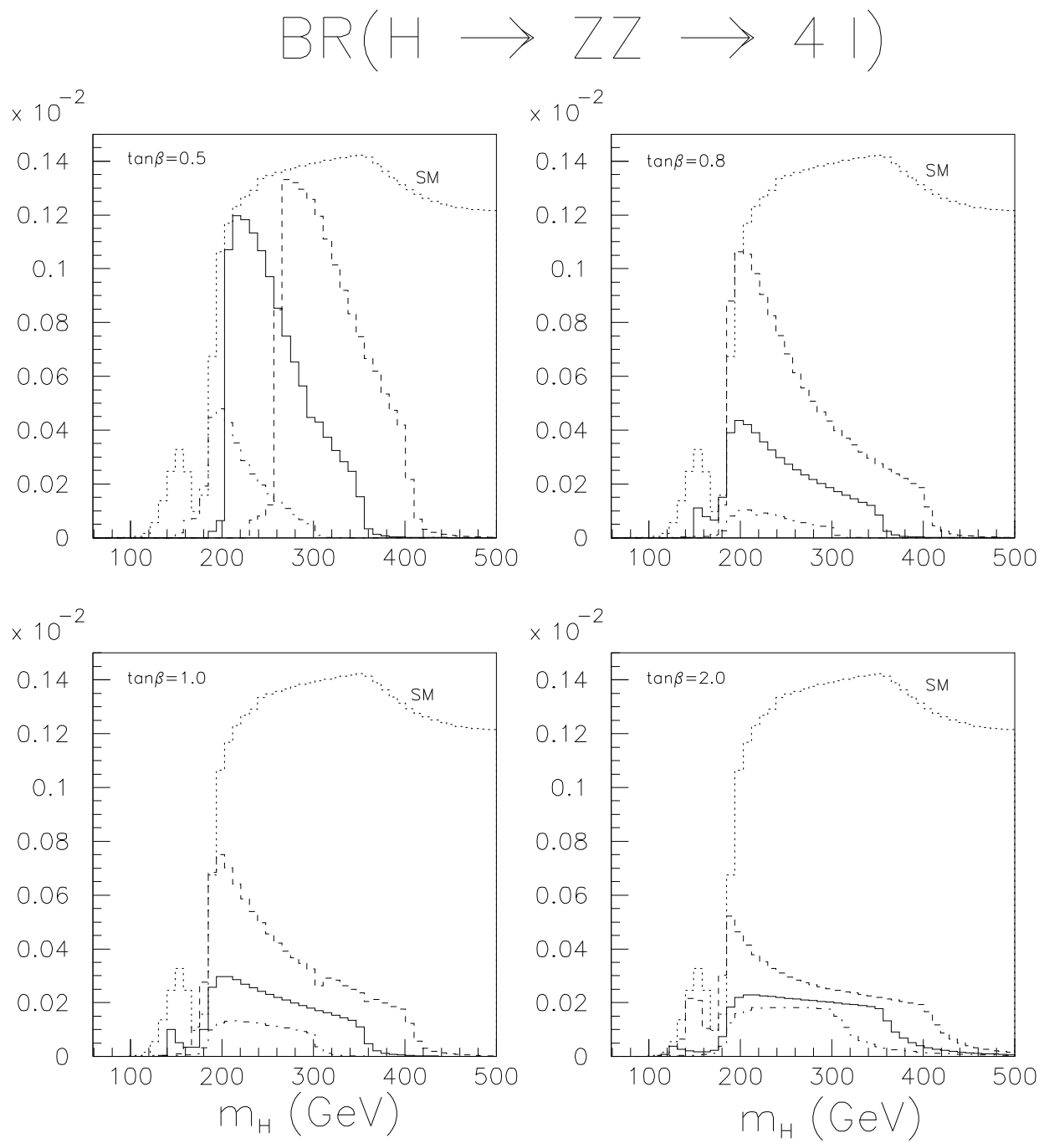

Figure 13: $\mathrm{H} \rightarrow \mathrm{ZZ}^{(\star)} \rightarrow 4 \ell$ branching ratio as a function of $m_{\mathrm{H}}$ for low values of $\tan \beta$. The solid line is for $m_{\mathrm{t}}=175 \mathrm{GeV}$, the dashed one for $m_{\mathrm{t}}=200 \mathrm{GeV}$ and the dot-dashed one for $m_{\mathrm{t}}=150 \mathrm{GeV}$. The figures also show the $S M$ predictions $m_{\mathrm{t}}=175 \mathrm{GeV}$ (dotted lines). 

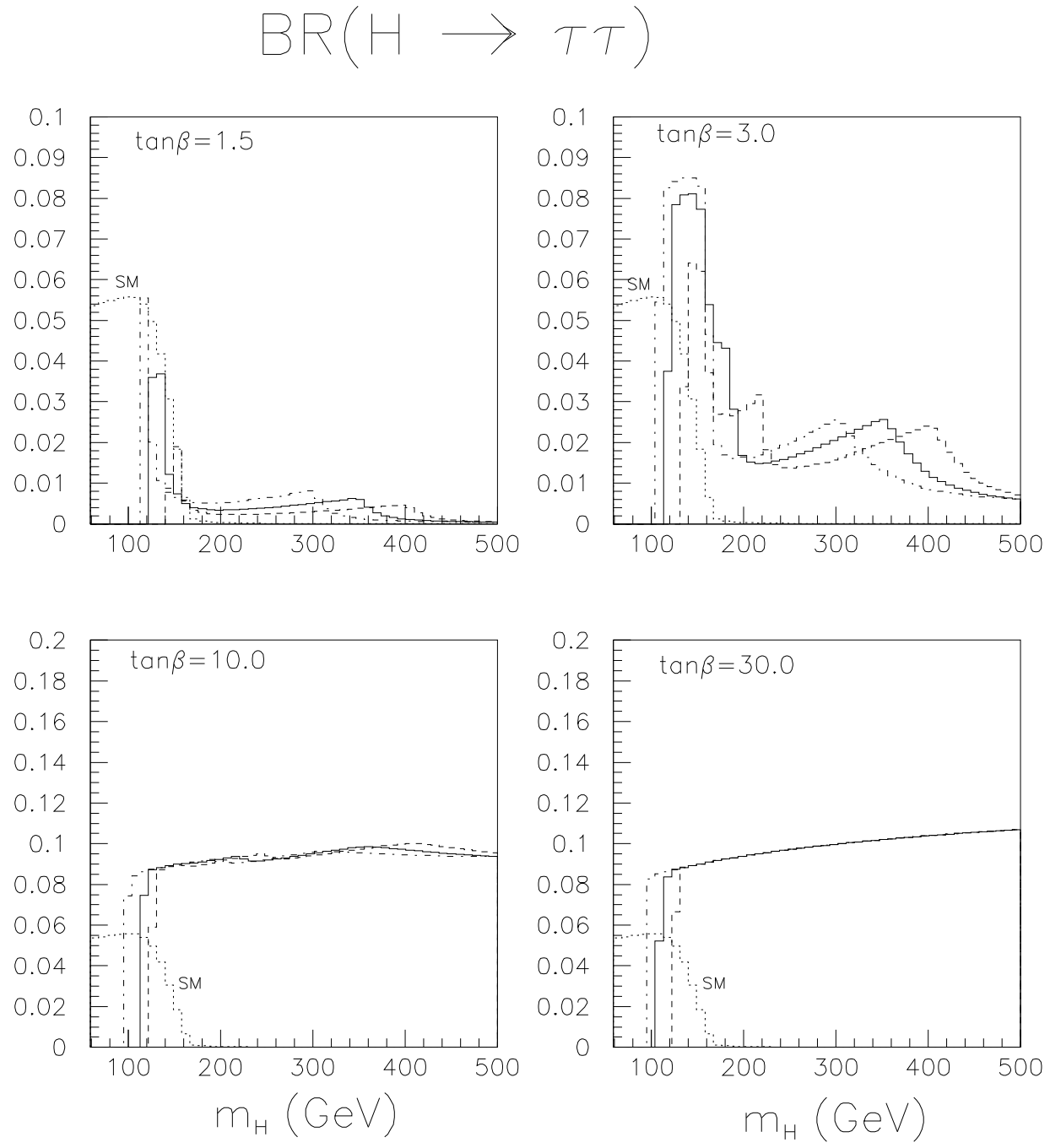

Figure 14: $\quad \mathrm{H} \rightarrow \tau \tau$ branching ratio as a function of $m_{\mathrm{H}}$ for four values of $\tan \beta$. The solid line is for $m_{\mathrm{t}}=175 \mathrm{GeV}$, the dashed one for $m_{\mathrm{t}}=200 \mathrm{GeV}$ and the dot-dashed one for $m_{\mathrm{t}}=150 \mathrm{GeV}$. The figures also show the SM predictions (dotted lines). 

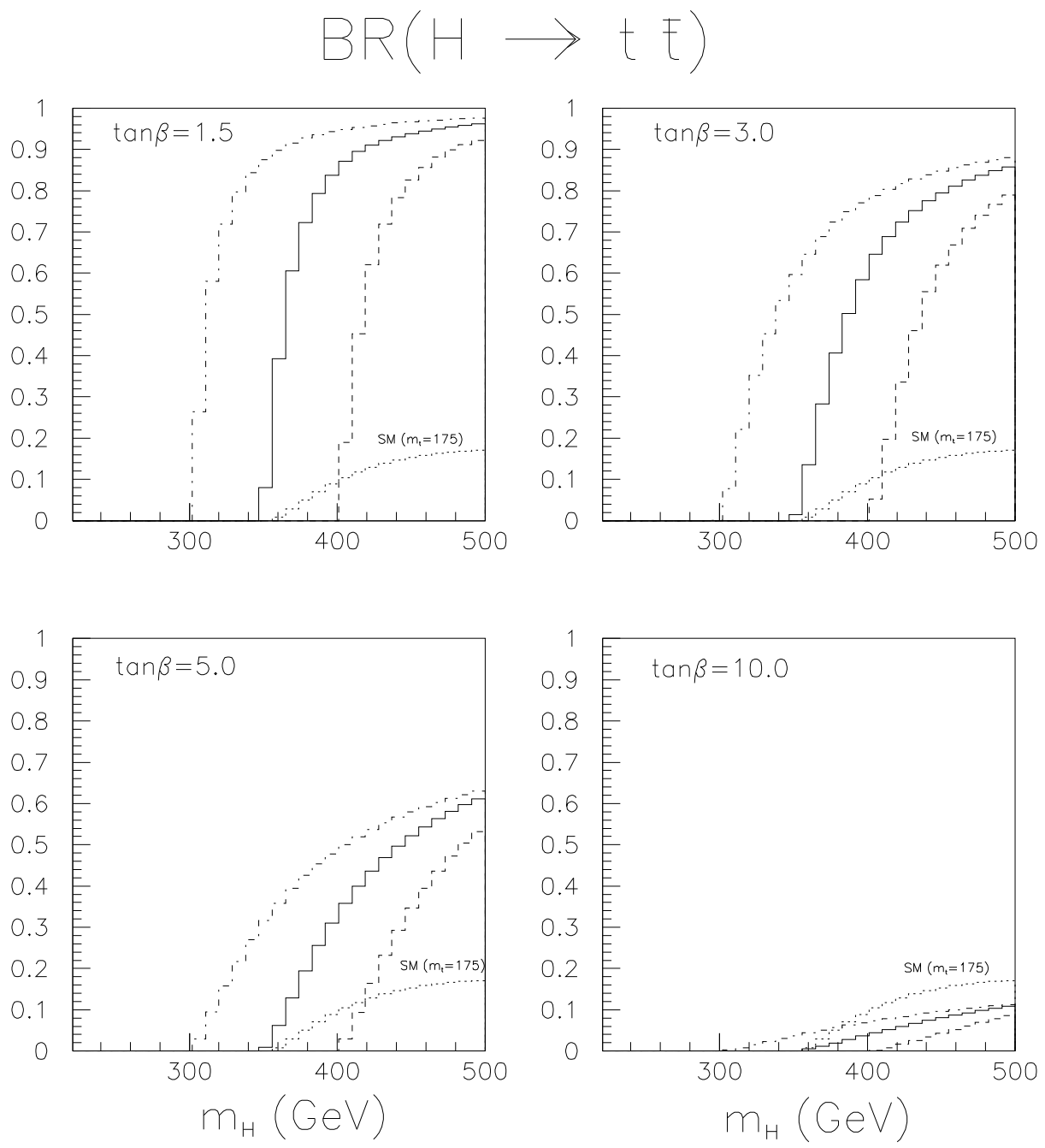

Figure 15: $\mathrm{H} \rightarrow \mathrm{t} \overline{\mathrm{t}}$ branching ratio as a function of $m_{\mathrm{H}}$ for four values of $\tan \beta$. The solid line is for $m_{\mathrm{t}}=175 \mathrm{GeV}$, the dashed one for $m_{\mathrm{t}}=200 \mathrm{GeV}$ and the dot-dashed one for $m_{\mathrm{t}}=150 \mathrm{GeV}$. The figures also show the SM predictions for $m_{\mathrm{t}}=175 \mathrm{GeV}$ (dotted lines). 

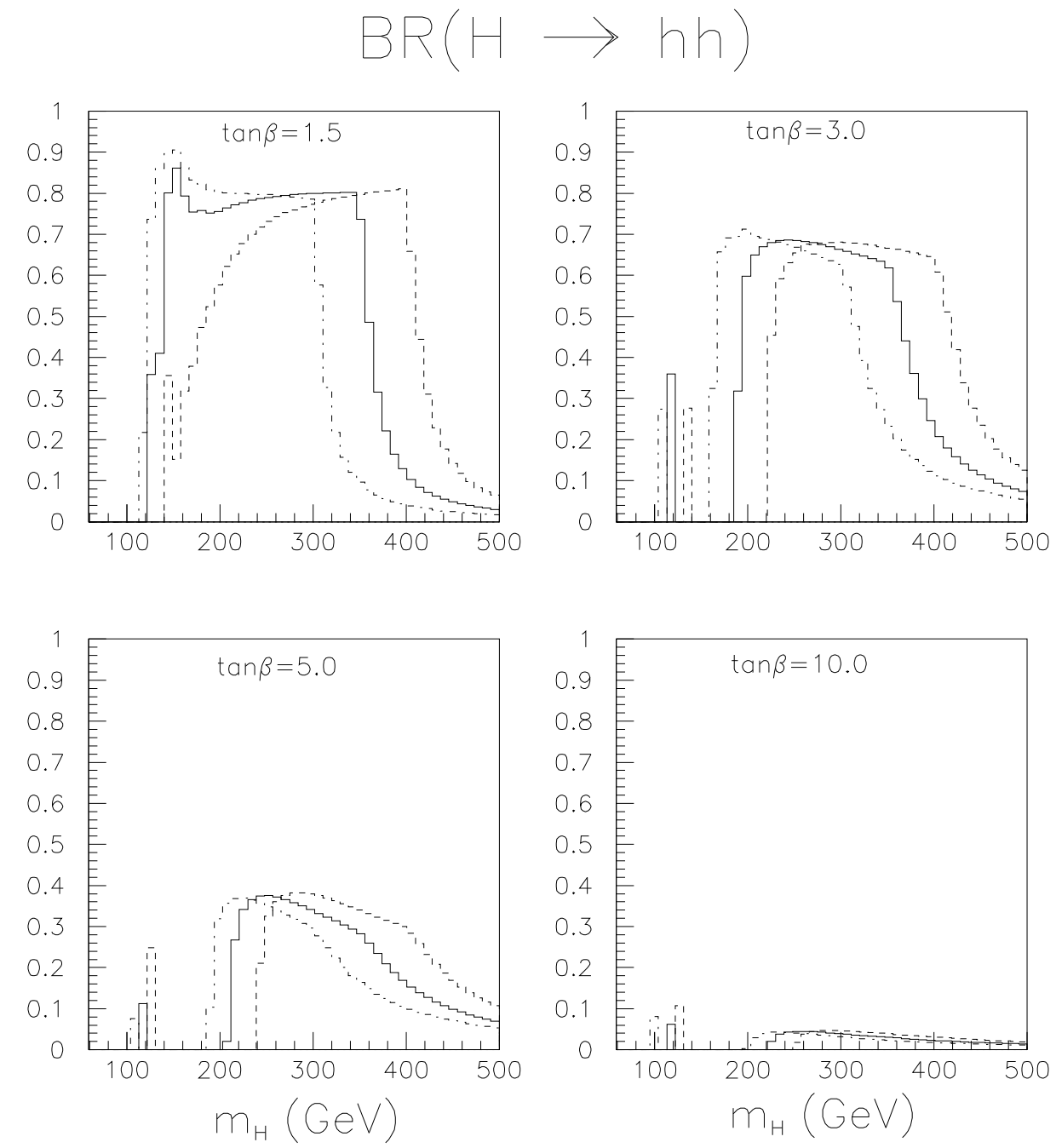

Figure 16: $\mathrm{H} \rightarrow$ hh branching ratio as a function of $m_{\mathrm{H}}$ for four values of $\tan \beta$. The solid line is for $m_{\mathrm{t}}=175 \mathrm{GeV}$, the dashed one for $m_{\mathrm{t}}=200 \mathrm{GeV}$ and the dot-dashed one for $m_{\mathrm{t}}=150 \mathrm{GeV}$. 


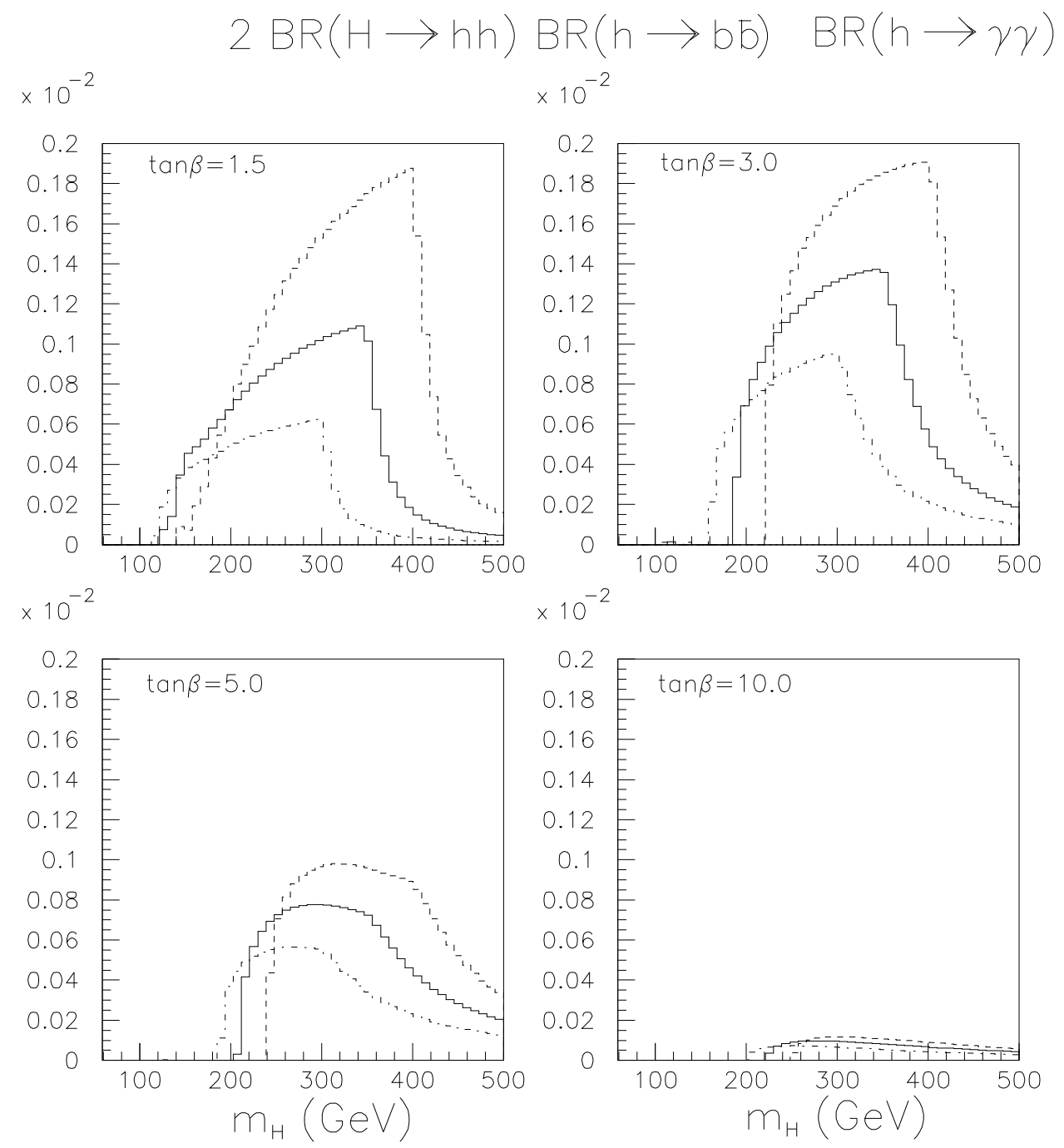

Figure 17: $\mathrm{H} \rightarrow \mathrm{hh} \rightarrow \mathrm{b} \overline{\mathrm{b}} \gamma \gamma$ branching ratio as a function of $m_{\mathrm{H}}$ for four values of $\tan \beta$. The solid line is for $m_{\mathrm{t}}=175 \mathrm{GeV}$, the dashed one for $m_{\mathrm{t}}=200 \mathrm{GeV}$ and the dot-dashed one for $m_{\mathrm{t}}=150 \mathrm{GeV}$. 

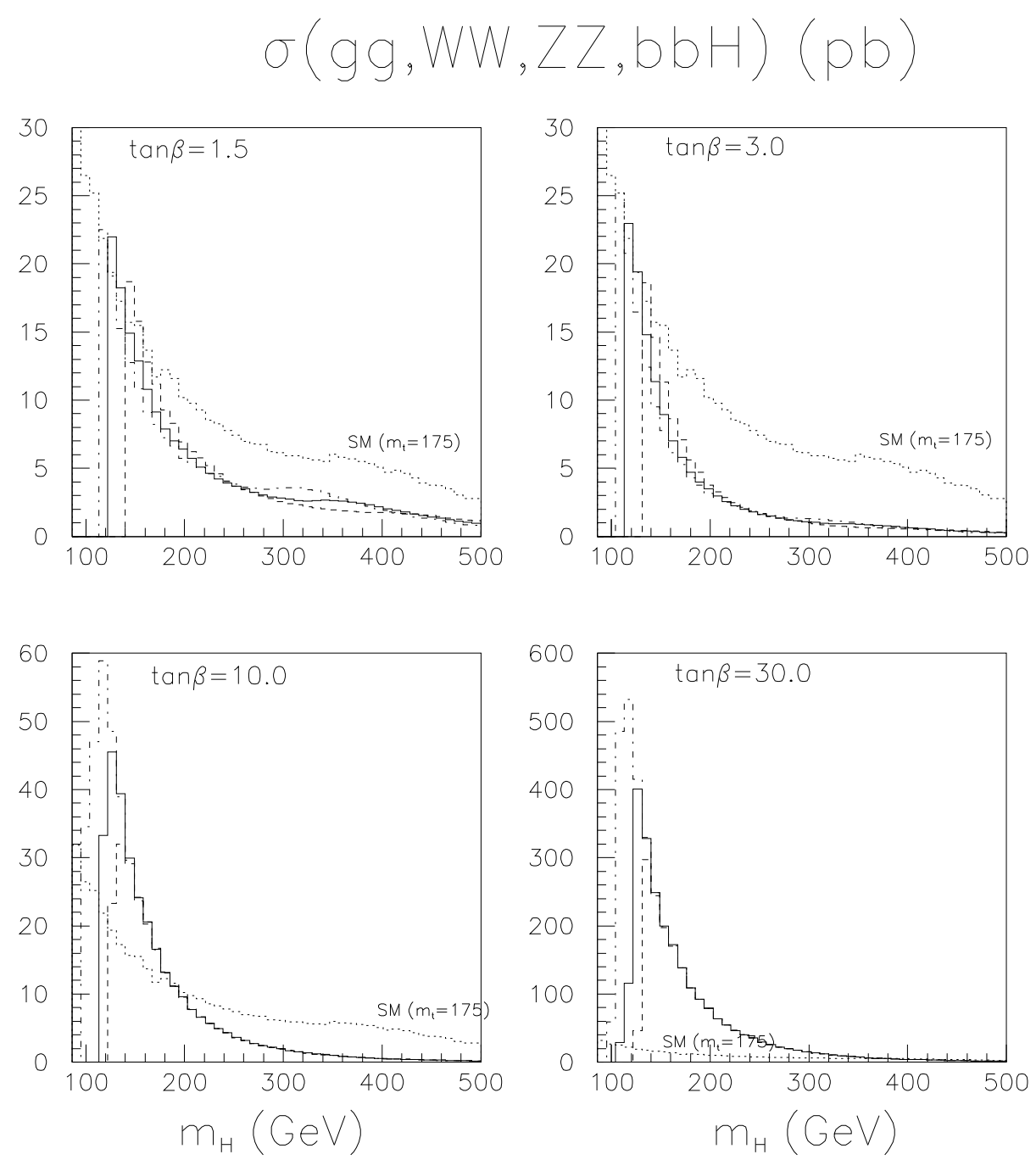

Figure 18: Production cross-section for an $H$-boson $(g g \rightarrow H, q q \rightarrow q q H$, $\mathrm{b} \overline{\mathrm{b}} \mathrm{H})$ as a function of $m_{\mathrm{H}}$ for four values of $\tan \beta$. The solid line is for $m_{\mathrm{t}}=175 \mathrm{GeV}$, the dashed one for $m_{\mathrm{t}}=200 \mathrm{GeV}$ and the dot-dashed one for $m_{\mathrm{t}}=150 \mathrm{GeV}$. The figures also show the SM predictions for $m_{\mathrm{t}}=175 \mathrm{GeV}$ (dotted lines). 


\section{$\sigma(g g, W W, Z Z, b b H)(p b)$}
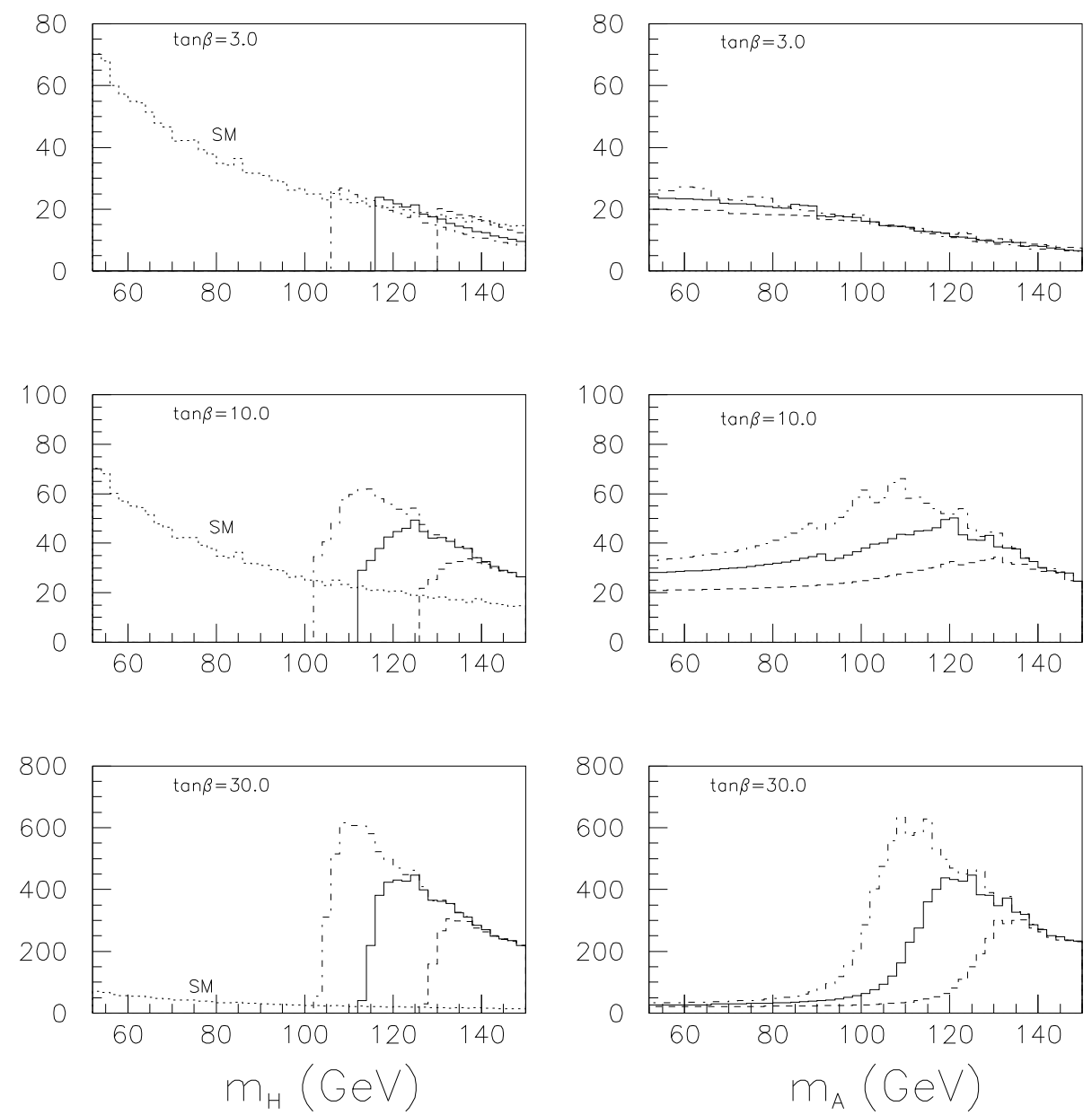

Figure 19: Production cross-section for an $H$-boson $(g g \rightarrow H, q q \rightarrow q q H$, $\mathrm{b} \overline{\mathrm{b} H}$ ) as a function of $m_{\mathrm{H}}$ (left) and as a function of $m_{\mathrm{A}}$ (right) for three values of $\tan \beta$. The solid line is for $m_{\mathrm{t}}=175 \mathrm{GeV}$, the dashed one for $m_{\mathrm{t}}=200 \mathrm{GeV}$ and the dot-dashed one for $m_{\mathrm{t}}=150 \mathrm{GeV}$. The figures also show the SM predictions for $m_{\mathrm{t}}=175 \mathrm{GeV}$ (dotted lines). 

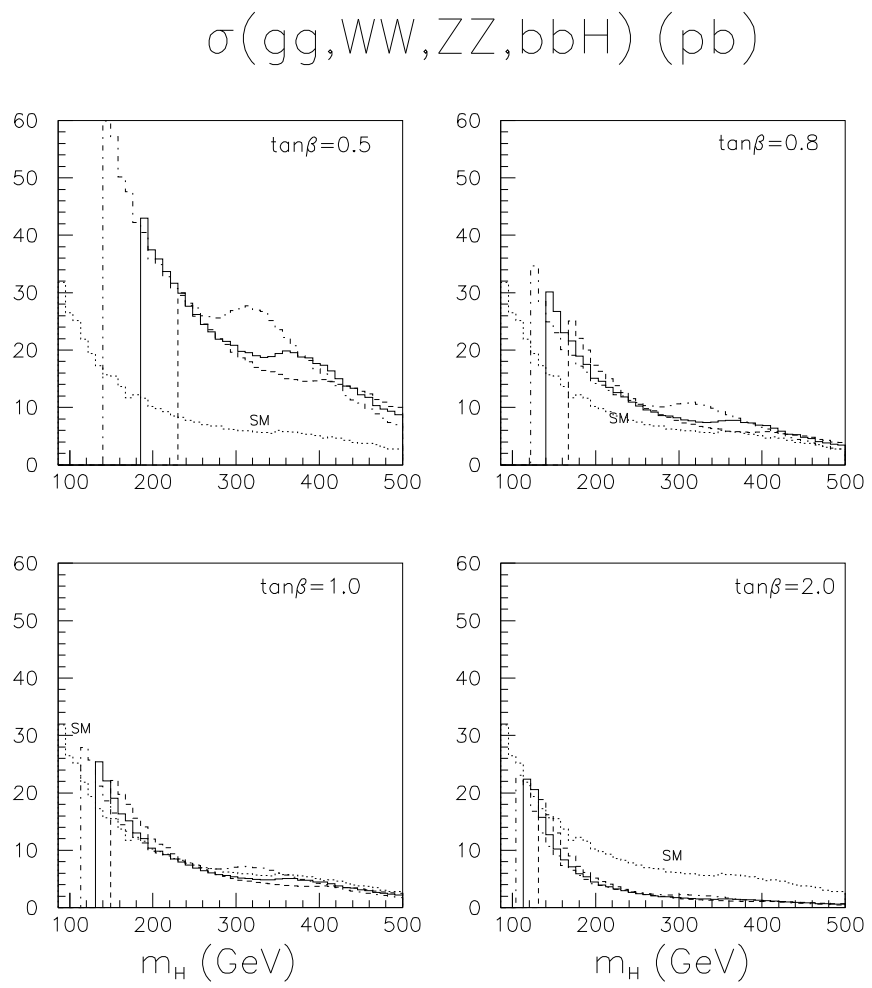

Figure 20: Production cross-section for an $H$-boson $(g g \rightarrow H, q q \rightarrow q q H$, $\mathrm{b} \overline{\mathrm{b}} \mathrm{H})$ as a function of $m_{\mathrm{H}}$ for low values of $\tan \beta$. The solid line is for $m_{\mathrm{t}}=175 \mathrm{GeV}$, the dashed one for $m_{\mathrm{t}}=200 \mathrm{GeV}$ and the dot-dashed one for $m_{\mathrm{t}}=150 \mathrm{GeV}$. The figures also show the SM predictions for $m_{\mathrm{t}}=175 \mathrm{GeV}$ (dotted lines).

\subsubsection{Expected rates for signatures involving the $\mathrm{H}$-boson}

Table $17(g g \rightarrow H, q q \rightarrow q q H, \mathrm{~b} \overline{\mathrm{b} H})$ shows the expected rates $(\sigma \times B R)$ for $\mathrm{H} \rightarrow \gamma \gamma$ decays as a function of $m_{\mathrm{H}}$, for $m_{\mathrm{t}}=175 \mathrm{GeV}$ and four different values of $\tan \beta$. The corresponding rate for a SM Higgs-boson with $m_{\mathrm{H}}=113 \mathrm{GeV}$ is also shown for comparison. The $\mathrm{H} \rightarrow \gamma \gamma$ channel is only accessible for relatively large values of $\tan \beta$ and over a very narrow range of $m_{\mathrm{H}}$ close to the minimum allowed value (e.g. near $m_{\mathrm{H}}=113 \mathrm{GeV}$ for $m_{\mathrm{t}}=175 \mathrm{GeV}$ ), i.e. close to $m_{\mathrm{A}}=60 \mathrm{GeV}$. The largest rates expected for the MSSM H-boson are comparable to the SM H $\rightarrow \gamma \gamma$ rates, as illustrated in Fig. 21.

Tables 18 and 19 show the expected rates for $\mathrm{H} \rightarrow \mathrm{ZZ}^{(*)} \rightarrow 4 \ell$ decays for a range of values of $\tan \beta$. Except for values of $\tan \beta$ lower than 1 , the expected MSSM rates are strongly suppressed with respect to the SM ones. The very rapid decrease of the $\mathrm{H} \rightarrow \mathrm{ZZ}^{(*)} \rightarrow 4 \ell$ branching ratio as $\tan \beta$ increases cannot be compensated by the rise in the production cross-section due to the $\mathrm{b} \overline{\mathrm{b}} \mathrm{H}$ contribution. 
Table 17: Expected rates $(\sigma \times B R)$ for $\mathrm{H} \rightarrow \gamma \gamma$ decays $(g g \rightarrow H, q q \rightarrow q q H$, $\mathrm{b} \overline{\mathrm{b}} \mathrm{H})$ as a function of $m_{\mathrm{H}}$, for $m_{\mathrm{t}}=175 \mathrm{GeV}$ and four different values of $\tan \beta$. The corresponding rate in the SM case is taken from Table 7 of [3].

\begin{tabular}{|c|c|c|c|c|c|}
\hline$m_{\mathrm{H}}(\mathrm{GeV})$ & \multicolumn{5}{|c|}{$\sigma \times B R(\mathrm{fb})$} \\
\hline & SM Higgs & \multicolumn{4}{|c|}{ MSSM H-boson } \\
\hline & & $\tan \beta=5.0$ & $\tan \beta=10.0$ & $\tan \beta=30.0$ & $\tan \beta=50.0$ \\
\hline 113.0 & 44.7 & - & - & $47.3\left(m_{\mathrm{A}} \sim 57\right)$ & $2.3\left(m_{\mathrm{A}} \sim 50\right)$ \\
\hline 113.1 & & - & - & $16.0\left(m_{\mathrm{A}} \sim 80\right)$ & $53.6\left(m_{\mathrm{A}} \sim 57\right)$ \\
\hline 113.2 & & - & - & $9.3\left(m_{\mathrm{A}} \sim 90\right)$ & $7.1\left(m_{\mathrm{A}} \sim 94\right)$ \\
\hline 113.3 & & - & $2.3\left(m_{\mathrm{A}} \sim 50\right)$ & $6.4\left(m_{\mathrm{A}} \sim 95\right)$ & $4.0\left(m_{\mathrm{A}} \sim 100\right)$ \\
\hline 113.4 & & - & $5.4\left(m_{\mathrm{A}} \sim 56\right)$ & $5.0\left(m_{\mathrm{A}} \sim 98\right)$ & $2.7\left(m_{\mathrm{A}} \sim 105\right)$ \\
\hline 113.5 & & - & $42.4\left(m_{\mathrm{A}} \sim 60\right)$ & $4.2\left(m_{\mathrm{A}} \sim 100\right)$ & $2.2\left(m_{\mathrm{A}} \sim 107\right)$ \\
\hline 113.6 & & - & $37.7\left(m_{\mathrm{A}} \sim 64\right)$ & $3.5\left(m_{\mathrm{A}} \sim 102\right)$ & $1.9\left(m_{\mathrm{A}} \sim 108\right)$ \\
\hline 113.7 & & - & $33.3\left(m_{\mathrm{A}} \sim 67\right)$ & $3.0\left(m_{\mathrm{A}} \sim 103\right)$ & $1.7\left(m_{\mathrm{A}} \sim 109\right)$ \\
\hline 113.8 & & - & $29.2\left(m_{\mathrm{A}} \sim 70\right)$ & $2.7\left(m_{\mathrm{A}} \sim 105\right)$ & $1.4\left(m_{\mathrm{A}} \sim 110\right)$ \\
\hline 114.0 & & - & $23.0\left(m_{\mathrm{A}} \sim 75\right)$ & $2.4\left(m_{\mathrm{A}} \sim 106\right)$ & $1.2\left(m_{\mathrm{A}} \sim 111\right)$ \\
\hline 114.5 & & $2.4\left(m_{\mathrm{A}} \sim 50\right)$ & $14.9\left(m_{\mathrm{A}} \sim 83\right)$ & $1.6\left(m_{\mathrm{A}} \sim 109\right)$ & $0.8\left(m_{\mathrm{A}} \sim 112\right)$ \\
\hline 115.0 & & $9.1\left(m_{\mathrm{A}} \sim 58\right)$ & $11.0\left(m_{\mathrm{A}} \sim 88\right)$ & $1.2\left(m_{\mathrm{A}} \sim 111\right)$ & $0.7\left(m_{\mathrm{A}} \sim 113\right)$ \\
\hline 115.5 & & $34.0\left(m_{\mathrm{A}} \sim 60\right)$ & $9.0\left(m_{\mathrm{A}} \sim 90\right)$ & $0.8\left(m_{\mathrm{A}} \sim 112\right)$ & $0.5\left(m_{\mathrm{A}} \sim 114\right)$ \\
\hline 116.0 & & $28.0\left(m_{\mathrm{A}} \sim 68\right)$ & $7.0\left(m_{\mathrm{A}} \sim 93\right)$ & $0.7\left(m_{\mathrm{A}} \sim 113\right)$ & $0.4\left(m_{\mathrm{A}} \sim 115\right)$ \\
\hline 116.5 & & $23.7\left(m_{\mathrm{A}} \sim 72\right)$ & $4.5\left(m_{\mathrm{A}} \sim 99\right)$ & $0.5\left(m_{\mathrm{A}} \sim 114\right)$ & $0.4\left(m_{\mathrm{A}} \sim 116\right)$ \\
\hline 117.0 & & $20.7\left(m_{\mathrm{A}} \sim 75\right)$ & $3.8\left(m_{\mathrm{A}} \sim 101\right)$ & $0.4\left(m_{\mathrm{A}} \sim 115\right)$ & $0.4\left(m_{\mathrm{A}} \sim 117\right)$ \\
\hline
\end{tabular}
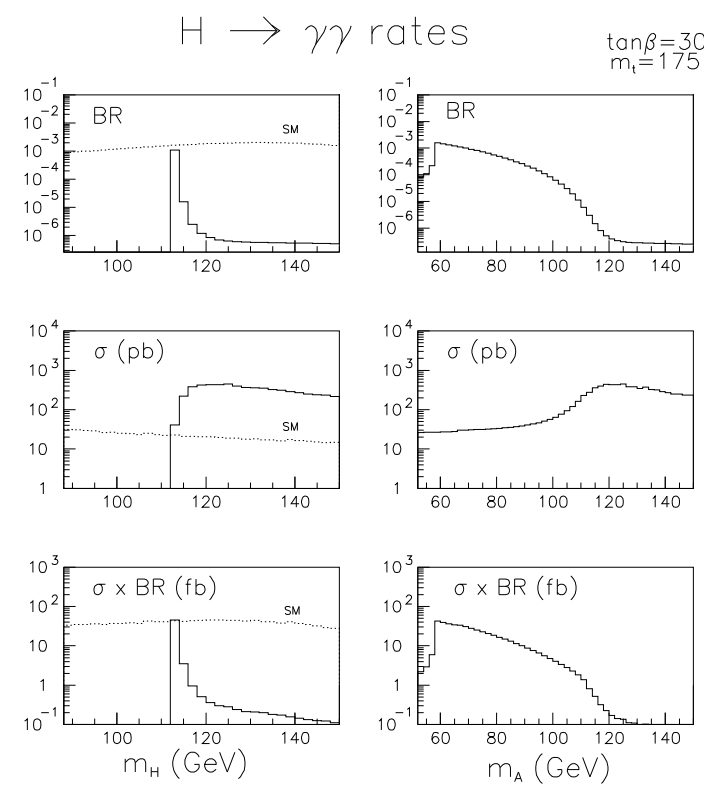

Figure 21: For $\tan \beta=30.0$ and $m_{\mathrm{t}}=175 \mathrm{GeV}$, branching ratio, production cross-section and expected rate for $M S S M \mathrm{H} \rightarrow \gamma \gamma$ decays as a function of $m_{\mathrm{H}}$ (left side) and $m_{\mathrm{A}}$ (right side). 
Table 18: Expected rates $(\sigma \times B R)$ for $\mathrm{H} \rightarrow \mathrm{ZZ}^{(\star)} \rightarrow 4 \ell$ decays $(g g \rightarrow H$, $q q \rightarrow q q H, \mathrm{~b} \overline{\mathrm{b} H})$ as a function of $m_{\mathrm{H}}$, for $m_{\mathrm{t}}=175 \mathrm{GeV}$ and four different values of $\tan \beta$. The corresponding rates in the $S M$ case are taken from Tables 29 and 34 of [3].

\begin{tabular}{|c||c||c|c|c|c|}
\hline \multicolumn{1}{|c||}{$m_{\mathrm{H}}(\mathrm{GeV})$} & \multicolumn{5}{c|}{$\sigma \times B R(\mathrm{fb})$} \\
\hline & SM Higgs & \multicolumn{4}{c|}{ MSSM H-boson } \\
\hline & & $\tan \beta=1.5$ & $\tan \beta=3.0$ & $\tan \beta=5.0$ & $\tan \beta=10.0$ \\
\hline \hline 130.0 & 3.12 & 1.62 & 0.44 & 0.16 & 0.024 \\
150.0 & 5.73 & 0.32 & 0.44 & 0.10 & 0.019 \\
170.0 & 1.44 & 0.26 & 0.35 & 0.13 & 0.027 \\
180.0 & 3.33 & 0.56 & 1.25 & 0.40 & 0.075 \\
200.0 & 12.4 & 1.50 & 0.83 & 0.70 & 0.160 \\
220.0 & 11.9 & 1.22 & 0.57 & 0.33 & 0.108 \\
240.0 & 11.2 & 0.97 & 0.43 & 0.25 & 0.070 \\
260.0 & 10.3 & 0.82 & 0.31 & 0.14 & 0.045 \\
280.0 & 9.60 & 0.68 & 0.24 & 0.10 & 0.027 \\
300.0 & 9.10 & 0.60 & 0.20 & 0.08 & 0.020 \\
320.0 & 8.90 & 0.48 & 0.16 & 0.05 & 0.013 \\
\hline
\end{tabular}

Table 19: Expected rates $(\sigma \times B R)$ for $\mathrm{H} \rightarrow \mathrm{ZZ}^{(\star)} \rightarrow 4 \ell$ decays $(g g \rightarrow H$, $q q \rightarrow q q H, \mathrm{~b} \overline{\mathrm{bH}})$ as a function of $m_{\mathrm{H}}$, for $m_{\mathrm{t}}=175 \mathrm{GeV}$ and low values of $\tan \beta$. The corresponding rates in the SM case are taken from Tables 29 and 34 of [3].

\begin{tabular}{|c||c||c|c|c|c|}
\hline \multicolumn{1}{|c||}{$m_{\mathrm{H}}(\mathrm{GeV})$} & \multicolumn{5}{c|}{$\sigma \times B R(\mathrm{fb})$} \\
\hline & SM Higgs & \multicolumn{4}{c|}{ MSSM H-boson } \\
\hline & & $\tan \beta=0.5$ & $\tan \beta=0.8$ & $\tan \beta=1.0$ & $\tan \beta=2.0$ \\
\hline \hline 130.0 & 3.12 & - & - & 0.10 & $\mathbf{0 . 8 2}$ \\
150.0 & 5.73 & - & 0.10 & 1.31 & $\mathbf{0 . 2 2}$ \\
170.0 & 1.44 & - & 1.41 & 0.54 & 0.16 \\
180.0 & 3.33 & $\mathbf{0 . 9 9}$ & 4.38 & 1.83 & $\mathbf{0 . 5 8}$ \\
200.0 & 12.4 & 5.61 & 6.94 & 3.33 & 1.10 \\
220.0 & 11.9 & 45.5 & 5.36 & 2.60 & $\mathbf{0 . 9 2}$ \\
240.0 & 11.2 & 34.0 & 3.80 & 1.98 & $\mathbf{0 . 6 8}$ \\
260.0 & 10.3 & 22.5 & 2.56 & 1.60 & $\mathbf{0 . 5 0}$ \\
280.0 & 9.60 & 15,2 & 1.97 & 1.23 & 0.43 \\
300.0 & 9.10 & 10.6 & 1.65 & 1.04 & $\mathbf{0 . 3 8}$ \\
320.0 & 8.90 & 6.6 & 1.20 & 0.85 & 0.33 \\
\hline
\end{tabular}


Table 20: Expected rates $(\sigma \times B R)$ for $\mathrm{H} \rightarrow \tau \tau$ decays $(g g \rightarrow H, q q \rightarrow q q H$, $\mathrm{b} \overline{\mathrm{b}} \mathrm{H})$ as a function of $m_{\mathrm{H}}$, for $m_{\mathrm{t}}=175 \mathrm{GeV}$ and four different values of $\tan \beta$. The corresponding rates in the SM case are also shown.

\begin{tabular}{|c||c||c|c|c|c|}
\hline \multicolumn{1}{|c||}{$m_{\mathrm{H}}(\mathrm{GeV})$} & \multicolumn{5}{c|}{$\sigma \times B R(\mathrm{pb})$} \\
\hline \multicolumn{1}{|c||}{} & SM Higgs & \multicolumn{4}{c|}{ MSSM $H$-boson } \\
\hline \multicolumn{1}{|c||}{} & & $\tan \beta=3.0$ & $\tan \beta=10.0$ & $\tan \beta=30.0$ & $\tan \beta=50.0$ \\
\hline \hline 130.0 & $\mathbf{0 . 7 7}$ & 1.403 & 4.56 & 31.3 & 87.4 \\
150.0 & 0.24 & $\mathbf{0 . 8 0 5}$ & 2.22 & 18.6 & 51.6 \\
200.0 & $\mathbf{0 . 0 0 3}$ & $\mathbf{0 . 0 5 5}$ & $\mathbf{0 . 8 3}$ & 7.14 & 19.7 \\
300.0 & $\mathbf{0 . 0 0 0 3}$ & $\mathbf{0 . 0 2 3}$ & $\mathbf{0 . 1 8}$ & 1.50 & 4.12 \\
400.0 & $\mathbf{0 . 0 0 0 1}$ & $\mathbf{0 . 0 0 7}$ & $\mathbf{0 . 0 5}$ & $\mathbf{0 . 4 2}$ & 1.16 \\
500.0 & $\mathbf{0 . 0 0 0 0 5}$ & $\mathbf{0 . 0 0 2}$ & $\mathbf{0 . 0 2}$ & $\mathbf{0 . 1 5}$ & $\mathbf{0 . 4 3}$ \\
\hline
\end{tabular}

Table 21: Expected rates $(\sigma \times B R)$ for $\mathrm{H} \rightarrow \mu \mu$ decays $(g g \rightarrow H, q q \rightarrow q q H$, $\mathrm{b} \overline{\mathrm{b}} \mathrm{H})$ as a function of $m_{\mathrm{H}}$, for $m_{\mathrm{t}}=175 \mathrm{GeV}$ and four different values of $\tan \beta$. The corresponding rates in the SM case are also shown.

\begin{tabular}{|c||c||c|c|c|c|}
\hline \multicolumn{1}{|c||}{$m_{\mathrm{H}}(\mathrm{GeV})$} & \multicolumn{5}{c|}{$\sigma \times B R(\mathrm{fb})$} \\
\hline \multicolumn{1}{|c||}{} & SM Higgs & \multicolumn{4}{c|}{ MSSM $H$-boson } \\
\hline & & $\tan \beta=\mathbf{3 . 0}$ & $\tan \beta=10.0$ & $\tan \beta=30.0$ & $\tan \beta=50.0$ \\
\hline \hline 130.0 & 2.71 & 2.80 & 12.1 & 133.5 & 302.8 \\
150.0 & 1.77 & 2.76 & 7.70 & 64.6 & 178.6 \\
200.0 & $\mathbf{0 . 0 1}$ & $\mathbf{0 . 1 9}$ & 2.86 & 18.9 & 68.4 \\
300.0 & $\mathbf{0 . 0 0 1}$ & $\mathbf{0 . 0 8}$ & $\mathbf{0 . 6 2}$ & 5.18 & 14.3 \\
400.0 & $\mathbf{0 . 0 0 0 5}$ & $\mathbf{0 . 0 3}$ & $\mathbf{0 . 1 8}$ & 1.47 & 4.10 \\
500.0 & $\mathbf{0 . 0 0 0 0 6}$ & $\mathbf{0 . 0 1}$ & $\mathbf{0 . 0 6}$ & $\mathbf{0 . 5 4}$ & 1.50 \\
\hline
\end{tabular}

Table 22: Expected rates $(\sigma \times B R)$ for $\mathrm{H} \rightarrow \mathrm{t} \overline{\mathrm{t}}$ decays $(g g \rightarrow H, q q \rightarrow q q H$, $\mathrm{b} \overline{\mathrm{b}} \mathrm{H})$ as a function of $m_{\mathrm{H}}$, for $m_{\mathrm{t}}=175 \mathrm{GeV}$ and four different values of $\tan \beta$. The corresponding rates in the SM case are also shown.

\begin{tabular}{|c||c||c|c|c|c|}
\hline \multicolumn{1}{|c||}{$m_{\mathrm{H}}(\mathrm{GeV})$} & \multicolumn{5}{c|}{$\sigma \times B R(\mathrm{pb})$} \\
\hline & SM Higgs & \multicolumn{4}{c|}{ MSSM $H$-boson } \\
\hline & & $\tan \beta=1.5$ & $\tan \beta=3.0$ & $\tan \beta=5.0$ & $\tan \beta=10.0$ \\
\hline \hline 370.0 & 0.10 & 1.90 & 0.31 & 0.03 & 0.01 \\
400.0 & 0.23 & 1.87 & 0.43 & 0.06 & 0.01 \\
450.0 & 0.26 & 1.42 & 0.37 & 0.05 & 0.01 \\
500.0 & 0.21 & 0.90 & 0.27 & 0.04 & 0.01 \\
\hline
\end{tabular}


In contrast, Table 20 shows that the $\mathrm{H} \rightarrow \tau \tau$ channel is much more promising in the MSSM case than in the SM case. Most of the enhancement for the MSSM $H \rightarrow \tau \tau$ rates arises from the very large expected production crosssection for large values of $\tan \beta$ (see Fig. 18). Similar observations apply to the $\mathrm{H} \rightarrow \mu \mu$ channel, for which the expected rates are about a factor 30 lower than for $\mathrm{H} \rightarrow \tau \tau$, as shown in Table 21 .

Table 22 shows the expected rates for the $\mathrm{H} \rightarrow \mathrm{t} \overline{\mathrm{t}}$ channel, which is enhanced with respect to the SM case for low values of $\tan \beta$. However, the rapid increase of the $H \rightarrow b \bar{b}$ branching ratio as $\tan \beta$ increases, strongly suppresses the discovery potential for this channel for values of $\tan \beta$ larger than $\sim 3$.

Finally, Table 23 shows the expected rates for the $\mathrm{H} \rightarrow \mathrm{hh} \rightarrow \mathrm{b} \overline{\mathrm{b}} \gamma \gamma$ channel, which are small but observable for $2 m_{\mathrm{h}}<m_{\mathrm{H}}<2 m_{\mathrm{t}}$ and for values of $\tan \beta$ smaller than $\sim 10$.

Table 23: Expected rates $(\sigma \times B R)$ for $\mathrm{H} \rightarrow \mathrm{hh}$ decays with $\mathrm{h} \rightarrow \mathrm{b} \overline{\mathrm{b}}$ and $\mathrm{h} \rightarrow \gamma \gamma(g g \rightarrow H, q q \rightarrow q q H, \mathrm{~b} \overline{\mathrm{b}} \mathrm{H})$ as a function of $m_{\mathrm{H}}$, for $m_{\mathrm{t}}=175 \mathrm{GeV}$ and four different values of $\tan \beta$.

\begin{tabular}{|c||c|c|c|c|}
\hline \multicolumn{1}{|c||}{$m_{\mathrm{H}}(\mathrm{GeV})$} & \multicolumn{4}{c|}{$\sigma \times B R(\mathrm{fb})$} \\
\hline & \multicolumn{4}{c|}{ MSSM $H$-boson } \\
\hline & $\tan \beta=1.0$ & $\tan \beta=3.0$ & $\tan \beta=5.0$ & $\tan \beta=10.0$ \\
\hline \hline 200.0 & 6.0 & 2.01 & - & - \\
250.0 & 5.0 & 1.60 & 0.94 & 0.28 \\
300.0 & 4.5 & 1.17 & 0.56 & 0.12 \\
350.0 & 1.2 & 1.00 & 0.34 & 0.06 \\
\hline
\end{tabular}




\subsection{A-boson}

The CP-odd neutral Higgs boson, or A-boson, is degenerate in mass with the H-boson over a large fraction of the $\left(m_{\mathrm{A}}, \tan \beta\right)$ plane. Many of the interesting final states ( $\mathrm{t} \overline{\mathrm{t}}, \tau^{+} \tau^{-}, \mu^{+} \mu^{-}$) would therefore be observable for the $\mathrm{H}-$ and A-boson together. The absence of tree-level couplings of the A-boson to gauge-boson pairs has an important impact on the predictions discussed below.

\subsubsection{Branching ratios}

Due to the absence of $Z Z, W W$ and $h h$ decay channels, the $\mathrm{A} \rightarrow \tau \tau$ channel is important even for low values of $\tan \beta$, in contrast to the SM Higgs and to the MSSM $H$-boson. For $100<m_{\mathrm{A}}<400 \mathrm{GeV}$ and for low values of $\tan \beta$, Fig. 22 shows that the $\mathrm{A} \rightarrow \tau \tau$ branching ratio is about a factor 5 higher than the $\mathrm{H} \rightarrow \tau \tau$ branching ratio. Fig. 22 also shows that, as $m_{\mathrm{A}}$ increases, the $\mathrm{A} \rightarrow \tau \tau$ branching ratio sharply decreases for $m_{\mathrm{A}} \sim 200$ and $350 \mathrm{GeV}$ and for low values of $\tan \beta$. These two thresholds correspond to the opening of the $\mathrm{A} \rightarrow \mathrm{Zh}$ and $\mathrm{A} \rightarrow \mathrm{t} \overline{\mathrm{t}}$ channels respectively. For larger values of $\tan \beta$, the dominant decay mode is $\mathrm{A} \rightarrow \mathrm{b} \overline{\mathrm{b}}$, which results in a flat behaviour of the $\mathrm{A} \rightarrow \tau \tau$ branching ratio versus $m_{\mathrm{A}}$. The $\mathrm{A} \rightarrow \mu \mu$ branching ratio shows a very similar behaviour, but the branching ratio is lower by $\left(m_{\mu} / m_{\tau}\right)^{2}$.

As shown in Fig. 23, the $A \rightarrow t \bar{t}$ channel is the dominant one for low values of $\tan \beta$ and for $m_{\mathrm{A}}>2 m_{\mathrm{t}}$. As $\tan \beta$ increases, the $\mathrm{A} \rightarrow \mathrm{t} \overline{\mathrm{t}}$ channel competes with the $\mathrm{A} \rightarrow \mathrm{b} \overline{\mathrm{b}}$ decay channel, and its branching ratio is reduced to less than $20 \%$ for $\tan \beta=10$, where it is close to the SM one.

Over the kinematically allowed region, where $m_{\mathrm{Z}}+m_{\mathrm{h}}<m_{\mathrm{A}}<2 m_{\mathrm{t}}$, Fig. 24 shows that the $\mathrm{A} \rightarrow \mathrm{Zh}$ channel becomes dominant for low values of $\tan \beta$. The shape of the $\mathrm{A} \rightarrow \mathrm{Zh}$ branching ratio is not significantly affected when requiring $h \rightarrow b \bar{b}$ and $Z \rightarrow \ell$ decays in the final state (see Fig. 25). As for the $\mathrm{A} \rightarrow \mathrm{t} \overline{\mathrm{t}}$ channel, the $\mathrm{A} \rightarrow \mathrm{Zh}$ branching ratio decreases rapidly as $\tan \beta$ increases, because the $\mathrm{A} \rightarrow \mathrm{b} \overline{\mathrm{b}}$ decays become dominant. 

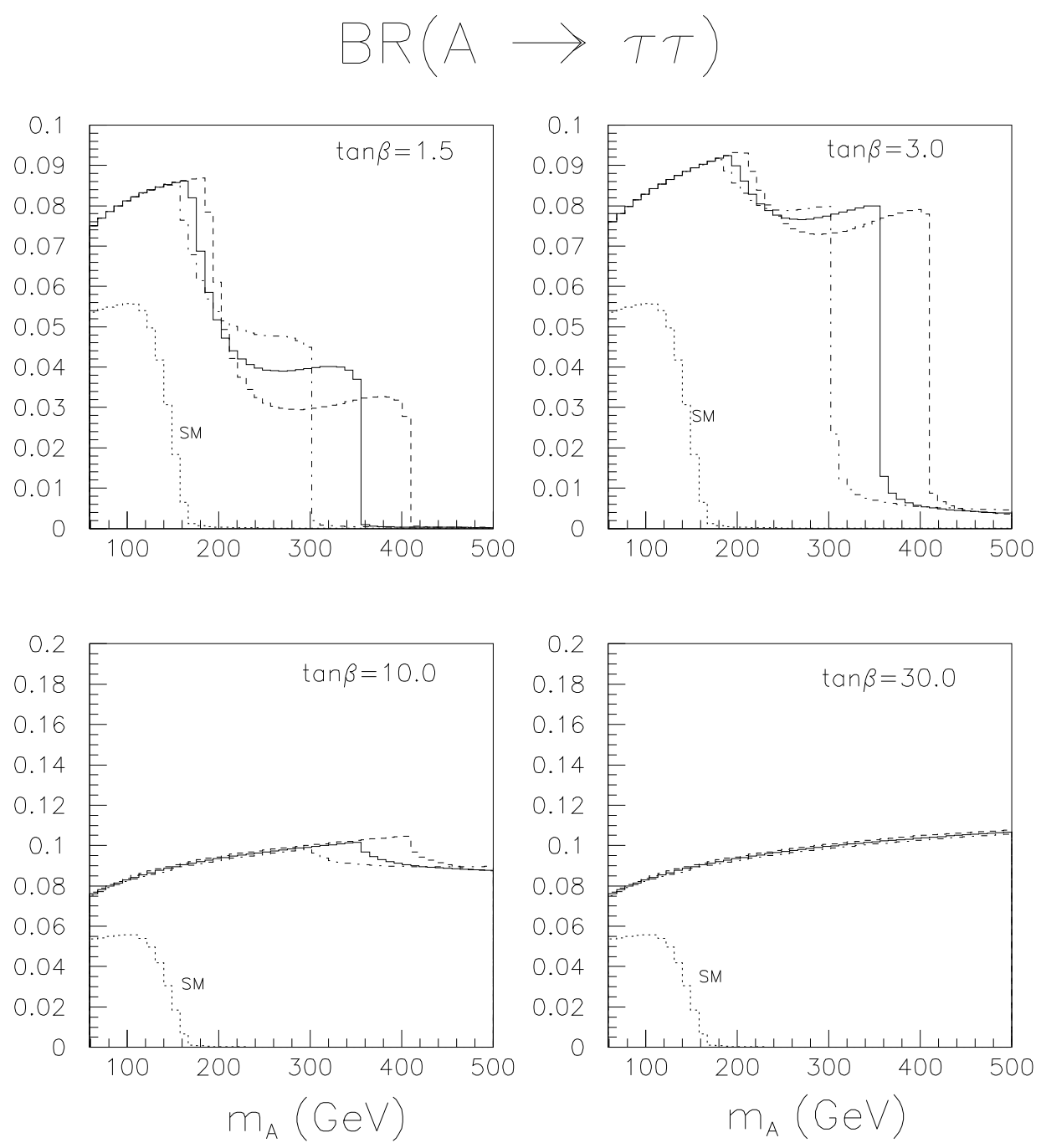

Figure 22: $\quad \mathrm{A} \rightarrow \tau \tau$ branching ratio as a function of $m_{\mathrm{A}}$ for four values of $\tan \beta$. The solid line is for $m_{\mathrm{t}}=175 \mathrm{GeV}$, the dashed one for $m_{\mathrm{t}}=200 \mathrm{GeV}$ and the dot-dashed one for $m_{\mathrm{t}}=150 \mathrm{GeV}$. The figures also show the SM predictions (dotted lines). 

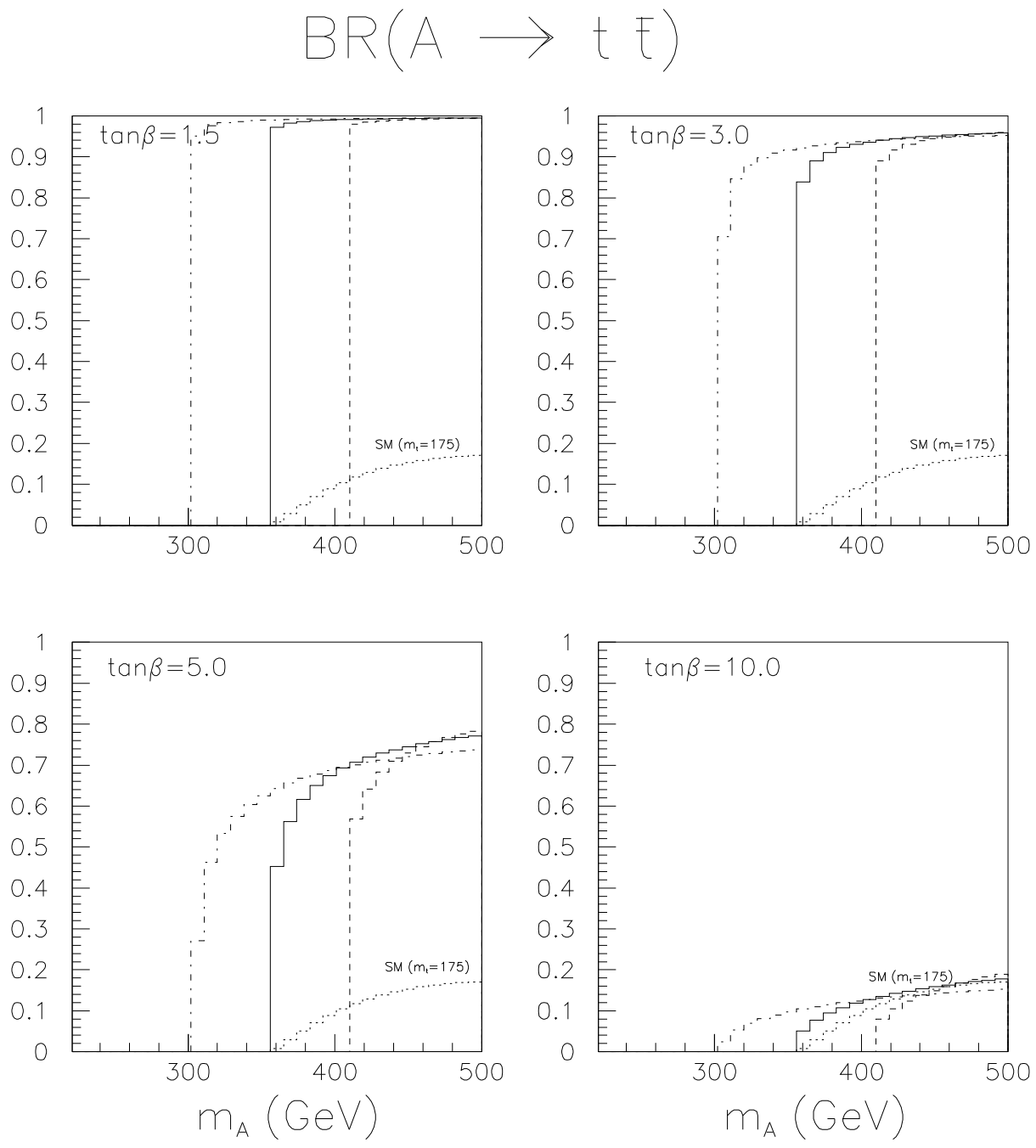

Figure 23: $\mathrm{A} \rightarrow \mathrm{t} \overline{\mathrm{t}}$ branching ratio as a function of $m_{\mathrm{A}}$ for four values of $\tan \beta$. The solid line is for $m_{\mathrm{t}}=175 \mathrm{GeV}$, the dashed one for $m_{\mathrm{t}}=200 \mathrm{GeV}$ and the dot-dashed one for $m_{\mathrm{t}}=150 \mathrm{GeV}$. The figures also show the SM predictions (dotted lines). 

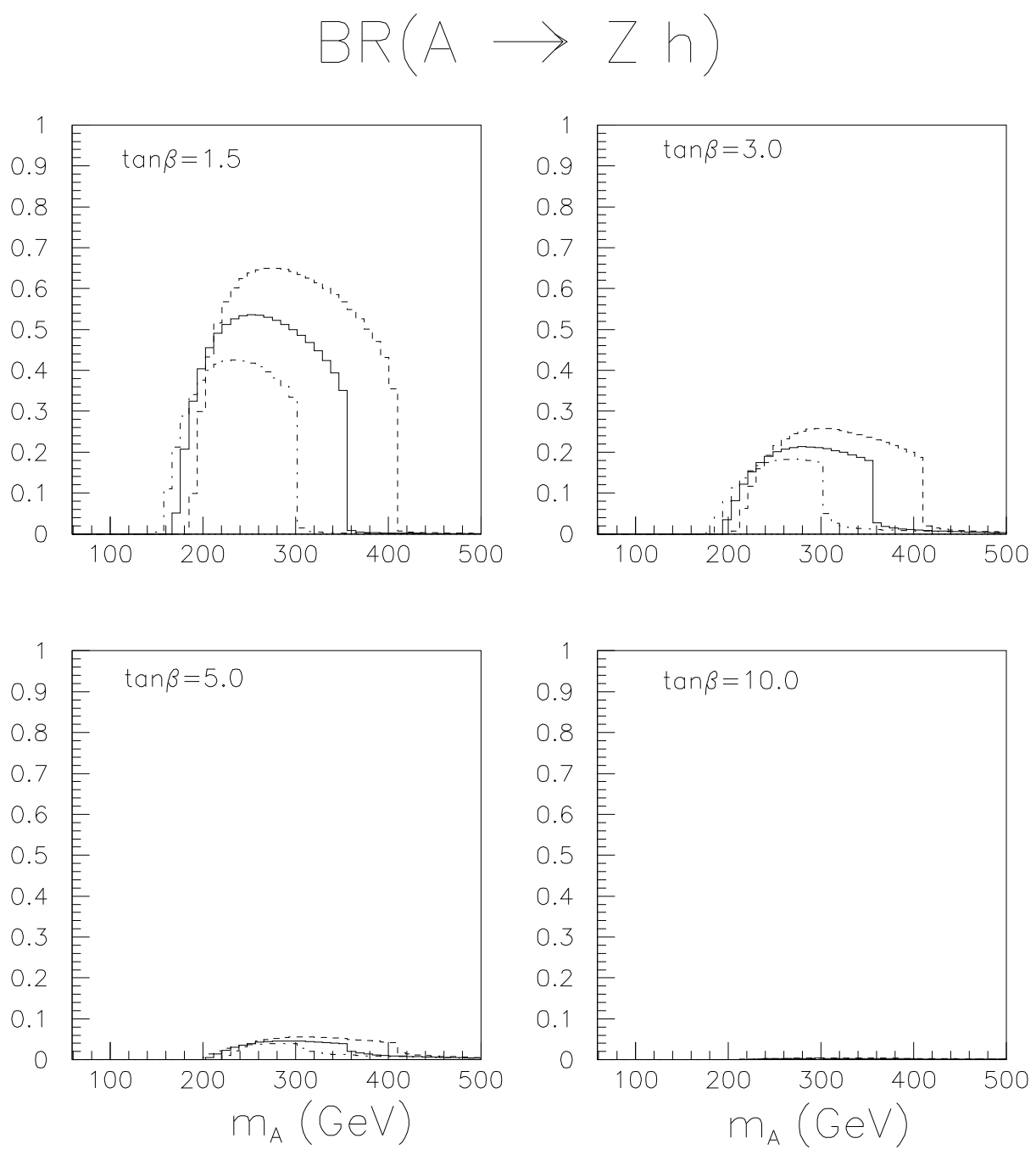

Figure 24: $\mathrm{A} \rightarrow \mathrm{Zh}$ branching ratio as a function of $m_{\mathrm{A}}$ for four values of $\tan \beta$. The solid line is for $m_{\mathrm{t}}=175 \mathrm{GeV}$, the dashed one for $m_{\mathrm{t}}=200 \mathrm{GeV}$ and the dot-dashed one for $m_{\mathrm{t}}=150 \mathrm{GeV}$. 

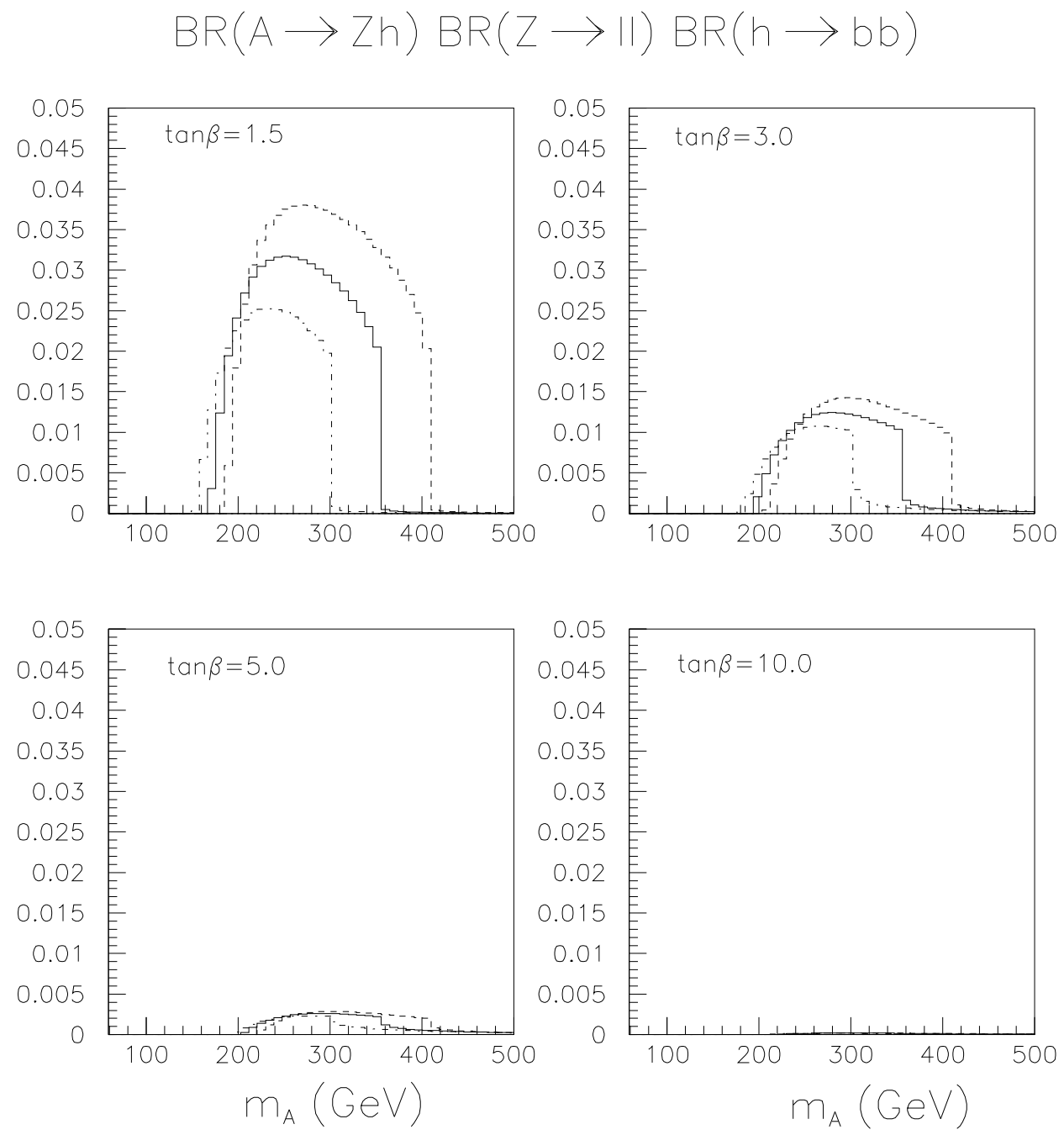

Figure 25: Branching ratio for $\mathrm{A} \rightarrow \mathrm{Zh}$, followed by $\mathrm{h} \rightarrow \mathrm{b} \overline{\mathrm{b}}$ and $\mathrm{Z} \rightarrow \ell \ell$ decays, as a function of $m_{\mathrm{A}}$ and for four values of $\tan \beta$. The solid line is for $m_{\mathrm{t}}=175 \mathrm{GeV}$, the dashed one for $m_{\mathrm{t}}=200 \mathrm{GeV}$ and the dot-dashed one for $m_{\mathrm{t}}=150 \mathrm{GeV}$. 


\subsubsection{Production cross-sections}

Due to the absence of WWA and ZZA couplings, the A-boson is produced only through the $g g$ fusion and $b \bar{b} A$ subprocesses. As shown in Fig. 26, the summed production cross-section is larger than the corresponding SM one in most cases. This effect is most visible for values of $\tan \beta$ larger than $\sim 10$, where the $\mathrm{b} \overline{\mathrm{b}} \mathrm{A}$ subprocess is dominant. For lower values of $\tan \beta$, the $\mathrm{b} \overline{\mathrm{b}} \mathrm{A}$ contribution disappears rapidly and the production cross-section decreases rapidly to values well below the SM Higgs cross-sections. The dependence on $m_{\mathrm{t}}$ is weak, but for the lowest values of $\tan \beta$, the $g g$ cross-section is enhanced and clear peaks in the production cross-section appear around $2 m_{t}$.
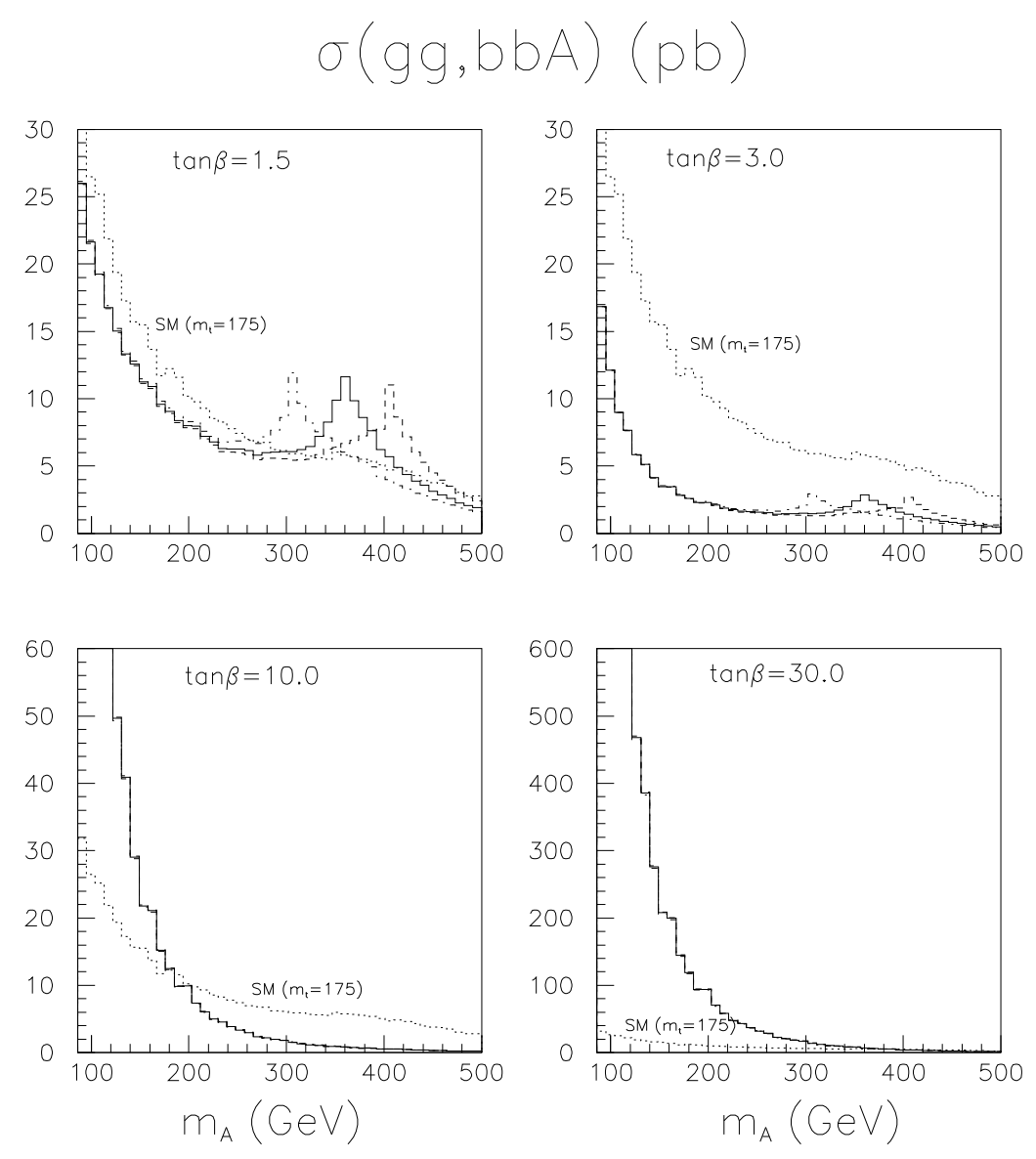

Figure 26: Production cross-section for an A-boson $(g g \rightarrow A, \bar{b} \overline{\mathrm{b}} \mathrm{A})$ as a function of $m_{\mathrm{A}}$ for four values of $\tan \beta$. The solid line is for $m_{\mathrm{t}}=175 \mathrm{GeV}$, the dashed one for $m_{\mathrm{t}}=200 \mathrm{GeV}$ and the dot-dashed one for $m_{\mathrm{t}}=150 \mathrm{GeV}$. The figures also show the SM predictions for $m_{\mathrm{t}}=175 \mathrm{GeV}$ (dotted lines). 


\subsubsection{Expected rates for signatures involving the A-boson}

Tables $24(\mathrm{~A} \rightarrow \tau \tau$ decays $), 25(\mathrm{~A} \rightarrow \mu \mu$ decays $)$ and $26(\mathrm{~A} \rightarrow \mathrm{t} \overline{\mathrm{t}}$ decays $)$ show that the expected rates have the same properties as in the case of the $\mathrm{H}$-boson. The only noticeable difference is for low values of $\tan \beta$, where the larger production cross-section, and to a lesser extent the larger branching ratios, result in larger expected rates.

The rates expected in the $\mathrm{A} \rightarrow \mathrm{Zh}$ channel, with $\mathrm{h} \rightarrow \mathrm{b} \overline{\mathrm{b}}$ and $\mathrm{Z} \rightarrow \ell \ell$ decays in the final state, are shown in Table 27.

Table 24: Expected rates $(\sigma \times B R)$ for $\mathrm{A} \rightarrow \tau \tau$ decays $(g g \rightarrow A$, $\mathrm{b} \overline{\mathrm{b}} \mathrm{A})$ as a function of $m_{\mathrm{A}}$, for $m_{\mathrm{t}}=175 \mathrm{GeV}$ and four different values of $\tan \beta$. The corresponding rates in the $S M$ case are also shown.

\begin{tabular}{|c||c||c|c|c|c|}
\hline \multicolumn{1}{|c||}{$m_{\mathrm{A}}(\mathrm{GeV})$} & \multicolumn{5}{c|}{$\sigma \times B R(\mathrm{pb})$} \\
\hline & SM Higgs & \multicolumn{4}{c|}{ MSSM $A$-boson } \\
\hline & & $\tan \beta=1.5$ & $\tan \beta=3.0$ & $\tan \beta=10.0$ & $\tan \beta=30.0$ \\
\hline \hline 130.0 & $\mathbf{0 . 7 7}$ & 1.35 & $\mathbf{0 . 5 3}$ & 3.38 & 32.2 \\
150.0 & 0.24 & 1.05 & $\mathbf{0 . 3 2}$ & 1.97 & 18.8 \\
200.0 & $\mathbf{0 . 0 0 3}$ & $\mathbf{0 . 3 0}$ & $\mathbf{0 . 1 9}$ & $\mathbf{0 . 7 4}$ & 5.43 \\
300.0 & $\mathbf{0 . 0 0 0 3}$ & $\mathbf{0 . 2 7}$ & $\mathbf{0 . 1 2}$ & $\mathbf{0 . 1 5}$ & 1.47 \\
400.0 & $\mathbf{0 . 0 0 0 1}$ & $\mathbf{0 . 0 0 2}$ & $\mathbf{0 . 0 1}$ & $\mathbf{0 . 0 5}$ & $\mathbf{0 . 4 2}$ \\
$\mathbf{5 0 0 . 0}$ & $\mathbf{0 . 0 0 0 0 5}$ & $\mathbf{0 . 0 0 0 5}$ & $\mathbf{0 . 0 0 1}$ & $\mathbf{0 . 0 2}$ & $\mathbf{0 . 1 5}$ \\
\hline
\end{tabular}

Table 25: Expected rates $(\sigma \times B R)$ for $\mathrm{A} \rightarrow \mu \mu$ decays $(g g \rightarrow A$, $\mathrm{b} \overline{\mathrm{b}} \mathrm{A})$ as a function of $m_{\mathrm{A}}$, for $m_{\mathrm{t}}=175 \mathrm{GeV}$ and four different values of $\tan \beta$. The corresponding rates in the SM case are also shown.

\begin{tabular}{|c||c||c|c|c|c|}
\hline \multicolumn{1}{|c||}{$m_{\mathrm{A}}(\mathrm{GeV})$} & \multicolumn{5}{c|}{$\sigma \times B R(\mathrm{fb})$} \\
\hline & SM Higgs & \multicolumn{4}{c|}{ MSSM $A$-boson } \\
\hline & & $\tan \beta=1.5$ & $\tan \beta=3.0$ & $\tan \beta=10.0$ & $\tan \beta=30.0$ \\
\hline \hline 130.0 & 2.71 & 4.5 & $\mathbf{0 . 4 4}$ & 11.7 & 111. \\
150.0 & 1.77 & 4.0 & $\mathbf{0 . 3 2}$ & 6.81 & 65.0 \\
200.0 & 0.01 & $\mathbf{0 . 4 1}$ & $\mathbf{0 . 1 9}$ & 2.57 & 24.6 \\
300.0 & 0.001 & 0.25 & $\mathbf{0 . 1 2}$ & $\mathbf{0 . 5 3}$ & 5.08 \\
400.0 & $\mathbf{0 . 0 0 0 5}$ & $\mathbf{0 . 0 0 0 2}$ & $\mathbf{0 . 0 0 9}$ & $\mathbf{0 . 1 7}$ & 1.44 \\
500.0 & $\mathbf{0 . 0 0 0 0 6}$ & $\mathbf{0 . 0 0 0 0 5}$ & $\mathbf{0 . 0 0 3}$ & $\mathbf{0 . 0 6}$ & $\mathbf{0 . 5 4}$ \\
\hline
\end{tabular}


Table 26: Expected rates $(\sigma \times B R)$ for $\mathrm{A} \rightarrow \mathrm{t} \overline{\mathrm{t}}$ decays $(g g \rightarrow A, \mathrm{~b} \overline{\mathrm{b}} \mathrm{A})$ as a function of $m_{\mathrm{A}}$, for $m_{\mathrm{t}}=175 \mathrm{GeV}$ and four different values of $\tan \beta$. The corresponding rates in the $S M$ case are also shown.

\begin{tabular}{|c||c||c|c|c|c|}
\hline \multicolumn{1}{|c||}{$m_{\mathrm{A}}(\mathrm{GeV})$} & \multicolumn{5}{c|}{$\sigma \times B R(\mathrm{pb})$} \\
\hline & SM Higgs & \multicolumn{4}{c|}{ MSSM $A$-boson } \\
\hline & & $\tan \beta=1.5$ & $\tan \beta=3.0$ & $\tan \beta=5.0$ & $\tan \beta=10.0$ \\
\hline \hline 370.0 & 0.10 & 9.90 & 2.16 & 0.55 & 0.03 \\
400.0 & 0.23 & 6.53 & 1.51 & 0.45 & 0.03 \\
450.0 & 0.26 & 3.38 & 0.80 & 0.27 & 0.02 \\
500.0 & 0.21 & 2.00 & 0.25 & 0.15 & 0.02 \\
\hline
\end{tabular}

Table 27: Expected rates $(\sigma \times B R)$ for $\mathrm{A} \rightarrow \mathrm{Zh}$ decays $(g g \rightarrow A, \mathrm{~b} \overline{\mathrm{b}} \mathrm{A})$, with $\mathrm{Z} \rightarrow \ell \ell$ and $\mathrm{h} \rightarrow \mathrm{b} \overline{\mathrm{b}}$ decays, as a function of $m_{\mathrm{A}}$, for $m_{\mathrm{t}}=175 \mathrm{GeV}$ and four different values of $\tan \beta$.

\begin{tabular}{|c||c||c|c|c|}
\hline \multicolumn{1}{|c||}{$m_{\mathrm{A}}(\mathrm{GeV})$} & \multicolumn{4}{c|}{$\sigma \times B R(\mathrm{fb})$} \\
\hline & \multicolumn{4}{c|}{ MSSM $A$-boson } \\
\hline & $\tan \beta=1.5$ & $\tan \beta=3.0$ & $\tan \beta=5.0$ & $\tan \beta=10.0$ \\
\hline \hline 200.0 & 561 & 9 & 0.27 & - \\
250.0 & 472 & 21 & 2.65 & 0.55 \\
300.0 & 341 & 17 & 1.94 & 0.29 \\
350.0 & 231 & 31 & 2.54 & 0.14 \\
\hline
\end{tabular}




\section{$3.4 \quad \mathrm{H}^{ \pm}$-boson}

The charged Higgs boson can be searched for most easily in top-quark decays, i.e. through $g g \rightarrow \mathrm{t} \overline{\mathrm{t}}$ followed by a $\mathrm{t} \rightarrow \mathrm{H}^{+} \mathrm{b}$ decay, for $m_{\mathrm{W}}<m_{\mathrm{H}^{ \pm}}<m_{\mathrm{t}}$. Pair-production of charged Higgs bosons yields too low a cross-section, and charged Higgs- boson production in supersymmetric particle decays is outside the scope of this report.

\subsubsection{Branching ratios}

If the $\mathrm{H}^{+} \rightarrow \mathrm{t} \overline{\mathrm{b}}$ decay is not kinematically allowed, the dominant decay mode for $\tan \beta>1.5$ is $\mathrm{H}^{+} \rightarrow \tau \nu$. Fig. 27 shows the $\mathrm{H}^{+} \rightarrow \tau \nu$ branching ratio as a function of $m_{\mathrm{H}^{+}}$, for various values of $m_{\mathrm{t}}$ and $\tan \beta$. This does not take into account recent calculations [9], which include possible decays of the charged Higgs boson to SUSY particles and show that the $\mathrm{H}^{+} \rightarrow \tau \nu$ branching ratio may in some cases decrease significantly for low values of $\tan \beta$. The $\mathrm{H}^{+} \rightarrow \tau \nu$ branching ratio is close to $100 \%$ and flat as a function of $m_{\mathrm{H}^{+}}$. If $m_{\mathrm{H}^{+}}$becomes larger than $m_{\mathrm{t}}$, then the $\mathrm{H}^{+} \rightarrow \mathrm{t} \overline{\mathrm{b}}$ branching ratio opens up and the $\mathrm{H}^{+} \rightarrow \tau \nu$ branching ratio decreases more or less rapidly depending on $\tan \beta$.
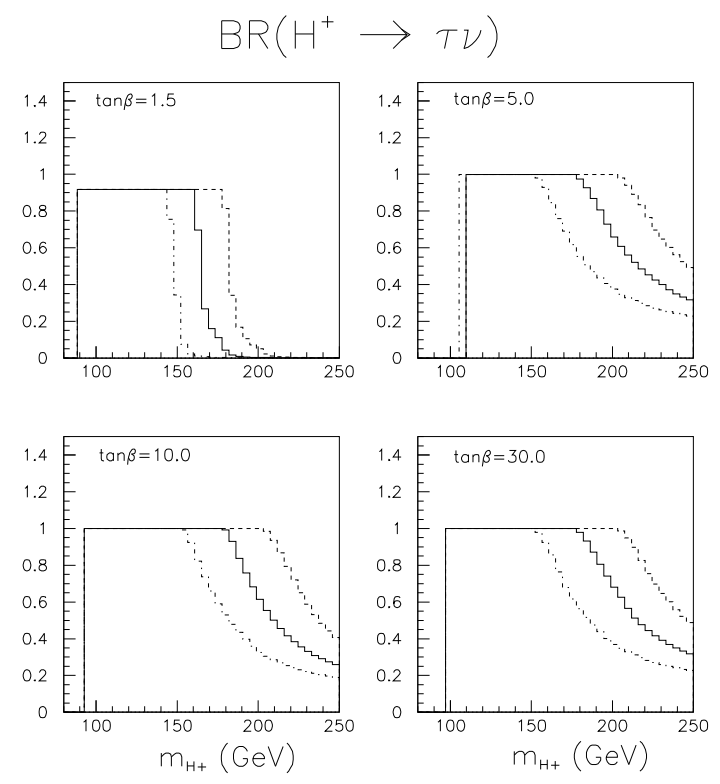

Figure $27: \mathrm{H}^{+} \rightarrow \tau \nu$ branching ratio as a function of $m_{\mathrm{H}^{+}}$for four values of $\tan \beta$. The solid line is for $m_{\mathrm{t}}=175 \mathrm{GeV}$, the dashed one for $m_{\mathrm{t}}=200 \mathrm{GeV}$ and the dot-dashed one for $m_{\mathrm{t}}=150 \mathrm{GeV}$. 


\subsubsection{Production cross-sections}

Charged Higgs production is considered through the $g g \rightarrow t \bar{t}$ process, followed by $\mathrm{t} \rightarrow \mathrm{H}^{+}$b decays, for $m_{\mathrm{H}^{+}}<m_{\mathrm{t}}$. The decay $\mathrm{t} \rightarrow \mathrm{H}^{+} \mathrm{b}$, if kinematically allowed, can then compete with the $t \rightarrow \mathrm{Wb}$ decay. Fig. 28 shows the effective production cross-section for a charged Higgs with positive charge $\sigma_{\mathrm{H}^{+}}=\sigma_{\mathrm{t} \overline{\mathrm{t}}} \times B R\left(\mathrm{t} \rightarrow \mathrm{H}^{+} \mathrm{b}\right)$, as a function of $m_{\mathrm{H}^{+}}$. This cross-section has a minimum around $5<\tan \beta<10$, due to a term proportional to $\left(m_{b}^{2} \tan ^{2} \beta+m_{t}^{2} / \tan ^{2} \beta\right)$ in the $\mathrm{t} \rightarrow \mathrm{H}^{+} \mathrm{b}$ branching ratio. As $m_{\mathrm{H}^{+}}$increases towards $m_{\mathrm{t}}$, the importance of this term decreases as $\left(m_{\mathrm{t}}{ }^{2}-m_{\mathrm{H}^{+}}{ }^{2}\right)$, and the $\mathrm{t} \rightarrow \mathrm{H}^{+} \mathrm{b}$ branching ratio becomes asymptotically independent of $\tan \beta$ for $m_{\mathrm{H}^{+}} \sim m_{\mathrm{t}}$. The effective charged Higgs production cross-section depends

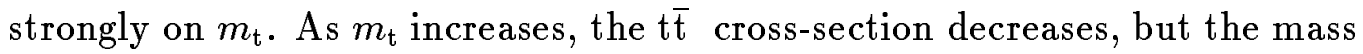
range accessible to $\mathrm{t} \rightarrow \mathrm{H}^{+} \mathrm{b}$ decays increases.

For large values of $\tan \beta$, the $t \rightarrow \mathrm{H}^{+} \mathrm{b}$ branching ratio depends strongly on the value for $m_{b}$ used in the calculation, since one of the main terms used to compute the $\mathrm{t} \rightarrow \mathrm{H}^{+} \mathrm{b}$ branching ratio is proportional to $m_{b}^{2} \tan \beta^{2}$ :

$$
\begin{aligned}
B R\left(t \rightarrow H^{+} b\right)= & \frac{1}{2} \frac{G_{F}^{2}}{32 \pi m_{W}^{2} m_{t}^{3}}\left[\left(m_{H}^{2}+m_{t}^{2}-m_{b}^{2}\right)^{2}+4 m_{H^{+}}^{2} m_{t}^{2}\right] \\
& {\left[\left(m_{t}^{2}-m_{H^{+}}^{2}+m_{b}^{2}\right)\left(m_{b}^{2} \tan ^{2} \beta+m_{t}^{2} / \tan ^{2} \beta\right)+4 m_{b}^{2} m_{t}^{2}\right] }
\end{aligned}
$$

Throughout this report, a running b-quark mass is used ${ }^{5}$ with the evolution defined as $[6]$ :

$$
m_{b}=\bar{m}_{b}\left[\ln \frac{\bar{m}_{b}}{\Lambda_{Q C D}} / \ln \frac{m_{W}}{\Lambda_{Q C D}}\right]^{12 / 23}
$$

for five flavours, $\bar{m}_{b}=4.25 \mathrm{GeV}$, and $\Lambda_{Q C D}=190 \mathrm{MeV}$. The use of the proper running b-quark mass modifies the $\mathrm{t} \rightarrow \mathrm{H}^{+} \mathrm{b}$ branching ratio by as much as $50 \%$ for large values of $\tan \beta$.

\subsubsection{Expected rates for signatures involving the $\mathrm{H}^{ \pm}$boson}

Table 28 presents the expected rates for the $\mathrm{H}^{ \pm} \rightarrow \tau \nu$ final state, defined as $\sigma \times B R=2 \sigma_{\mathrm{t} \overline{\mathrm{t}}} \times B R\left(\mathrm{t} \rightarrow \mathrm{H}^{+} \mathrm{b}\right) \times B R\left(\mathrm{H}^{+} \rightarrow \tau \nu\right)$. This formula neglects the small fraction of events where both top quarks decay to a charged Higgs boson.

\footnotetext{
${ }^{\mathbf{5}}$ For the analysis presented in the TP, this effect was ignored, which led to an overestimate of the cross-section for large $\tan \beta$.
} 

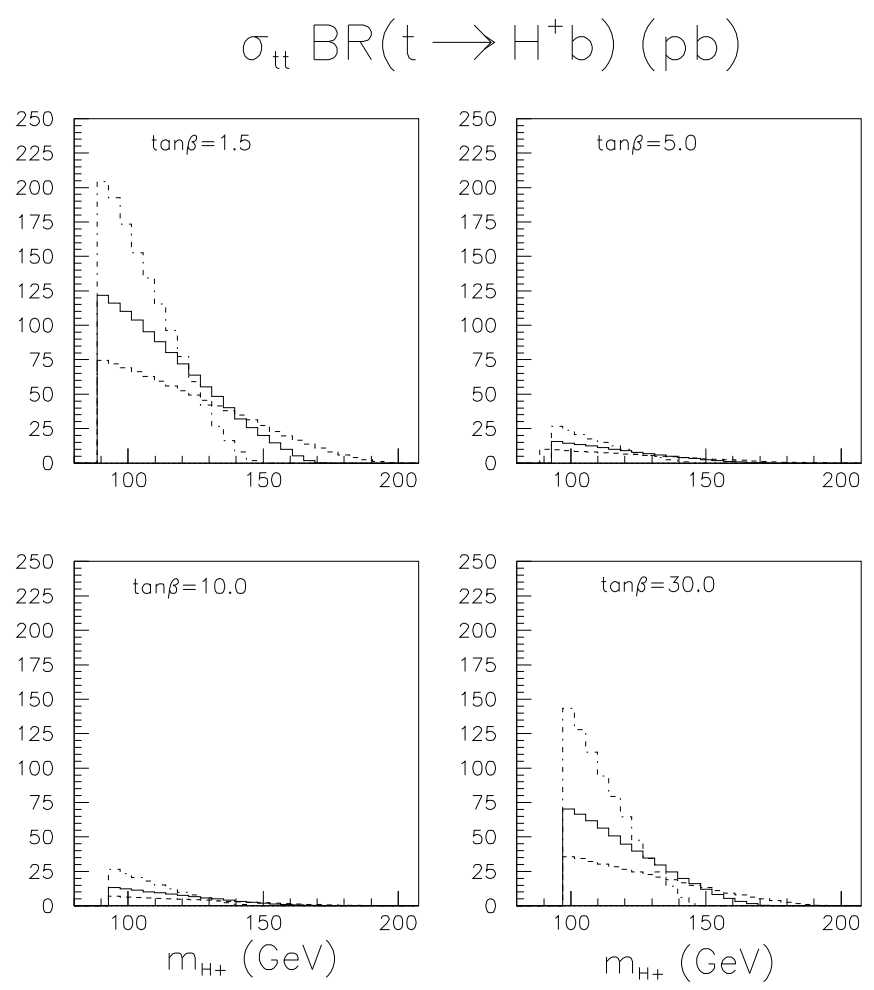

Figure 28: $\mathrm{H}^{+}$effective production cross-section through $\mathrm{t} \overline{\mathrm{t}}$ production followed by $\mathrm{t} \rightarrow \mathrm{H}^{+} \mathrm{b}$ decays as a function of $m_{\mathrm{H}^{+}}$for four values of $\tan \beta$. The solid line is for $m_{\mathrm{t}}=175 \mathrm{GeV}$, the dashed one for $m_{\mathrm{t}}=200 \mathrm{GeV}$ and the dot-dashed one for $m_{\mathrm{t}}=150 \mathrm{GeV}$.

Table 28: Expected rates $(\sigma \times B R)$ for $\mathrm{H}^{ \pm} \rightarrow \tau \nu$ decays as a function of $m_{\mathrm{A}}$, for $m_{\mathrm{t}}=175 \mathrm{GeV}$ and four different values of $\tan \beta$.

\begin{tabular}{|c||c|c|c|c|}
\hline \multicolumn{1}{|c||}{$m_{\mathrm{H}^{ \pm}}(\mathrm{GeV})$} & \multicolumn{4}{c|}{$\sigma \times B R(\mathrm{fb})$} \\
\hline & \multicolumn{4}{|c|}{ MSSM H $\mathrm{H}^{ \pm}$-boson } \\
\hline & $\tan \beta=1.5$ & $\tan \beta=5.0$ & $\tan \beta=10.0$ & $\tan \beta=30.0$ \\
\hline \hline 100.0 & 190.8 & 26.4 & 24.8 & 131.8 \\
110.0 & 168.0 & 23.3 & 19.0 & 116.8 \\
120.0 & 142.4 & 17.0 & 15.6 & 90.0 \\
130.0 & 88.0 & 13.1 & 10.4 & 64.6 \\
140.0 & 61.0 & 7.6 & 6.2 & 46.0 \\
150.0 & 36.4 & 4.8 & 3.6 & 25.0 \\
160.0 & 16.4 & 2.0 & 1.6 & 11.4 \\
\hline
\end{tabular}




\section{Description of ATLAS simulation package}

A dedicated package, developed for a systematic study of the MSSM Higgs sector, has been used throughout the study reported here. A detailed description of this code will be provided as soon as it is released for public use. This section only gives a short description of the physics inputs and algorithms.

The package consists of three main parts:

- the PYTHIA 5.707 [10] event generator, interfaced to a routine [4], which calculates the MSSM Higgs boson masses and couplings in the two-loop equivalent approximation;

- a set of semi-analytical routines, which calculate the MSSM correction factors to the production cross-sections and the MSSM branching ratios. This part uses the code from [6];

- a set of semi-analytical routines, used to interpolate the expected experimental significances in the $\left(m_{\mathrm{A}}, \tan \beta\right)$ and $\left(m_{\mathrm{h}}, \tan \beta\right)$ planes, and a semi-automatic derivation of the expected $5 \sigma$-discovery contour curves.

Whereas this package provides the means to change $m_{\mathrm{t}}$ in a flexible way, at the moment there is almost no flexibility as far as the MSSM parameters (other than $m_{\mathrm{A}}$ and $\tan \beta$ ) are concerned. In practice, the simulation assumes that mixing is minimal in the SUSY sector $\left(A_{t}=A_{b}=\mu=0\right)$ and that all SUSY particles (stops, sbottoms, charginos, neutralinos, etc.) have a mass of $\sim 1 \mathrm{TeV}$.

This package does not presently treat in a consistent way QCD corrections when evolving quark masses, the strong coupling constant $\alpha_{s}$ and decay widths, since the treatment of these issues is different in PYTHIA, [4] and [6]. The uncertainties arising from these inconsistencies are however believed to be smaller than the dominant experimental and theoretical uncertainties (higher-order corrections, $m_{\mathrm{t}}$, structure functions).

\subsection{Interface to PYTHIA}

The event generator package PYTHIA (version 5.707) has been interfaced to the code from $[4]^{6}$ through a subroutine (SUBH), which calculates the masses and the couplings in the MSSM Higgs sector in the two-loop approximation. This code is called from the PYTHIA subroutine PYINRE with the option $\operatorname{MSTP}(4)=3$. This option requires that the chosen values of $\tan \beta(\operatorname{PARU}(141))$ and of $m_{\mathrm{A}}$ (PMAS $\left.(36,1)\right)$ be given as input parameters ${ }^{7}$.

The PYTHIA code which calculates the branching ratios and the crosssections has not been modified, but the couplings that enter these calculations

\footnotetext{
${ }^{6}$ This implementation is not supported by the author of PYTHIA and not included in the official PYTHIA 5.707 version.

${ }^{7}$ The standard version of PYTHIA uses as input parameters: MSTP(4) $=2$ (tree level), $\operatorname{PARU}(141)=\tan \beta$ and $\operatorname{PMAS}(25,1)=m_{\mathrm{h}}$. For a discussion of the effect of going from tree-level to two-loop calculations, see Section 2.2.
} 
are now computed at the two-loop level.

\subsection{MSSM branching ratios and cross-sections}

This part of the package, shown schematically in Fig. 29, is based on the code of [6]. It provides MSSM correction factors to the SM Higgs production cross-sections, which are usually taken from PYTHIA, and calculates the MSSM Higgs boson decay widths and branching ratios. In the future, this code will be modified to include Higgs-boson decays to SUSY particles and non-minimal mixing in the SUSY sector.

The MSSM correction factors to couplings and tree-level processes are calculated as described in Section 2.1 (see Table 2). The MSSM correction factors to processes involving loops at the lowest order, such as $g g \rightarrow H$ or $\mathrm{H} \rightarrow \gamma \gamma$, are calculated including the contributions from ordinary fermions, charged gauge bosons, charged Higgs bosons, sfermions and charginos, but the SUSY particle masses are all fixed at $1 \mathrm{TeV}$.

The following sets of Higgs boson decay modes are treated by the package:

- $h \rightarrow \mathrm{c} \overline{\mathrm{c}}, \mathrm{b} \overline{\mathrm{b}}, \mathrm{t} \overline{\mathrm{t}}, \mathrm{gg}, \tau^{+} \boldsymbol{\tau}^{-}, \gamma \gamma, \mathrm{WW}, \mathrm{ZZ}, \mathrm{AA}, \mathrm{Z} \gamma$;

- $H \rightarrow \mathrm{c} \overline{\mathrm{c}}, \mathrm{b} \overline{\mathrm{b}}, \mathrm{t} \overline{\mathrm{t}}, \mathrm{gg}, \tau^{+} \tau^{-}, \gamma \gamma, \mathrm{WW}, \mathrm{ZZ}, \mathrm{hh}, \mathrm{AA}, \mathrm{Z} \gamma$;

- $A \rightarrow \mathrm{c} \overline{\mathrm{c}}, \mathrm{b} \overline{\mathrm{b}}, \mathrm{t} \overline{\mathrm{t}}, \mathrm{gg}, \tau^{+} \tau^{-}, \gamma \gamma, \mathrm{Zh}, \mathrm{Z} \gamma$.

This part of the package also provides the possibility to print the results of the calculations in the form of tables (LATEX format) or histograms (HBOOK format); the default version of the package provides these tables and histograms for a few values of $\tan \beta$ over the required Higgs boson mass ranges, for the $\mathrm{h}, \mathrm{H}$ and $\mathrm{A}$ decay widths, branching ratios, production crosssections and expected rates. Also provided are the SM predictions, as obtained by the PYTHIA event generator.

\subsection{Contour curves in the MSSM parameter space}

To obtain the expected sensitivity to a given signal, usually expressed as discovery contour curves, the package proceeds in the following way for each specific Higgs boson decay channel and for a given value of the experimental integrated luminosity:

1. a point is chosen in the $\left(m_{\mathrm{A}}, \tan \beta\right)$ plane;

2. the corresponding values of $m_{\mathrm{h}}$ and $m_{\mathrm{H}}$ are calculated;

3. a data file, containing the expected signal and background rates for the appropriate Higgs boson decay channel, for both low- and highluminosity operation of the ATLAS detector, is used as input to calculate by simple linear interpolation the expected sensitivity for the chosen point in parameter space; 


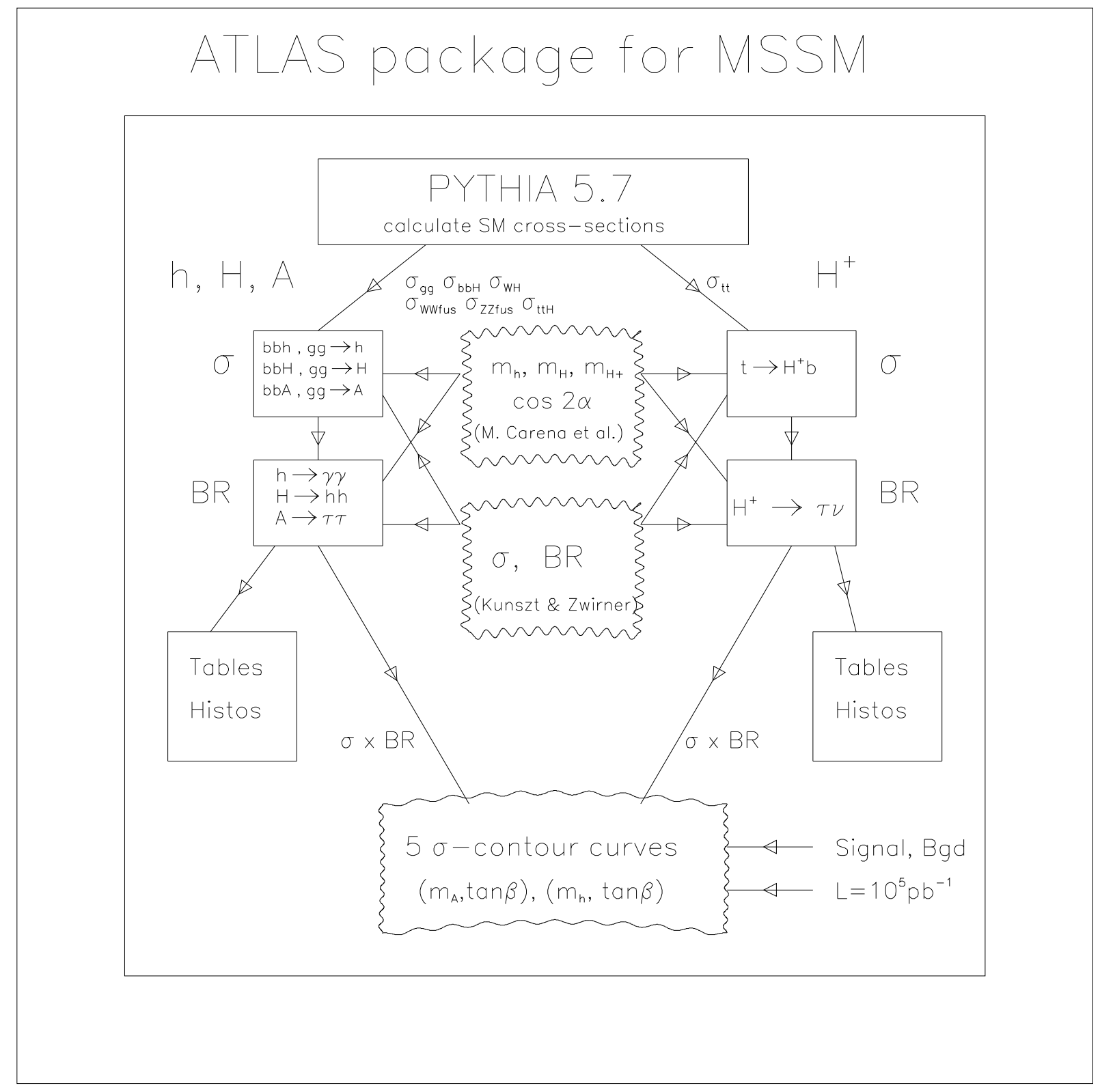

Figure 29: Schematic view of the procedure used for calculating branching ratios and cross-sections in the MSSM Higgs sector. 
4. if the expected significance is above threshold (the default value is $5 \sigma$ ), the chosen point in parameter space is validated as being observable in this channel.

This procedure, shown schematically in Fig. 30, has to be repeated for different values of $m_{\mathrm{t}}$, of the experimental integrated luminosity and of the chosen significance threshold.

\subsection{Default semi-automatic procedure}

The main steps of the complete default procedure are described below; the first three steps can be considered as initialisation steps, which do not have to be repeated when varying the experimental input:

1. a grid is defined in the $\left(m_{\mathrm{A}}, \tan \beta\right)$ plane; this grid linearly spans $m_{\mathrm{A}}$ from 50 to $500 \mathrm{GeV}$ in 225 bins of $2 \mathrm{GeV}$, and logarithmically spans $\tan \beta$ from 1 to 50 in 100 bins. Three values of $m_{\mathrm{t}}$ are chosen for the calculations, $m_{\mathrm{t}}=150,175$ and $200 \mathrm{GeV}$. Three values of the experimental integrated luminosity are used to evaluate the discovery contour curves, $3 \cdot 10^{4} \mathrm{pb}^{-1}, 10^{5} \mathrm{pb}^{-1}$ and $3 \cdot 10^{5} \mathrm{pb}^{-1}$;

2. the SM Higgs production cross-sections are computed using PYTHIA, separately for all relevant subprocesses $\left(g g \rightarrow H, W W_{f u s}, Z Z_{\text {fus }}\right.$, $\mathrm{b} \overline{\mathrm{b}} H, \mathrm{t} \overline{\mathrm{t}} H, W H, Z H$ and $\mathrm{t} \overline{\mathrm{t}})$. For the $g g \rightarrow H$, $\mathrm{t} \overline{\mathrm{t}} H$ and $\mathrm{t} \overline{\mathrm{t}}$ subprocesses, the cross-sections are computed for the three chosen values of $m_{\mathrm{t}}$. The results are stored in histograms as a function of the Higgs-boson masses;

3. the corresponding MSSM branching ratios and production cross-sections are then computed as described above, for each subprocess and for all relevant Higgs boson states. The total expected rates $(\sigma \times B R)$ are then stored in 2-dimensional arrays corresponding to the chosen grid in the $\left(m_{\mathrm{A}}, \tan \beta\right)$ plane. The values of $m_{\mathrm{h}}$ and $m_{\mathrm{H}}$ corresponding to each point in the grid are also stored;

4. the input information from detailed physics simulations of the various channels is read in. This contains the expected numbers of signal (S) and background (B) events for a few values of the Higgs boson masses. Due to the variation of detector performance (resolution, efficiency and kinematic thresholds for triggering and reconstruction) as a function of luminosity, the expected values of $S$ and $B$ are given separately for low- $\left(3 \cdot 10^{4} \mathrm{pb}^{-1}\right)$ and high- $\left(10^{5} \mathrm{pb}^{-1}\right)$ luminosity operation. The input files also provide the range of Higgs boson masses over which the simulation results can be extrapolated; for example, values of $m_{\mathrm{h}}$ lower than 60 to $70 \mathrm{GeV}$ are not assumed to be observable at the present stage. Finally, they provide the expected mass resolution and relevant Higgs boson width for each mass value; 


\section{ATLAS package for $5 \sigma$-contour curves}

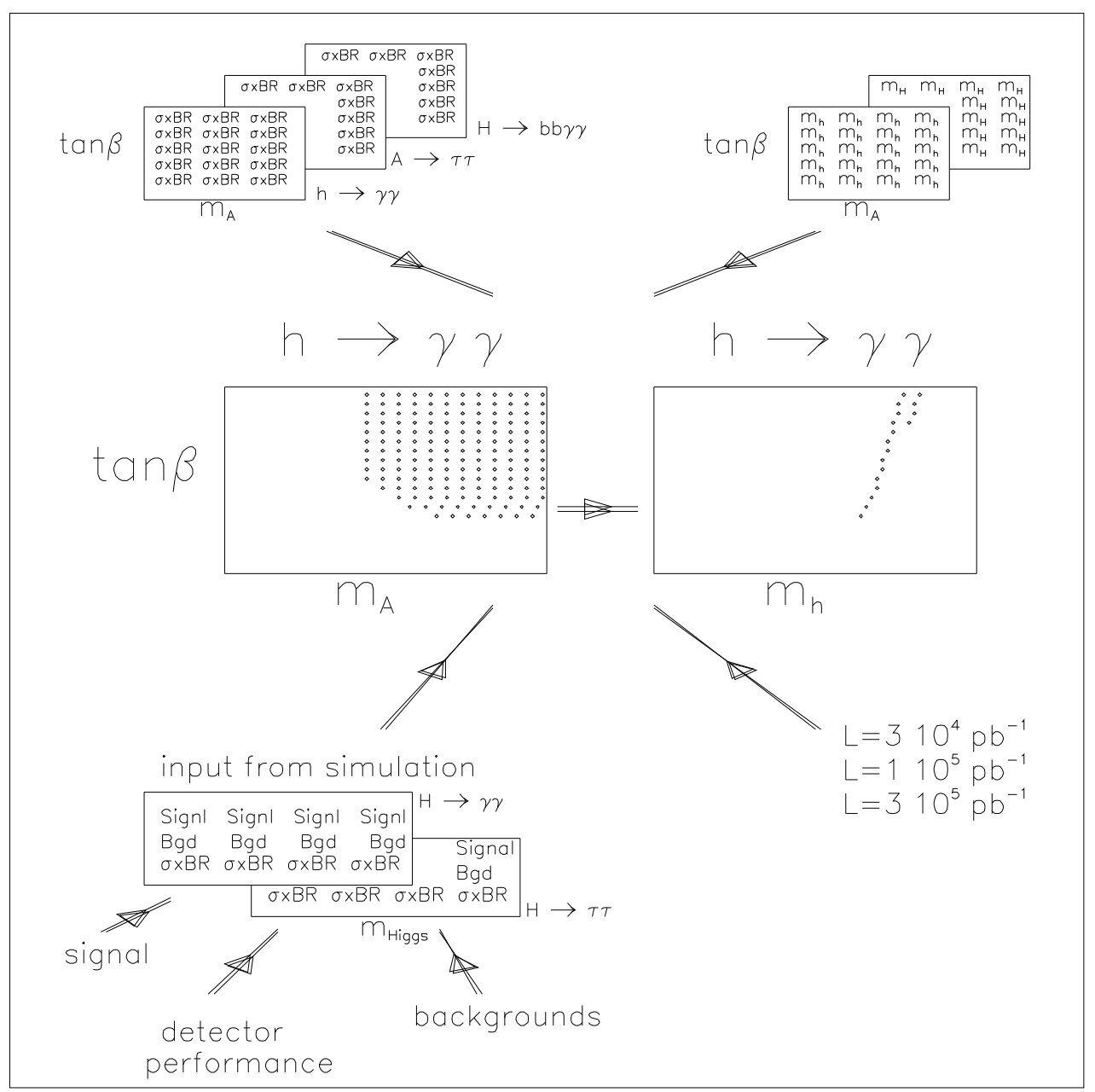

Figure 30: Schematic view of the procedure used for deriving the $5 \sigma$ discovery contour curves in the MSSM Higgs sector. 
5. the expected significance of each signal channel is calculated for each point of the grid. The usual naive estimator $S / \sqrt{B}$ is used in all cases, except if both $\mathrm{S}$ and $\mathrm{B}$ are smaller than 25, in which case Poisson statistics are used to compute the equivalent Gaussian significance. If the result exceeds $5 \sigma$, the corresponding bin in a 2-dimensional histogram representing the grid in the $\left(m_{\mathrm{A}}, \tan \beta\right)$ parameter space is set to 1 . For the more complicated $H \rightarrow h h$ and $A \rightarrow Z h$ channels, the interpolation procedure is 2-dimensional, since two MSSM Higgs bosons are involved. For the $\mathrm{A} \rightarrow \tau \tau$ and $\mathrm{H} \rightarrow \tau \tau$ channels, the signals overlap completely for $m_{\mathrm{A}}>140 \mathrm{GeV}$ but much less so for smaller values of $m_{\mathrm{A}}$. The impact of this incomplete or complete overlap of the two signal channels is properly taken into account in the calculation of the expected overall significance. When the Higgs boson width is not negligible with respect to the experimental width, the experimental input is rescaled accordingly;

6. the results in the $\left(m_{\mathrm{A}}, \tan \beta\right)$ plane are mapped onto the $\left(m_{\mathrm{h}}, \tan \beta\right)$ plane;

7. the final 2-dimensional histograms are saved for each channel separately and used to draw the $5 \sigma$-discovery contour curves with PAW, as a function of integrated luminosity and of $m_{\mathrm{t}}$;

8. ATLAS and CMS are combined independently, assuming the same sensitivity for all channels except $\mathrm{H} \rightarrow \gamma \gamma$, for which CMS is assumed to have $30 \%$ (resp. 10\%) better sensitivity at low (resp. high) luminosity [11]. 


\section{$5 \quad$ Observability of MSSM Higgs bosons}

There are many processes that could provide evidence for one or more MSSM Higgs bosons at the LHC. Several of them have been studied for the TP:

- $\mathrm{h} \rightarrow \gamma \gamma, \mathrm{H} \rightarrow \gamma \gamma$ and Wh, thth with $W \rightarrow l \nu$ and $\mathrm{h} \rightarrow \gamma \gamma$,

- $\mathrm{H} \rightarrow \mathrm{ZZ}^{(\star)} \rightarrow 4 \ell$

- $\mathrm{H} \rightarrow \tau \tau$ and $\mathrm{A} \rightarrow \tau \tau$,

- $\mathrm{t} \overline{\mathrm{t}}$ with $t \rightarrow H^{+} b$ and $H^{+} \rightarrow \tau \nu$.

Since the TP, a number of other possible channels have been studied:

- Wh and tîh with $\mathrm{h} \rightarrow \mathrm{b} \overline{\mathrm{b}}$,

- $\mathrm{H} \rightarrow \mu \mu$ and $\mathrm{A} \rightarrow \mu \mu$,

- $\mathrm{H} \rightarrow \mathrm{t} \overline{\mathrm{t}}$ and $\mathrm{A} \rightarrow \mathrm{t} \overline{\mathrm{t}}$,

- $\mathrm{H} \rightarrow \mathrm{hh}$ with $\mathrm{h} \rightarrow \mathrm{b} \overline{\mathrm{b}}$ and $\mathrm{h} \rightarrow \gamma \gamma$,

- $\mathrm{A} \rightarrow \mathrm{Zh}$ with $\mathrm{h} \rightarrow \mathrm{b} \overline{\mathrm{b}}$ and $\mathrm{Z} \rightarrow \ell \ell$.

A complete study of any given channel requires accurate estimates of the expected signal rates, including the detector acceptance as well as its reconstruction efficiency and mass resolution (in most cases), and thorough calculations of the various backgrounds, whether irreducible, i.e. corresponding to the same final state as the signal, or not.

This Section briefly describes the results of the simulations leading to the expected signal and background rates, referring to separate work or to the relevant Appendix to this report for more detailed discussions. For all signal channels, only SM background sources were considered. For several signal channels, the expected observability can be simply rescaled from studies of the corresponding SM channel $\left(\mathrm{H} \rightarrow \gamma \gamma, \mathrm{H} \rightarrow \mathrm{b} \bar{b}\right.$ and $\left.\mathrm{H} \rightarrow \mathrm{ZZ}^{(\star)} \rightarrow 4 \ell\right)$.

The procedure described in Section 4 was used consistently throughout the study, and the experimental inputs to this procedure, i.e. the expected signal and background rates at low and high luminosity, are presented separately below for each channel, for a few relevant values of the corresponding Higgs boson mass. The results are presented as $5 \sigma$-discovery contour curves in the $\left(m_{\mathrm{A}}, \tan \beta\right)$ and $\left(m_{\mathrm{h}}, \tan \beta\right)$ planes, both as a function of integrated luminosity $\left(3 \cdot 10^{4} \mathrm{pb}^{-1}, 10^{5} \mathrm{pb}^{-1} \text { and } 3 \cdot 10^{5} \mathrm{pb}^{-1}\right)^{8}$ and of the top-quark mass $m_{\mathrm{t}}\left(m_{\mathrm{t}}=150,175\right.$ and $\left.200 \mathrm{GeV}\right)$. In the case of the $\left(m_{\mathrm{h}}, \tan \beta\right)$ plane, some values are excluded theoretically and the corresponding area is crosshatched in the plots.

\footnotetext{
${ }^{8}$ For consistency, it is assumed that $10^{5} \mathrm{pb}^{-1}$ consists of $3 \cdot 10^{4} \mathrm{pb}^{-1}$ (low luminosity operation) $+0.7 \cdot 10^{5} \mathrm{pb}^{-1}$ (high luminosity operation), and similarly that $3 \cdot 10^{5} \mathrm{pb}^{-1}$ consists of $3 \cdot 10^{4} \mathrm{pb}^{-1}+2.7 \cdot 10^{5} \mathrm{pb}^{-1}$.
} 


\subsection{The $\mathrm{h} \rightarrow \gamma \gamma$ and $\mathrm{H} \rightarrow \gamma \gamma$ channels}

The most recent results on the observability of the inclusive $H \rightarrow \gamma \gamma$ channel with the ATLAS detector have been described in detail in [3] for the case of a SM Higgs boson. The reader will find in [3] the definition of the selection criteria and a justification of the expected performance of the ATLAS detector in this channel. In particular, one should note that the photon identification efficiency, the reducible background rejection and the diphoton mass resolution were obtained from a full simulation of the ATLAS detector.

For Higgs boson masses between 80 and $150 \mathrm{GeV}$, Table 29 shows the expected signal and background rates and the expected significances, for integrated luminosities of $3 \cdot 10^{4} \mathrm{pb}^{-1}$ (low luminosity operation) and $10^{5} \mathrm{pb}^{-1}$ (high luminosity operation). The low luminosity performance of the ATLAS detector is improved with respect to the high luminosity performance in terms of photon identification efficiency ( $85 \%$ compared to $80 \%$ ) and of diphoton mass resolution $\left(1.05 \mathrm{GeV}\right.$ compared to $1.25 \mathrm{GeV}$ for $\left.m_{\mathrm{H}}=100 \mathrm{GeV}\right)$.

The search for the SM Higgs boson in $\mathrm{H} \rightarrow \gamma \gamma$ decays can also be performed using $\mathrm{WH}$ and $\mathrm{t} \overline{\mathrm{t}} \mathrm{H}$ production, for events with a high- $\mathrm{p}_{\mathrm{T}}$ isolated lepton from $\mathrm{W}$-decay in addition to the two photons from Higgs decay. The signal-tobackground ratio is much higher in this channel than in the inclusive $\mathrm{H} \rightarrow \gamma \gamma$ channel, as shown in Table 30, obtained by extrapolating the results of [12]. The signal rates, however, are too low in this channel for it to be observed with integrated luminosities much smaller than $3 \cdot 10^{5} \mathrm{pb}^{-1}$. Nevertheless, the expected sensitivity to this channel can be combined with that for the inclusive channel to improve the overall sensitivity to a possible signal.

The expected MSSM rates, for both $\mathrm{h} \rightarrow \gamma \gamma$ and $\mathrm{H} \rightarrow \gamma \gamma$ decays, are generally suppressed with respect to the SM case, and are only comparable to the SM rates over a very limited mass range for the Higgs boson under consideration (see Sections 3.1 and 3.2). As explained in Section 4, the rates and significances quoted in Tables 29 and 30 are used as input values to the procedure that determines the observability of this channel in the MSSM case. To obtain $5 \sigma$-discovery contour curves in the $\left(m_{\mathrm{A}}, \tan \beta\right)$ and $\left(m_{\mathrm{h}}, \tan \beta\right)$ planes, only h-boson masses larger than $70 \mathrm{GeV}$ were considered, since a proper experimental study of signal acceptance and background rates has not been performed for masses much below $80 \mathrm{GeV}$.

The expected $5 \sigma$-discovery contour curves for ATLAS are shown in the following pages, as a function of integrated luminosity and of $m_{\mathrm{t}}$ :

- Figs. 31 to 34 for the $\mathrm{h} \rightarrow \gamma \gamma$ inclusive channel. Fig. 31 shows that the observability in the $\left(m_{\mathrm{A}}, \tan \beta\right)$ plane depends critically on the integrated luminosity, which just reflects the very slow variation of $m_{\mathrm{h}}$ and of $\sigma \times B R$ as $m_{\mathrm{A}}$ increases. Fig. 33 illustrates this fact in the $\left(m_{\mathrm{h}}, \tan \beta\right)$ plane and shows that, as expected from Table 29, a $5 \sigma$ discovery cannot be achieved for $m_{\mathrm{h}}<90 \mathrm{GeV}$, even with the largest integrated luminosity considered here. As shown in Figs. 32 and 34, the observability improves significantly as $m_{\mathrm{t}}$ increases, due to the larger 
values of $m_{\mathrm{h}}$ expected for given values of $m_{\mathrm{A}}$ and $\tan \beta$.

- Figs. 35 to 37 for the $\mathrm{h} \rightarrow \gamma \gamma$ associated channel, which, as discussed above, is only accessible for an integrated luminosity of $3 \cdot 10^{5} \mathrm{pb}^{-1}$. In contrast to the $\mathrm{h} \rightarrow \gamma \gamma$ inclusive channel, the sensitivity extends down to $\tan \beta=1$, for $m_{\mathrm{t}}=175 \mathrm{GeV}$ and for values of $m_{\mathrm{A}}$ larger than 150 to $200 \mathrm{GeV}$, as shown in Fig. 35. Fig. 36 shows that the observability of this channel is not very sensitive to the value of $m_{\mathrm{t}}$, except for $m_{\mathrm{t}}=150 \mathrm{GeV}$ and $\tan \beta<2$. This is due to the fact that $m_{\mathrm{h}}$ is smaller than $70 \mathrm{GeV}$ in this case, as can be seen clearly in Fig. 37.

- Figs. 38 to 41 for the combined $\mathrm{h} \rightarrow \gamma \gamma$ inclusive and associated channels. Fig. 38 shows that a combined $5 \sigma$-discovery is possible for all values of $\tan \beta$ provided $m_{\mathrm{A}}$ is larger than $180 \mathrm{GeV}$ (resp. $300 \mathrm{GeV}$ ), for an integrated luminosity of $3 \cdot 10^{5} \mathrm{pb}^{-1}$ (resp. $10^{5} \mathrm{pb}^{-1}$ ). Fig. 39 shows that the combined sensitivity does not vary much with $m_{\mathrm{t}}$, except for $\tan \beta<2$ and $m_{\mathrm{t}}=150 \mathrm{GeV}$, as for the associated channel.

- Figs. 42 to 45 for the inclusive $\mathrm{H} \rightarrow \gamma \gamma$ channel. These figures are included mainly for completeness, since the $m_{\mathrm{H}}$ range accessible in this channel is quite small, although it gets wider as $m_{\mathrm{t}}$ increases.

Figs. 38 to 41 deserve several further remarks:

- in the $\left(m_{\mathrm{A}}, \tan \beta\right)$ plane, the position of the $5 \sigma$-discovery contour curves is uncertain to $\sim \pm 30 \mathrm{GeV}$ along the $m_{\mathrm{A}}$-axis, due to the theoretical uncertainties still inherent in the calculation of $m_{\mathrm{h}}$ as a function of $m_{\mathrm{A}}$;

- the $\mathrm{h} \rightarrow \gamma \gamma$ branching ratio was computed assuming that all SUSY particles have a mass of $1000 \mathrm{GeV}$. More realistic mass spectra of SUSY particles usually contain lighter stop-quarks and charginos, and this may significantly decrease the $h \rightarrow \gamma \gamma$ branching ratio [13]. Possible decays of the h-boson to the lightest neutralino may also affect the $\mathrm{h} \rightarrow \gamma \gamma$ branching ratio $[9]$;

- SUSY particle masses lighter than $1000 \mathrm{GeV}$ could strongly affect the $\mathrm{gg} \rightarrow \mathrm{h}$ production cross-section. In fact, for some specific choices of the SUSY model parameters, this cross-section could decrease by more than an order of magnitude. The $\mathrm{h} \rightarrow \gamma \gamma$ channel could then only be observed at the LHC through Wh and tth production with a somewhat reduced sensitivity but not overly so. This emphasises the need for a more systematic study of this channel in the near future;

- since the $\mathrm{SM}$ and MSSM h/H $\rightarrow \gamma \gamma$ rates are very similar over the accessible region in the $\left(m_{\mathrm{A}}, \tan \beta\right)$ plane, the observation of $\mathrm{h} / \mathrm{H} \rightarrow \gamma \gamma$ decays alone at the LHC will not be sufficient to demonstrate the existence of a non-SM Higgs sector. 
Table 29: Observability of the SMH $\rightarrow \gamma \gamma$ for the inclusive channel at low and high luminosities. The expected numbers of signal and background events and significances are taken from Table 21 of [3].

\begin{tabular}{|c|c||c|c|c||c|c|c|}
\hline \multicolumn{2}{|c||}{} & \multicolumn{3}{c||}{ Low luminosity } & \multicolumn{3}{c|}{ High luminosity } \\
\cline { 3 - 8 } & \multicolumn{3}{c|}{$3 \cdot 10^{4} \mathrm{pb}^{-1}$} & \multicolumn{3}{c|}{$\mathrm{pb}^{-1}$} \\
\hline $\begin{array}{c}m_{\mathrm{H}} \\
(\mathrm{GeV})\end{array}$ & $\begin{array}{c}\sigma \times B R \\
(\mathrm{fb})\end{array}$ & Signal & Background & Signif. & Signal & Background & Signif. \\
\hline \hline 80.0 & 32.3 & 185 & 14300 & 1.5 & 545 & 51200 & 2.4 \\
90.0 & 36.6 & 209 & 12500 & 1.9 & 618 & 44700 & 2.9 \\
100.0 & 40.7 & 322 & 13700 & 2.7 & 951 & 48800 & 4.3 \\
110.0 & 44.7 & 365 & 10400 & 3.6 & 1077 & 37000 & 5.6 \\
120.0 & 46.3 & 392 & 8900 & 4.1 & 1156 & 31800 & 6.5 \\
130.0 & 42.9 & 370 & 7600 & 4.2 & 1092 & 27100 & 6.6 \\
150.0 & 23.5 & 219 & 5300 & 3.0 & 646 & 18800 & 4.7 \\
\hline
\end{tabular}

Table 30: Observability of the $S M \mathrm{H} \rightarrow \gamma \gamma$, for the associated channel (WH and $\mathrm{t} \overline{\mathrm{t}} \mathrm{H}$ with $\mathrm{W} \rightarrow \ell \nu)$, at low and high luminosities. The expected numbers of signal and background events are taken from Table 11.8 of the ATLAS TP. The significances are estimated as described in Section 4.

\begin{tabular}{|c|c||c|c|c||c|c|c|}
\hline \multicolumn{2}{|c||}{} & \multicolumn{3}{c||}{ Low luminosity } & \multicolumn{3}{c|}{ High luminosity } \\
\cline { 2 - 7 } \multicolumn{2}{|c||}{} & \multicolumn{3}{c|}{$3 \cdot 10^{4} \mathrm{pb}^{-1}$} & \multicolumn{3}{c|}{$\mathrm{pb}^{-1}$} \\
\hline $\begin{array}{c}m_{\mathrm{H}} \\
(\mathrm{GeV})\end{array}$ & $\begin{array}{c}\sigma \times B R \\
(\mathrm{fb})\end{array}$ & Signal & Background & Signif. & Signal & Background & Signif. \\
\hline \hline 80.0 & 1.17 & 5 & 4 & 2.0 & 15 & 13.4 & 3.4 \\
100.0 & 1.10 & 5 & 4 & 2.0 & 15 & 13.4 & 3.4 \\
120.0 & 0.84 & 5 & 4 & 2.0 & 15 & 13.4 & 3.4 \\
\hline
\end{tabular}




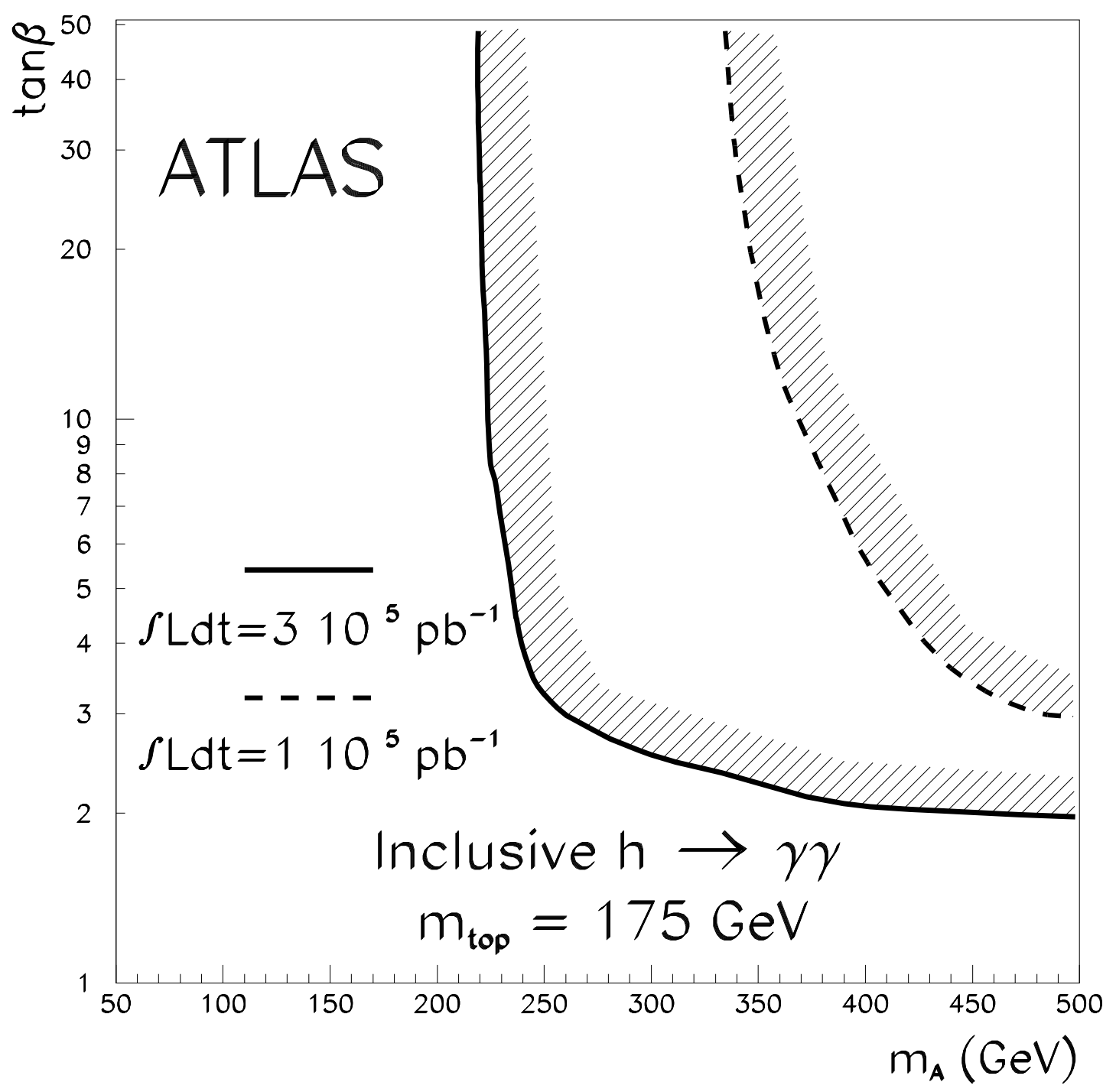

Figure 31: For $m_{\mathrm{t}}=175 \mathrm{GeV}$ and integrated luminosities of $10^{5} \mathrm{pb}^{-1}$ and $3 \cdot 10^{5} \mathrm{pb}^{-1}, 5 \sigma$-discovery contour curves for the $\mathrm{h} \rightarrow \gamma \gamma$ inclusive channel in the $\left(m_{\mathrm{A}}, \tan \beta\right)$ plane. 


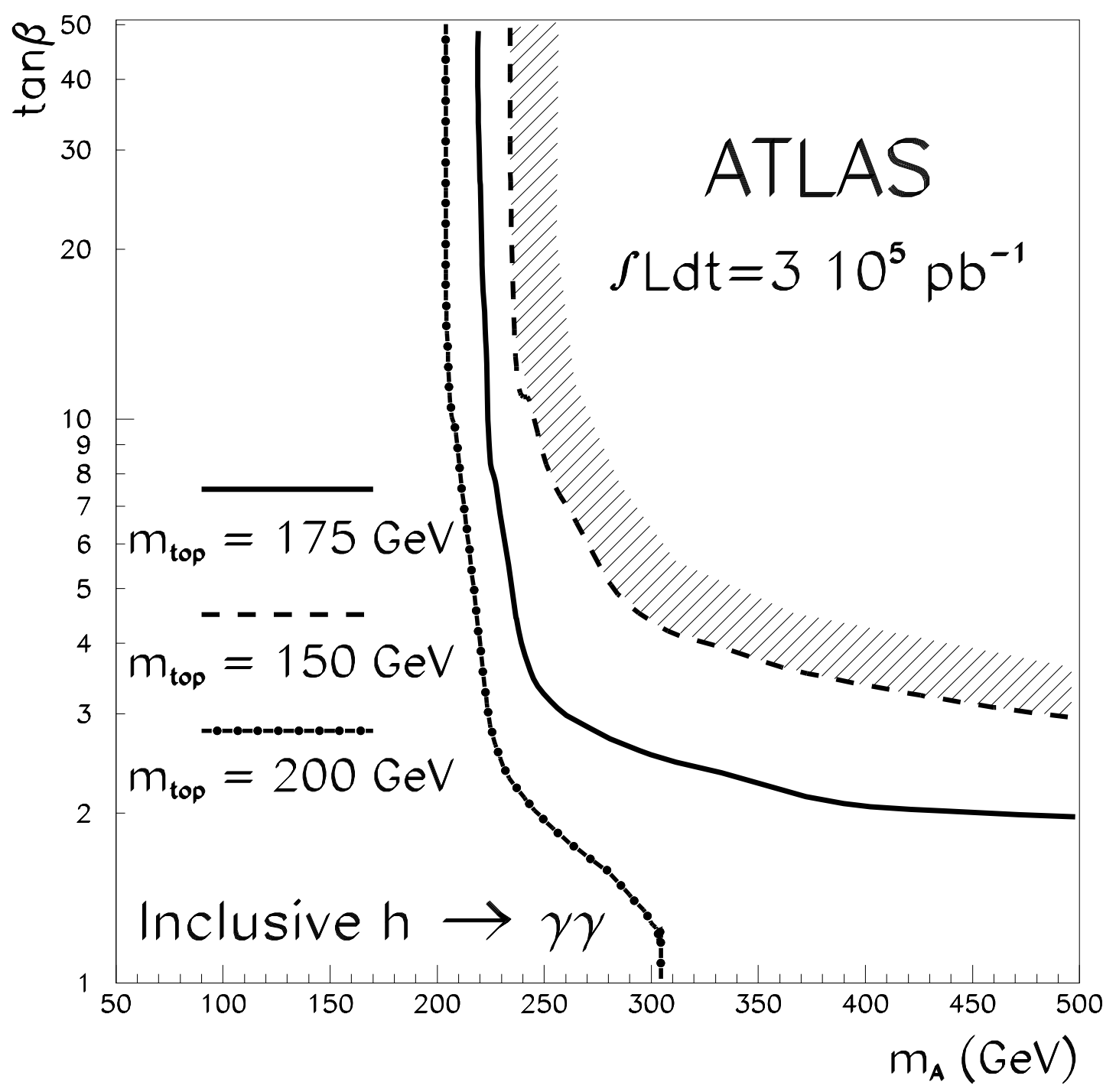

Figure 32: For $m_{\mathrm{t}}=150,175$ and $200 \mathrm{GeV}$ and an integrated luminosity of $3 \cdot 10^{5} \mathrm{pb}^{-1}, 5 \sigma$-discovery contour curves for the $\mathrm{h} \rightarrow \gamma \gamma$ inclusive channel in the $\left(m_{\mathrm{A}}, \tan \beta\right)$ plane. 




Figure 33: For $m_{\mathrm{t}}=175 \mathrm{GeV}$ and integrated luminosities of $10^{5} \mathrm{pb}^{-1}$ and $3 \cdot 10^{5} \mathrm{pb}^{-1}, 5 \sigma$-discovery contour curves for the $\mathrm{h} \rightarrow \gamma \gamma$ inclusive channel in the $\left(m_{\mathrm{h}}, \tan \beta\right)$ plane. The cross-hatched area is theoretically excluded. 


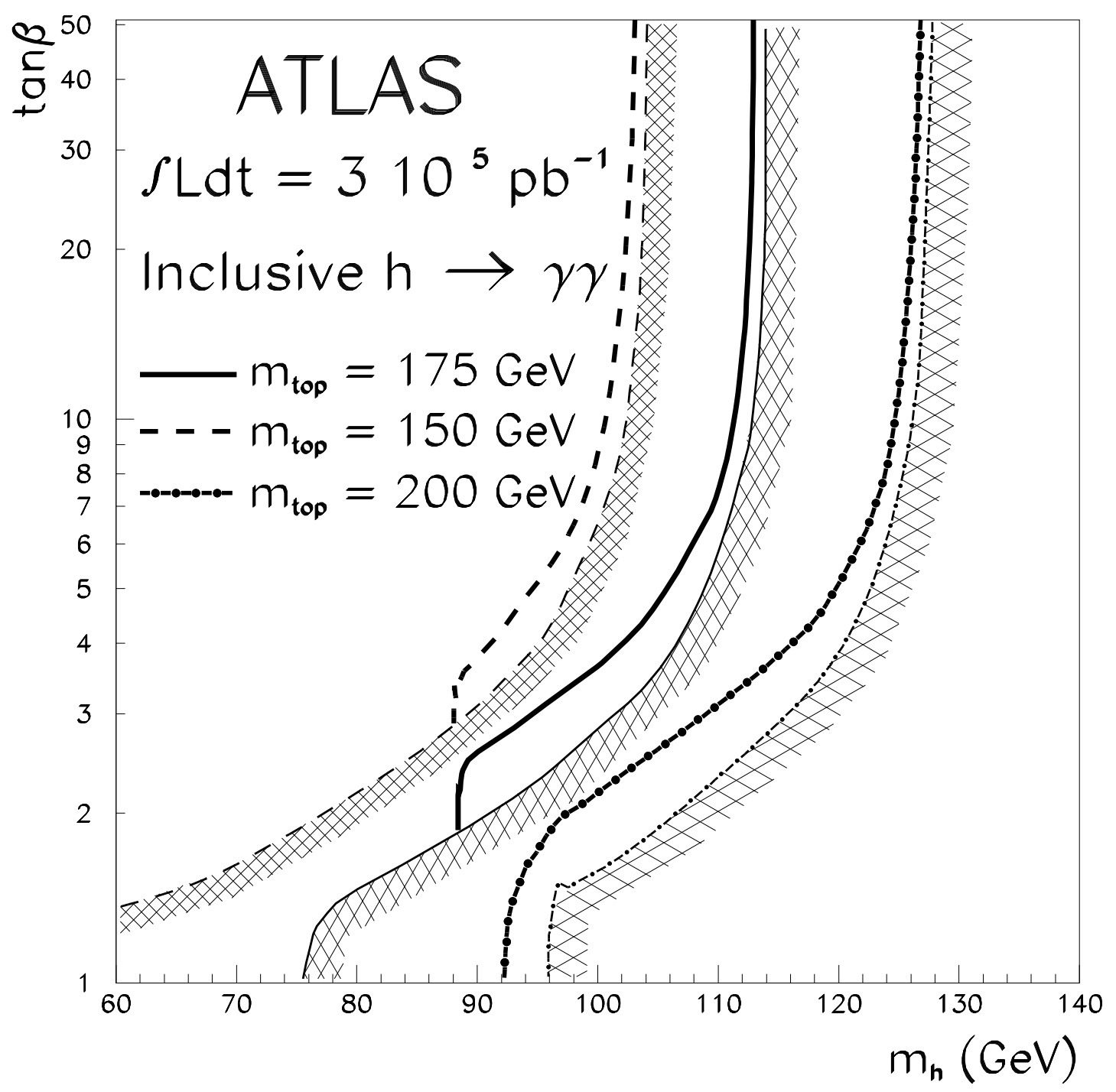

Figure 34: For $m_{\mathrm{t}}=150,175$ and $200 \mathrm{GeV}$ and an integrated luminosity of $3 \cdot 10^{5} \mathrm{pb}^{-1}, 5 \sigma$-discovery contour curves for the $\mathrm{h} \rightarrow \gamma \gamma$ inclusive channel in the $\left(m_{\mathrm{h}}, \tan \beta\right)$ plane. The cross-hatched areas are theoretically excluded. 


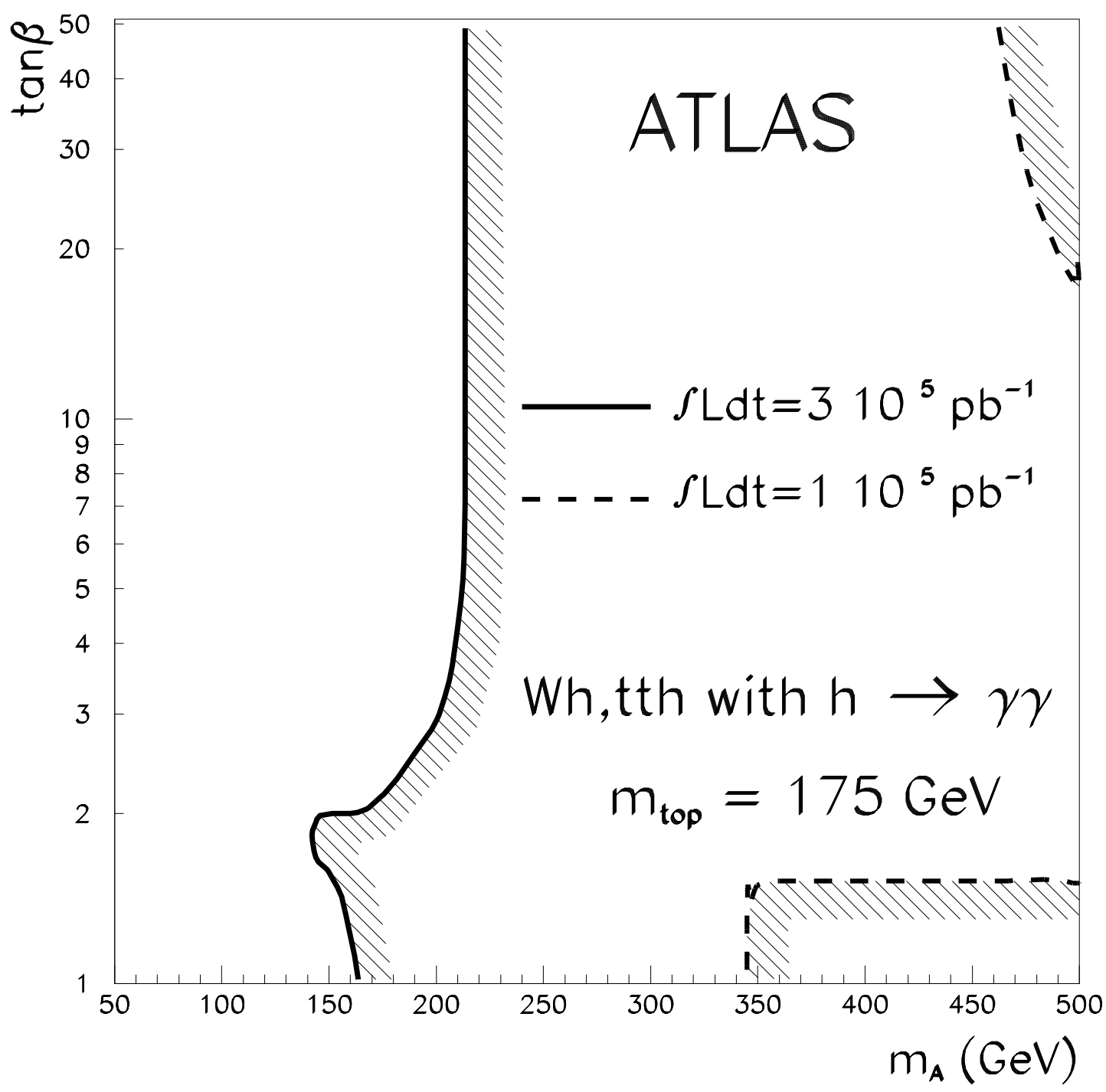

Figure 35: For $m_{\mathrm{t}}=175 \mathrm{GeV}$ and integrated luminosities of $10^{5} \mathrm{pb}^{-1}$ and $3 \cdot 10^{5} \mathrm{pb}^{-1}, 5 \sigma$-discovery contour curves for the $\mathrm{h} \rightarrow \gamma \gamma$ associated channel in the $\left(m_{\mathrm{A}}, \tan \beta\right)$ plane. 


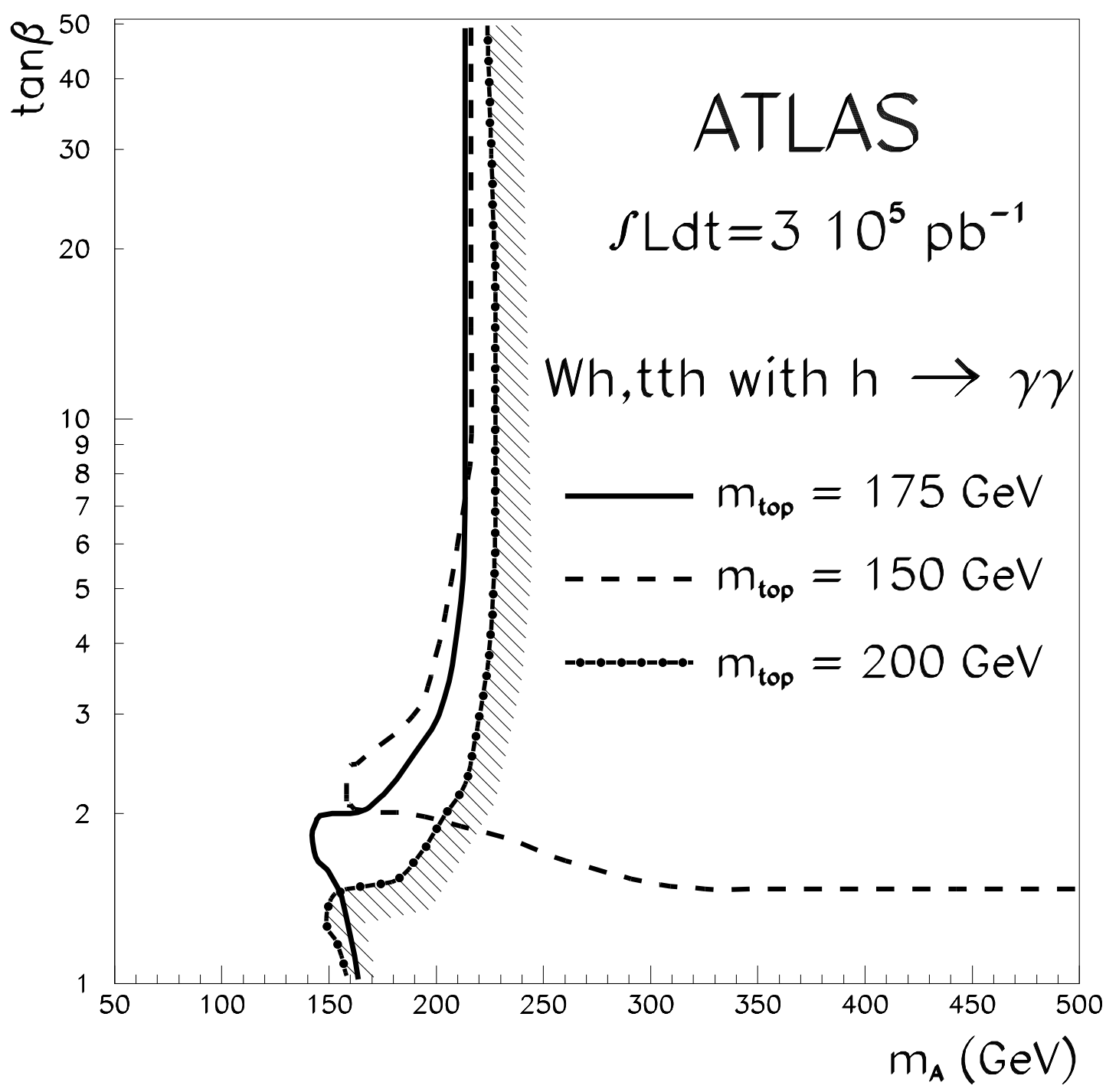

Figure 36: For $m_{\mathrm{t}}=150,175$ and $200 \mathrm{GeV}$ and an integrated luminosity of $3 \cdot 10^{5} \mathrm{pb}^{-1}, 5 \sigma$-discovery contour curves for the $\mathrm{h} \rightarrow \gamma \gamma$ associated channel in the $\left(m_{\mathrm{A}}, \tan \beta\right)$ plane. 


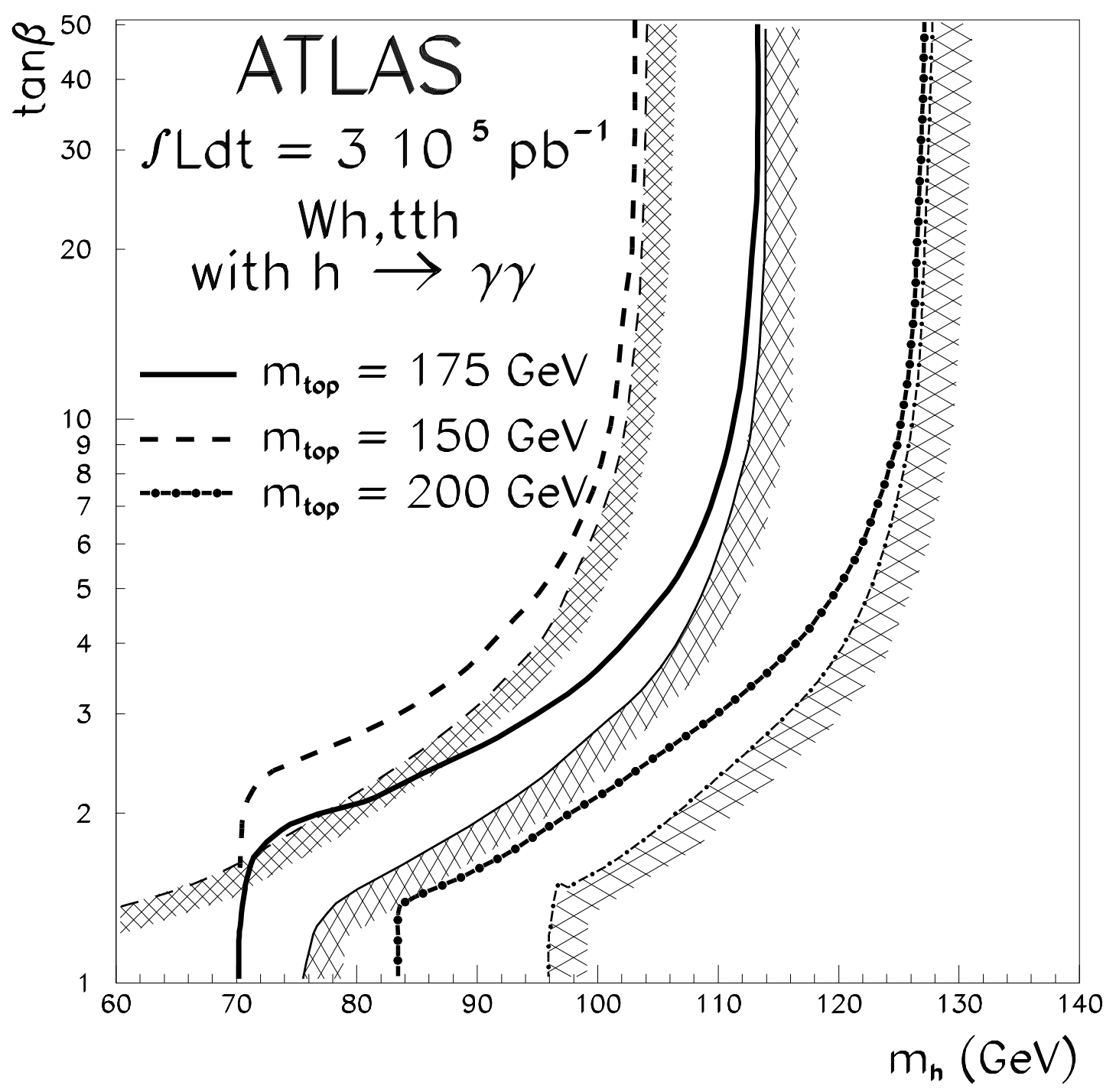

Figure 37: For $m_{\mathrm{t}}=150,175$ and $200 \mathrm{GeV}$ and an integrated luminosity of $3 \cdot 10^{5} \mathrm{pb}^{-1}, 5 \sigma$-discovery contour curves for the $\mathrm{h} \rightarrow \gamma \gamma$ associated channel in the $\left(m_{\mathrm{h}}, \tan \beta\right)$ plane. The cross-hatched areas are theoretically excluded. 


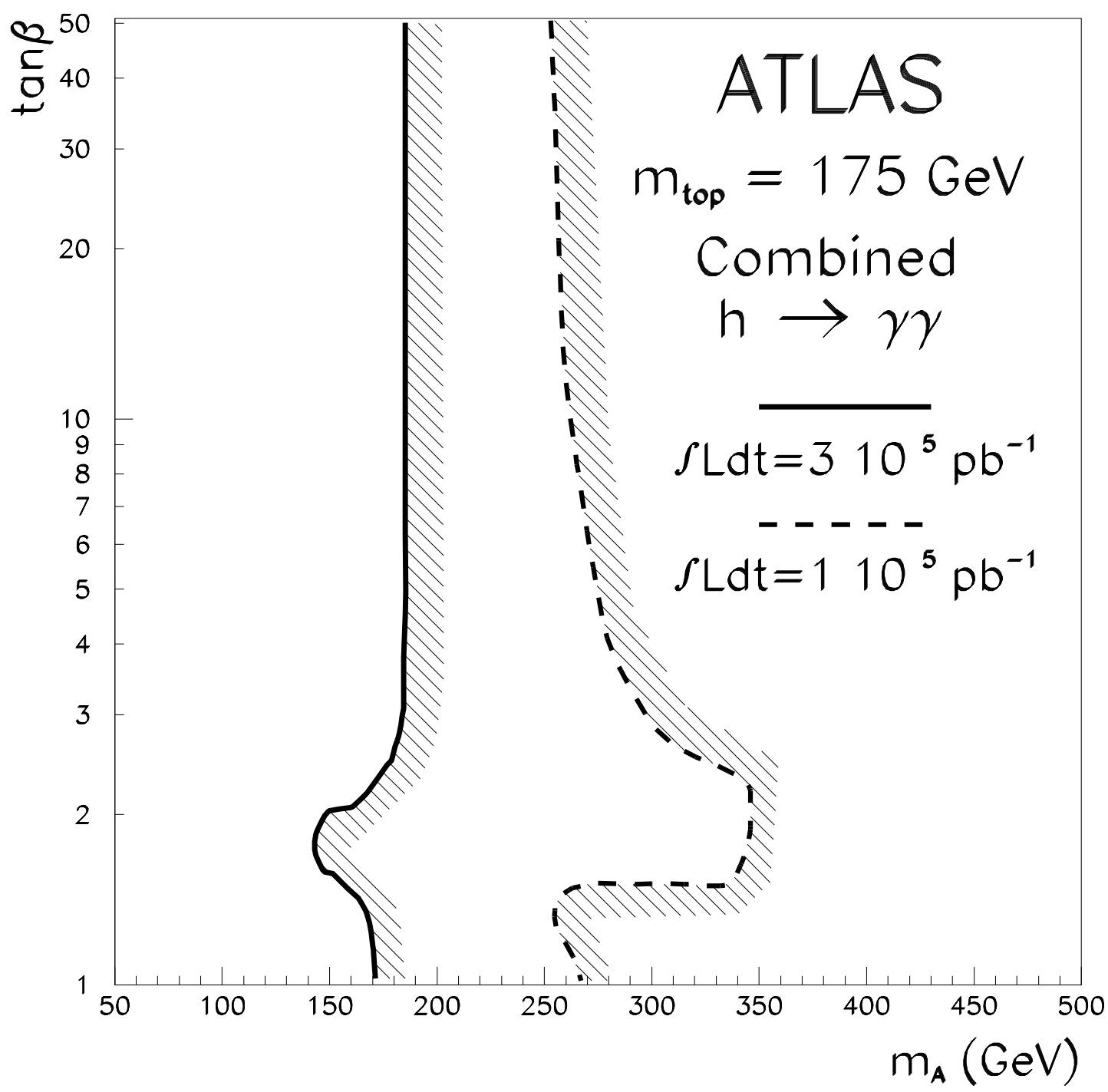

Figure 38: For $m_{\mathrm{t}}=175 \mathrm{GeV}$ and integrated luminosities of $10^{5} \mathrm{pb}^{-1}$ and $3 \cdot 10^{5} \mathrm{pb}^{-1}, 5 \sigma$-discovery contour curves for the combined $\mathrm{h} \rightarrow \gamma \gamma$ inclusive and associated channels in the $\left(m_{\mathrm{A}}, \tan \beta\right)$ plane. 


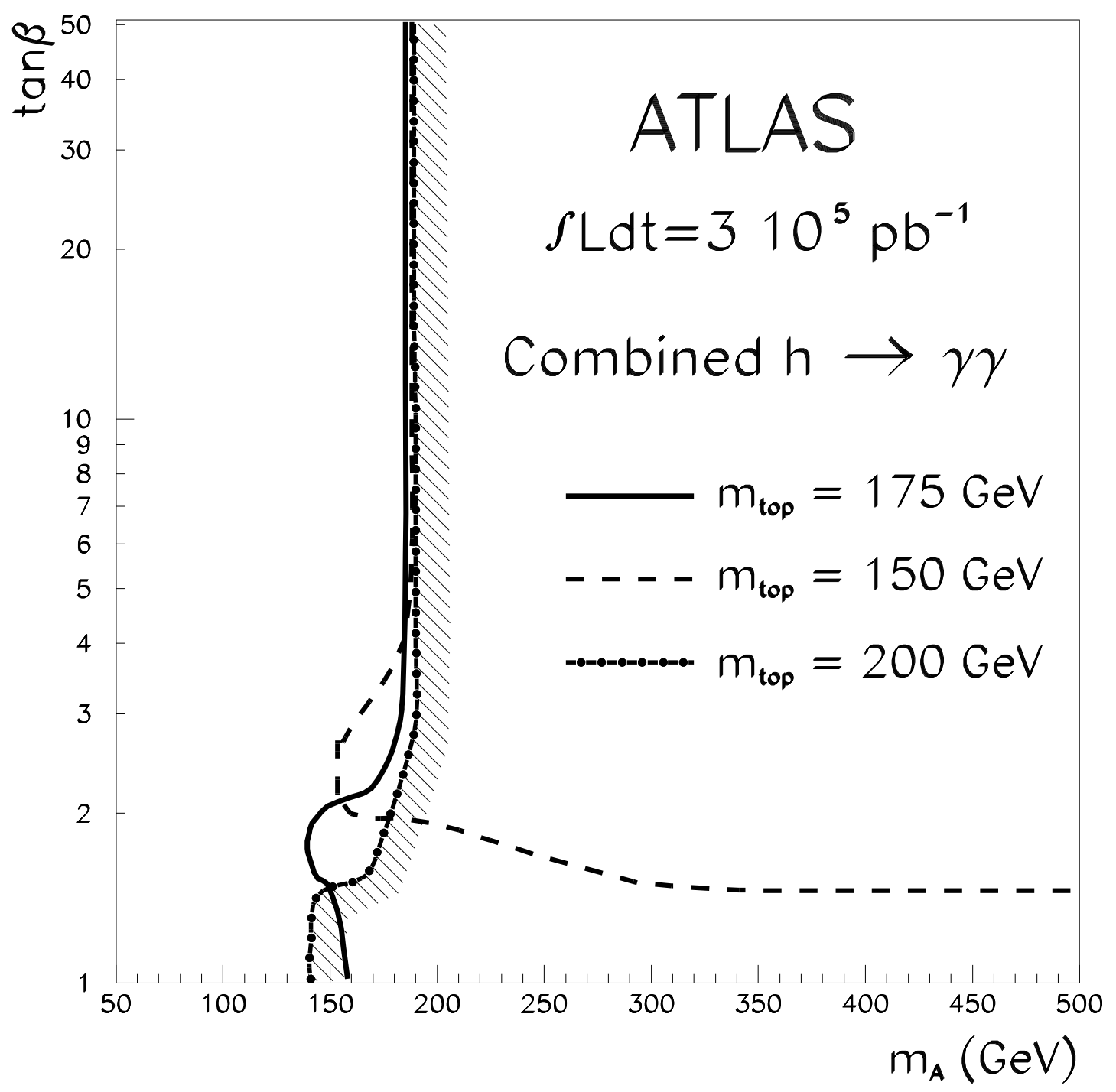

Figure 39: For $m_{\mathrm{t}}=150,175$ and $200 \mathrm{GeV}$ and an integrated luminosity of $3 \cdot 10^{5} \mathrm{pb}^{-1}, 5 \sigma$-discovery contour curves for the combined $\mathrm{h} \rightarrow \gamma \gamma$ inclusive and associated channels in the $\left(m_{\mathrm{A}}, \tan \beta\right)$ plane. 


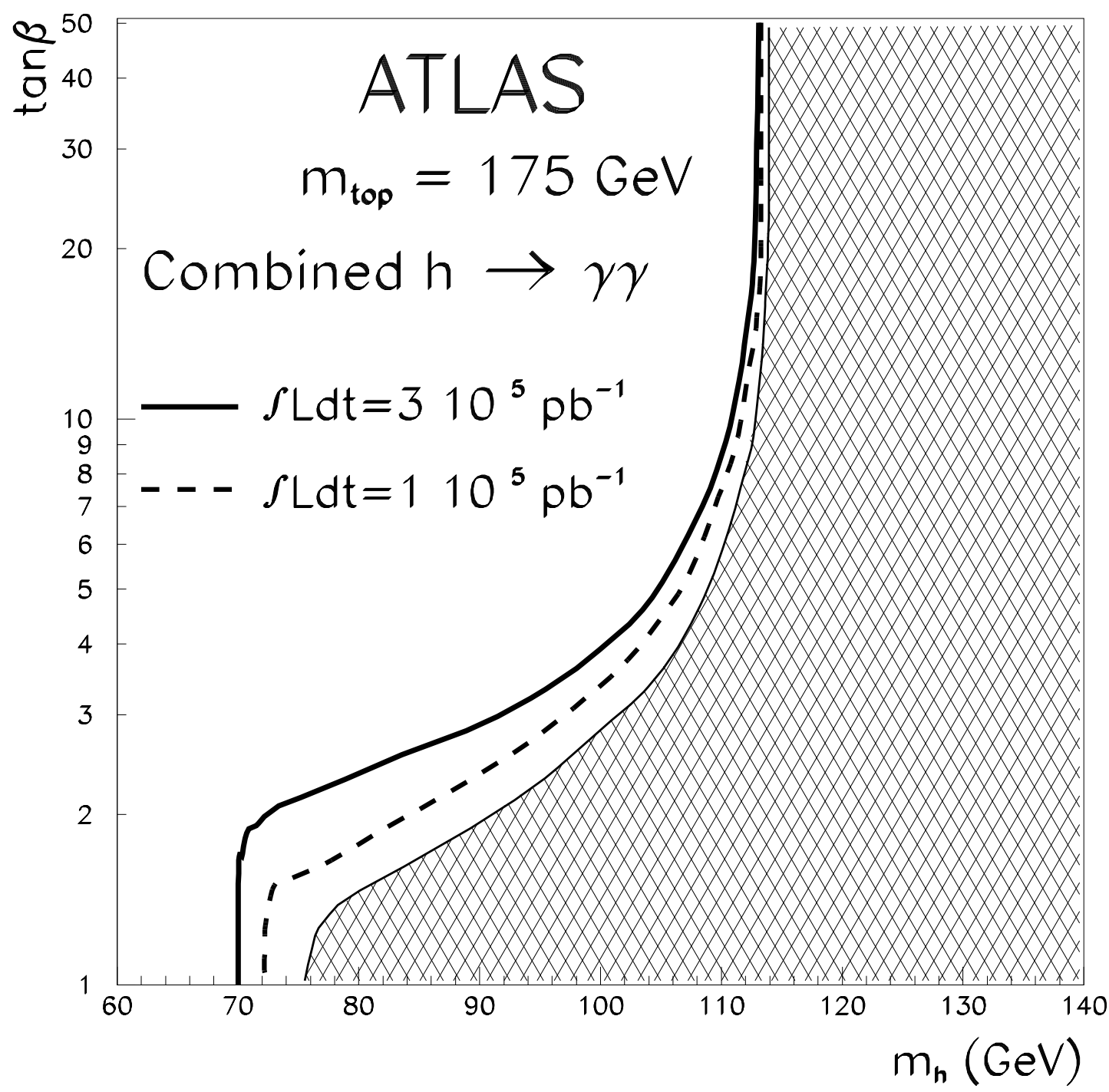

Figure 40: For $m_{\mathrm{t}}=175 \mathrm{GeV}$ and integrated luminosities of $10^{5} \mathrm{pb}^{-1}$ and $3 \cdot 10^{5} \mathrm{pb}^{-1}, 5 \sigma$-discovery contour curves for the combined $\mathrm{h} \rightarrow \gamma \gamma$ inclusive and associated channels in the $\left(m_{\mathrm{h}}, \tan \beta\right)$ plane. The cross-hatched area is theoretically excluded. 


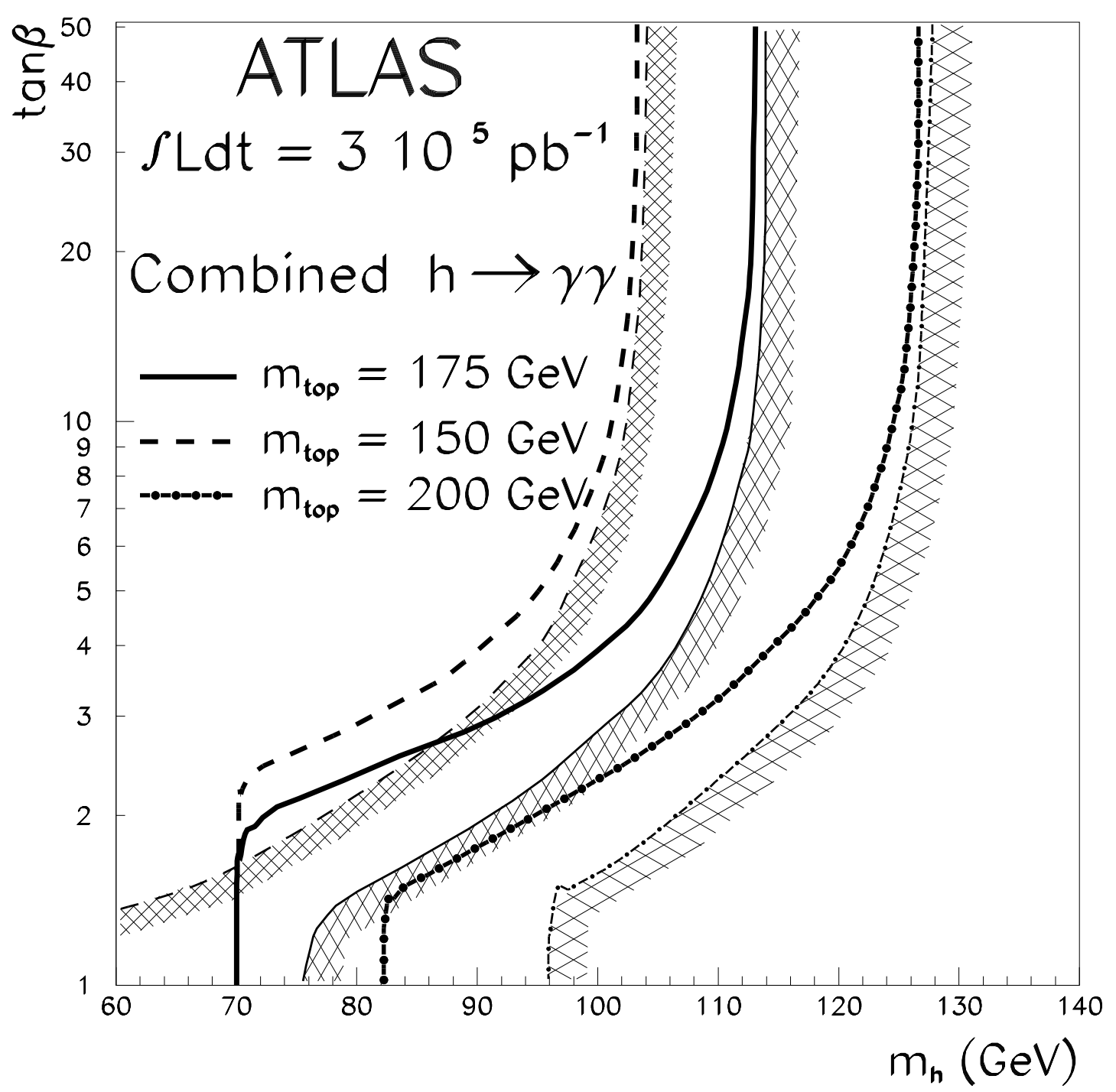

Figure 41: For $m_{\mathrm{t}}=150,175$ and $200 \mathrm{GeV}$ and an integrated luminosity of $3 \cdot 10^{5} \mathrm{pb}^{-1}, 5 \sigma$-discovery contour curves for the combined $\mathrm{h} \rightarrow \gamma \gamma$ inclusive and associated channels in the $\left(m_{\mathrm{h}}, \tan \beta\right)$ plane. The cross-hatched areas are theoretically excluded. 


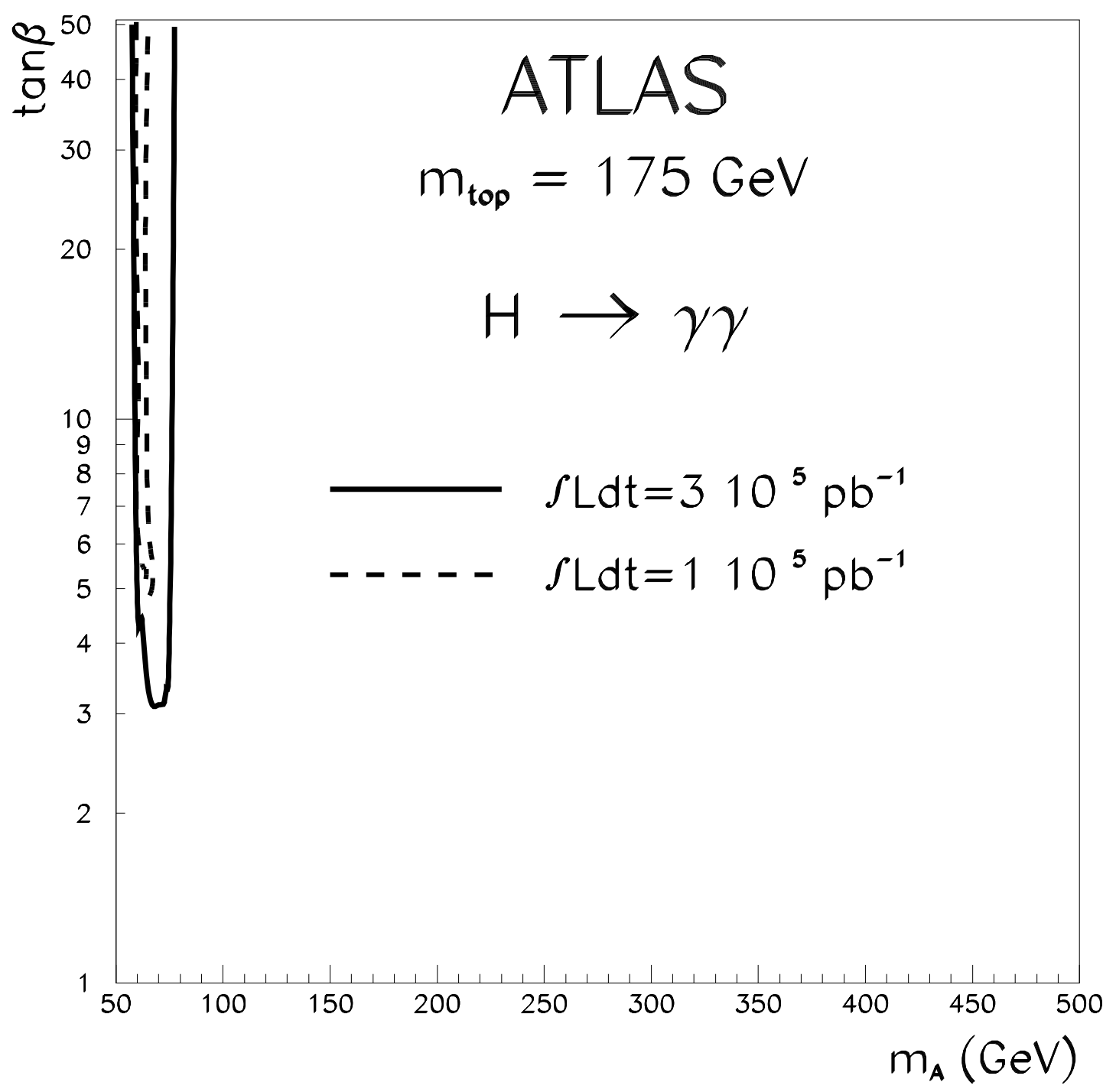

Figure 42: For $m_{\mathrm{t}}=175 \mathrm{GeV}$ and integrated luminosities of $10^{5} \mathrm{pb}^{-1}$ and $3 \cdot 10^{5} \mathrm{pb}^{-1}, 5 \sigma$-discovery contour curves for the $\mathrm{H} \rightarrow \gamma \gamma$ inclusive channel in the $\left(m_{\mathrm{A}}, \tan \beta\right)$ plane. 


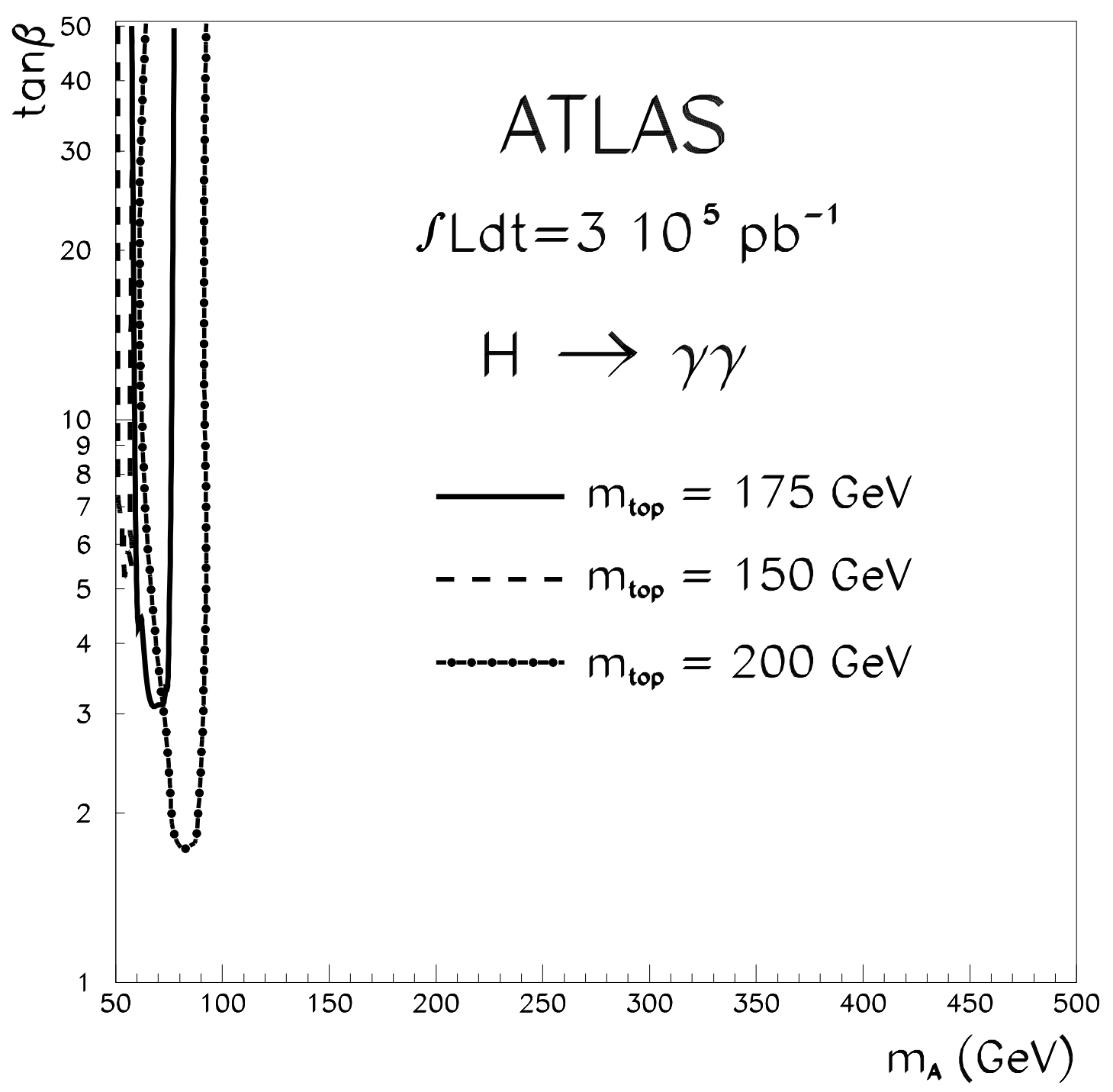

Figure 43: For $m_{\mathrm{t}}=150,175$ and $200 \mathrm{GeV}$ and an integrated luminosity of $3 \cdot 10^{5} \mathrm{pb}^{-1}, 5 \sigma$-discovery contour curves for the $\mathrm{H} \rightarrow \gamma \gamma$ inclusive channel in the $\left(m_{\mathrm{A}}, \tan \beta\right)$ plane. 


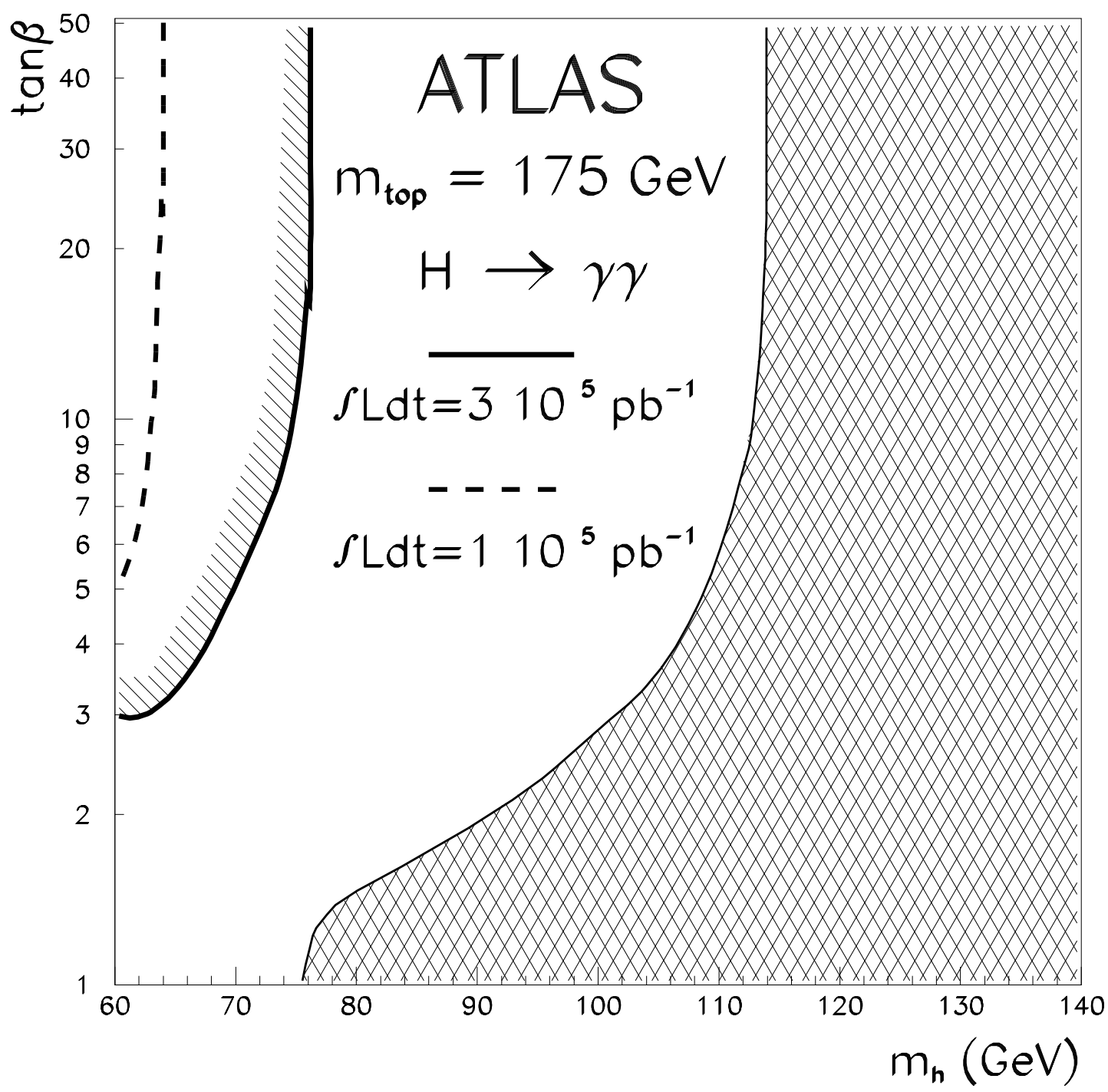

Figure 44: For $m_{\mathrm{t}}=175 \mathrm{GeV}$ and integrated luminosities of $10^{5} \mathrm{pb}^{-1}$ and $3 \cdot 10^{5} \mathrm{pb}^{-1}, 5 \sigma$-discovery contour curves for the $\mathrm{H} \rightarrow \gamma \gamma$ inclusive channel in the $\left(m_{\mathrm{h}}, \tan \beta\right)$ plane. The cross-hatched area is theoretically excluded. 


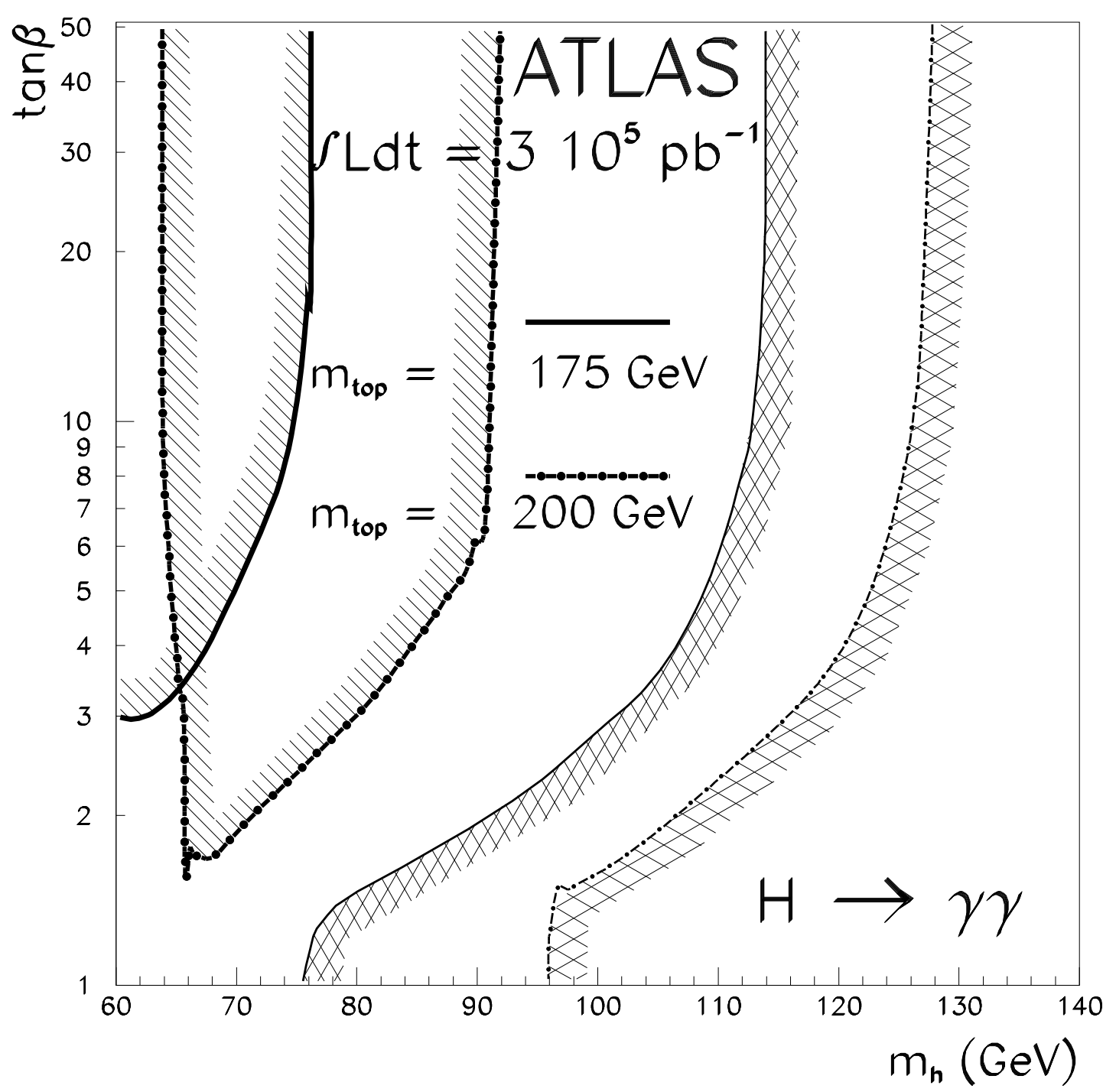

Figure 45: For $m_{\mathrm{t}}=175$ and $200 \mathrm{GeV}$ and an integrated luminosity of $3 \cdot 10^{5} \mathrm{pb}^{-1}, 5 \sigma$-discovery contour curves for the $\mathrm{H} \rightarrow \gamma \gamma$ inclusive channel in the $\left(m_{\mathrm{h}}, \tan \beta\right)$ plane. The cross-hatched areas are theoretically excluded. 


\subsection{The $\mathrm{h} \rightarrow \mathrm{b} \overline{\mathrm{b}}$ channel}

The SM H $\rightarrow b \bar{b}$ channel has recently been studied in [14], where both WH and $t \bar{t} \mathrm{H}$ production were considered, with final states containing one high$\mathrm{p}_{\mathrm{T}}$ lepton from $\mathrm{W}$-boson decay for triggering and two (resp. three or four) reconstructed b-jets in the WH (resp. $t \overline{\mathrm{t}} \mathrm{H}$ ) case.

The conclusions of [14] were that a signal from $H \rightarrow b \bar{b}$ decays may be observed above the background at the LHC for $m_{\mathrm{H}}<90-100 \mathrm{GeV}$ and an integrated luminosity of $3 \cdot 10^{4} \mathrm{pb}^{-1}$, provided excellent b-tagging performance can be achieved with the detector. Recent work on the b-tagging capabilities of the ATLAS detector and interactions with the physics referees can be summarised as follows:

- recent simulations [15], summarised in Appendix A, indicate that an overall b-tagging efficiency $\epsilon_{b}=60 \%$ can be achieved with the combined use of vertexing and of soft-lepton tags and with the B-layer present in the Inner Detector. The corresponding rejection expected against light-quark and gluon- (resp. charm-quark) jets is $\sim 100$ (resp. $\sim 10$ );

- a signal from WH production can only be seen above the dominant $t \bar{t}$ background if tight veto cuts against additional jets and leptons are applied [14], which is certainly possible at low luminosity. It is however clear that further studies are needed to define efficient veto cuts at high luminosity and understand whether the signal sensitivity can be improved;

- a signal from tÉ $\mathrm{H}$ production could probably not be extracted without a complete reconstruction of the top-quark decays to solve the large combinatorial problems arising from the presence of four b-quarks in the final state.

Therefore, until more work is done for the $t \bar{t} \mathrm{H}$ channel, the results of [14] are extrapolated to the MSSM Higgs sector by using only the WH channel at low luminosity and by rescaling the results of [14] to an integrated luminosity of $3 \cdot 10^{4} \mathrm{pb}^{-1}$ and to the expected improved b-tagging performance. Table 31 shows the resulting expected values for the signal and background rates, computed in a $\pm 20 \mathrm{GeV}$ mass bin around the reconstructed invariant mass of the $\mathrm{b} \overline{\mathrm{b}}$ pair, and the expected significances. The background from t $\overline{\mathrm{t}}$ production corresponds to $\sim 30 \%$ of the total background for $m_{\mathrm{t}}=175 \mathrm{GeV}$. For different values of $m_{\mathrm{t}}$, the change in production crosssection is more or less compensated for by the change in acceptance of the selection cuts, so both the signal and background rates are assumed here to be independent of $m_{\mathrm{t}}$. 
In the MSSM case, the rates are somewhat suppressed with respect to the $\mathrm{SM}$ case, as discussed in Section 3.1. As for the $\mathrm{h} \rightarrow \gamma \gamma$ channel, only values of $m_{\mathrm{h}}$ above $70 \mathrm{GeV}$ are considered, since the reconstruction efficiencies and background rates have not been studied for lower masses. The expected $5 \sigma$-discovery contour curves for an integrated luminosity of $3 \cdot 10^{4} \mathrm{pb}^{-1}$ are shown for $m_{\mathrm{t}}=150,175$ and $200 \mathrm{GeV}$ in Fig. 46 for the $\left(m_{\mathrm{A}}, \tan \beta\right)$ plane and in Fig. 47 for the $\left(m_{\mathrm{h}}, \tan \beta\right)$ plane. Although it provides limited coverage of the parameter space in the $\left(m_{\mathrm{A}}, \tan \beta\right)$ plane, especially for large values of $m_{\mathrm{t}}$, this channel is quite important, since it provides additional sensitivity with respect to the $h \rightarrow \gamma \gamma$ channel for low values of $\tan \beta$. Future work will determine whether any improvement in the sensitivity can be expected at high luminosity.

Table 31: Observability of the $S M \mathrm{H} \rightarrow \mathrm{b} \overline{\mathrm{b}}$ in associated production, $\mathrm{WH}$ with $\mathrm{W} \rightarrow \ell \nu$, for $m_{\mathrm{t}}=175 \mathrm{GeV}$ and for an integrated luminosity of $3 \cdot 10^{4} \mathrm{pb}^{-1}$. The numbers of signal and background events and the significances are extrapolated from Table 12 of [14], using $\epsilon_{\mathrm{b}}=60 \%$ and $R_{\text {jet }}=100$. The $\sigma \times B R$ values are taken from Table 1 of [14].

\begin{tabular}{|c|c||c|c|c|}
\hline \multicolumn{2}{|c||}{} & \multicolumn{3}{c|}{ Low luminosity } \\
\cline { 3 - 5 } \multicolumn{2}{|c|}{} & \multicolumn{3}{c|}{$3 \cdot 10^{4} \mathrm{pb}^{-1}$} \\
\hline $\begin{array}{c}m_{\mathrm{H}} \\
(\mathrm{GeV})\end{array}$ & $\begin{array}{c}\sigma \times B R \\
(\mathrm{fb})\end{array}$ & Signal & Background & Signif. \\
\hline \hline 80.0 & 769 & 756 & 16800 & 5.8 \\
100.0 & 405 & 475 & 12700 & 4.2 \\
120.0 & 209 & 203 & 9700 & 2.1 \\
\hline
\end{tabular}




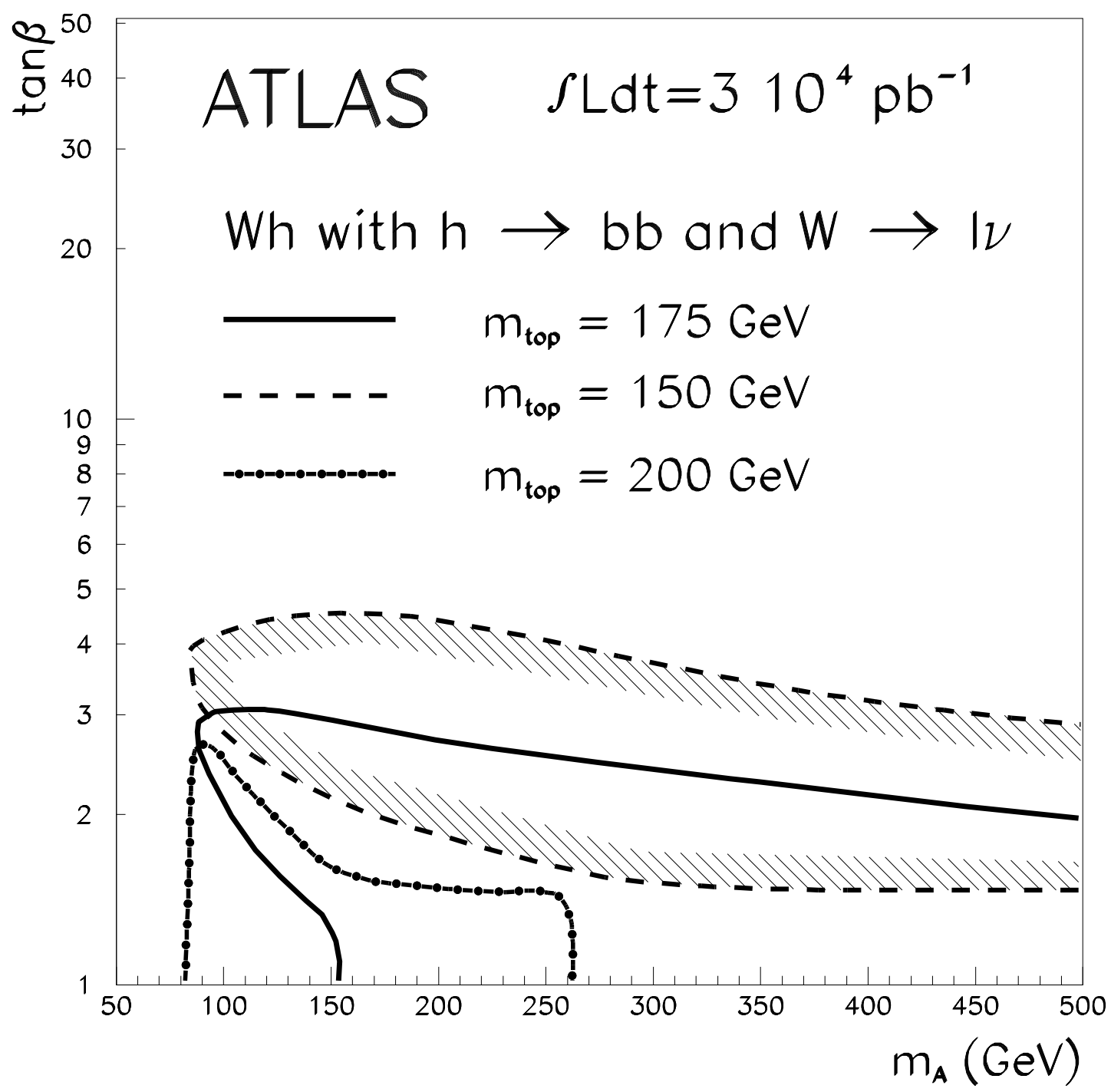

Figure 46: For $m_{\mathrm{t}}=150,175$ and $200 \mathrm{GeV}$ and an integrated luminosity of $3 \cdot 10^{4} \mathrm{pb}^{-1}, 5 \sigma$-discovery contour curves for the $\mathrm{h} \rightarrow \mathrm{b} \overline{\mathrm{b}}$ associated channel in the $\left(m_{\mathrm{A}}, \tan \beta\right)$ plane. 


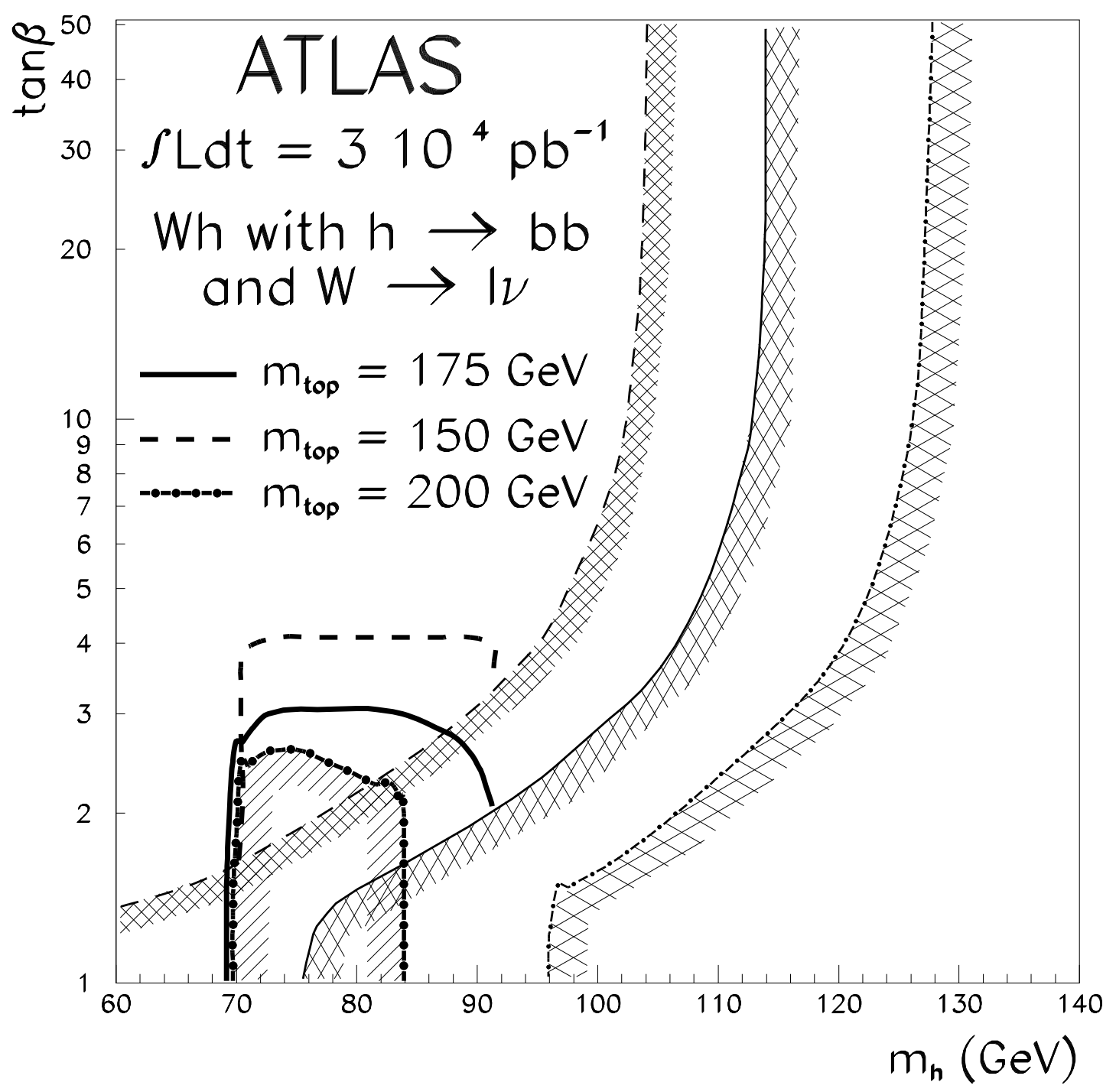

Figure 47: For $m_{\mathrm{t}}=150,175$ and $200 \mathrm{GeV}$ and an integrated luminosity of $3 \cdot 10^{4} \mathrm{pb}^{-1}, 5 \sigma$-discovery contour curves for the $\mathrm{h} \rightarrow \mathrm{b} \overline{\mathrm{b}}$ associated channel in the $\left(m_{\mathrm{h}}, \tan \beta\right)$ plane. The cross-hatched areas are theoretically excluded. 


\subsection{The $\mathrm{H} \rightarrow \mathrm{ZZ}^{(\star)} \rightarrow 4 \ell$ channel}

As for the $\mathrm{h} \rightarrow \gamma \gamma$ and $\mathrm{h} \rightarrow \mathrm{b} \overline{\mathrm{b}}$ channels, the observability in the MSSM of the $\mathrm{H} \rightarrow \mathrm{ZZ}^{(*)} \rightarrow 4 \ell$ channel is extrapolated from the detailed studies performed in the SM case, which are documented in the TP and in [3], but also in [16], [17] and [18] for more recent results.

For the intermediate mass range, $120<m_{\mathrm{H}}<2 m_{\mathrm{Z}}$, the signal rates are small and the background rates are potentially very large. In particular, the reducible $t \bar{t}$ and $Z b \bar{b}$ backgrounds can only be brought down to a level well below the irreducible $\mathrm{ZZ}^{*} / \gamma^{*}$ background by a combination of strong isolation and impact parameter cuts. For this reason, the overall signal reconstruction efficiency is $\sim 40 \%$ at low luminosity, corresponding to a reconstruction efficiency of $90 \%$ per lepton, an efficiency of $85 \%$ for the lepton isolation cuts, an efficiency of $85 \%$ for the impact parameter cuts, an efficiency of $95 \%$ for the four-lepton mass reconstruction in the chosen mass bin, and an efficiency of $90 \%$ for losses due to internal bremsstrahlung [17], which were not included in the TP. This overall efficiency of $40 \%$ drops to $24 \%$ at high luminosity, due to the lower efficiency of the lepton isolation cuts. Table 32 gives updated estimates at low and high luminosity for the signal and background rates as well as for the expected significances, which were computed as discussed in Section 4.

For the range of masses accessible in the MSSM case above the ZZ threshold, $2 m_{\mathrm{Z}}<m_{\mathrm{H}}<400 \mathrm{GeV}$ (see Section 3.2), the only significant background arises from irreducible $\mathrm{ZZ}$ continuum production. The overall signal reconstruction efficiency is thus significantly higher, $\sim 59 \%$, corresponding to a reconstruction efficiency of $90 \%$ per lepton and an efficiency of $90 \%$ for the four-lepton mass reconstruction within the chosen mass bin. In the SM case, for which the Higgs-boson width increases rapidly as $m_{\mathrm{H}}$ increases, this mass bin was chosen to be $m_{\mathrm{H}} \pm 1.64 \sqrt{\left(\Gamma_{\mathrm{H}}^{\mathrm{tot}} / 2.36\right)^{2}+\sigma_{m}^{2}}$, where $\sigma_{m}$ is the expected experimental mass resolution [3]. Since, however, the MSSM H-boson width remains much narrower than the experimental resolution over the relevant region of parameter space (see Section 2.6), the mass bin chosen for the MSSM case is narrower, $m_{\mathrm{H}} \pm 1.64 \sigma_{m}$, where $\sigma_{m} / m_{\mathrm{H}} \sim 1.5 \%$ was estimated from recent studies using full simulation for the $H \rightarrow 4 e$ channel and from updated detailed parametrisations of the overall muon momentum resolution for the $H \rightarrow 4 \mu$ channel [18]. The expected signal and background rates and the expected significances at low and high luminosity are given in Table 33, extrapolated from Table 38 of [3].

As shown in some detail in Section 3.2 , the MSSM H $\rightarrow \mathrm{ZZ}^{(\star)} \rightarrow 4 \ell$ rates are strongly suppressed with respect to the SM case (except for values of $\tan \beta$ smaller than unity). This limits the observability of this channel to $m_{\mathrm{H}}<2 m_{\mathrm{t}}$ and to low values of $\tan \beta$. The expected $5 \sigma$-discovery contour curves are shown in Figs. 48 to 51 in the usual way. The highest possible integrated luminosity is needed in this channel and the observability of the signal depends strongly on the value of $m_{\mathrm{t}}$, as shown in Figs. 49 and 51. 
If a signal were to be observed in this channel, the measured signal rate would provide the best tool to understand its origin, since the $\mathrm{H} \rightarrow \mathrm{ZZ}^{(\star)} \rightarrow 4 \ell$ MSSM rates are suppressed by an order of magnitude with respect to the SM case over most of the parameter space, and would allow a measurement of the value of $\tan \beta$ with an accuracy of \pm 10 to $15 \%$, for an integrated luminosity of $3 \cdot 10^{5} \mathrm{pb}^{-1}$. For values of $m_{\mathrm{H}}$ larger than $\sim 250 \mathrm{GeV}$, the measured signal width would also provide a handle to disentangle the SM case $\left(\Gamma_{\mathrm{H}}^{\mathrm{tot}} \sim 10 \mathrm{GeV}\right)$ from the MSSM case $\left(\Gamma_{\mathrm{H}}^{\mathrm{tot}}<1 \mathrm{GeV}\right)$.

Table 32: Observability of the $S M \mathrm{H} \rightarrow \mathrm{ZZ}^{\star}$ channel at low and high luminosities. The expected numbers of signal and background events are extrapolated from Table 29 of [3].

\begin{tabular}{|c|c||c|c|c||c|c|c|}
\hline \multicolumn{2}{|c||}{} & \multicolumn{3}{c||}{ Low luminosity } & \multicolumn{3}{c|}{ High luminosity } \\
\cline { 2 - 7 } \multicolumn{2}{|c||}{} & \multicolumn{3}{c||}{$3 \cdot 10^{4} \mathrm{pb}^{-1}$} & \multicolumn{3}{c|}{$\mathrm{pb}^{-1}$} \\
\hline$m_{\mathrm{H}}$ & $\begin{array}{c}\sigma \times B R \\
(\mathrm{GeV})\end{array}$ & Signal & Background & Signif. & Signal & Background & Signif. \\
\hline \hline 120.0 & 1.36 & 2.3 & 1.7 & 1.0 & 4.7 & 4.7 & 1.6 \\
130.0 & 3.12 & 11.2 & 3.0 & 4.5 & 22.0 & 8.2 & 7.7 \\
150.0 & 5.73 & 31.4 & 3.6 & 16.5 & 61.6 & 10.0 & 19.5 \\
170.0 & 1.44 & 9.2 & 3.7 & 3.31 & 17.9 & 9.5 & 4.6 \\
180.0 & 3.33 & 24.0 & 3.6 & 7.8 & 46.7 & 9.0 & 15.6 \\
\hline
\end{tabular}

Table 33: Observability of the $S M \mathrm{H} \rightarrow \mathrm{ZZ} \rightarrow 4 \ell$ channel at low and high luminosities. The expected numbers of signal and background events are extrapolated from Table 38 of [3].

\begin{tabular}{|c|c||c|c|c||c|c|c|}
\hline \multicolumn{2}{|c||}{} & \multicolumn{3}{c||}{ Low luminosity } & \multicolumn{3}{c|}{ High luminosity } \\
\cline { 3 - 7 } \multicolumn{2}{|c||}{} & \multicolumn{3}{c||}{$3 \cdot 10^{4} \mathrm{pb}^{-1}$} & \multicolumn{3}{c|}{$\mathrm{pb}^{-1}$} \\
\hline$m_{\mathrm{H}}$ & $\begin{array}{c}\sigma \times B R \\
(\mathrm{GeV})\end{array}$ & Signal & Background & Signif. & Signal & Background & Signif. \\
\hline \hline 200.0 & 12.4 & 56.0 & 5.34 & 24.2 & 186.0 & 17.8 & 44.1 \\
220.0 & 11.9 & 27.0 & 0.87 & 28.9 & 89.0 & 2.9 & 52.3 \\
240.0 & 11.2 & 33.0 & 1.08 & 31.8 & 109.0 & 3.6 & 57.4 \\
260.0 & 10.3 & 35.0 & 0.84 & 38.2 & 115.0 & 2.8 & 68.7 \\
280.0 & 9.6 & 38.0 & 1.20 & 34.7 & 126.0 & 4.0 & 63.0 \\
300.0 & 9.1 & 39.0 & 1.17 & 36.1 & 130.0 & 3.9 & 65.8 \\
320.0 & 8.9 & 40.0 & 1.11 & 38.0 & 134.0 & 3.7 & 69.7 \\
340.0 & 9.0 & 42.0 & 1.02 & 41.6 & 139.0 & 3.4 & 75.4 \\
360.0 & 8.6 & 44.0 & 0.63 & 55.4 & 147.0 & 2.1 & 101.4 \\
380.0 & 7.7 & 39.0 & 0.84 & 42.6 & 131.0 & 2.8 & 78.3 \\
400.0 & 6.7 & 38.0 & 0.87 & 40.7 & 126.0 & 2.9 & 74.0 \\
\hline
\end{tabular}




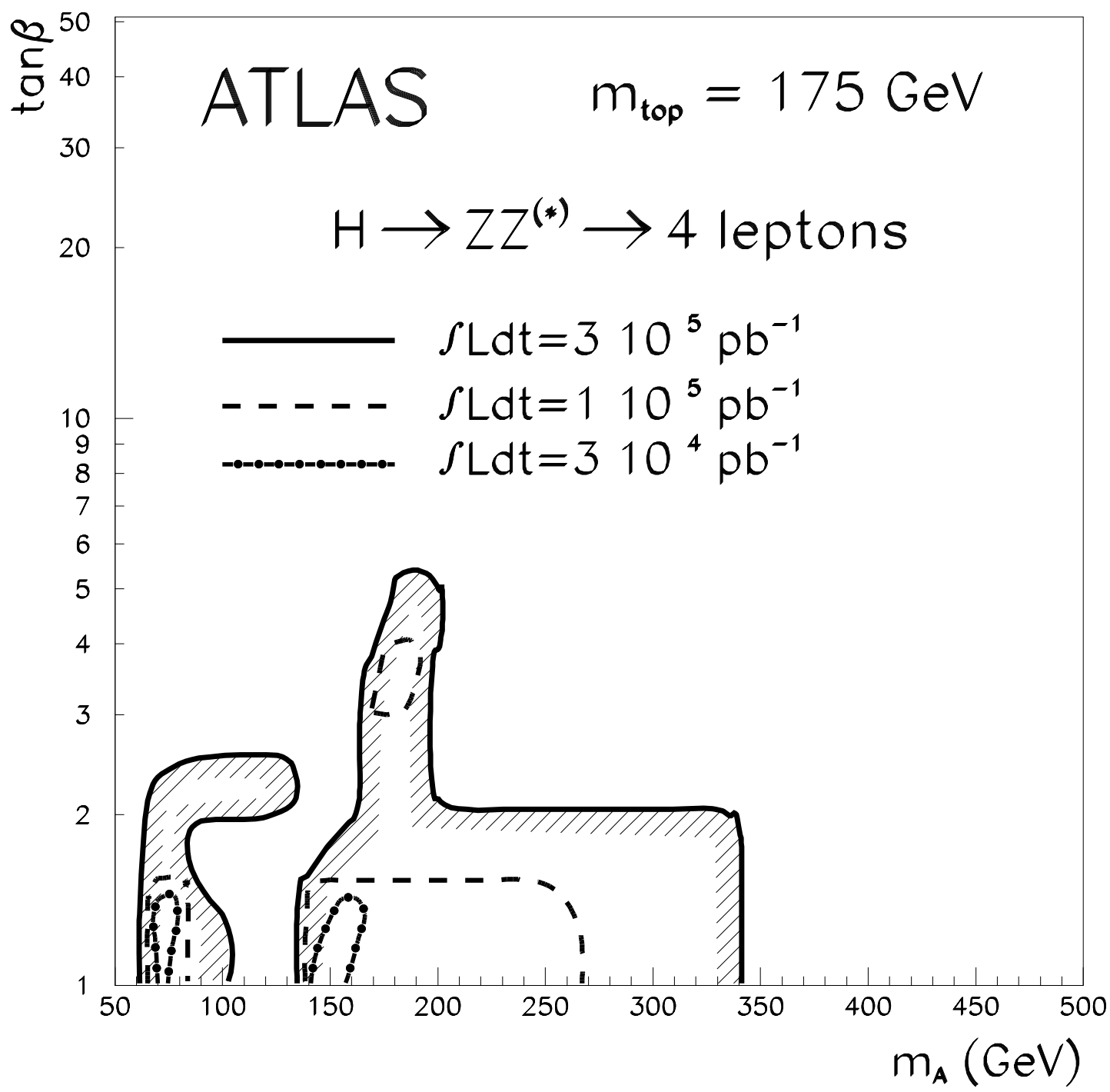

Figure 48: For $m_{\mathrm{t}}=175 \mathrm{GeV}$ and integrated luminosities of $3 \cdot 10^{4} \mathrm{pb}^{-1}$, $10^{5} \mathrm{pb}^{-1}$ and $3 \cdot 10^{5} \mathrm{pb}^{-1}, 5 \sigma$-discovery contour curves for the $\mathrm{H} \rightarrow \mathrm{ZZ}^{(\star)} \rightarrow 4 \ell$ channel in the $\left(m_{\mathrm{A}}, \tan \beta\right)$ plane. 


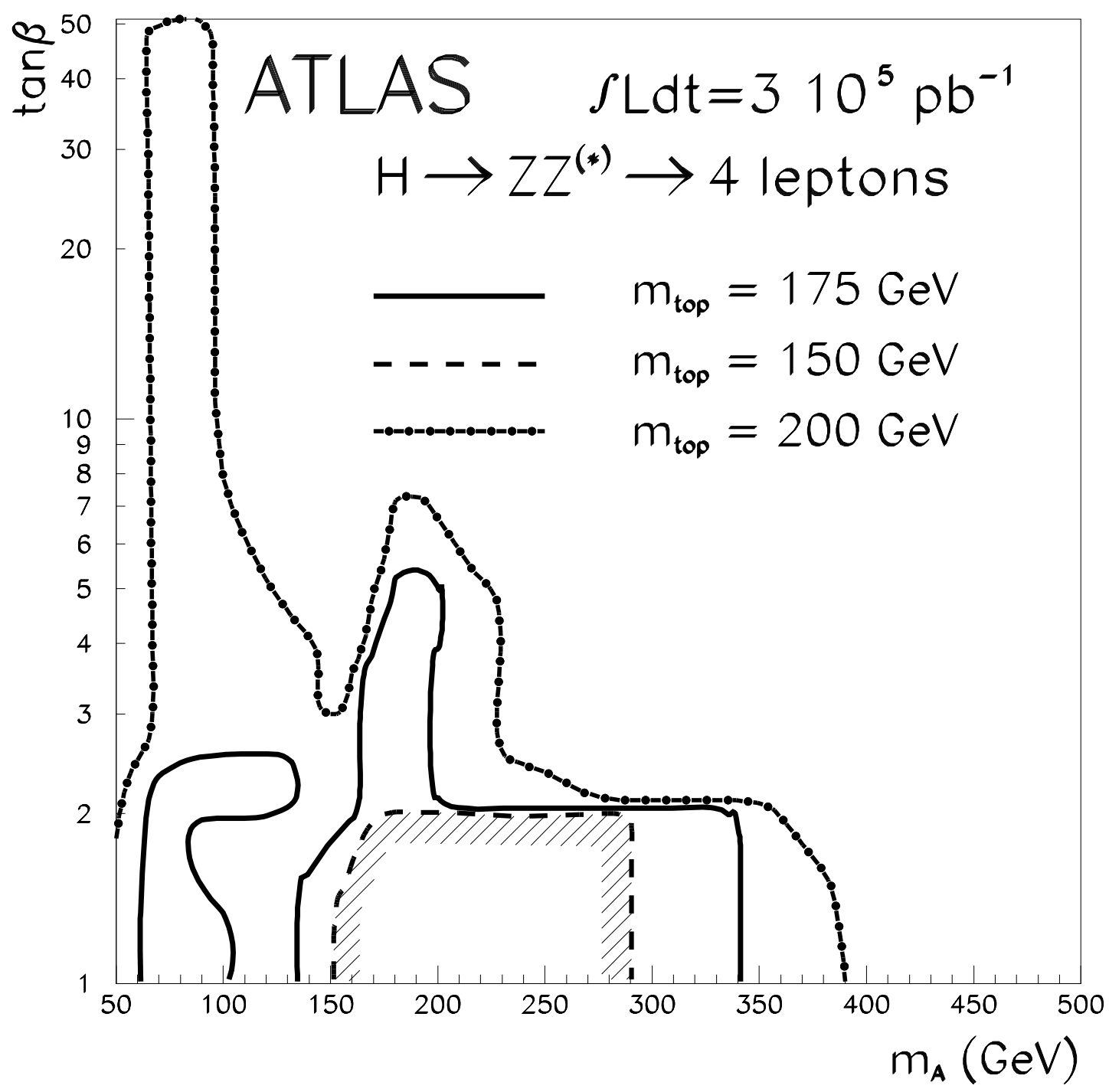

Figure 49: For $m_{\mathrm{t}}=150,175$ and $200 \mathrm{GeV}$ and an integrated luminosity of $3 \cdot 10^{5} \mathrm{pb}^{-1}, 5 \sigma$-discovery contour curves for the $\mathrm{H} \rightarrow \mathrm{ZZ}^{(\star)} \rightarrow 4 \ell$ channel in the $\left(m_{\mathrm{A}}, \tan \beta\right)$ plane. 


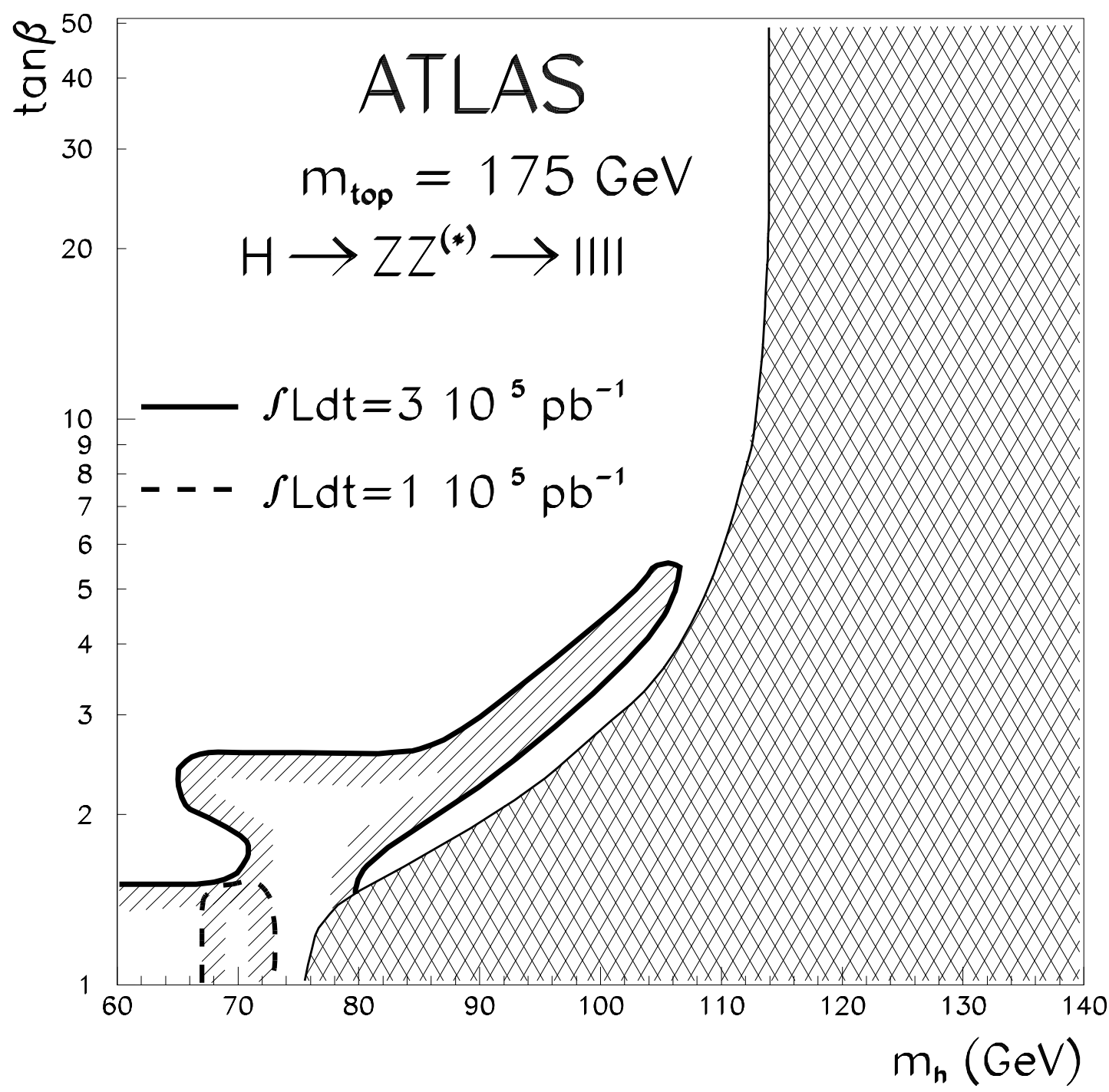

Figure 50: For $m_{\mathrm{t}}=175 \mathrm{GeV}$ and integrated luminosities of $10^{5} \mathrm{pb}^{-1}$ and $3 \cdot 10^{5} \mathrm{pb}^{-1}, 5 \sigma$-discovery contour curves for the $\mathrm{H} \rightarrow \mathrm{ZZ}^{(\star)} \rightarrow 4 \ell$ channel in the $\left(m_{\mathrm{h}}, \tan \beta\right)$ plane. The cross-hatched area is theoretically excluded. 


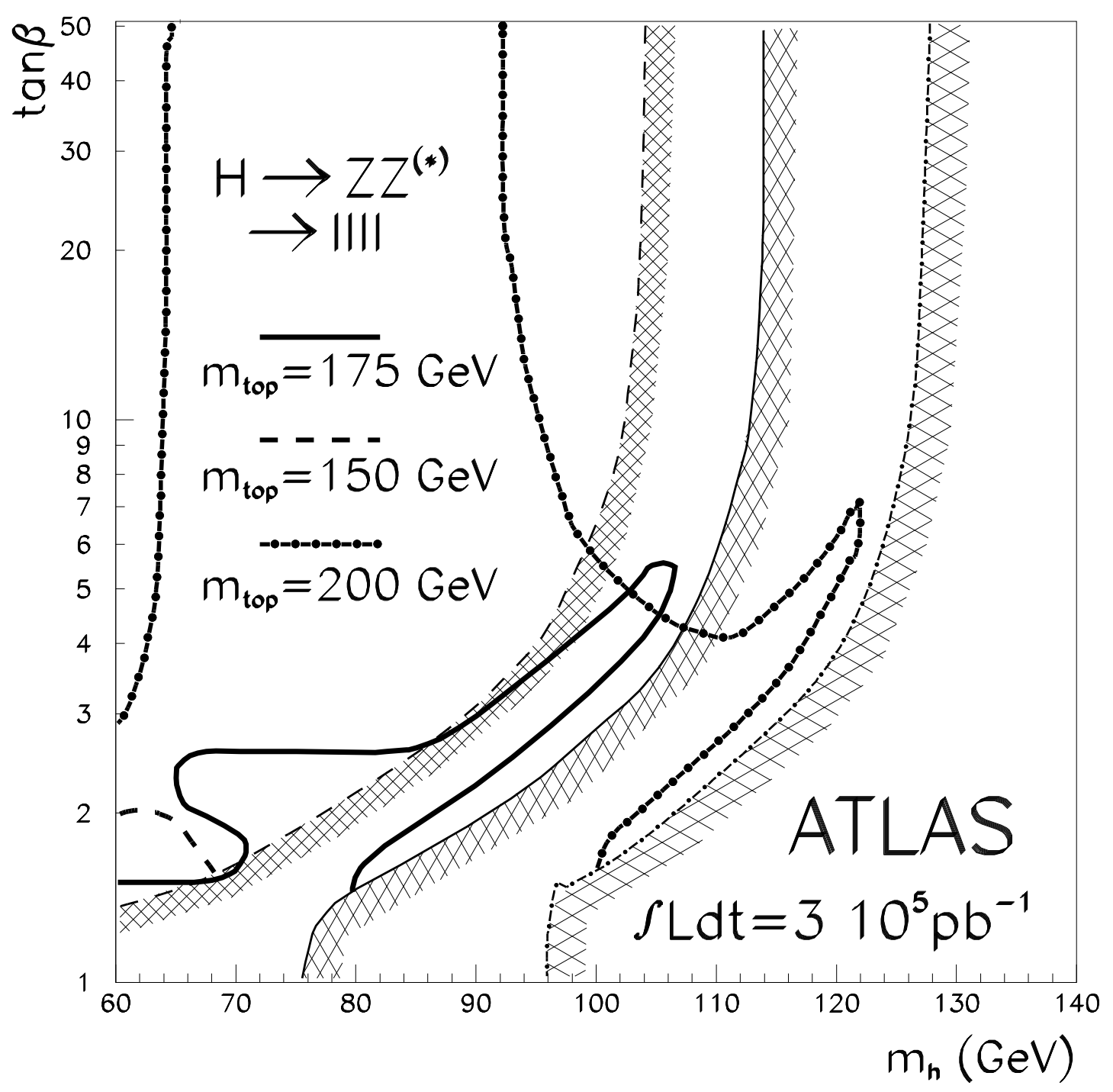

Figure 51: For $m_{\mathrm{t}}=150,175$ and $200 \mathrm{GeV}$ and an integrated luminosity of $3 \cdot 10^{5} \mathrm{pb}^{-1}, 5 \sigma$-discovery contour curves for the $\mathrm{H} \rightarrow \mathrm{ZZ}^{(\star)} \rightarrow 4 \ell$ channel in the $\left(m_{\mathrm{h}}, \tan \beta\right)$ plane. The cross-hatched areas are theoretically excluded. 


\subsection{The $\mathrm{H} / \mathrm{A} \rightarrow \tau \tau$ channel}

In the $\mathrm{SM}$ case, a signal from $\mathrm{H} \rightarrow \tau \tau$ decays cannot be observed experimentally at the LHC because the signal rates are too low with respect to the large backgrounds [19]. However, as explained in Sections 3.4 and 3.5, the MSSM $\mathrm{H} \rightarrow \tau \tau$ and $\mathrm{A} \rightarrow \tau \tau$ rates are strongly enhanced with respect to the $\mathrm{SM}$ case over a large region of the parameter space. For low values of $\tan \beta$, the $g g \rightarrow A, \mathrm{~A} \rightarrow \tau \tau$ rates are dominant and significantly larger than in the $\mathrm{SM}$ case. For large values of $\tan \beta$, the production is dominated by $\mathrm{b} \overline{\mathrm{b}} \mathrm{H}$ and $\mathrm{b} \overline{\mathrm{b}} \mathrm{A}$, and the $\mathrm{H} \rightarrow \tau \tau$ rates are very similar to the $\mathrm{A} \rightarrow \tau \tau$ ones. As discussed below, for $m_{\mathrm{A}}>150 \mathrm{GeV}$, the $\mathrm{H}$ - and A-bosons are degenerate in mass (see Section 2), so the signal rates in the $\tau \tau$ channel can be added, whereas a more complicated procedure depending on the experimental resolution and on the mass difference $m_{\mathrm{H}}-m_{\mathrm{A}}$ has to be applied for $m_{\mathrm{A}}<150 \mathrm{GeV}$. Higgs boson masses below $100 \mathrm{GeV}$ have not been considered in this channel because of the large resonant background from $\mathrm{Z} \rightarrow \tau \tau$ decays.

As discussed in the TP and in the detailed studies reported in [20], this channel requires excellent $\tau$ identification to suppress the huge QCD-jet backgrounds from various sources, but also excellent $E_{T}^{\text {miss }}$ resolution [21] for the reconstruction of the $\tau \tau$ invariant mass. One of the $\tau$-leptons is required to decay leptonically to trigger the experiment. The other $\tau$-lepton is then required to decay either to another lepton (lepton-lepton channel) or to a single charged hadron (lepton-hadron channel). The lepton-hadron channel turns out to provide the best sensitivity to a possible signal, due both to its larger rate and to the more favourable kinematics of the $\tau$-decay.

The background, a mixture of $t \bar{t}, b \bar{b}, W+$ jets and $\mathrm{Z}$, can be significantly reduced by appropriate kinematic cuts based on the reconstructed lepton, on the $\tau$-jet and on $E_{T}^{\text {miss }}$. After all cuts, t't decays amount to only 10 to $20 \%$ of the total background, which is dominated by $W+$ jet and $b \bar{b}$ events (and Z-decays for the lower values of $m_{\mathrm{H}}$ and $m_{\mathrm{A}}$ ). Therefore, the background estimates in this channel were assumed to be independent of $m_{\mathrm{t}}$, since the smaller $t \bar{t}$ cross-section is more or less compensated for by the larger acceptance of the selection cuts as $m_{\mathrm{t}}$ increases.

The full simulation studies of the expected $\tau \tau$ mass resolution as a function of $m_{\mathrm{H}}$ and $m_{\mathrm{A}}[20]$ have since been extended to lower (100 and $120 \mathrm{GeV}$ ) and higher $(400$ and $500 \mathrm{GeV})$ mass values. The mass resolution increases from $12 \mathrm{GeV}$ to $50 \mathrm{GeV}$ if the Higgs-boson mass increases from $100 \mathrm{GeV}$ to $500 \mathrm{GeV}$. Table 34 shows the expected signal and background rates as a function of $m_{\mathrm{A}}$, for $\mathrm{A} \rightarrow \tau \tau$ decays and for $\tan \beta=10$, as well as the expected significances at low and high luminosity. These numbers have been obtained from full simulation and reconstruction of $g g \rightarrow A \rightarrow \tau \tau$ decays. Since the dominant production process for large values of $\tan \beta$ in this channel is from $\mathrm{b} \overline{\mathrm{b}} \mathrm{A}$ production, the acceptance and reconstruction efficiency for this subprocess are presently also under study. The results of this study 
may decrease somewhat the sensitivity in this channel due to the smaller average $\mathrm{p}_{\mathrm{T}}$ of the produced A-boson.

At high luminosity, although the $\tau$ identification efficiency can be maintained at its low-luminosity value of $\sim 26 \%$, the sensitivity to this channel is significantly degraded due to pile-up effects for the following two main reasons:

- the fraction of cases where the neutrino system can be resolved [19] decreases by $30 \%$;

- the $\tau \tau$ mass resolution is degraded by a factor $\sim 1.5$.

As a consequence, high-luminosity operation with $10^{5} \mathrm{pb}^{-1}$ is expected to only slightly improve the sensitivity to a possible signal with respect to lowluminosity operation with $3 \cdot 10^{4} \mathrm{pb}^{-1}$, as shown in Table 34 .

The significances obtained from Table 34 for the A-boson are then combined with those expected for the H-boson. For $m_{\mathrm{A}}>150 \mathrm{GeV}$, the significance values of Table 34 are approximately doubled since the $H \rightarrow \tau \tau$ and $\mathrm{A} \rightarrow \tau \tau$ rates are very similar and the two bosons are degenerate in mass. For $m_{\mathrm{A}}<150 \mathrm{GeV}$, the $\mathrm{H}-$ and A-boson masses can no longer be considered to be degenerate with respect to the experimental resolution and the combined significance for both channels is estimated as

$$
\sqrt{(S / \sqrt{B})_{A}^{2}+(S / \sqrt{B})_{H}^{2}-2 \epsilon(S / \sqrt{B})_{A}(S / \sqrt{B})_{H}},
$$

where $\epsilon$ was estimated from the results of the full simulation to be $\epsilon=-$ 0.33 for $\left|m_{\mathrm{H}}-m_{\mathrm{A}}\right| / \sigma_{m}=1.4$ (obviously $\epsilon \sim-1$ for $\left|m_{\mathrm{H}}-m_{\mathrm{A}}\right| / \sigma_{m} \sim 0$ and $\epsilon \sim 0$ for $\left.\left|m_{\mathrm{H}}-m_{\mathrm{A}}\right| / \sigma_{m} \gg 2\right)$.

The expected $5 \sigma$-discovery contour curves for the combined $\mathrm{H} / \mathrm{A} \rightarrow \tau \tau$ signal are shown in Figs. 52 to 55 . Fig. 52 shows that, even for a moderate integrated luminosity of $3 \cdot 10^{4} \mathrm{pb}^{-1}$, a signal should be observed over a large region of the $\left(m_{\mathrm{A}}, \tan \beta\right)$ plane. This region can be substantially increased only for the largest integrated luminosities achievable with high luminosity operation, due to the degraded detector performance at high luminosity discussed above. Fig. 53 shows that the observability of this channel does not vary much as a function of $m_{\mathrm{t}}$. As already mentioned, for low values of $\tan \beta$, the signal can be observed only in the $\mathrm{A} \rightarrow \tau \tau$ channel, and the sensitivity to the signal disappears for $m_{\mathrm{A}}>2 m_{\mathrm{t}}$, where $\mathrm{A} \rightarrow \mathrm{t} \overline{\mathrm{t}}$ decays become dominant. The shapes of the $5 \sigma$-discovery contour curves as a function of $m_{\mathrm{t}}$ shown in Fig. 55 reflect the variation in the relationship between $m_{\mathrm{A}}$ and $m_{\mathrm{h}}$ as a function of $m_{\mathrm{t}}$. 
Finally, it should be noted that, as in the case of $\mathrm{H} \rightarrow \mathrm{ZZ}^{(\star)} \rightarrow 4 \ell$ decays, a measurement of the signal rate should provide good sensitivity to $\tan \beta$ in this channel. As an example, for $m_{\mathrm{A}}=150 \mathrm{GeV}$ and an integrated luminosity of $3 \cdot 10^{5} \mathrm{pb}^{-1}, \tan \beta$ can be measured to an accuracy of $\pm 5 \%$ for $\tan \beta=5$ and of $\pm 13 \%$ for $\tan \beta=40$ (a systematic uncertainty of $\pm 10 \%$ was assumed for the measured signal rate).

Table 34: Observability of the $\mathrm{A} \rightarrow \tau \tau$ channel at low and high luminosities. The $\sigma \times B R$ values and the expected numbers of signal and background events are given for $\mathrm{A} \rightarrow \tau \tau$ decays and for $\tan \beta=10$.

\begin{tabular}{|c|c||c|c|c||c|c|c|}
\hline \multicolumn{2}{|c||}{} & \multicolumn{3}{c||}{ Low luminosity } & \multicolumn{3}{c|}{ High luminosity } \\
\cline { 2 - 7 } & \multicolumn{3}{c|}{$3 \cdot 10^{4} \mathrm{pb}^{-1}$} & & \\
\hline$m_{\mathrm{A}}$ & $\begin{array}{c}\sigma \times B R \\
(\mathrm{pb})\end{array}$ & Signal & Background & Signif. & Signal & Background & Signif. \\
\hline $\mathrm{GeV})$ & & & & & & \\
\hline 100.0 & 9.13 & 77 & 810 & 2.7 & 180 & 4050 & 2.8 \\
120.0 & 4.60 & 114 & 500 & 5.1 & 266 & 2500 & 5.3 \\
140.0 & 2.91 & 195 & 600 & 8.0 & 455 & 3000 & 8.3 \\
150.0 & 1.97 & 143 & 660 & 5.6 & 334 & 3300 & 5.8 \\
200.0 & 0.74 & 134 & 540 & 5.8 & 313 & 2700 & 6.0 \\
300.0 & 0.15 & 38 & 530 & 1.7 & 89 & 2650 & 1.7 \\
400.0 & 0.05 & 18 & 270 & 1.1 & 42 & 1350 & 1.1 \\
500.0 & 0.02 & 4 & 260 & 0.2 & 9 & 1300 & 0.2 \\
\hline
\end{tabular}




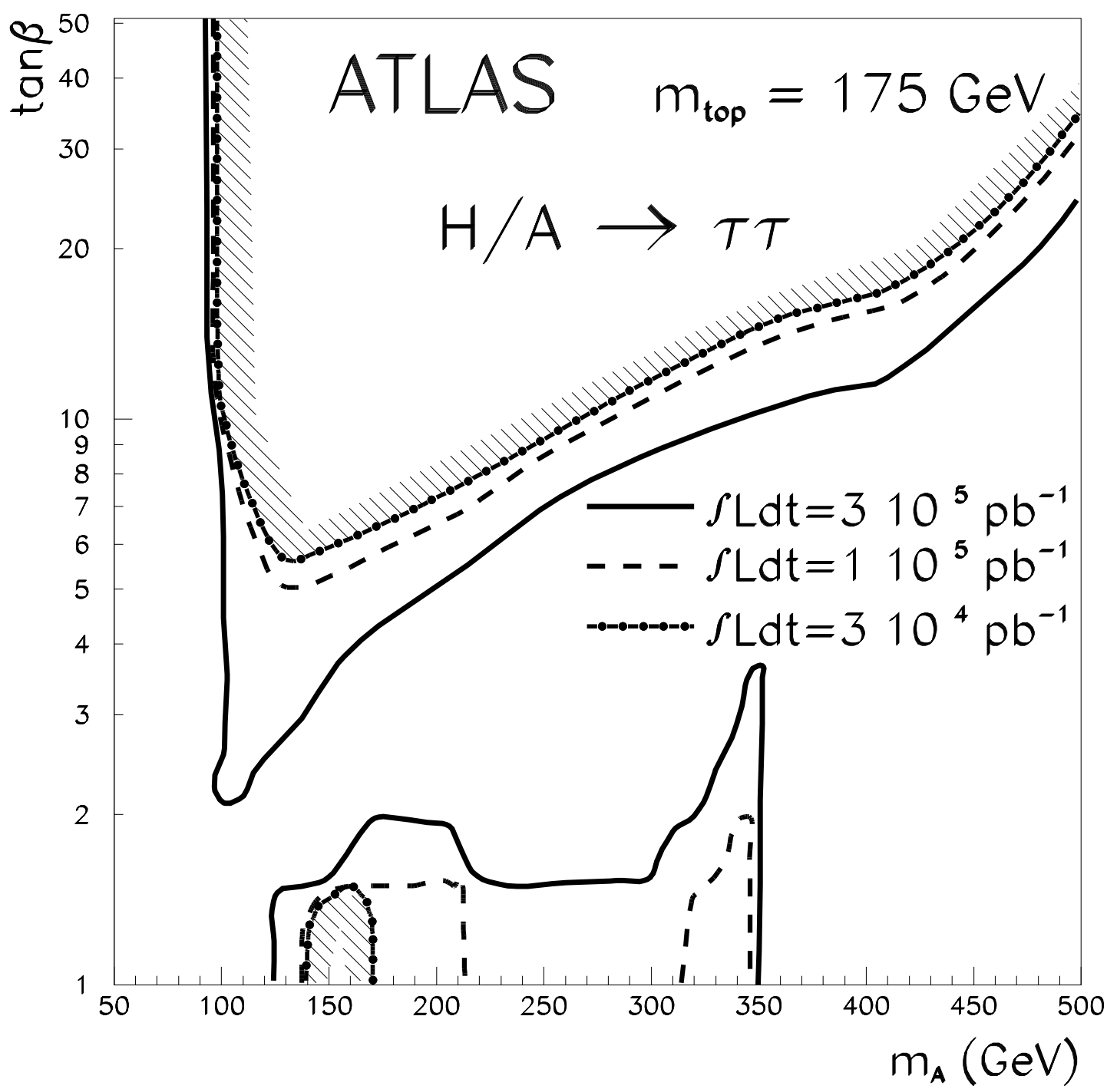

Figure 52: For $m_{\mathrm{t}}=175 \mathrm{GeV}$ and integrated luminosities of $3 \cdot 10^{4} \mathrm{pb}^{-1}$, $10^{5} \mathrm{pb}^{-1}$ and $3 \cdot 10^{5} \mathrm{pb}^{-1}, 5 \sigma$-discovery contour curves for the combined $\mathrm{H} / \mathrm{A} \rightarrow \tau \tau$ channel in the $\left(m_{\mathrm{A}}, \tan \beta\right)$ plane. 


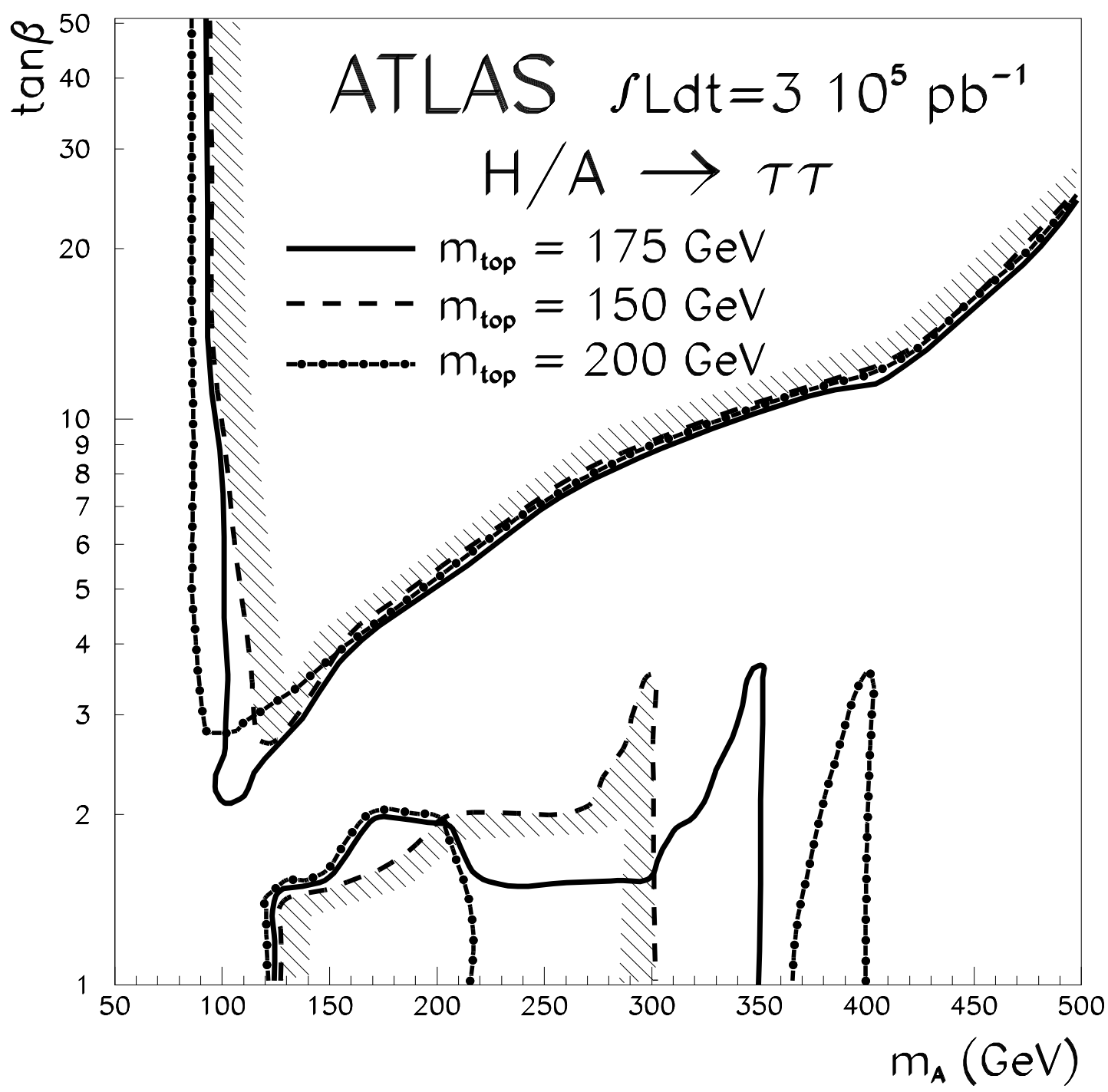

Figure 53: For $m_{\mathrm{t}}=150,175$ and $200 \mathrm{GeV}$ and an integrated luminosity of $3 \cdot 10^{5} \mathrm{pb}^{-1}, 5 \sigma$-discovery contour curves for the combined $\mathrm{H} / \mathrm{A} \rightarrow \tau \tau$ channel in the $\left(m_{\mathrm{A}}, \tan \beta\right)$ plane. 


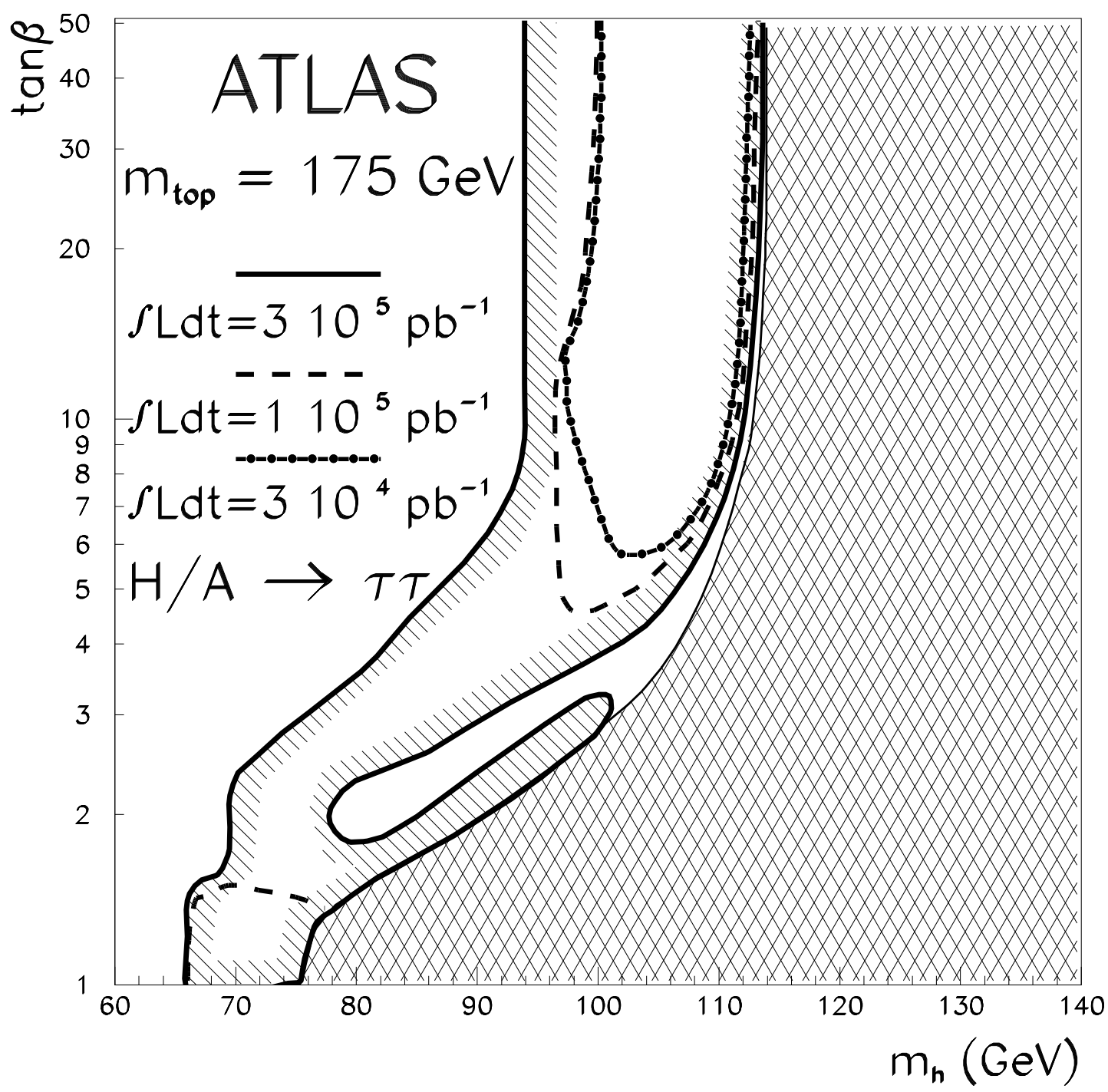

Figure 54: For $m_{\mathrm{t}}=175 \mathrm{GeV}$ and integrated luminosities of $3 \cdot 10^{4} \mathrm{pb}^{-1}$, $10^{5} \mathrm{pb}^{-1}$ and $3 \cdot 10^{5} \mathrm{pb}^{-1}, 5 \sigma$-discovery contour curves for the combined $\mathrm{H} / \mathrm{A} \rightarrow \tau \tau$ channel in the $\left(m_{\mathrm{h}}, \tan \beta\right)$ plane. The cross-hatched area is theoretically excluded. 


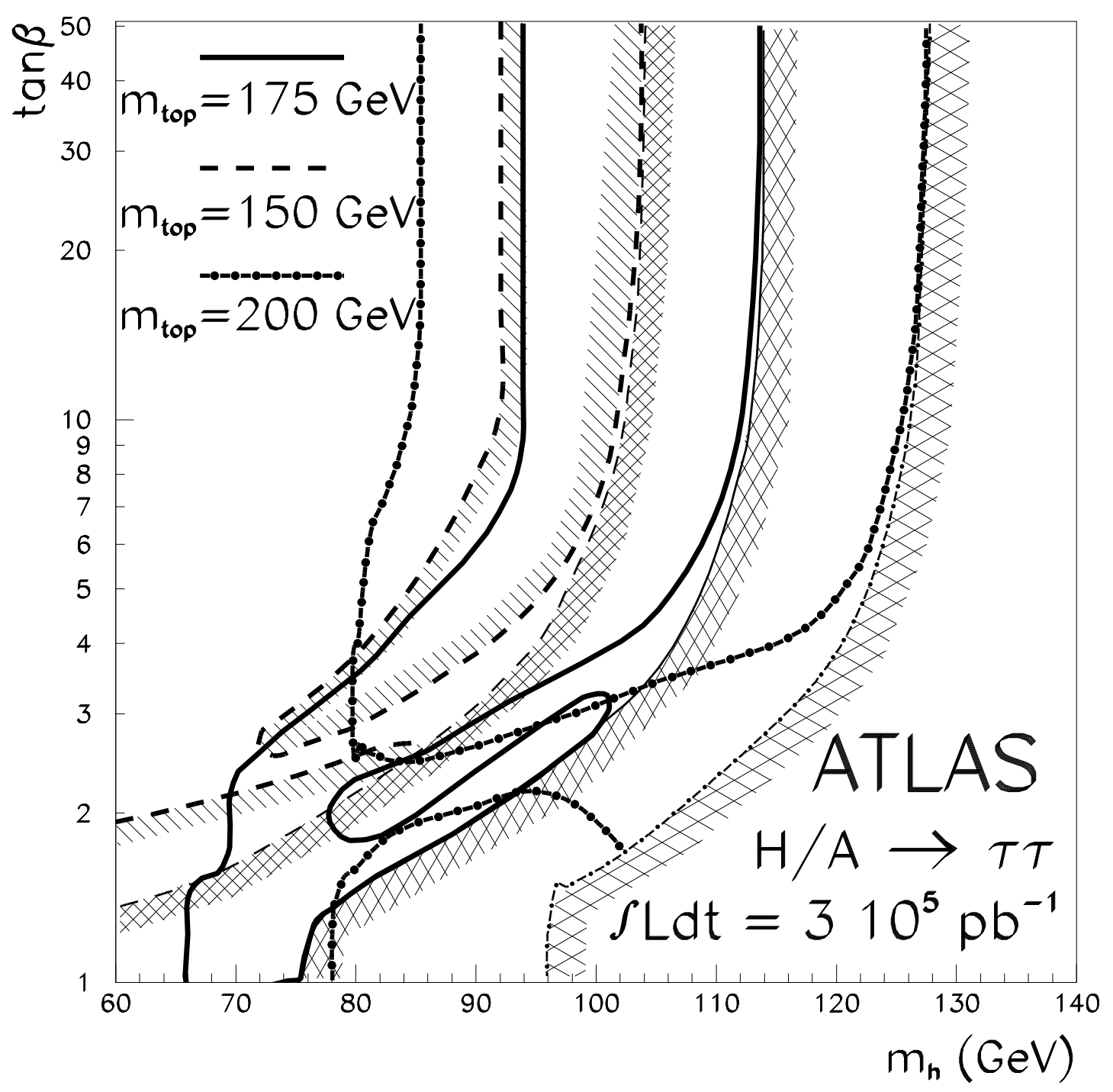

Figure 55: For $m_{\mathrm{t}}=150,175$ and $200 \mathrm{GeV}$ and an integrated luminosity of $3 \cdot 10^{5} \mathrm{pb}^{-1}, 5 \sigma$-discovery contour curves for the combined $\mathrm{H} / \mathrm{A} \rightarrow \tau \tau$ channel in the $\left(m_{\mathrm{h}}, \tan \beta\right)$ plane. The cross-hatched areas are theoretically excluded. 


\subsection{The $\mathrm{H} / \mathrm{A} \rightarrow \mu \mu$ channel}

As for $\mathrm{H} / \mathrm{A} \rightarrow \tau \tau$, this channel cannot be observed in the SM case because of the limited expected rate and of the overwhelming backgrounds, but it can be observed in the MSSM case, due to the large enhancement of $\mathrm{H} / \mathrm{A} \rightarrow \mu \mu$ rates through $b \bar{b} H$ and $b \bar{b} A$ production expected for large values of $\tan \beta$. The rates for this channel are governed by the same couplings as for the $\tau \tau$ channel, but the branching ratio scales as $\left(m_{\mu} / m_{\tau}\right)^{2}$. This huge reduction in signal rate with respect to the $\tau \tau$ channel is however compensated to some extent by the much better experimental resolution achievable in the $\mu \mu$ mode.

This channel had not been studied at the time of the ATLAS TP, but is however presented in the CMS TP [22]. A particle-level simulation has been performed to study it and the detailed results are reported in Appendix B. Table 35 gives a summary of these results in the usual form of expected signal and background rates and expected significances at low and high luminosity. A reconstruction efficiency of $90 \%$ per muon and an efficiency of $90 \%$ for the dimuon mass reconstruction in the chosen mass bin, $m_{\mathrm{H}} \pm 1.64 \sqrt{\left(\Gamma_{\mathrm{H}}^{\text {tot }} / 2.36\right)^{2}+{\sigma_{m}}^{2}}$, are assumed. The mass resolution $\sigma_{m}$ is approximately $\sigma_{m} / m_{\mathrm{H}}=2 \%$ [18]. The background is dominantly from $\mathrm{Z} / \gamma^{*} \rightarrow \mu \mu$ production, with an additional 20 to $30 \%$ contribution from $t \bar{t}$ production. The observability of the signal therefore depends only very weakly on $m_{t}$.

The expected $5 \sigma$-discovery contour curves for the combined $\mathrm{H} / \mathrm{A} \rightarrow \mu \mu$ signal are shown in Figs. 56 to 58.

Table 35: Observability of the $\mathrm{H} / \mathrm{A} \rightarrow \mu \mu$ channel at low and high luminosities. The $\sigma \times B R$ values and the expected numbers of signal and background events are given for $\mathrm{A} \rightarrow \mu \mu$ decays and for $\tan \beta=15$ (see Appendix B).

\begin{tabular}{|c|c||c|c|c||c|c|c|}
\hline \multicolumn{2}{|c||}{} & \multicolumn{3}{c||}{ Low luminosity } & \multicolumn{3}{c|}{ High luminosity } \\
\cline { 3 - 8 } \multicolumn{2}{|c||}{} & \multicolumn{3}{c|}{$3 \cdot 10^{4} \mathrm{pb}^{-1} \mathrm{pb}^{-1}$} \\
\hline$m_{\mathrm{A}}$ & $\begin{array}{c}\sigma \times B R \\
(\mathrm{GeV})\end{array}$ & Signal & Background & Signif. & Signal & Background & Signif. \\
\hline \hline 120.0 & 36.6 & 492 & 81700 & 1.7 & 1640 & 272400 & 3.1 \\
150.0 & 14.2 & 202 & 25800 & 1.3 & 672 & 86000 & 2.3 \\
200.0 & 5.32 & 78 & 10800 & 0.8 & 260 & 35900 & 1.4 \\
300.0 & 1.12 & 17 & 3200 & 0.3 & 58 & 10500 & 0.6 \\
400.0 & 0.36 & 6 & 1200 & 0.2 & 20 & 4100 & 0.3 \\
500.0 & 0.13 & 2 & 600 & 0.1 & 7 & 1900 & 0.2 \\
\hline
\end{tabular}




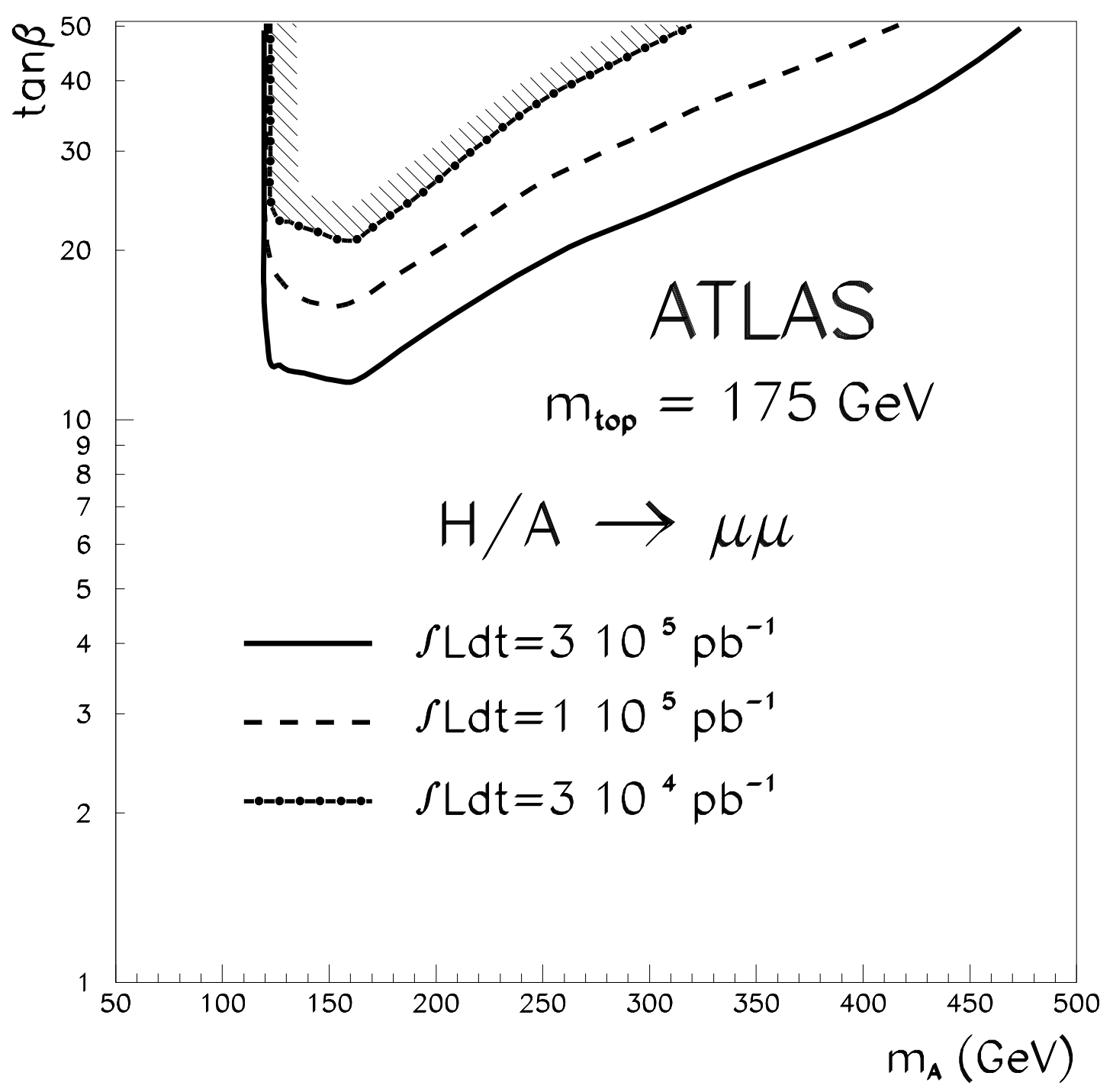

Figure 56: For $m_{\mathrm{t}}=175 \mathrm{GeV}$ and integrated luminosities of $3 \cdot 10^{4} \mathrm{pb}^{-1}$, $10^{5} \mathrm{pb}^{-1}$ and $3 \cdot 10^{5} \mathrm{pb}^{-1}, 5 \sigma$-discovery contour curves for the combined $\mathrm{H} / \mathrm{A} \rightarrow \mu \mu$ channel in the $\left(m_{\mathrm{A}}, \tan \beta\right)$ plane. 


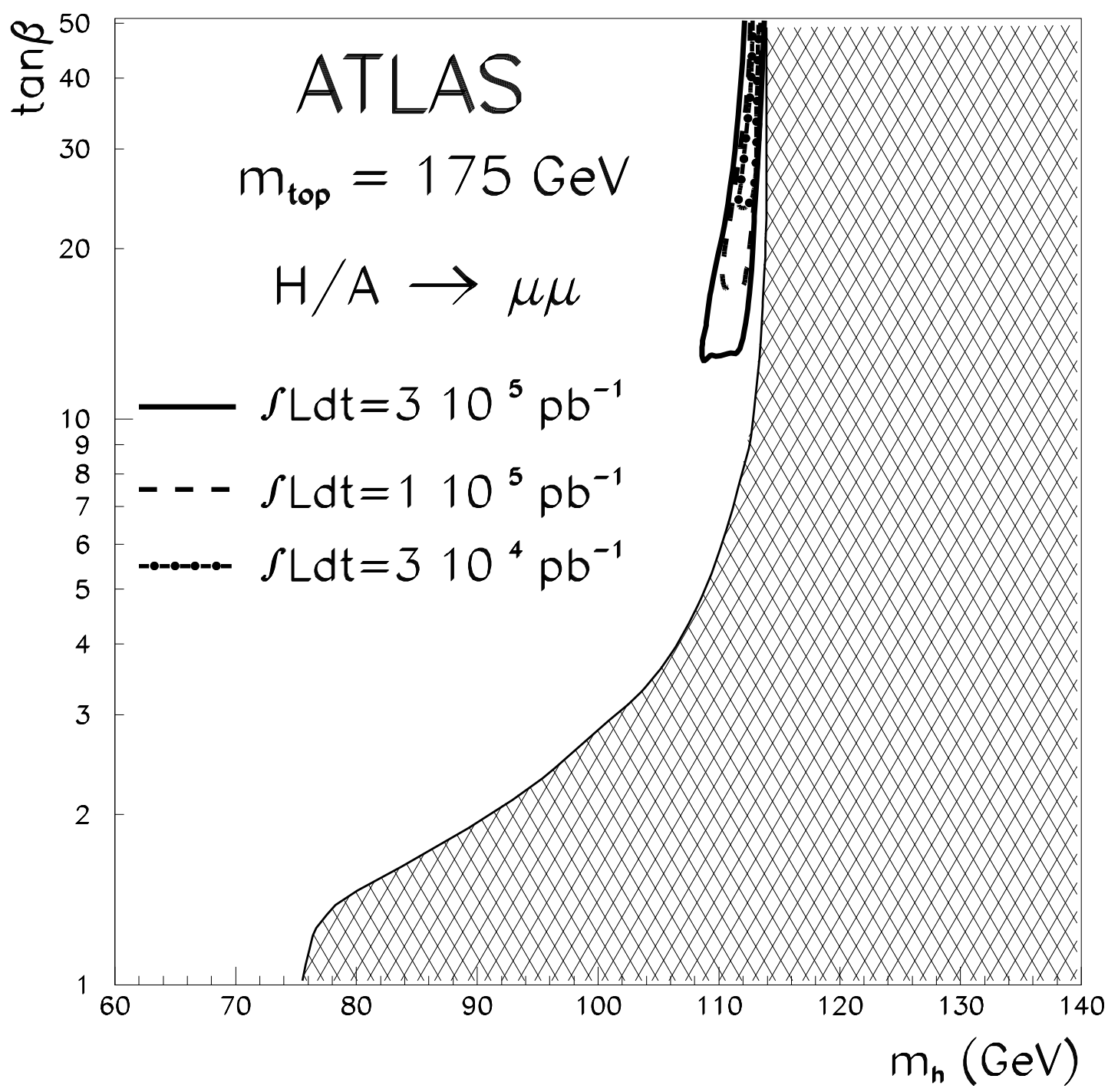

Figure 57: For $m_{\mathrm{t}}=175 \mathrm{GeV}$ and integrated luminosities of $3 \cdot 10^{4} \mathrm{pb}^{-1}$, $10^{5} \mathrm{pb}^{-1}$ and $3 \cdot 10^{5} \mathrm{pb}^{-1}, 5 \sigma$-discovery contour curves for the combined $\mathrm{H} / \mathrm{A} \rightarrow \mu \mu$ channel in the $\left(m_{\mathrm{h}}, \tan \beta\right)$ plane. The cross-hatched area is theoretically excluded. 


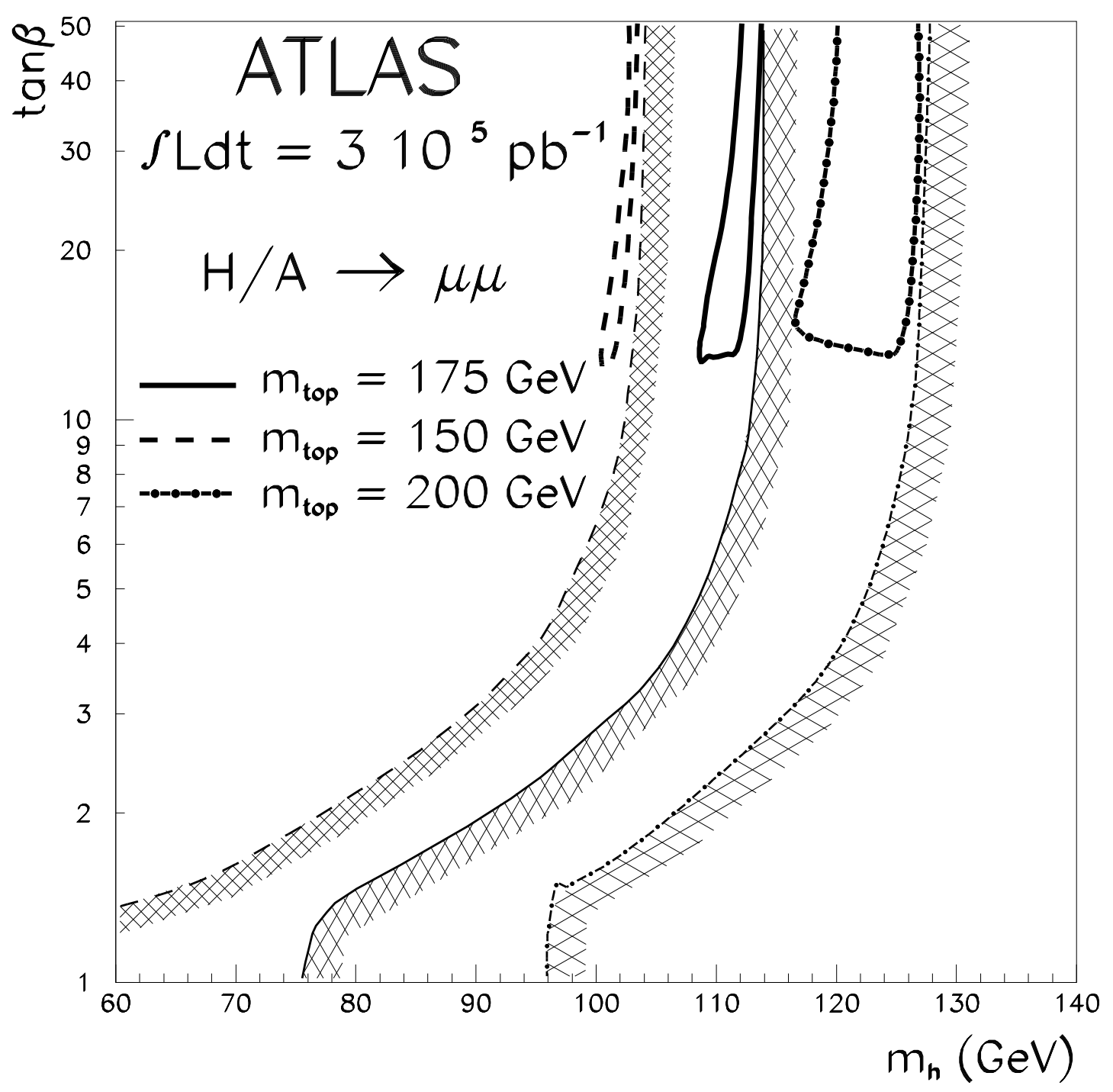

Figure 58: For $m_{\mathrm{t}}=150,175$ and $200 \mathrm{GeV}$ and an integrated luminosity of $3 \cdot 10^{5} \mathrm{pb}^{-1}, 5 \sigma$-discovery contour curves for the combined $\mathrm{H} / \mathrm{A} \rightarrow \mu \mu$ channel in the $\left(m_{\mathrm{h}}, \tan \beta\right)$ plane. The cross-hatched areas are theoretically excluded. 


\subsection{The $\mathrm{H} \rightarrow$ hh channel}

The observation of this channel would be particularly interesting, since it would correspond to the simultaneous discovery of two Higgs bosons. Possible final states of interest are:

1. $\mathrm{H} \rightarrow \mathrm{hh} \rightarrow \mathrm{b} \overline{\mathrm{b}} \mathrm{b} \overline{\mathrm{b}}$. This would provide the largest signal rate, but would require a 4 -jet trigger with as low a $\mathrm{p}_{\mathrm{T}}$-threshold as possible. Such a trigger is at present under study [23], as is the possibility of triggering on one low- $\mathrm{p}_{\mathrm{T}}$ muon from B-decay plus three additional jets. The b-tagging performance needed to control the overwhelming backgrounds from 4-jet events should be evaluated, as well as the irreducible $\mathrm{b} \overline{\mathrm{b}} \mathrm{b} \overline{\mathrm{b}}$ continuum background [24];

2. $\mathrm{H} \rightarrow \mathrm{hh} \rightarrow \mathrm{b} \overline{\mathrm{b}} \tau \tau$. At least one lepton from $\tau$-decay would be required to trigger the experiment, and the mass reconstruction of the $\tau \tau$-pair would follow that described for $\mathrm{H} / \mathrm{A} \rightarrow \tau \tau$ decays. The dominant backgrounds would be from $\mathrm{t} \overline{\mathrm{t}}$ and $\mathrm{W}+$ jet production;

3. $\mathrm{H} \rightarrow \mathrm{hh} \rightarrow \mathrm{b} \overline{\mathrm{b}} \gamma \gamma$. This channel was the only one studied for this note, because it can be easily triggered upon and it offers good kinematic constraints for the reconstruction of $m_{\mathrm{H}}$. For this reason, the study was extended to values of $m_{\mathrm{h}}$ as low as $60 \mathrm{GeV}$. Details can be found in Appendix C.

The signal was extracted by requiring two isolated photons, with $|\eta|<2.5$ and $\mathrm{p}_{\mathrm{T}}>20 \mathrm{GeV}$, and two additional jets with $|\eta|<2.5$ and $\mathrm{p}_{\mathrm{T}}>15 \mathrm{GeV}$ (resp. $\mathrm{p}_{\mathrm{T}}>30 \mathrm{GeV}$ ) at low (resp. high) luminosity. At least one of these jets was required to be tagged as a b-jet with an assumed efficiency $\epsilon_{b}=60 \%$ (resp. 50\%) at low (resp. high) luminosity. Events were accepted if the diphoton mass was within $\pm 2 \mathrm{GeV}$ of $m_{\mathrm{h}}$, and if the dijet mass was within $\pm 20 \mathrm{GeV}$ of $m_{\mathrm{h}}-20 \mathrm{GeV}$ (no correction to the reconstructed dijet mass was applied in this study). Finally, after rescaling the photon and jet 4-momenta appropriately by applying a constraint on $m_{\mathrm{h}}$, the $\gamma \gamma \mathrm{jj}$ invariant mass was required to be within $\pm 10 \mathrm{GeV}$ of $m_{\mathrm{H}}$.

Several background sources were considered: irreducible $\mathrm{b} \overline{\mathrm{b}} \gamma \gamma$ and reducible $\operatorname{bj} \gamma \gamma, \mathrm{c} \overline{\mathrm{c}} \gamma \gamma, \mathrm{cj} \gamma \gamma$ and $\mathrm{jj} \gamma \gamma$, which were all estimated using PYTHIA. Large uncertainties apply to these background estimates, due to the poor knowledge of the total $\mathrm{b} \overline{\mathrm{b}}, \mathrm{c} \overline{\mathrm{c}}$ and $\mathrm{jj}$ cross-sections, and to the procedure used to simulate photon bremsstrahlung in these processes.

The expected signal rates are very low, even when requiring only one of the two jets in the final state to be tagged as a b-jet. The $\mathrm{H} \rightarrow$ hh channel can be observed only for low values of $\tan \beta$ and for $200<m_{\mathrm{H}}<400 \mathrm{GeV}$. A few examples of the expected signal and background rates after selection cuts and of the significances at low and high luminosity are given in Table 36. For a given value of $m_{\mathrm{H}}$, the corresponding value of $m_{\mathrm{h}}$ increases as $\tan \beta$ increases, and the background rate therefore varies. The sensitivity to the signal for a 
given value of $m_{\mathrm{H}}$ was estimated for two different values of $m_{\mathrm{h}}$, and a simple linear interpolation or extrapolation was performed to obtain the $5 \sigma$-discovery contour curves in the $\left(m_{\mathrm{A}}, \tan \beta\right)$ and $\left(m_{\mathrm{h}}, \tan \beta\right)$ planes shown in Figs. 59 to 62 . The dependence on $m_{\mathrm{t}}$, which can be observed in Fig. 60, arises from changes in the $H \rightarrow$ hh branching ratio and in the value of $m_{\mathrm{h}}$ as a function of $m_{\mathrm{H}}$ and of $\tan \beta$. This channel can only be observed for low values of $\tan \beta, \tan \beta<4$, and for $2 m_{\mathrm{h}}<m_{\mathrm{H}}<2 m_{\mathrm{t}}$.

Table 36: Observability of the $\mathrm{H} \rightarrow \mathrm{hh} \rightarrow \mathrm{b} \overline{\mathrm{b}} \gamma \gamma$ channel at low and high luminosities. The $\sigma \times B R$ values and the expected numbers of signal and background events are given for $m_{\mathrm{h}} \sim 72 \mathrm{GeV}(\tan \beta=1)$ and for $m_{\mathrm{h}} \sim 97 \mathrm{GeV}$ $(\tan \beta=3)$. For more details, see Appendix $C$ and Tables 46 and $4 \%$

\begin{tabular}{|c|c|c||c|c|c||c|c|c|}
\hline \multicolumn{2}{|c||}{} & \multicolumn{3}{c||}{ Low luminosity } & \multicolumn{3}{c|}{ High luminosity } \\
\cline { 4 - 9 } & \multicolumn{3}{|c|}{$3 \cdot 10^{4} \mathrm{pb}^{-1}$} & \multicolumn{3}{c|}{$10^{5} \mathrm{pb}^{-1}$} \\
\hline $\begin{array}{c}m_{\mathrm{H}} \\
(\mathrm{GeV})\end{array}$ & $\begin{array}{c}\sigma \times B R \\
(\mathrm{fb})\end{array}$ & $\begin{array}{c}m_{\mathrm{h}} \\
(\mathrm{GeV})\end{array}$ & Signal & Background & Signif. & Signal & Background & Signif. \\
\hline \hline 231.0 & 6.0 & 71.2 & 13.7 & 1.3 & 6.4 & 15.7 & 1.4 & 7.3 \\
210.0 & 2.0 & 95.5 & 4.9 & 3.2 & 2.1 & 4.6 & 2.1 & 2.1 \\
\hline 275.0 & 5.0 & 72.4 & 11.9 & 0.2 & $>8.3$ & 21.1 & 0.5 & $>8.3$ \\
258.0 & 1.6 & 97.4 & 3.7 & 1.1 & 2.0 & 4.3 & 0.8 & 3.0 \\
\hline 321.0 & 4.6 & 73.0 & 11.9 & $\mathbf{0 . 2}$ & $>8.3$ & 28.4 & 0.4 & $>8.3$ \\
306.0 & 1.2 & 98.3 & 3.5 & 0.6 & 2.7 & 5.8 & 1.0 & 3.2 \\
\hline 368.0 & 0.9 & 73.4 & 3.4 & $\mathbf{0 . 1}$ & 3.6 & 7.6 & 0.3 & 5.4 \\
355.0 & 1.0 & 98.8 & 3.3 & $\mathbf{0 . 4}$ & 2.4 & 6.8 & 0.7 & 4.2 \\
\hline
\end{tabular}




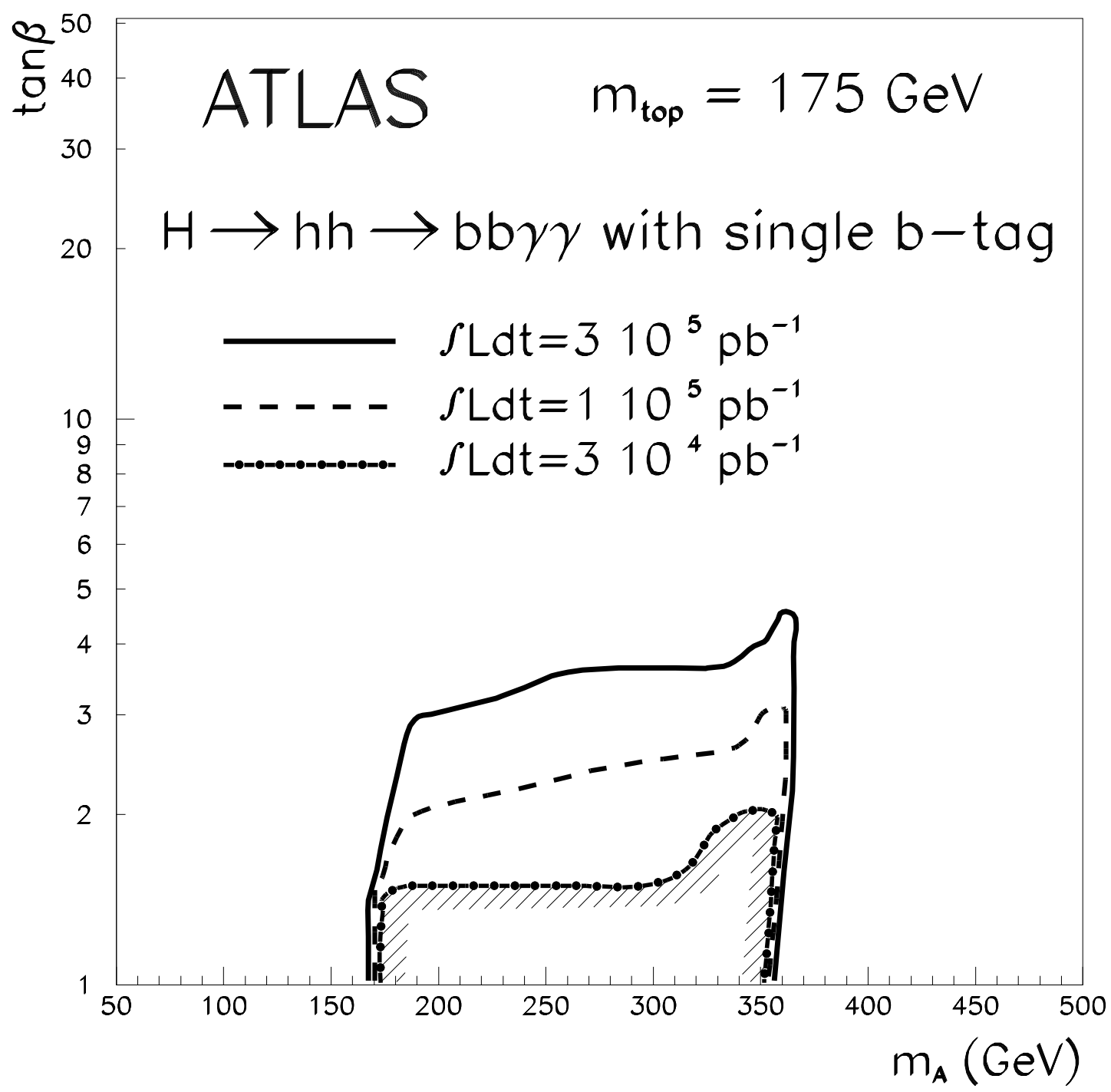

Figure 59: For $m_{\mathrm{t}}=175 \mathrm{GeV}$ and integrated luminosities of $3 \cdot 10^{4} \mathrm{pb}^{-1}, 10^{5} \mathrm{pb}^{-1}$ and $3 \cdot 10^{5} \mathrm{pb}^{-1}, 5 \sigma$-discovery contour curves for the $\mathrm{H} \rightarrow \mathrm{hh} \rightarrow \mathrm{b} \overline{\mathrm{b}} \gamma \gamma$ channel in the $\left(m_{\mathrm{A}}, \tan \beta\right)$ plane. 


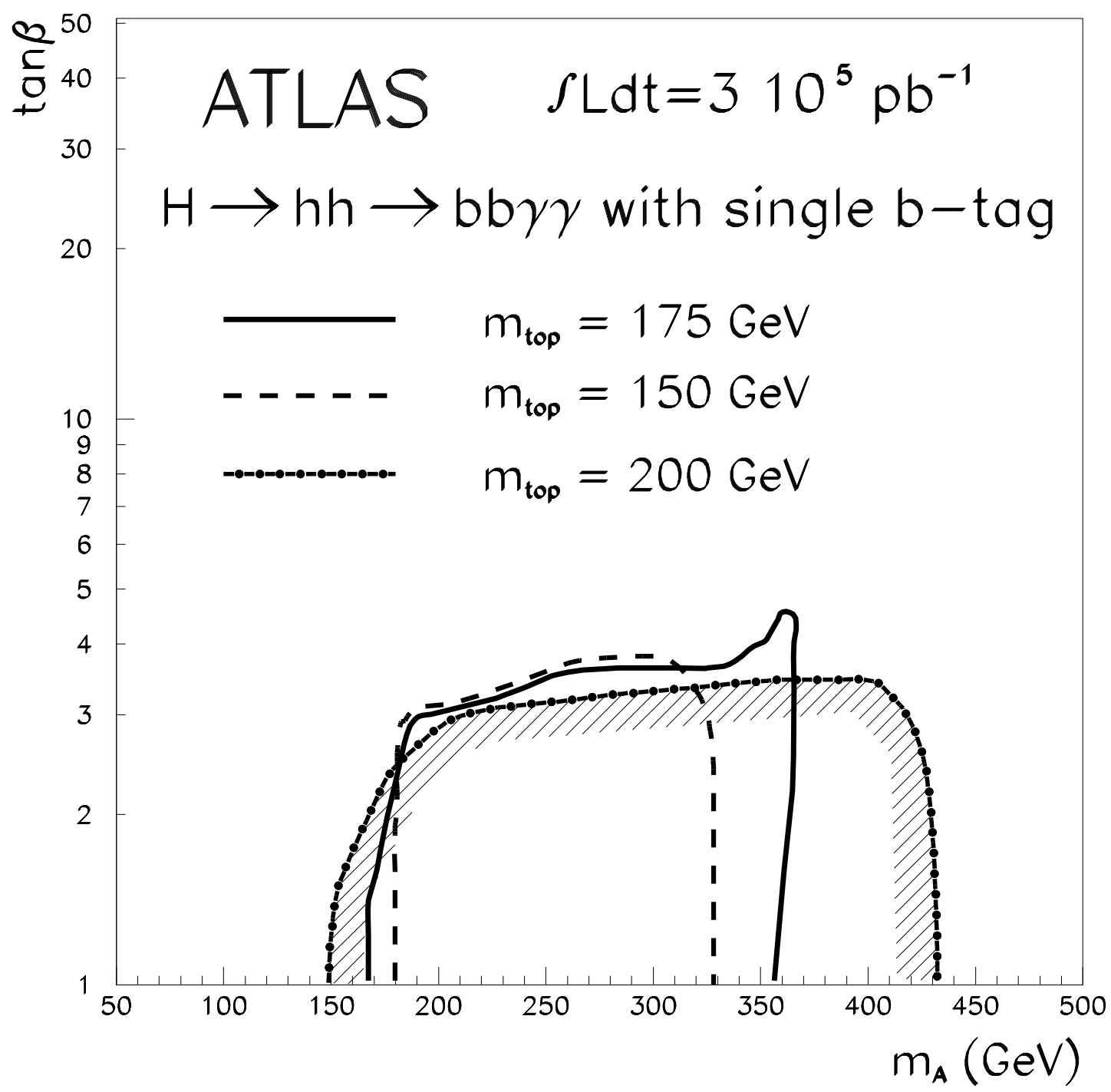

Figure 60: For $m_{\mathrm{t}}=150,175$ and $200 \mathrm{GeV}$ and an integrated luminosity of $3 \cdot 10^{5} \mathrm{pb}^{-1}, 5 \sigma$-discovery contour curves for the $\mathrm{H} \rightarrow \mathrm{hh} \rightarrow \mathrm{b} \overline{\mathrm{b}} \gamma \gamma$ channel in the $\left(m_{\mathrm{A}}, \tan \beta\right)$ plane. 


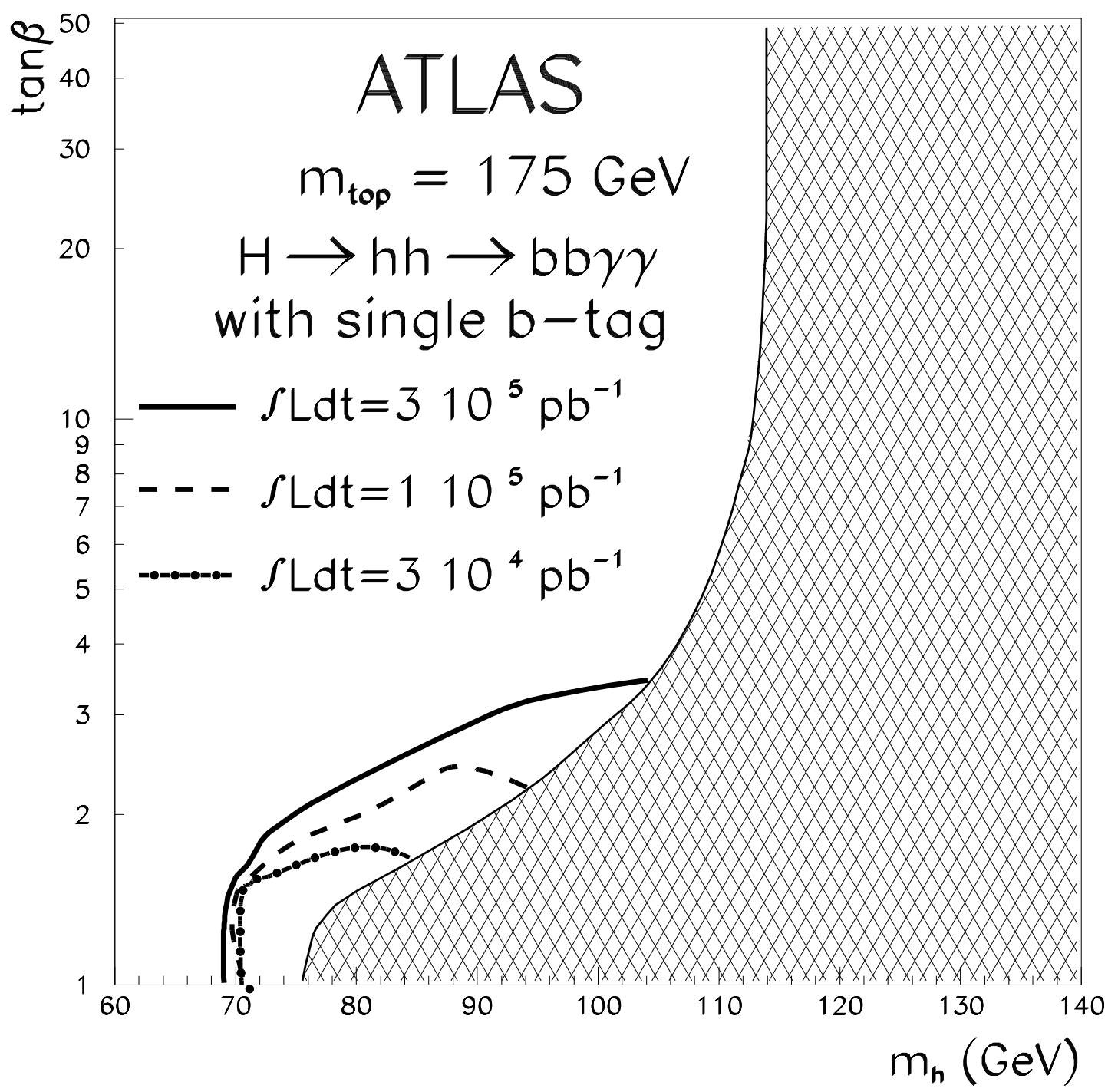

Figure

61:

For $m_{\mathrm{t}}=175 \mathrm{GeV}$ and integrated luminosities of $3 \cdot 10^{4} \mathrm{pb}^{-1}, 10^{5} \mathrm{pb}^{-1}$ and $3 \cdot 10^{5} \mathrm{pb}^{-1}, 5 \sigma$-discovery contour curves for the $\mathrm{H} \rightarrow \mathrm{hh} \rightarrow \mathrm{b} \overline{\mathrm{b}} \gamma \gamma$ channel in the $\left(m_{\mathrm{h}}, \tan \beta\right)$ plane. The cross-hatched area is theoretically excluded. 


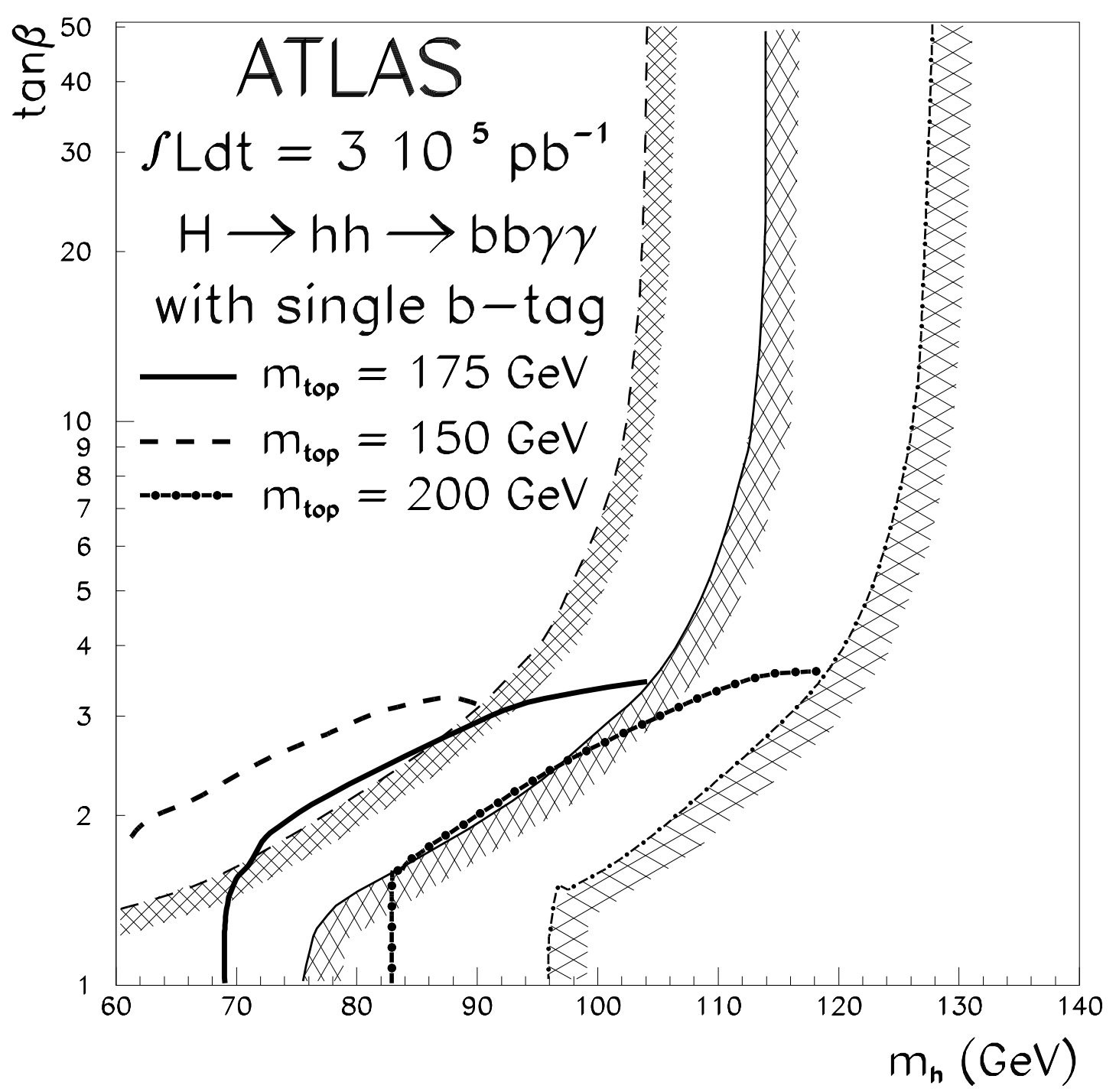

Figure 62: For $m_{\mathrm{t}}=150,175$ and $200 \mathrm{GeV}$ and an integrated luminosity of $3 \cdot 10^{5} \mathrm{pb}^{-1}, 5 \sigma$-discovery contour curves for the $\mathrm{H} \rightarrow \mathrm{hh} \rightarrow \mathrm{b} \overline{\mathrm{b}} \gamma \gamma$ channel in the $\left(m_{\mathrm{h}}, \tan \beta\right)$ plane. The cross-hatched areas are theoretically excluded. 


\subsection{The $\mathrm{H} / \mathrm{A} \rightarrow \mathrm{t} \overline{\mathrm{t}}$ channel}

Because of the strong couplings of the SM Higgs boson to gauge boson pairs, the $\mathrm{H} \rightarrow \mathrm{t} \overline{\mathrm{t}}$ branching ratio is too small for this channel to be observable in the $\mathrm{SM}$ case. In the MSSM case, however, the $\mathrm{H} \rightarrow \mathrm{t} \overline{\mathrm{t}}$ and $\mathrm{A} \rightarrow \mathrm{t} \overline{\mathrm{t}}$ branching ratios are close to $100 \%$ for $m_{\mathrm{H}}, m_{\mathrm{A}}>2 m_{\mathrm{t}}$ and for $\tan \beta \sim 1$. The $\mathrm{H} \rightarrow \mathrm{t} \overline{\mathrm{t}}$ and $\mathrm{A} \rightarrow \mathrm{t} \overline{\mathrm{t}}$ decays cannot be distinguished experimentally from each other, since the $\mathrm{H}$ - and A-bosons are almost degenerate in mass in the relevant region of parameter space. As discussed in the literature [25], a signal from $\mathrm{H} / \mathrm{A} \rightarrow \mathrm{t} \overline{\mathrm{t}}$ decays would only appear as a peak in the $\mathrm{t} \overline{\mathrm{t}}$ invariant mass

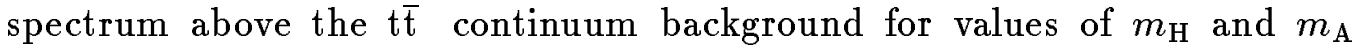
smaller than $\sim 500 \mathrm{GeV}$, due to negative interference effects between the signal and background amplitudes.

The results of a detailed particle-level simulation of this channel are presented in Appendix D and summarised here. The signal was extracted by searching for $\mathrm{WWb} \bar{b}$ final states, with one $\mathrm{W} \rightarrow \ell \nu$ and one $\mathrm{W} \rightarrow \mathrm{jj}$ decay. The lepton was required to have $\mathrm{p}_{\mathrm{T}}>20 \mathrm{GeV}$ and all the jets, i.e. those from $W$-decay and the two b-jets, were required to have $\mathrm{p}_{\mathrm{T}}>40 \mathrm{GeV}$. It was assumed that the experiment could trigger on such topologies and efficiently reconstruct them at low and high luminosities. Both b-jets were required to be tagged, with an assumed efficiency $\epsilon_{b}=60 \%$ (resp. 50\%) at low (resp. high) luminosity.

Both top-quark decays were fully reconstructed and a constraint on $m_{\mathrm{t}}$ was used to improve the experimental resolution on the $t \bar{t}$ invariant mass. The expected mass resolution for $\mathrm{H} / \mathrm{A} \rightarrow \mathrm{t} \overline{\mathrm{t}}$ decays increases from 40 to $80 \mathrm{GeV}$ as $m_{\mathrm{H}}$ and $m_{\mathrm{A}}$ increase from 400 to $500 \mathrm{GeV}$.

The background from continuum t $\bar{t}$ production is much larger than the $\mathrm{W}+$ jet background after these selection cuts, and is unfortunately also much larger than the signal, as shown in Table 37. The signal-to-background ratio varies between $1.5 \%$ and $7 \%$ over the range of Higgs boson and top-quark masses considered.

The mass resolutions quoted above imply that a typical mass window allowing to observe most of the signal would be between 150 and $300 \mathrm{GeV}$. With such wide mass windows, the signal can only be observed above the continuum background as an excess of events. This excess would be very significant statistically, as shown in Table 37 , but this significance would only

be meaningful if the theoretical uncertainties on the continuum background shape were lower than a percent or so. 
Although the theoretical uncertainties on continuum t $\bar{t}$ production are much larger than this today, it is hoped that they could be reduced with time, and that the experimental data at the $\mathrm{LHC}$ would also contribute to a better understanding of heavy flavour continuum production. Bearing this optimistic scenario in mind, the numbers quoted in Table 37 have been used to extract the $5 \sigma$-discovery contour curves for $\mathrm{H} / \mathrm{A} \rightarrow \mathrm{t} \overline{\mathrm{t}}$ decays shown in Figs. 63 to 66 . These curves cover at best a limited region in parameter space, i.e. $m_{\mathrm{H}}, m_{\mathrm{A}}>2 m_{\mathrm{t}}$ and $\tan \beta<3$. For larger values of $\tan \beta$, the $\mathrm{H} / \mathrm{A} \rightarrow \mathrm{b} \overline{\mathrm{b}}$ branching ratios become dominant.

Table 37: Observability of the $\mathrm{H} / \mathrm{A} \rightarrow \mathrm{t} \overline{\mathrm{t}}$ channel at low and high luminosities. The $\sigma \times B R$ values and the expected numbers of signal and background events are given for combined $\mathrm{H} / \mathrm{A} \rightarrow \mathrm{t} \overline{\mathrm{t}}$ decays and for $\tan \beta=1.5$ (see Appendix D).

\begin{tabular}{|c|c|c|c|c|c|c|c|}
\hline \multirow{2}{*}{$\begin{array}{c} \\
m_{\mathrm{H}}, m_{\mathrm{A}} \\
(\mathrm{GeV}) \\
\end{array}$} & \multirow[b]{2}{*}{$\begin{array}{c}\sigma \times B R \\
(\mathrm{pb})\end{array}$} & \multicolumn{3}{|c|}{$\begin{array}{c}\text { Low luminosity } \\
3 \cdot 10^{4} \mathrm{pb}^{-1}\end{array}$} & \multicolumn{3}{|c|}{$\frac{\text { High luminosity }}{10^{5} \mathrm{pb}^{-1}}$} \\
\hline & & Signal & Background & Signif. & Signal & Background & Signif. \\
\hline & & \multicolumn{6}{|c|}{$m_{\mathrm{t}}=150 \mathrm{GeV}$} \\
\hline 330.0 & 10.5 & 2750 & 95600 & 8.9 & 6370 & 220500 & 13.6 \\
\hline 370.0 & 8.0 & 2650 & 95300 & 8.6 & 6130 & 220500 & 12.9 \\
\hline 400.0 & 6.2 & 2460 & 104800 & 7.6 & 5700 & 242500 & 11.4 \\
\hline 450.0 & 4.0 & 2220 & 133400 & 6.0 & 5130 & 308700 & 9.20 \\
\hline \multirow[t]{2}{*}{500.0} & 2.9 & 2120 & 161900 & 5.3 & 4900 & 374800 & 7.90 \\
\hline & & \multicolumn{6}{|c|}{$m_{\mathrm{t}}=175 \mathrm{GeV}$} \\
\hline 370.0 & 11.8 & 4360 & 68600 & 16.7 & 10100 & 158700 & 25.3 \\
\hline 400.0 & 8.40 & 4000 & 85700 & 13.7 & 9270 & 198500 & 20.9 \\
\hline 450.0 & 4.80 & 3270 & 107800 & 10.0 & 7570 & 249500 & 15.2 \\
\hline \multirow[t]{2}{*}{500.0} & 2.90 & 2450 & 127400 & 6.9 & 5670 & 294800 & 10.4 \\
\hline & & \multicolumn{6}{|c|}{$m_{\mathrm{t}}=200 \mathrm{GeV}$} \\
\hline 450.0 & 6.0 & 4260 & 63400 & 16.9 & 99870 & 146700 & 25.6 \\
\hline 500.0 & 3.8 & 3590 & 79700 & 12.7 & 8300 & 184500 & 19.3 \\
\hline
\end{tabular}




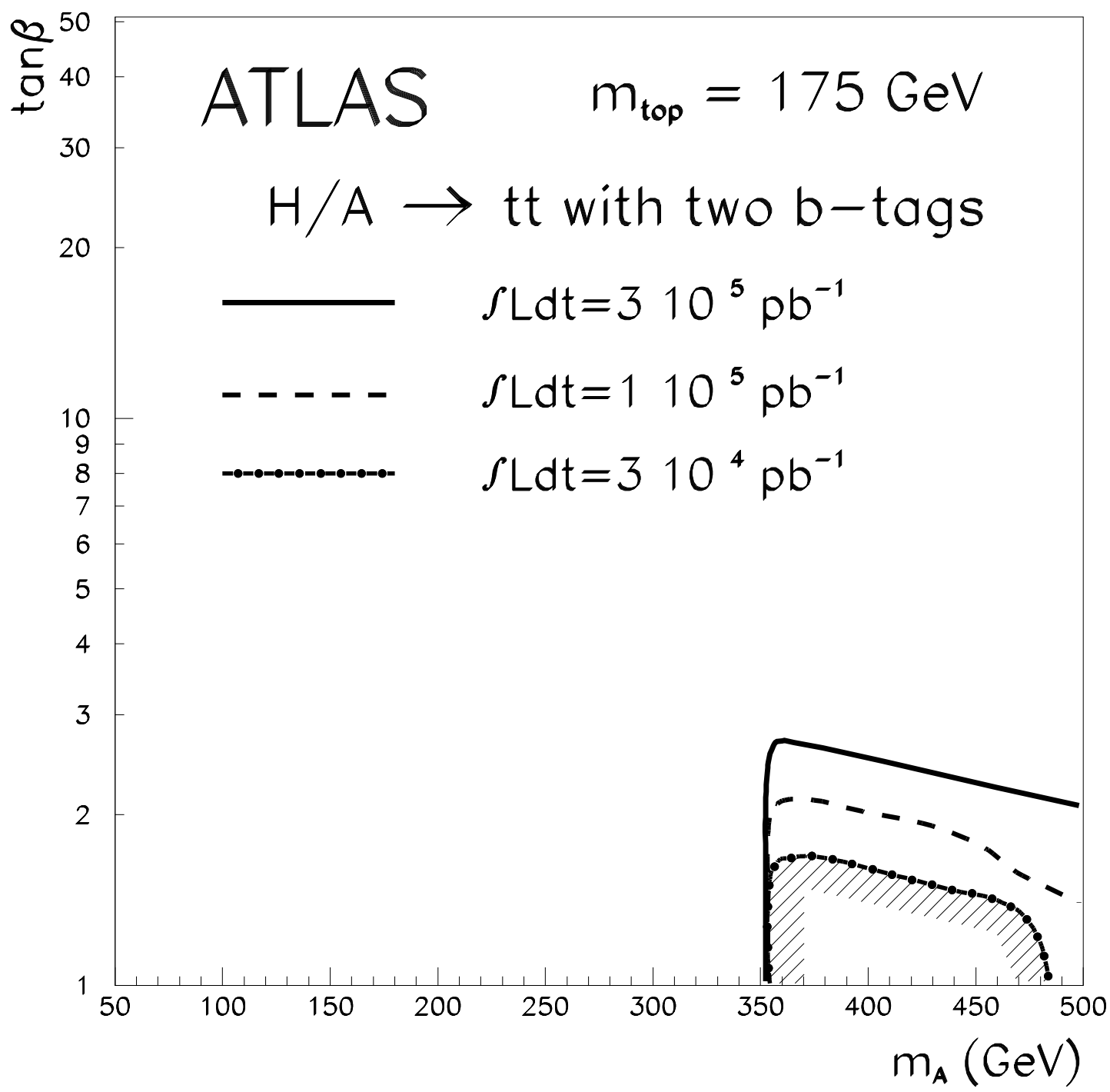

Figure 63: For $m_{\mathrm{t}}=175 \mathrm{GeV}$ and integrated luminosities of $3 \cdot 10^{4} \mathrm{pb}^{-1}$, $10^{5} \mathrm{pb}^{-1}$ and $3 \cdot 10^{5} \mathrm{pb}^{-1}, 5 \sigma$-discovery contour curves for the combined $\mathrm{H} / \mathrm{A} \rightarrow \mathrm{t} \overline{\mathrm{t}}$ channel in the $\left(m_{\mathrm{A}}, \tan \beta\right)$ plane. 


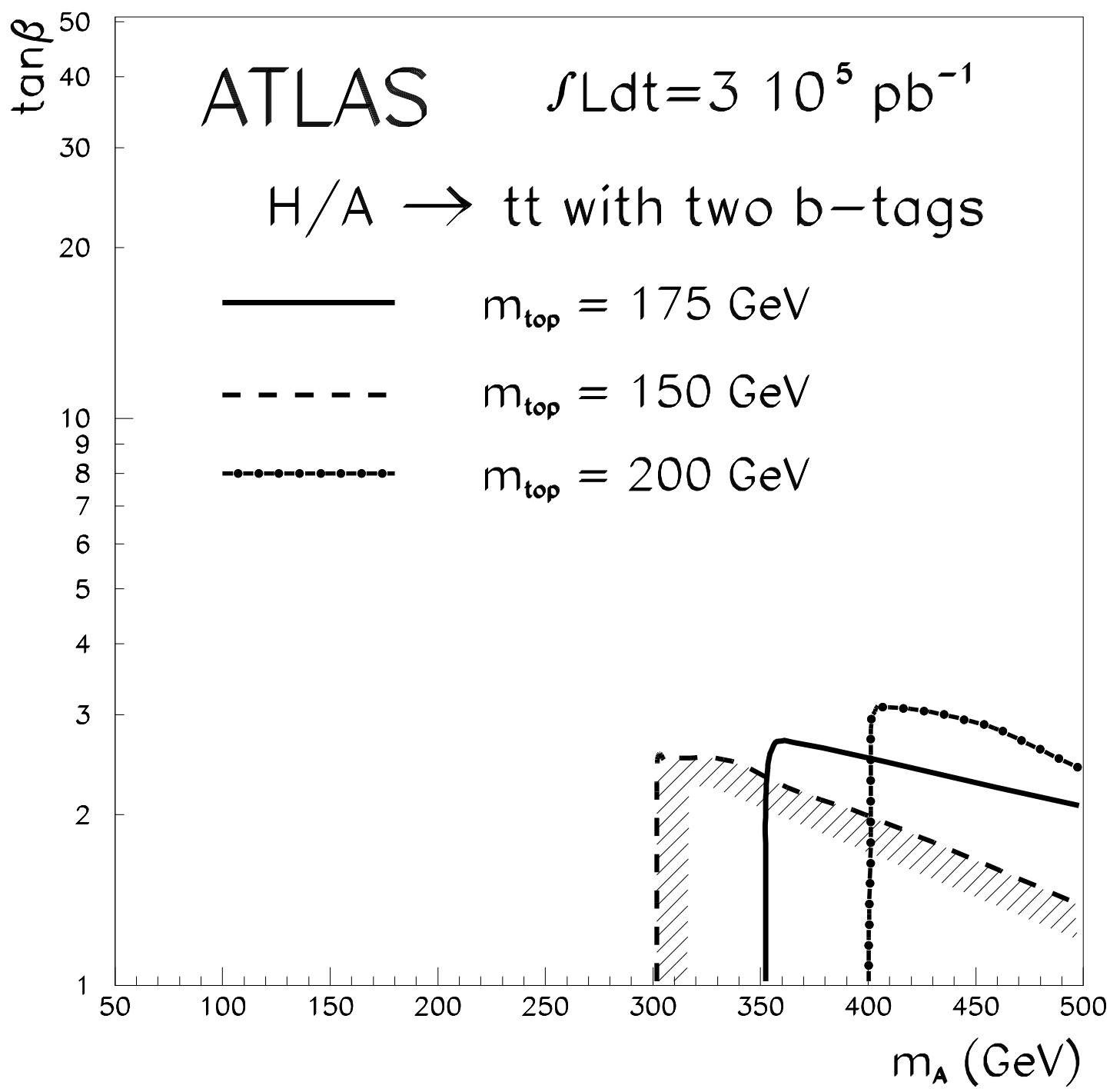

Figure 64: For $m_{\mathrm{t}}=150,175$ and $200 \mathrm{GeV}$ and an integrated luminosity of $3 \cdot 10^{5} \mathrm{pb}^{-1}, 5 \sigma$-discovery contour curves for the combined $\mathrm{H} / \mathrm{A} \rightarrow \mathrm{t} \overline{\mathrm{t}}$ channel in the $\left(m_{\mathrm{A}}, \tan \beta\right)$ plane. 


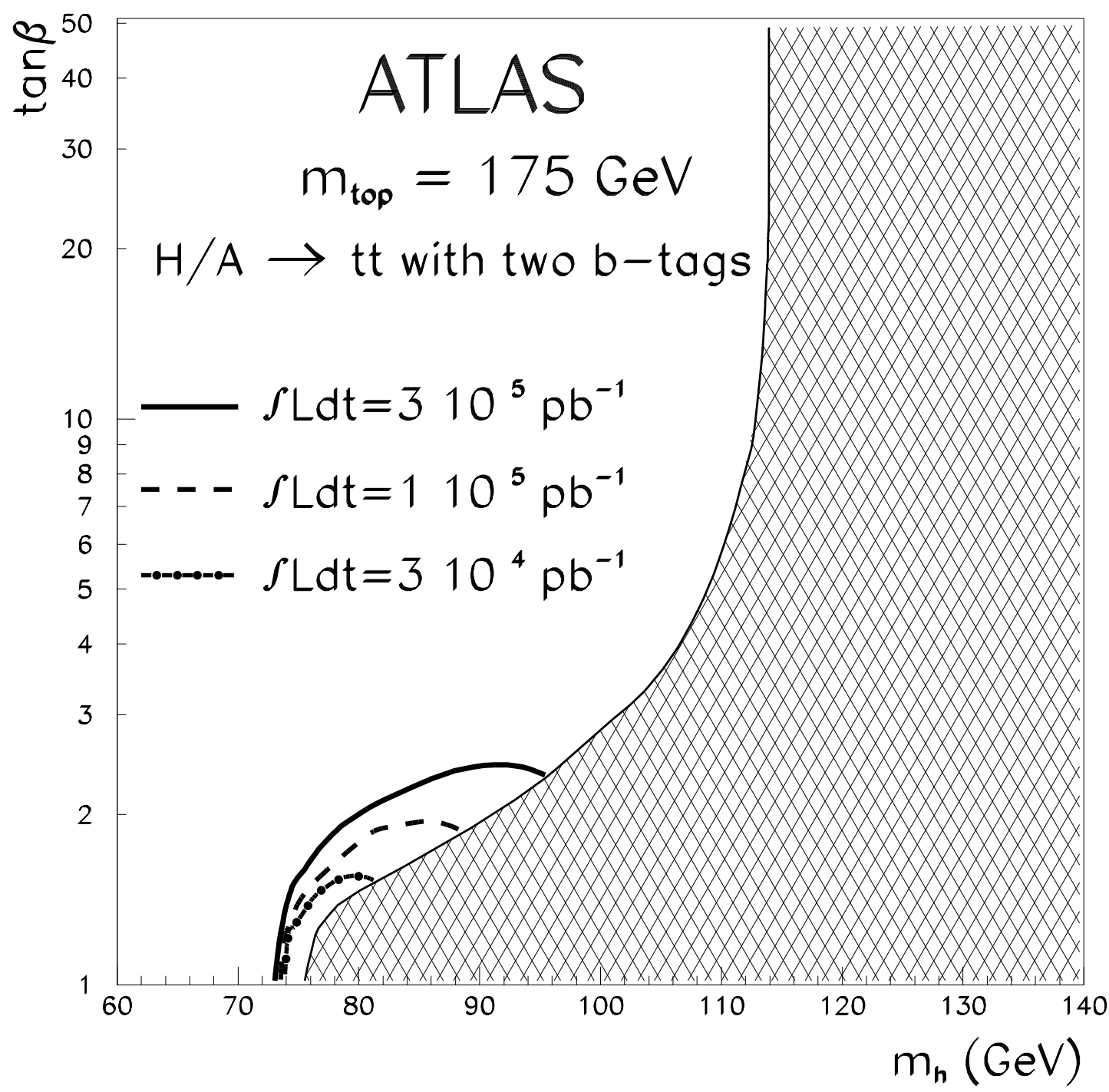

Figure 65: For $m_{\mathrm{t}}=175 \mathrm{GeV}$ and integrated luminosities of $3 \cdot 10^{4} \mathrm{pb}^{-1}$, $10^{5} \mathrm{pb}^{-1}$ and $3 \cdot 10^{5} \mathrm{pb}^{-1}, 5 \sigma$-discovery contour curves for the combined $\mathrm{H} / \mathrm{A} \rightarrow \mathrm{t} \overline{\mathrm{t}}$ channel in the $\left(m_{\mathrm{h}}, \tan \beta\right)$ plane. The cross-hatched area is theoretically excluded. 


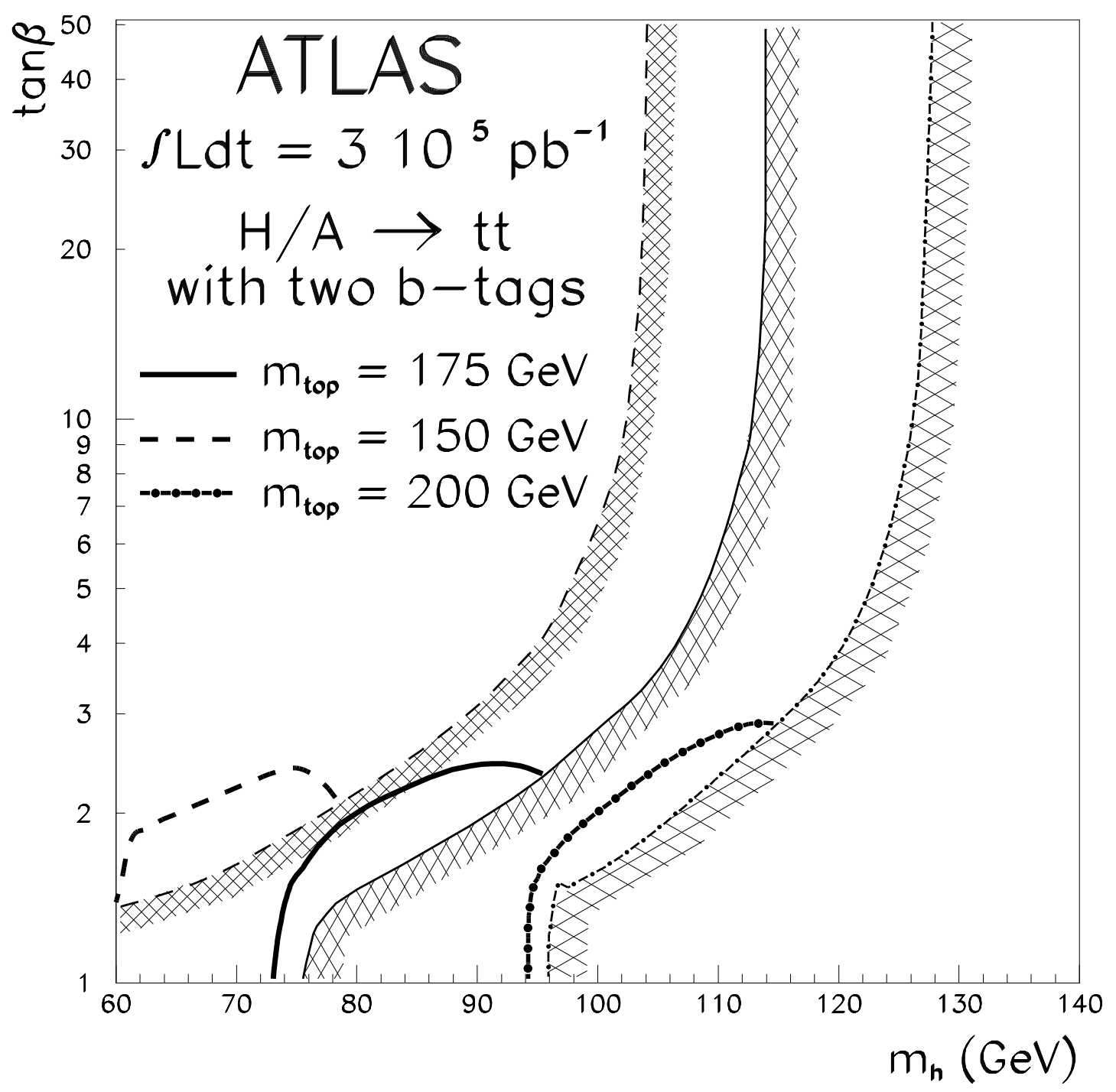

Figure 66: For $m_{\mathrm{t}}=150,175$ and $200 \mathrm{GeV}$ and an integrated luminosity of $3 \cdot 10^{5} \mathrm{pb}^{-1}, 5 \sigma$-discovery contour curves for the combined $\mathrm{H} / \mathrm{A} \rightarrow \mathrm{t} \overline{\mathrm{t}}$ channel in the $\left(m_{\mathrm{h}}, \tan \beta\right)$ plane. The cross-hatched areas are theoretically excluded. 


\subsection{The $\mathrm{A} \rightarrow \mathrm{Zh}$ channel}

The observation of this channel would be particularly interesting, since it would correspond to the simultaneous discovery of two Higgs bosons. It is the dominant $\mathrm{A}$-boson decay channel for low values of $\tan \beta$ and for $m_{\mathrm{Z}}+m_{\mathrm{h}}<m_{\mathrm{A}}<2 m_{\mathrm{t}}$. Possible final states of interest are:

1. $\mathrm{A} \rightarrow \mathrm{Zh} \rightarrow \mathrm{b} \overline{\mathrm{b}} \mathrm{b} \overline{\mathrm{b}}$, similarly to $\mathrm{H} \rightarrow \mathrm{hh}$ decays (see Section 5.6). This would provide the largest signal rate, but would require a 4 -jet trigger with as low a $\mathrm{p}_{\mathrm{T}}$-threshold as possible. Such a trigger is at present under study [23], as is the possibility of triggering on one low-p muon from B-decay plus three additional jets. The b-tagging performance needed to control the overwhelming backgrounds from 4-jet events should be evaluated, as well as the irreducible $b \bar{b} b \bar{b}$ continuum background [24];

2. $\mathrm{A} \rightarrow \mathrm{Zh} \rightarrow \ell \ell \mathrm{b} \overline{\mathrm{b}}$. This channel was the only one studied for this note, because it can be easily triggered upon and it offers the largest rates apart from the dominant channel discussed above. Details can be found in Appendix E;

3. A $\rightarrow \mathrm{Zh} \rightarrow \ell \ell \gamma \gamma$. This channel would provide better kinematic constraints in the final state than the preceding one, but the expected rates are too low for it to be observable at the LHC.

The signal was extracted by requiring two isolated leptons, with $|\eta|<2.5$ and $\mathrm{p}_{\mathrm{T}}>20 \mathrm{GeV}$, and two additional jets with $|\eta|<2.5$ and $\mathrm{p}_{\mathrm{T}}>15 \mathrm{GeV}$ (resp. $\mathrm{p}_{\mathrm{T}}>30 \mathrm{GeV}$ ) at low (resp. high) luminosity. Both jets were required to be tagged as b-jets with an assumed efficiency $\epsilon_{b}=60 \%$ (resp. $50 \%$ ) at low (resp. high) luminosity. Events were accepted if the dilepton mass was within $\pm 6 \mathrm{GeV}$ of $m_{\mathrm{Z}}$, and if the dijet mass was within $\pm 20 \mathrm{GeV}$ of $m_{\mathrm{h}}-$ $20 \mathrm{GeV}$ (no correction to the reconstructed dijet mass was applied in this study). Finally, after rescaling the lepton and jet 4-momenta appropriately by applying constraints on $m_{\mathrm{Z}}$ and $m_{\mathrm{h}}$, the $\ell \ell \mathrm{jj}$ invariant mass was required to be within $\pm 6 \mathrm{GeV}$ of $m_{\mathrm{A}}$. Several background sources were considered: irreducible $\mathrm{Zb} \overline{\mathrm{b}}$ and $\mathrm{ZZ}$, and reducible $\mathrm{ZW}, \mathrm{Zjj}$ and $\mathrm{t} \overline{\mathrm{t}}$. After the selection cuts, the $\mathrm{Zb} \overline{\mathrm{b}}$ and $\mathrm{t} \overline{\mathrm{t}}$ backgrounds are dominant (see Appendix $\mathrm{E}$ ).

The expected signal rates decrease very rapidly as $\tan \beta$ increases. The $A \rightarrow$ Zh channel can therefore only be observed for low values of $\tan \beta$ and for $200<m_{\mathrm{A}}<2 m_{\mathrm{t}}$. A few examples of the expected signal and background rates after selection cuts and of the significances at low and high luminosity are given in Table 38. For a given value of $m_{\mathrm{A}}$, the corresponding value of $m_{\mathrm{h}}$ increases as $\tan \beta$ increases, and the background rate therefore varies. The sensitivity to the signal for a given value of $m_{\mathrm{A}}$ was estimated for two different values of $m_{\mathrm{h}}$, and a simple linear interpolation or extrapolation was performed to obtain the $5 \sigma$-discovery contour curves in the $\left(m_{\mathrm{A}}, \tan \beta\right)$ and $\left(m_{\mathrm{h}}, \tan \beta\right)$ planes shown in Figs. 67 to 70. 
Table 38: Observability of the $\mathrm{A} \rightarrow \mathrm{Zh}$ channel with $\mathrm{Z} \rightarrow \ell \ell$ and $\mathrm{h} \rightarrow \mathrm{b} \overline{\mathrm{b}}$ decays at low and high luminosities. The $\sigma \times B R$ values and the expected numbers of signal and background events are given for $m_{\mathrm{h}} \sim 72 \mathrm{GeV}(\tan \beta=1)$ and for $m_{\mathrm{h}} \sim 97 \mathrm{GeV}(\tan \beta=3)$. For more details, see Appendix E and Tables 55 and 58.

\begin{tabular}{|c|c|c|c|c|c|c|c|c|}
\hline \multirow{2}{*}{$\begin{array}{c} \\
m_{\mathrm{A}} \\
(\mathrm{GeV})\end{array}$} & \multirow[b]{2}{*}{$\begin{array}{c}\sigma \times B R \\
(\mathrm{fb})\end{array}$} & \multirow[b]{2}{*}{$\begin{array}{c}m_{\mathrm{h}} \\
(\mathrm{GeV}) \\
\end{array}$} & \multicolumn{3}{|c|}{$\begin{array}{l}\text { Low luminosity } \\
3 \cdot 10^{4} \mathrm{pb}^{-1}\end{array}$} & \multicolumn{3}{|c|}{$\frac{\text { High luminosity }}{10^{5} \mathrm{pb}^{-1}}$} \\
\hline & & & Signal & Background & Signif. & Signal & Background & Signif. \\
\hline & & & \multicolumn{6}{|c|}{$m_{\mathrm{t}}=150 \mathrm{GeV}$} \\
\hline 200.0 & $\begin{array}{c}561 \\
9\end{array}$ & $\begin{array}{l}71 \\
96\end{array}$ & $\begin{array}{c}675 \\
15\end{array}$ & $\begin{array}{l}1170 \\
1690\end{array}$ & $\begin{array}{c}19.7 \\
0.4\end{array}$ & $\begin{array}{c}336 \\
12\end{array}$ & $\begin{array}{c}360 \\
1180\end{array}$ & $\begin{array}{c}17.8 \\
0.3\end{array}$ \\
\hline 250.0 & $\begin{array}{l}472 \\
21\end{array}$ & $\begin{array}{l}72 \\
97\end{array}$ & $\begin{array}{c}786 \\
39\end{array}$ & $\begin{array}{c}530 \\
1560\end{array}$ & $\begin{array}{c}34.3 \\
1.0\end{array}$ & $\begin{array}{c}840 \\
46\end{array}$ & $\begin{array}{c}320 \\
1170\end{array}$ & $\begin{array}{l}47.3 \\
1.4\end{array}$ \\
\hline 300.0 & $\begin{array}{c}341 \\
17\end{array}$ & $\begin{array}{l}73 \\
98\end{array}$ & $\begin{array}{c}642 \\
37\end{array}$ & $\begin{array}{l}200 \\
720\end{array}$ & $\begin{array}{c}45.4 \\
1.4\end{array}$ & $\begin{array}{c}1000 \\
57\end{array}$ & $\begin{array}{c}80 \\
630\end{array}$ & $\begin{array}{c}109.8 \\
2.3\end{array}$ \\
\hline & & & \multicolumn{6}{|c|}{$m_{\mathrm{t}}=175 \mathrm{GeV}$} \\
\hline 200.0 & $\begin{array}{c}561 \\
9\end{array}$ & $\begin{array}{l}71 \\
96\end{array}$ & $\begin{array}{c}675 \\
15\end{array}$ & $\begin{array}{c}970 \\
1140\end{array}$ & $\begin{array}{c}21.7 \\
0.5\end{array}$ & $\begin{array}{c}336 \\
12\end{array}$ & $\begin{array}{l}220 \\
610\end{array}$ & $\begin{array}{c}22.8 \\
0.5\end{array}$ \\
\hline 250.0 & $\begin{array}{l}472 \\
21\end{array}$ & $\begin{array}{l}72 \\
97\end{array}$ & $\begin{array}{c}786 \\
39\end{array}$ & $\begin{array}{c}430 \\
1120\end{array}$ & $\begin{array}{c}37.8 \\
1.2\end{array}$ & $\begin{array}{c}840 \\
46\end{array}$ & $\begin{array}{l}290 \\
900\end{array}$ & $\begin{array}{l}49.5 \\
1.5\end{array}$ \\
\hline 300.0 & $\begin{array}{c}341 \\
17\end{array}$ & $\begin{array}{l}73 \\
98\end{array}$ & $\begin{array}{c}642 \\
37\end{array}$ & $\begin{array}{l}180 \\
650\end{array}$ & $\begin{array}{l}47.6 \\
1.4\end{array}$ & $\begin{array}{c}1000 \\
57\end{array}$ & $\begin{array}{l}100 \\
670\end{array}$ & $\begin{array}{c}100.0 \\
2.2\end{array}$ \\
\hline & & & \multicolumn{6}{|c|}{$m_{\mathrm{t}}=200 \mathrm{GeV}$} \\
\hline 200.0 & $\begin{array}{c}561 \\
9\end{array}$ & $\begin{array}{l}71 \\
96\end{array}$ & $\begin{array}{c}675 \\
15\end{array}$ & $\begin{array}{c}900 \\
1000\end{array}$ & $\begin{array}{c}22.5 \\
0.5\end{array}$ & $\begin{array}{c}336 \\
12\end{array}$ & $\begin{array}{l}170 \\
470\end{array}$ & $\begin{array}{c}26.0 \\
0.6\end{array}$ \\
\hline 250.0 & $\begin{array}{l}472 \\
21\end{array}$ & $\begin{array}{l}72 \\
97\end{array}$ & $\begin{array}{c}786 \\
39\end{array}$ & $\begin{array}{l}370 \\
900\end{array}$ & $\begin{array}{c}40.9 \\
1.3\end{array}$ & $\begin{array}{c}840 \\
46\end{array}$ & $\begin{array}{l}200 \\
660\end{array}$ & $\begin{array}{c}60.2 \\
1.8\end{array}$ \\
\hline 300.0 & $\begin{array}{c}341 \\
17\end{array}$ & $\begin{array}{l}73 \\
98\end{array}$ & $\begin{array}{c}642 \\
37\end{array}$ & $\begin{array}{l}150 \\
530\end{array}$ & $\begin{array}{c}51.9 \\
1.6\end{array}$ & $\begin{array}{c}1000 \\
57\end{array}$ & $\begin{array}{c}70 \\
500\end{array}$ & $\begin{array}{c}123.1 \\
2.6\end{array}$ \\
\hline
\end{tabular}




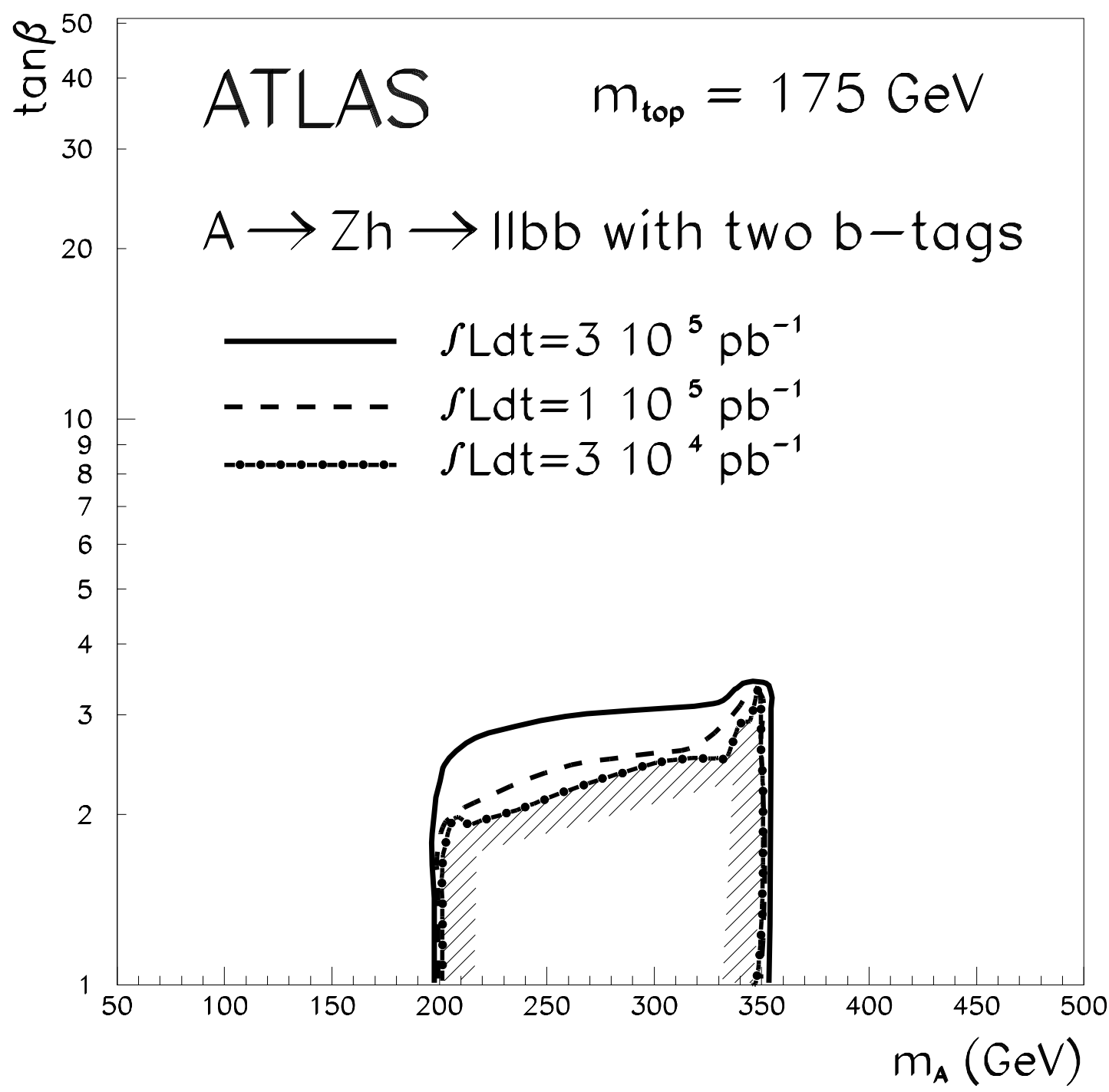

Figure 67: For integrated luminosities of $3 \cdot 10^{4} \mathrm{pb}^{-1}, 10^{5} \mathrm{pb}^{-1}$ and $3 \cdot 10^{5} \mathrm{pb}^{-1}$ and $m_{\mathrm{t}}=175 \mathrm{GeV}, 5 \sigma$-discovery contour curves for the $\mathrm{A} \rightarrow \mathrm{Zh} \rightarrow \ell \ell \mathrm{b} \overline{\mathrm{b}}$ channel in the $\left(m_{\mathrm{A}}, \tan \beta\right)$ plane. 


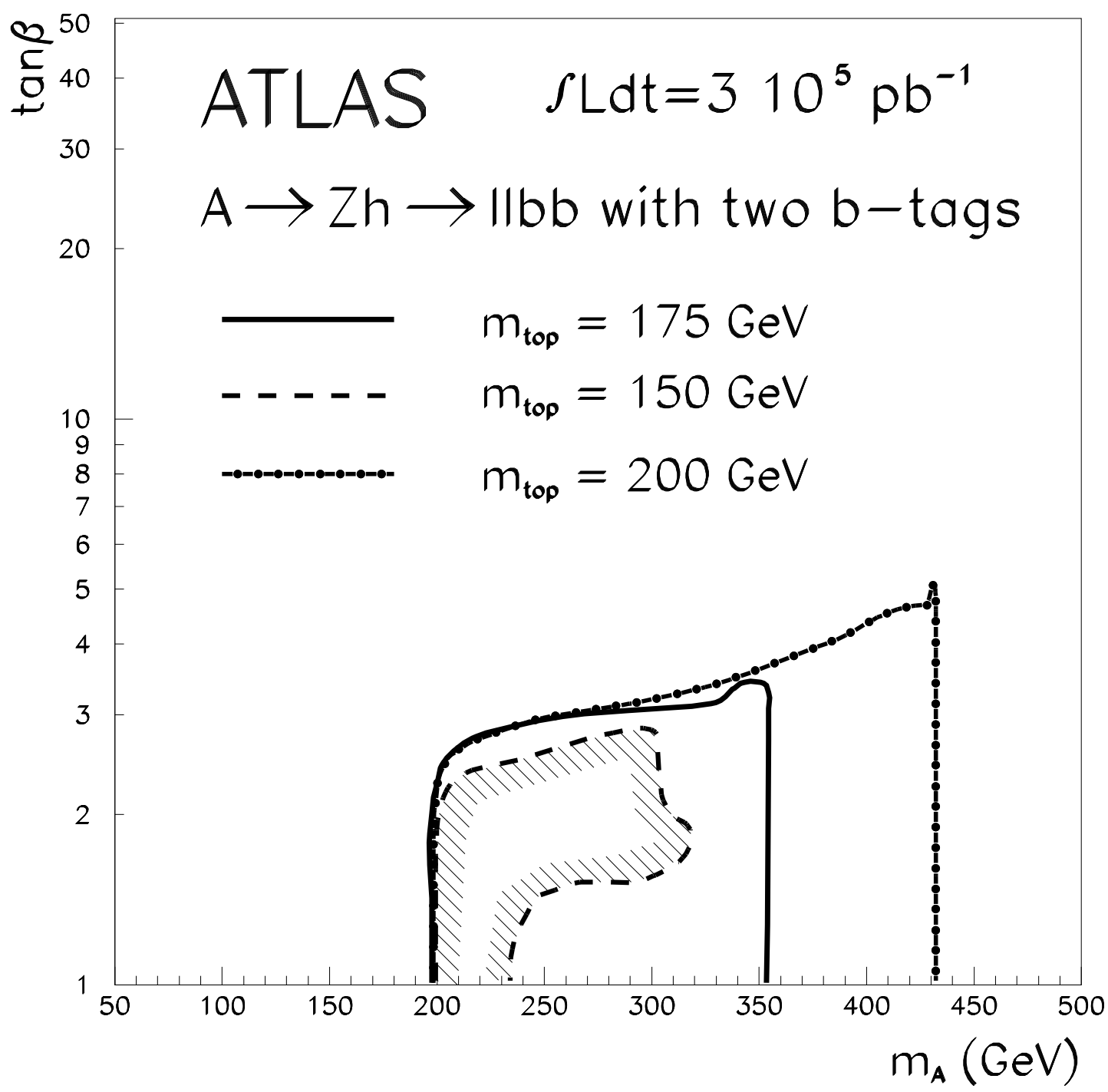

Figure 68: For $m_{\mathrm{t}}=150,175$ and $200 \mathrm{GeV}$ and an integrated luminosity of $3 \cdot 10^{5} \mathrm{pb}^{-1}, 5 \sigma$-discovery contour curves for the $\mathrm{A} \rightarrow \mathrm{Zh} \rightarrow \ell \ell \mathrm{b} \overline{\mathrm{b}}$ channel in the $\left(m_{\mathrm{A}}, \tan \beta\right)$ plane. 


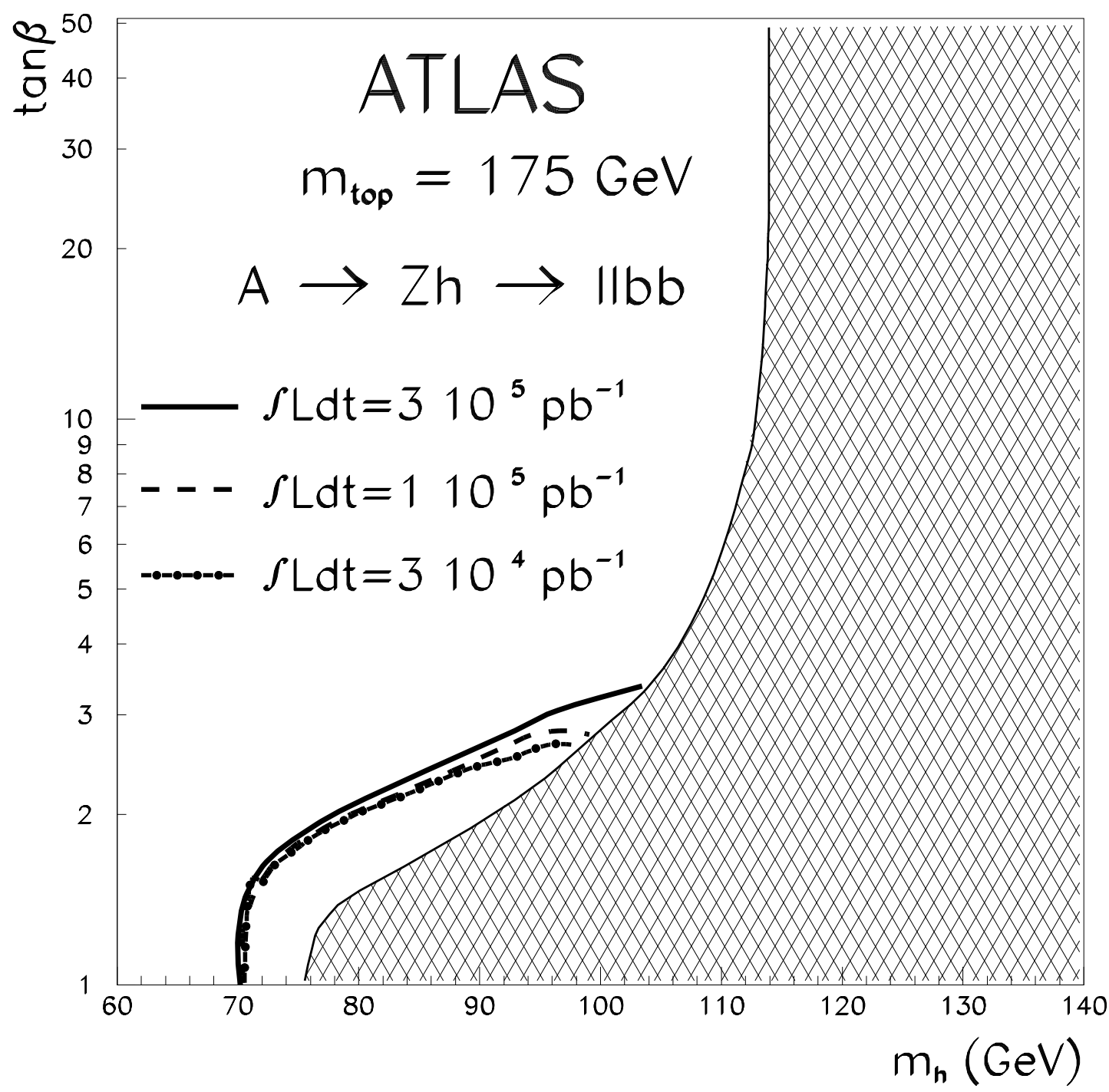

Figure 69: For integrated luminosities of $3 \cdot 10^{4} \mathrm{pb}^{-1}, 10^{5} \mathrm{pb}^{-1}$ and $3 \cdot 10^{5} \mathrm{pb}^{-1}$ and $m_{\mathrm{t}}=175 \mathrm{GeV}, 5 \sigma$-discovery contour curves for the $\mathrm{A} \rightarrow \mathrm{Zh} \rightarrow \ell \ell \mathrm{b} \overline{\mathrm{b}}$ channel in the $\left(m_{\mathrm{h}}, \tan \beta\right)$ plane. The cross-hatched area is theoretically excluded. 


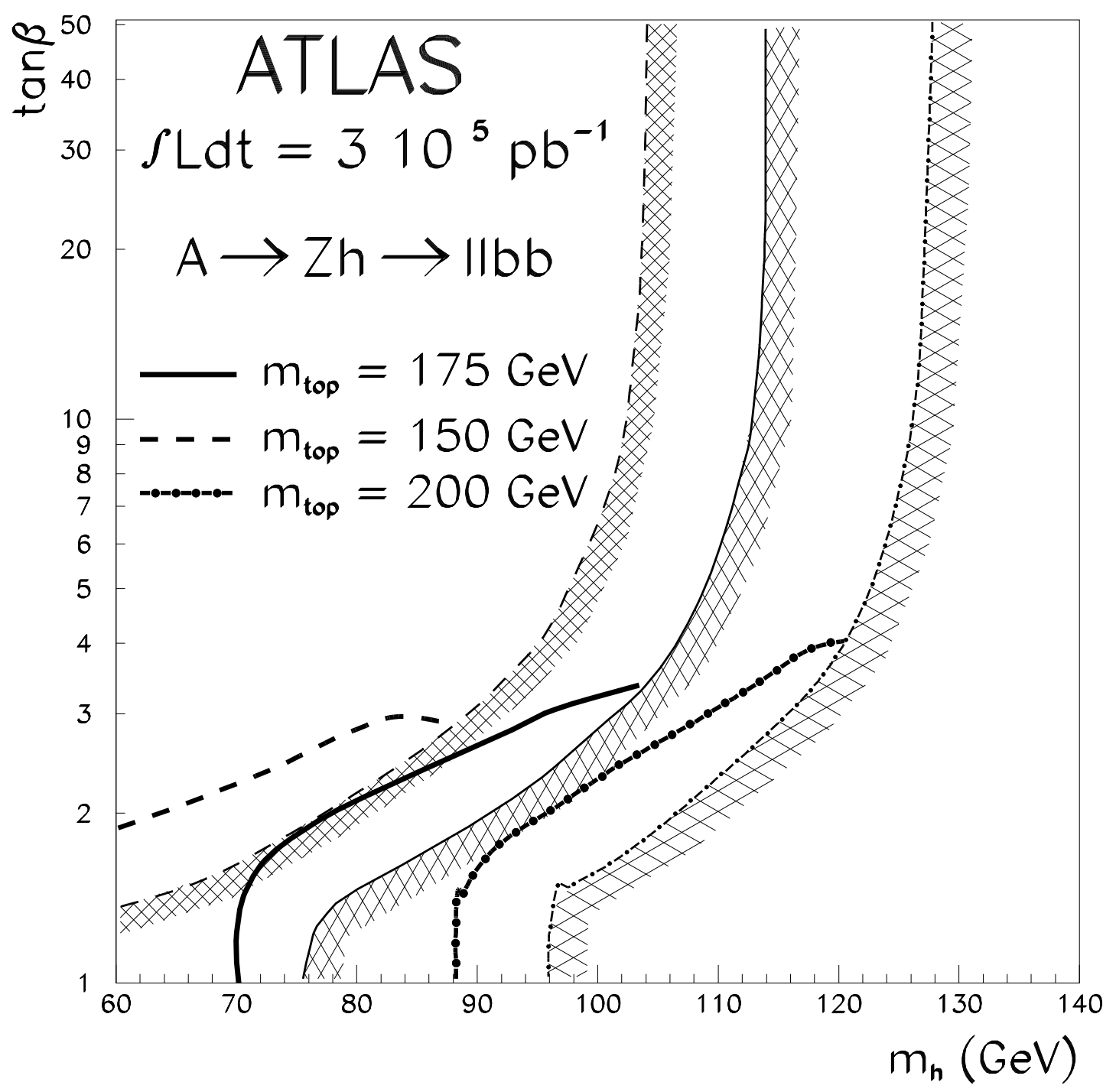

Figure 70: For $m_{\mathrm{t}}=150,175$ and $200 \mathrm{GeV}$ and an integrated luminosity of $3 \cdot 10^{5} \mathrm{pb}^{-1}, 5 \sigma$-discovery contour curves for the $\mathrm{A} \rightarrow \mathrm{Zh} \rightarrow \ell \ell \mathrm{b} \overline{\mathrm{b}}$ channel in the $\left(m_{\mathrm{h}}, \tan \beta\right)$ plane. The cross-hatched areas are theoretically excluded. 


\subsection{The $\mathrm{H}^{ \pm} \rightarrow \tau \nu$ channel}

Charged Higgs boson production at the LHC can occur through t $\bar{t}$ production followed by $\mathrm{t} \rightarrow \mathrm{H}^{+} \mathrm{b}$ decay or through Drell-Yan pair production. The latter is unfortunately much smaller in rate and much more difficult to extract from the huge QCD backgrounds. The results presented here are therefore based on the studies performed for the TP [26] with full simulation of $t \rightarrow \mathrm{H}^{+} \mathrm{b}$ decays and their dominant backgrounds.

This study concentrated on the search in $t \bar{t}$ events for an excess of $\tau$ leptons from $\mathrm{H}^{ \pm} \rightarrow \tau \nu$ decay with respect to the expected $\tau$-lepton rate from $\mathrm{W}^{ \pm} \rightarrow \tau \nu$ decay. The charged Higgs boson mass cannot be directly reconstructed, because several neutrinos are produced in the final states of interest.

Large samples of $t \bar{t}$ events can be triggered on by requiring one isolated high-p $\mathrm{p}_{\mathrm{T}}$ lepton within $|\eta|<2.5$. The additional requirement of at least three reconstructed jets with $\mathrm{p}_{\mathrm{T}}>20 \mathrm{GeV}$ and $|\eta|<2.5$, of which two are required to be tagged as b-jets, reduces the potentially large backgrounds from $W+$ jet and $b \bar{b}$ production to a level well below the $t \bar{t}$ signal itself. The dominant background is then the combinatorial background from fake and real $\tau$-leptons in $t \bar{t}$ events. The selection cuts enhance the right-handed $\tau$-lepton signal from $\mathrm{H}^{ \pm}$decays with respect to that from $\mathrm{W}$ decay, and select mostly single-prong $\tau$-decays. As for the case of the $\mathrm{H} / \mathrm{A} \rightarrow \tau \tau$ decays discussed in Section 5.4, $\tau$ identification is a key element in extracting a possible signal from the large combinatorial background from jets.

After the selection cuts and the $\tau$ identification criteria have been applied, $\mathrm{t} \rightarrow \mathrm{H}^{+} \mathrm{b}$ decays appear as final states with an excess of events with one isolated $\tau$-lepton compared to those with an additional isolated electron or muon. Table 39 gives, for $m_{\mathrm{t}}=150,175$ and $200 \mathrm{GeV}$, the expected $\tau$-lepton excess rates from $\mathrm{t} \rightarrow \mathrm{H}^{+} \mathrm{b}$ decay as a function of $m_{\mathrm{H}^{ \pm}}$as well as the summed background rates from real and fake $\tau$-leptons. As in the case of $\mathrm{H} / \mathrm{A} \rightarrow \tau \tau$ decays, these results were obtained from full simulation of the signal and background processes. As an example, for $m_{\mathrm{t}}=175 \mathrm{GeV}, m_{\mathrm{H}^{ \pm}}=130 \mathrm{GeV}$ and $\tan \beta=6$, an excess of $\sim 1000 \tau$-leptons is expected from the charged Higgs boson signal, above a background of $\sim 3000 \tau$-leptons from $\mathrm{W}$ decay, and of $\sim 4000$ fake $\tau$-leptons.

When measuring such an excess, systematic uncertainties have to be taken into account. They arise mainly from the imperfect knowledge of the $\tau$-lepton efficiency and of the amount of fake $\tau$-leptons present in the final sample. They were assumed to be $\sim \pm 3 \%$ from past experience [27], and added to the statistical uncertainty to obtain the significances shown in Table 39. These systematic uncertainties dominate the overall uncertainty, and the sensitivity to a charged Higgs boson signal would not improve significantly with integrated luminosity unless increased statistics would result in improved systematic uncertainties. It is important to recall, as mentioned in Section 3.4.1, that these results do not take into account recent calculations [9], which in-

clude possible decays of the charged Higgs boson to SUSY particles and show 
that the $\mathrm{H}^{+} \rightarrow \tau \nu$ branching ratio may in some cases decrease significantly for low values of $\tan \beta$.

Figs. 71 and 72 show the expected $5 \sigma$-discovery contour curves for this channel in the $\left(m_{\mathrm{A}}, \tan \beta\right)$ and $\left(m_{\mathrm{h}}, \tan \beta\right)$ planes as a function of $m_{\mathrm{t}}$ for an integrated luminosity of $3 \cdot 10^{4} \mathrm{pb}^{-1}$. A signal from charged Higgs boson production in $\mathrm{t} \overline{\mathrm{t}}$ decays would be observed for all values of $m_{\mathrm{H}^{ \pm}}$below the kinematical limit of $\sim m_{\mathrm{t}}-20 \mathrm{GeV}$ over most of the $\tan \beta$-range. For moderate values of $\tan \beta$, for which the expected signal rates are lowest, the accessible values of $m_{\mathrm{H}^{ \pm}}$are lower than this kinematical limit by $\sim 20 \mathrm{GeV}$. This effect becomes more pronounced as $m_{\mathrm{t}}$ increases, due to the decrease in the $t \bar{t}$ production cross-section. One can note finally that, as for the $\mathrm{H} / \mathrm{A} \rightarrow \tau \tau$ channel, the fraction of parameter space covered by the $\mathrm{H}^{ \pm} \rightarrow \tau \nu$ channel in the $\left(m_{\mathrm{h}}, \tan \beta\right)$ plane is much larger than in the standard $\left(m_{\mathrm{A}}, \tan \beta\right)$ plane.

Table 39: Observability of the $\mathrm{H}^{ \pm} \rightarrow \tau \nu$ channel at low luminosity. The $\sigma \times B R$ values and the expected numbers of signal and background events are given for the production of one or two charged Higgs bosons in t $\overline{\mathrm{t}}$ decay and for $\tan \beta=5$.

\begin{tabular}{|c|c||c|c|c|c|}
\hline \multicolumn{2}{|c||}{} & \multicolumn{4}{c|}{ Low luminosity } \\
\cline { 4 - 7 } & \multicolumn{4}{c|}{$3 \cdot 10^{4} \mathrm{pb}^{-1}$} \\
\hline $\begin{array}{c}m_{\mathrm{H}^{ \pm}} \\
(\mathrm{GeV})\end{array}$ & $\begin{array}{c}\sigma \times B R \\
(\mathrm{fb})\end{array}$ & Signal & Background & Error & Signif. \\
\hline \hline \multicolumn{2}{|l||}{} & \multicolumn{4}{|c|}{$m_{\mathrm{t}}=150 \mathrm{GeV}$} \\
\hline \hline 110.0 & 32.7 & 4290 & 9850 & 318 & 13.5 \\
130.0 & 10.2 & 1210 & 7900 & 255 & 4.7 \\
\hline \hline \multicolumn{2}{|l||}{} & \multicolumn{4}{|c|}{$m_{\mathrm{t}}=175 \mathrm{GeV}$} \\
\hline \hline 110.0 & 23.3 & 3050 & 7020 & 233 & 13.1 \\
130.0 & 13.1 & 1550 & 7170 & 234 & 6.6 \\
150.0 & 4.8 & 380 & 9120 & 290 & 1.3 \\
\hline \hline \multicolumn{2}{|c||}{} & \multicolumn{4}{c|}{$m_{\mathrm{t}}=200 \mathrm{GeV}$} \\
\hline \hline 110.0 & 15.4 & 2010 & 4350 & 153 & 13.1 \\
130.0 & 10.7 & 1260 & 4710 & 161 & 7.8 \\
150.0 & 6.3 & 490 & 6330 & 207 & 2.4 \\
\hline
\end{tabular}




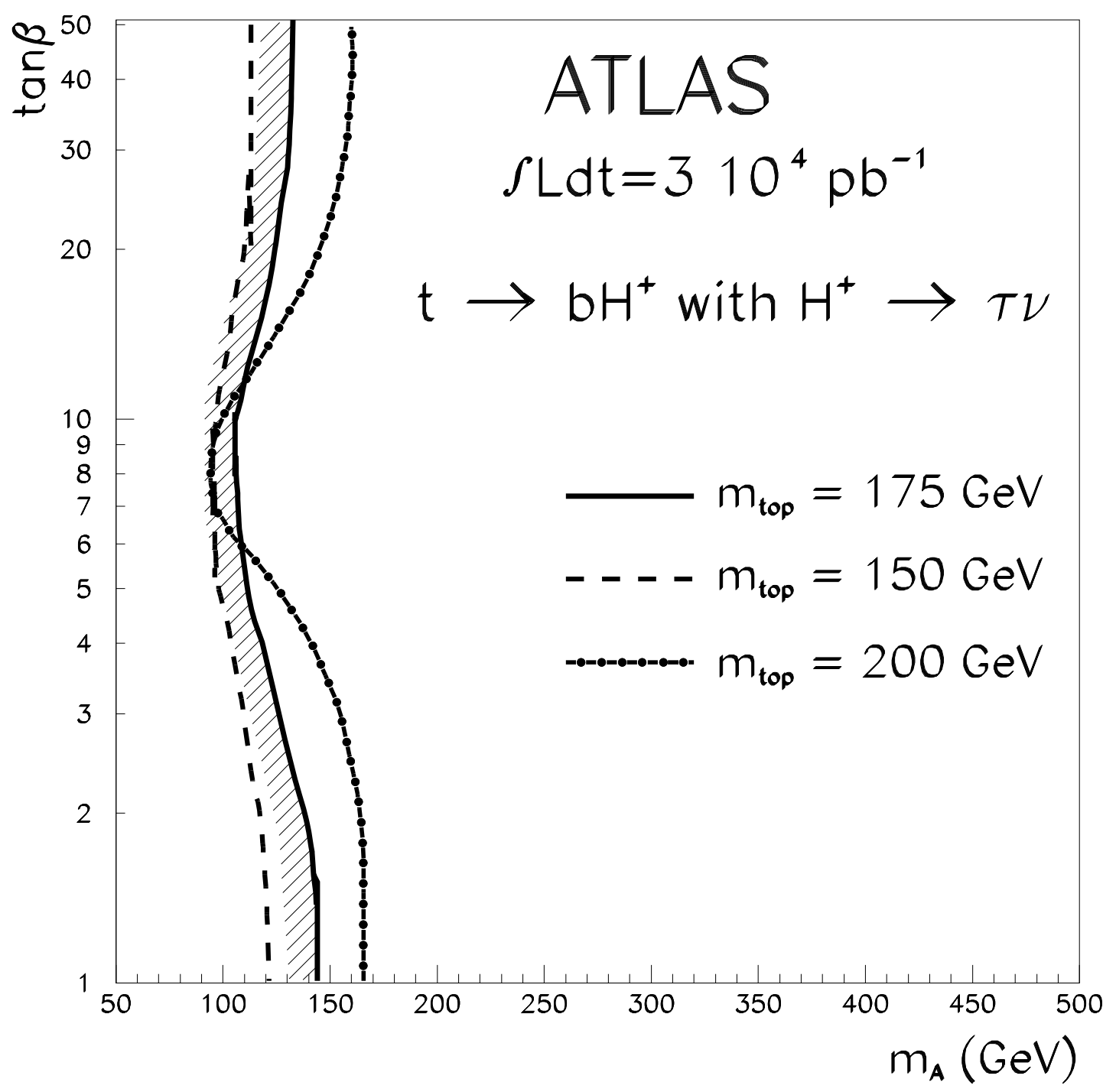

Figure 71: For $m_{\mathrm{t}}=150,175$ and $200 \mathrm{GeV}$ and an integrated luminosity of $3 \cdot 10^{4} \mathrm{pb}^{-1}, 5 \sigma$-discovery contour curves for the $\mathrm{H}^{ \pm} \rightarrow \tau \nu$ channel in the $\left(m_{\mathrm{A}}, \tan \beta\right)$ plane. 


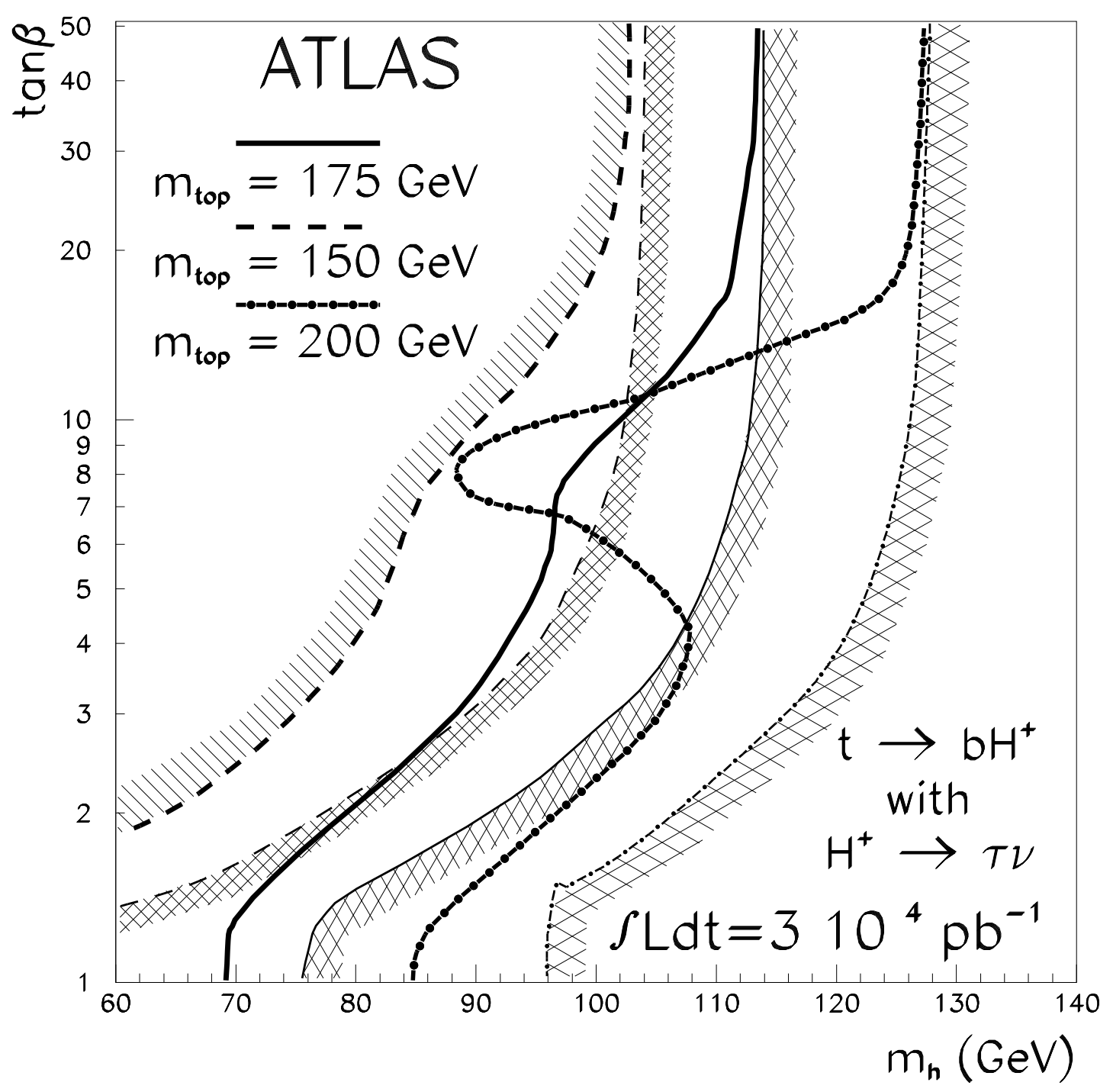

Figure 72: For $m_{\mathrm{t}}=150,175$ and $200 \mathrm{GeV}$ and an integrated luminosity of $3 \cdot 10^{4} \mathrm{pb}^{-1}, 5 \sigma$-discovery contour curves for the $\mathrm{H}^{ \pm} \rightarrow \tau \nu$ channel in the $\left(m_{\mathrm{h}}, \tan \beta\right)$ plane. The cross-hatched areas are theoretically excluded. 


\section{Conclusions and outlook}

This Section is mainly devoted to presenting the results, as discussed in Section 5 for each signal channel separately, in a combined way, in order to obtain a more global perspective on the LHC discovery potential in the MSSM Higgs sector. It also discusses possible improvements and extensions of the studies done to date. The Figures presented below combine together the $5 \sigma$-discovery contour curves presented separately for each channel in Section 5 for different scenarios, i.e. for integrated luminosities of $3 \cdot 10^{4} \mathrm{pb}^{-1}$ or $3 \cdot 10^{5} \mathrm{pb}^{-1}$ and for the ATLAS experiment alone or the ATLAS and CMS experiments combined, under the assumptions described in Section 4. Colour PostScript versions of these Figures and of those shown in Section 5 are available on WWW ${ }^{9}$.

Figs. 73 (ATLAS) and 74 (ATLAS + CMS) show the combined $5 \sigma$-discovery contour curves for $m_{\mathrm{t}}=175 \mathrm{GeV}$ and an integrated luminosity of $3 \cdot 10^{4} \mathrm{pb}^{-1}$, representative of the integrated luminosity at the LHC after three years of operation. Figs. 75 and 76 show the corresponding curves for an integrated luminosity of $3 \cdot 10^{5} \mathrm{pb}^{-1}$, representative of the ultimate integrated luminosity at the LHC after about 10 years of operation. All these Figures also display the LEP2 discovery potential for the upgraded machine energy of $192 \mathrm{GeV}$ and for an integrated luminosity of $150 \mathrm{pb}^{-1}$ per experiment [8]. Although some theoretical arguments tend to favour small values of $\tan \beta$, the impression given by the choice of a logarithmic scale for $\tan \beta$ can be misleading, and Figs. 77 and 78 display the curves of Figs. 74 and 76 with a linear scale for $\tan \beta$. The importance of the combined $h \rightarrow \gamma \gamma$ channel and of the $\mathrm{H} / \mathrm{A} \rightarrow \tau \tau$ channel at the LHC thus appears more clearly.

As discussed in Section 2, the contour curves in these Figures can be affected significantly by changes in some of the parameters in the MSSM model. The most significant one is the value of $m_{\mathrm{t}}$, as illustrated with a log- (resp. linear) scale for $\tan \beta$ by Figs. 79 for ATLAS, 80 (resp. 83) for ATLAS+CMS and for $m_{\mathrm{t}}=150 \mathrm{GeV}$, and Figs. 81 for ATLAS, 82 (resp. 84) for AT$\mathrm{LAS}+\mathrm{CMS}$ and for $m_{\mathrm{t}}=200 \mathrm{GeV}$. As $m_{\mathrm{t}}$ increases, the LEP2 discovery potential decreases, due to the larger value of $m_{\mathrm{h}}$ at any given point in the $\left(m_{\mathrm{A}}, \tan \beta\right)$ plane, whereas the LHC discovery potential increases significantly for some channels $\left(\mathrm{h} \rightarrow \gamma \gamma\right.$ and $\mathrm{H} \rightarrow \mathrm{ZZ}^{(*)} \rightarrow 4 \ell$ ), remains unchanged for others $(\mathrm{H} / \mathrm{A} \rightarrow \tau \tau$ and $\mathrm{A} \rightarrow \mathrm{Zh} \rightarrow \ell \ell \mathrm{b} \overline{\mathrm{b}})$, or corresponds to somewhat different regions of the parameter space for the remaining channels. Nevertheless, the overall picture remains the same:

- the LEP2 discovery potential corresponds to $\sim 10-20 \%$ of the parameter space in a linear $\left(m_{\mathrm{A}}, \tan \beta\right)$ plane. In most cases, the discovery of a Higgs boson at LEP2 would not in itself allow any discrimination between the SM case and the MSSM case;

\footnotetext{
${ }^{9} \mathrm{http:://atlasinfo.cern.ch/Atlas/GROUPS/PHYSICS/NOTES/note74}$
} 
- with a modest integrated luminosity of $3 \cdot 10^{4} \mathrm{pb}^{-1}$, the LHC discovery potential corresponds to $\sim 80 \%$ of the parameter space. For $80 \%$ to $90 \%$ of the cases, the discovery of a Higgs boson at the LHC would allow discrimination between the SM case and the MSSM case;

- with the very high integrated luminosity of $3 \cdot 10^{5} \mathrm{pb}^{-1}$, the LHC discovery potential corresponds to the whole parameter space. For almost all cases, the experiments would be able to distinguish between the SM case and the MSSM case. In Fig. 76, the region with $m_{\mathrm{A}}>250 \mathrm{GeV}$ and $4<\tan \beta<5-10$ is only covered by the $h \rightarrow \gamma \gamma$ channel. However, as discussed below, $h \rightarrow b \bar{b}$ decays from SUSY particle decays should be observable above background in this region for many cases, thus providing a direct evidence for SUSY. In the case of the simultaneous discovery of light $\mathrm{h}$ - and A-bosons at LEP2, essentially only the charged Higgs boson would be seen directly in top-quark decays at the LHC. In the more likely case of the discovery of one light h-boson at LEP2, several Higgs bosons would then be observed at the LHC;

- more generally, all three neutral Higgs bosons would be discovered at the LHC over $\sim 60 \%$ of the parameter space, i.e. for $m_{\mathrm{A}}>160 \mathrm{GeV}$, but over most of this region the $\mathrm{H}$ - and $\mathrm{A}$-bosons are degenerate in mass and would be very difficult to separate. Over $\sim 10 \%$ of the parameter space, i.e. for $\tan \beta>2$ and $90<m_{\mathrm{A}}<130 \mathrm{GeV}$, the two heavy neutral Higgs bosons and the charged Higgs boson would be discovered at the $\mathrm{LHC}$;

- over $\sim 5 \%$ of the parameter space, i.e. for $130<m_{\mathrm{A}}<160 \mathrm{GeV}$ and $\tan \beta>3$, only the $\mathrm{H} / \mathrm{A} \rightarrow \tau \tau$ channel seems to be observable at the LHC at this stage. However, as can be seen from Fig. 74, the Wh channel with $\mathrm{W} \rightarrow \ell \nu$ and $\mathrm{h} \rightarrow \mathrm{b} \overline{\mathrm{b}}$ decay provides sensitivity in this region for values of $\tan \beta$ as high as $\sim 5$ for an integrated luminosity of $3 \cdot 10^{4} \mathrm{pb}^{-1}$. Work is in progress to assess the observability of this channel at high luminosity, but also to determine whether the $t \bar{t} h$ channel could be useful to improve the sensitivity even further in this region of parameter space;

- the various channels described in Section 5 have also been studied for values of $\tan \beta$ smaller than 1 . Even if such values are disfavoured for theoretical reasons, it is important to assess the experimental sensitivity, and each channel was studied for $0.3<\tan \beta<2$ and for $m_{\mathrm{t}}=175 \mathrm{GeV}$, as shown in Figs. 85 to 92 , for ATLAS with an integrated luminosity of $3 \cdot 10^{5} \mathrm{pb}^{-1}$. These Figures show that, in contrast to LEP2, which has very little sensitivity to values of $\tan \beta$ below $\sim 0.8$, the sensitivity at LHC is quite good for most channels of interest in this region of very low values of $\tan \beta$; 


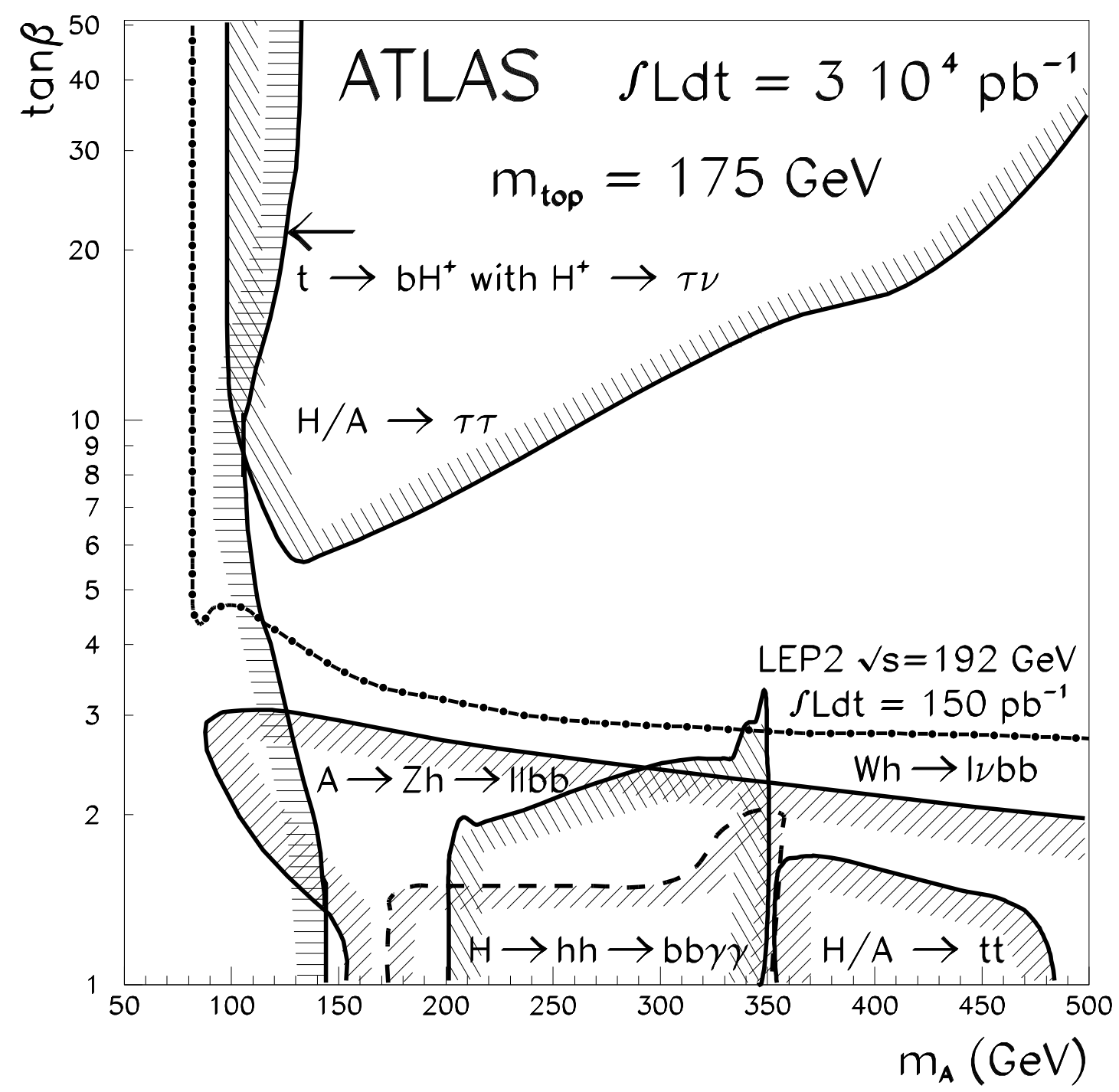

Figure 73: For $m_{\mathrm{t}}=175 \mathrm{GeV}$ and an integrated luminosity of $3 \cdot 10^{4} \mathrm{pb}^{-1}$, ATLAS $5 \sigma$-discovery contour curves in the $\left(m_{\mathrm{A}}, \tan \beta\right)$ plane for all Higgs boson signals discussed in Section 5. 


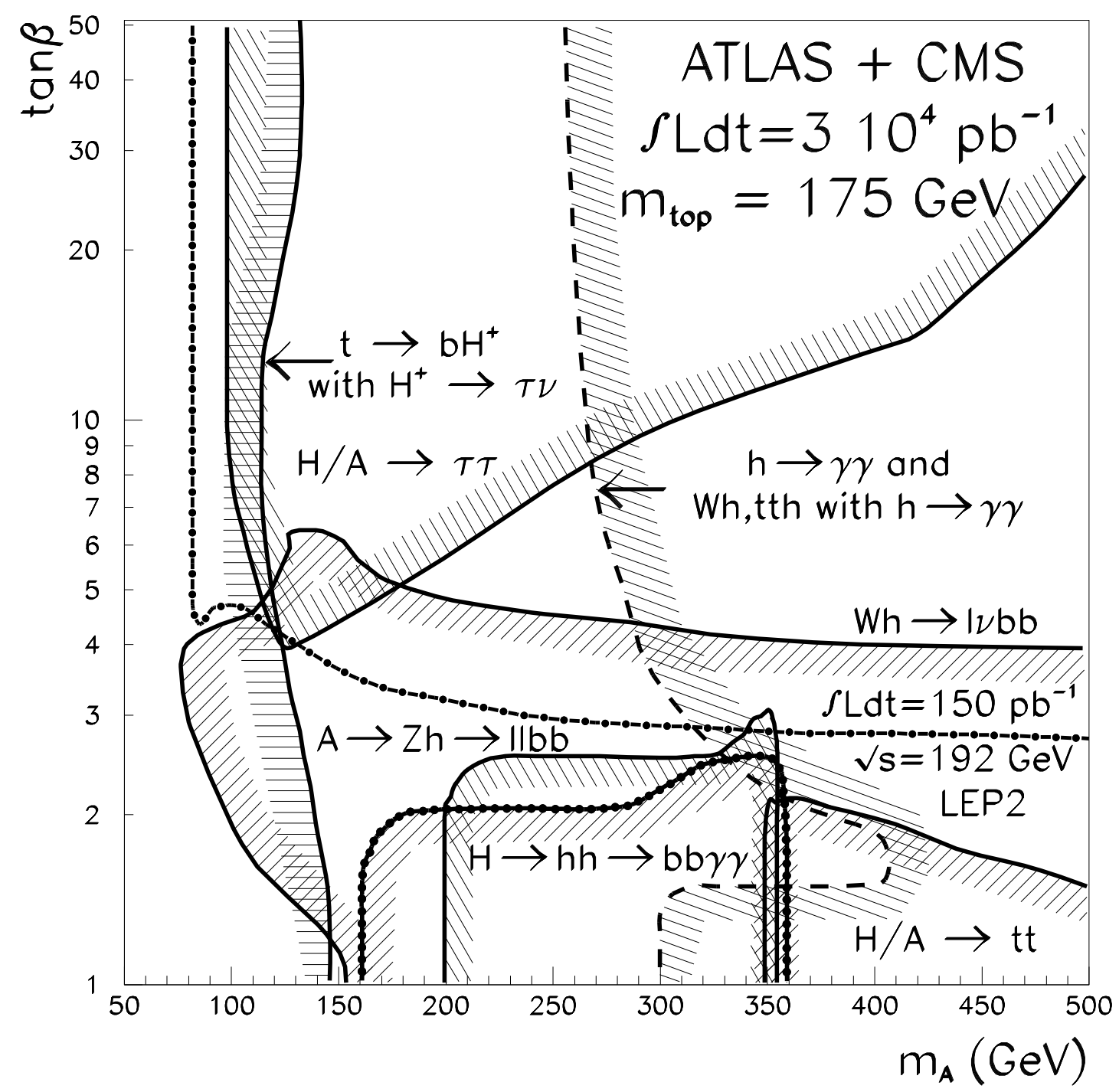

Figure 74: For $m_{\mathrm{t}}=175 \mathrm{GeV}$ and an integrated luminosity of $3 \cdot 10^{4} \mathrm{pb}^{-1}$, combined ATLAS+CMS $5 \sigma$-discovery contour curves in the $\left(m_{\mathrm{A}}, \tan \beta\right)$ plane for all Higgs boson signals discussed in Section 5. 


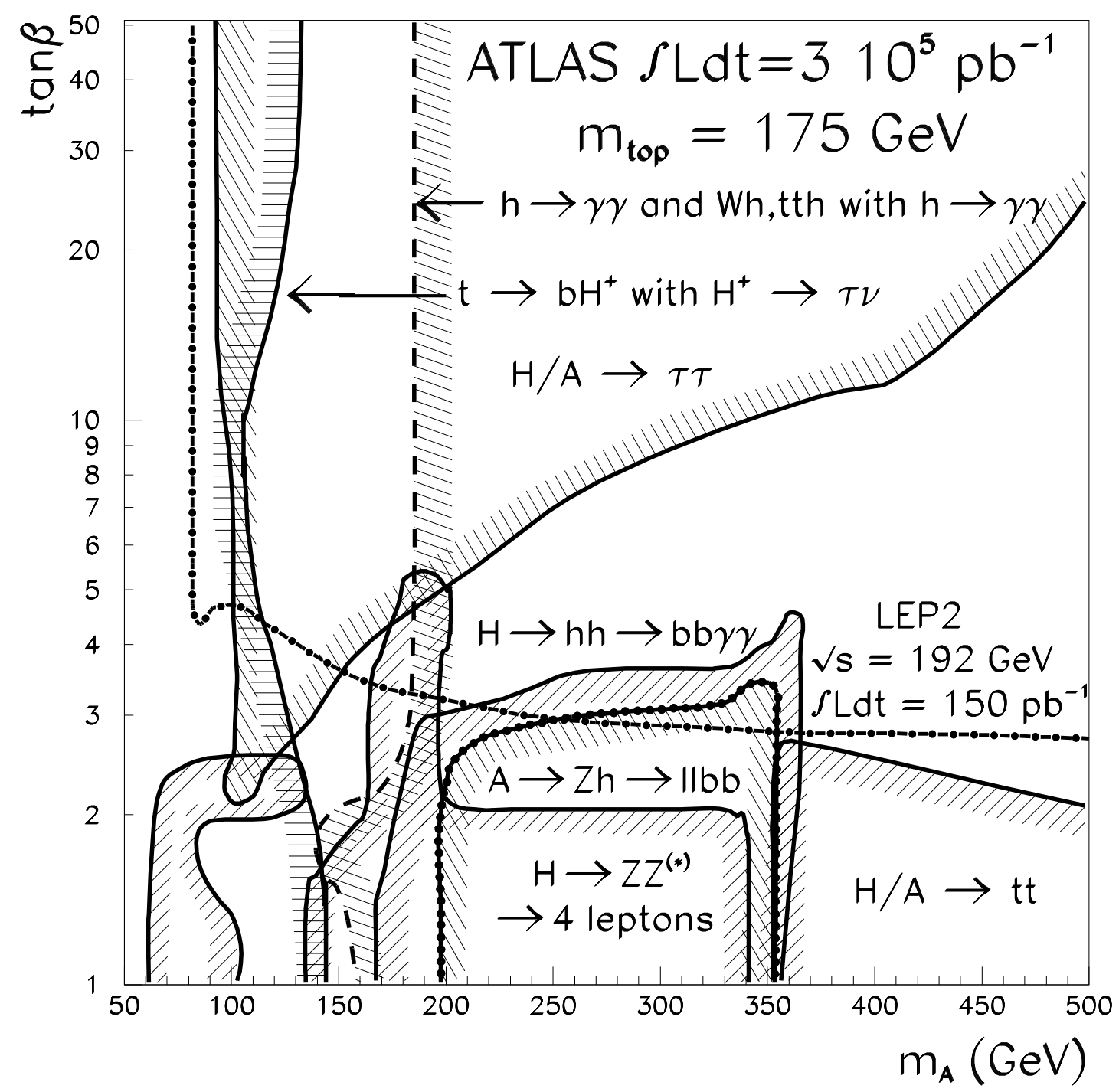

Figure 75: For $m_{\mathrm{t}}=175 \mathrm{GeV}$ and an integrated luminosity of $3 \cdot 10^{5} \mathrm{pb}^{-1}$, ATLAS $5 \sigma$-discovery contour curves in the $\left(m_{\mathrm{A}}, \tan \beta\right)$ plane for all Higgs boson signals discussed in Section 5. 


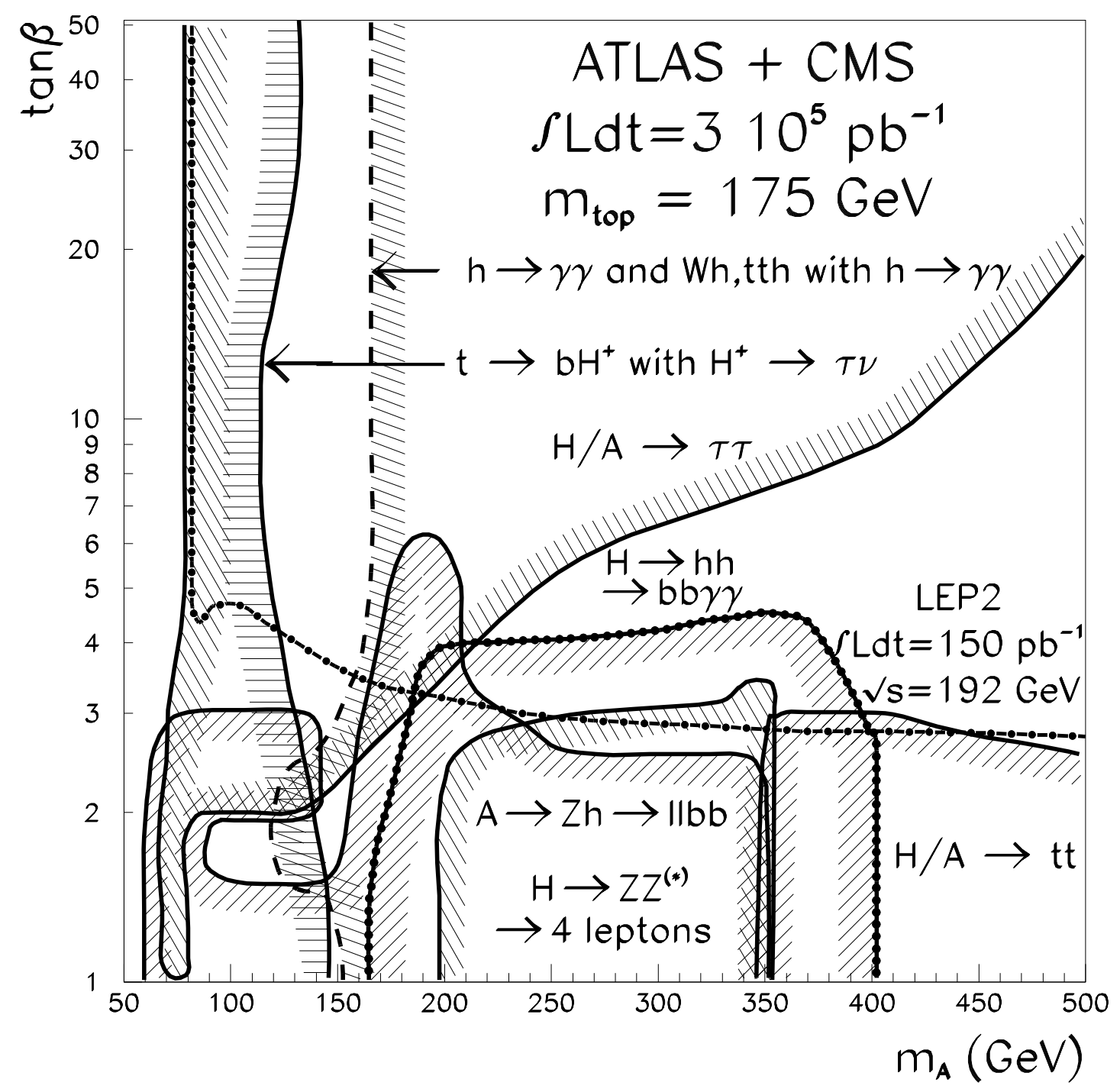

Figure 76: For $m_{\mathrm{t}}=175 \mathrm{GeV}$ and an integrated luminosity of $3 \cdot 10^{5} \mathrm{pb}^{-1}$, combined ATLAS+CMS $5 \sigma$-discovery contour curves in the $\left(m_{\mathrm{A}}, \tan \beta\right)$ plane for all Higgs boson signals discussed in Section 5. 


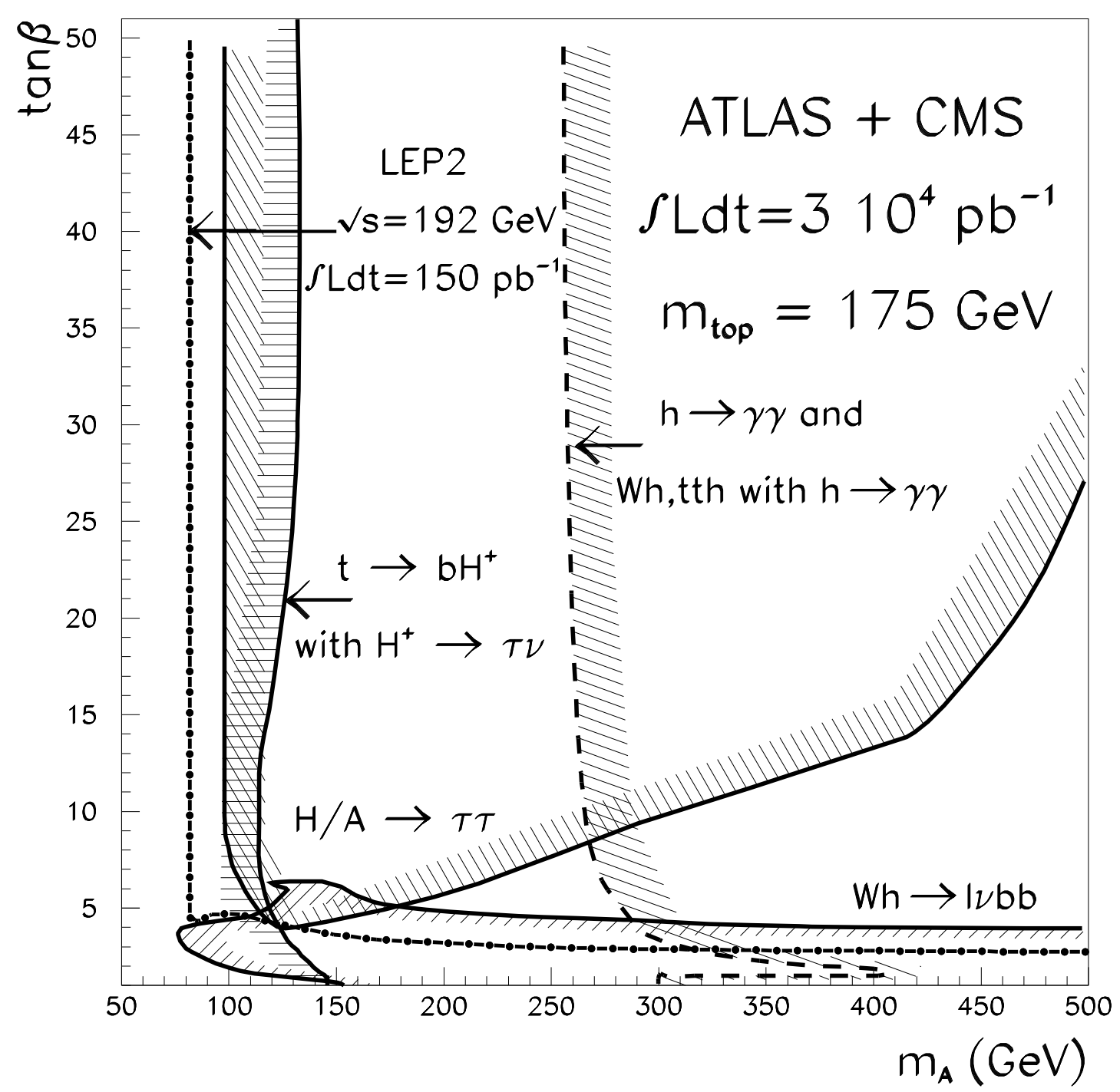

Figure 77: Same as Fig. 74 with a linear scale for $\tan \beta$. 


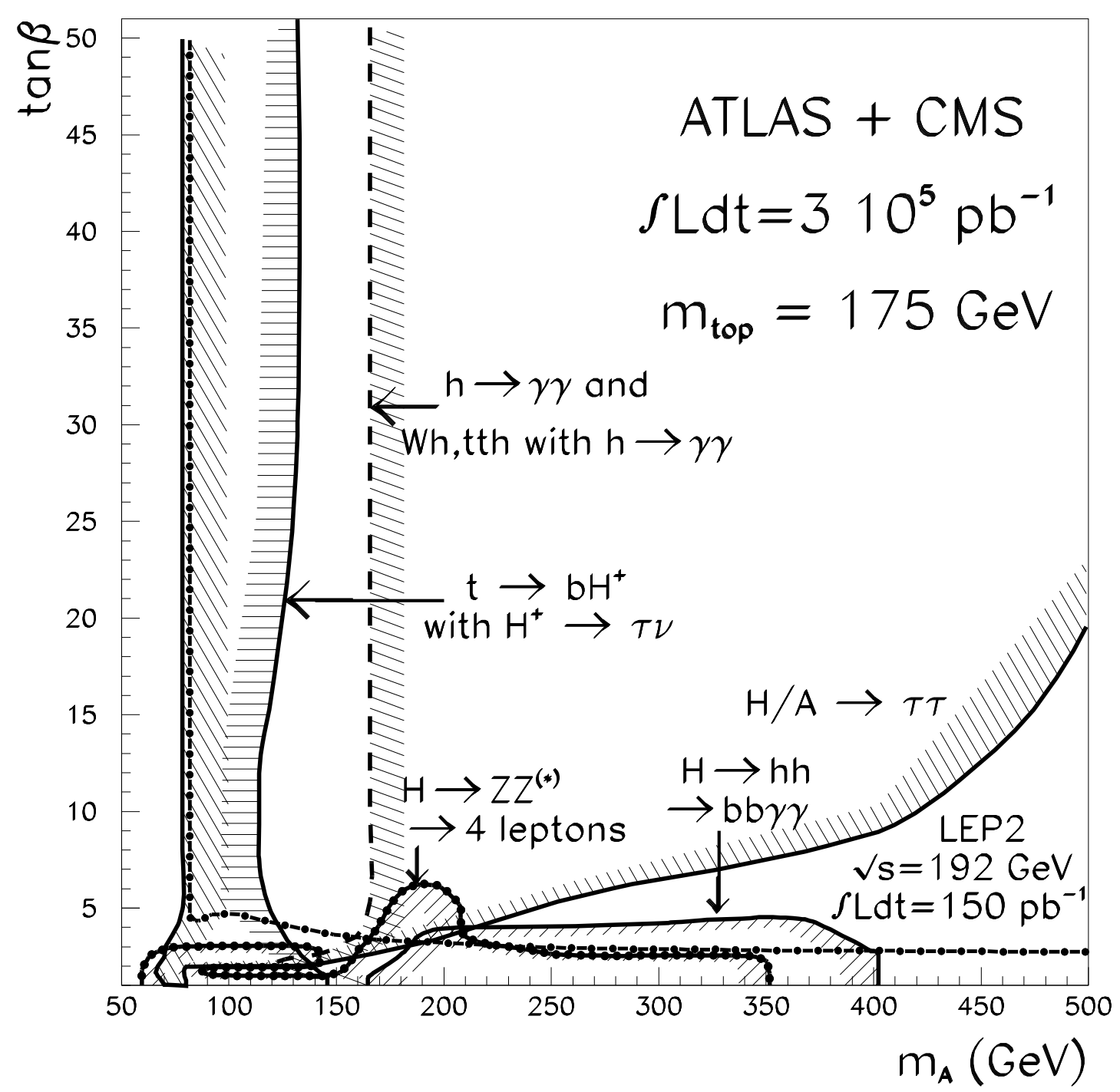

Figure 78: Same as Fig. 76 with a linear scale for $\tan \beta$. 


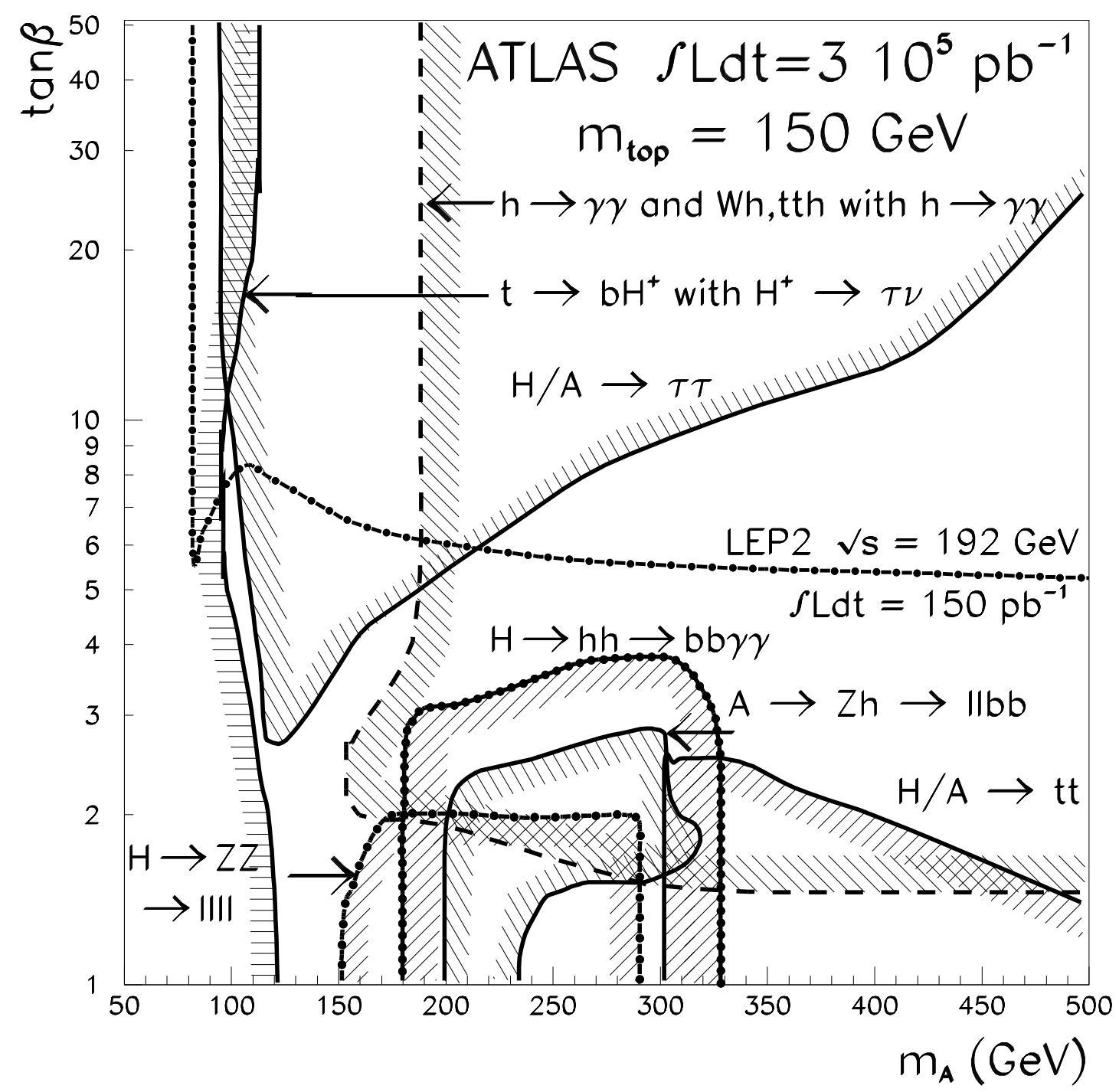

Figure 79: For $m_{\mathrm{t}}=150 \mathrm{GeV}$ and an integrated luminosity of $3 \cdot 10^{5} \mathrm{pb}^{-1}$, ATLAS $5 \sigma$-discovery contour curves in the $\left(m_{\mathrm{A}}, \tan \beta\right)$ plane for all Higgs boson signals discussed in Section 5. 


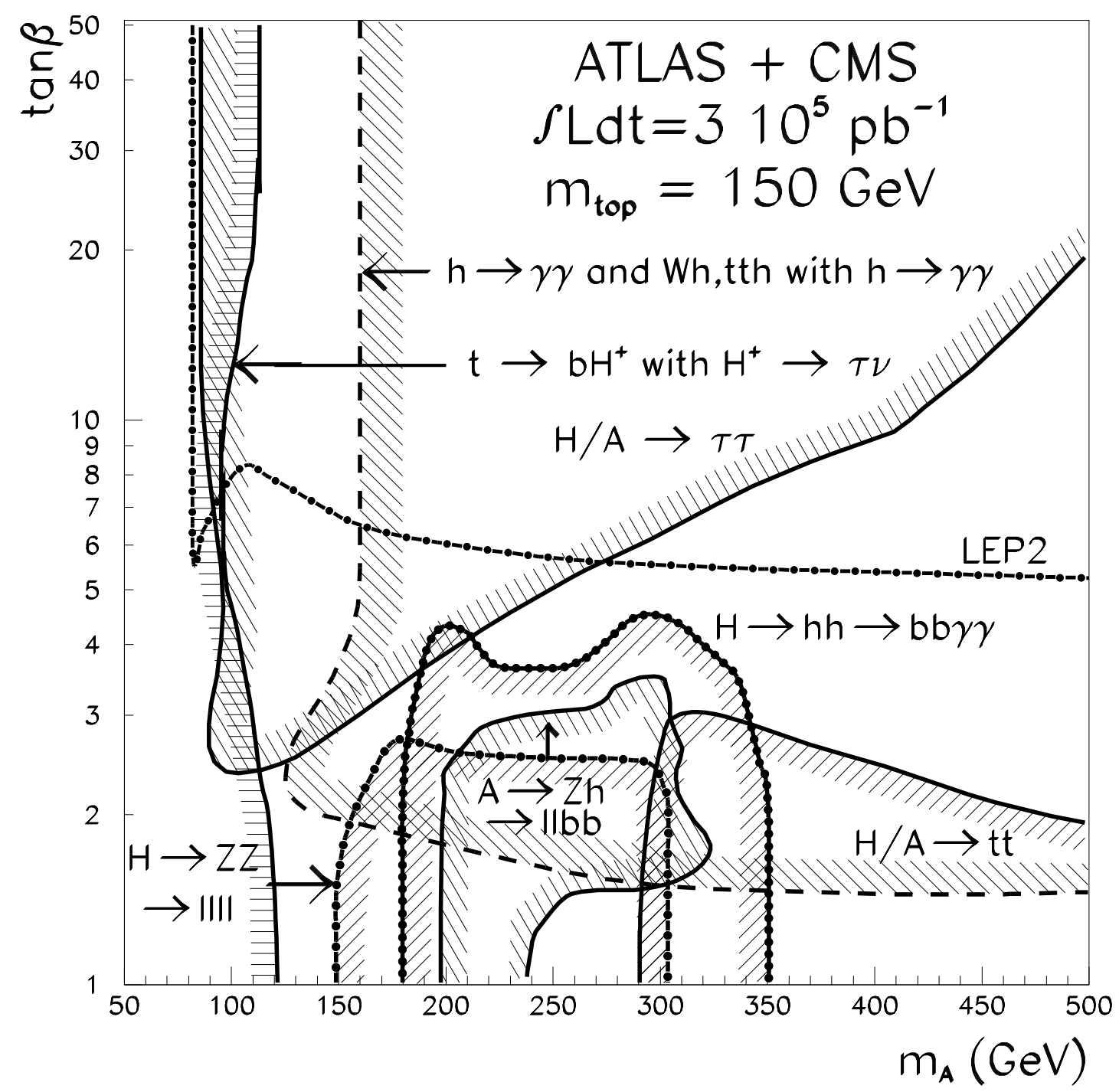

Figure 80: For $m_{\mathrm{t}}=150 \mathrm{GeV}$ and an integrated luminosity of $3 \cdot 10^{5} \mathrm{pb}^{-1}$, combined ATLAS+CMS $5 \sigma$-discovery contour curves in the $\left(m_{\mathrm{A}}, \tan \beta\right)$ plane for all Higgs boson signals discussed in Section 5. 


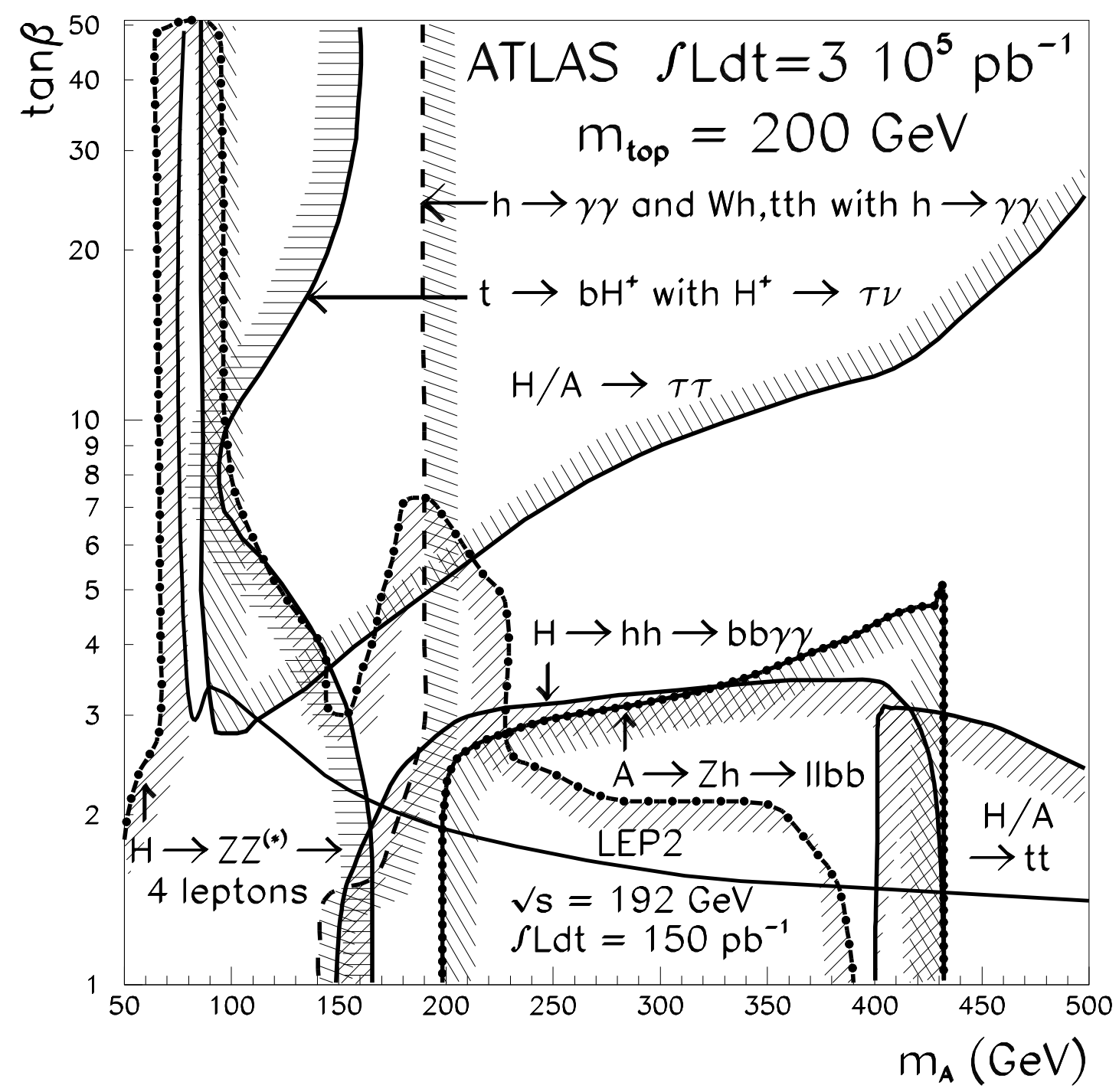

Figure 81: For $m_{\mathrm{t}}=200 \mathrm{GeV}$ and an integrated luminosity of $3 \cdot 10^{5} \mathrm{pb}^{-1}$, ATLAS $5 \sigma$-discovery contour curves in the $\left(m_{\mathrm{A}}, \tan \beta\right)$ plane for all Higgs boson signals discussed in Section 5. 


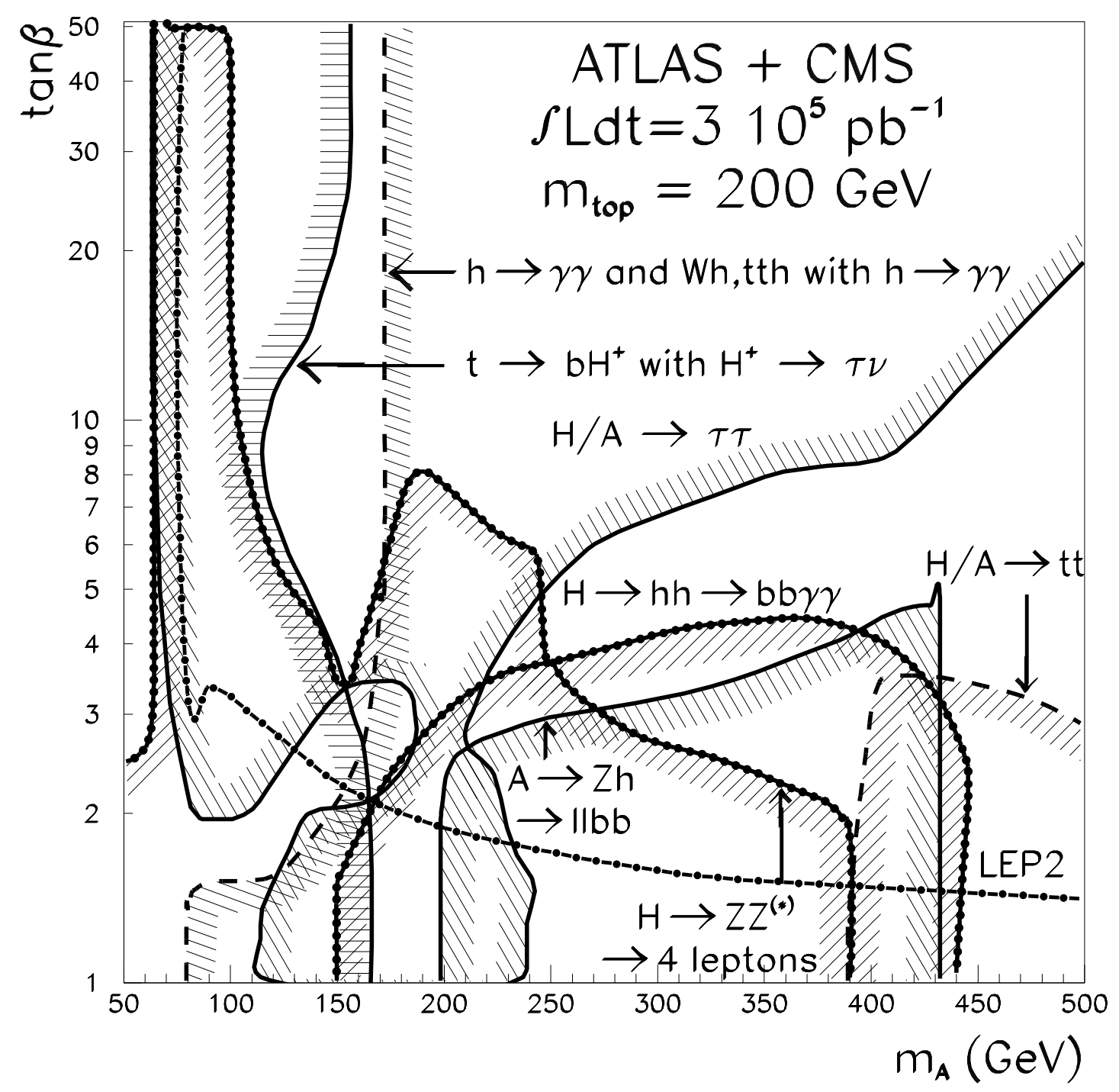

Figure 82: For $m_{\mathrm{t}}=200 \mathrm{GeV}$ and an integrated luminosity of $3 \cdot 10^{5} \mathrm{pb}^{-1}$, combined ATLAS+CMS $5 \sigma$-discovery contour curves in the $\left(m_{\mathrm{A}}, \tan \beta\right)$ plane for all Higgs boson signals discussed in Section 5. 


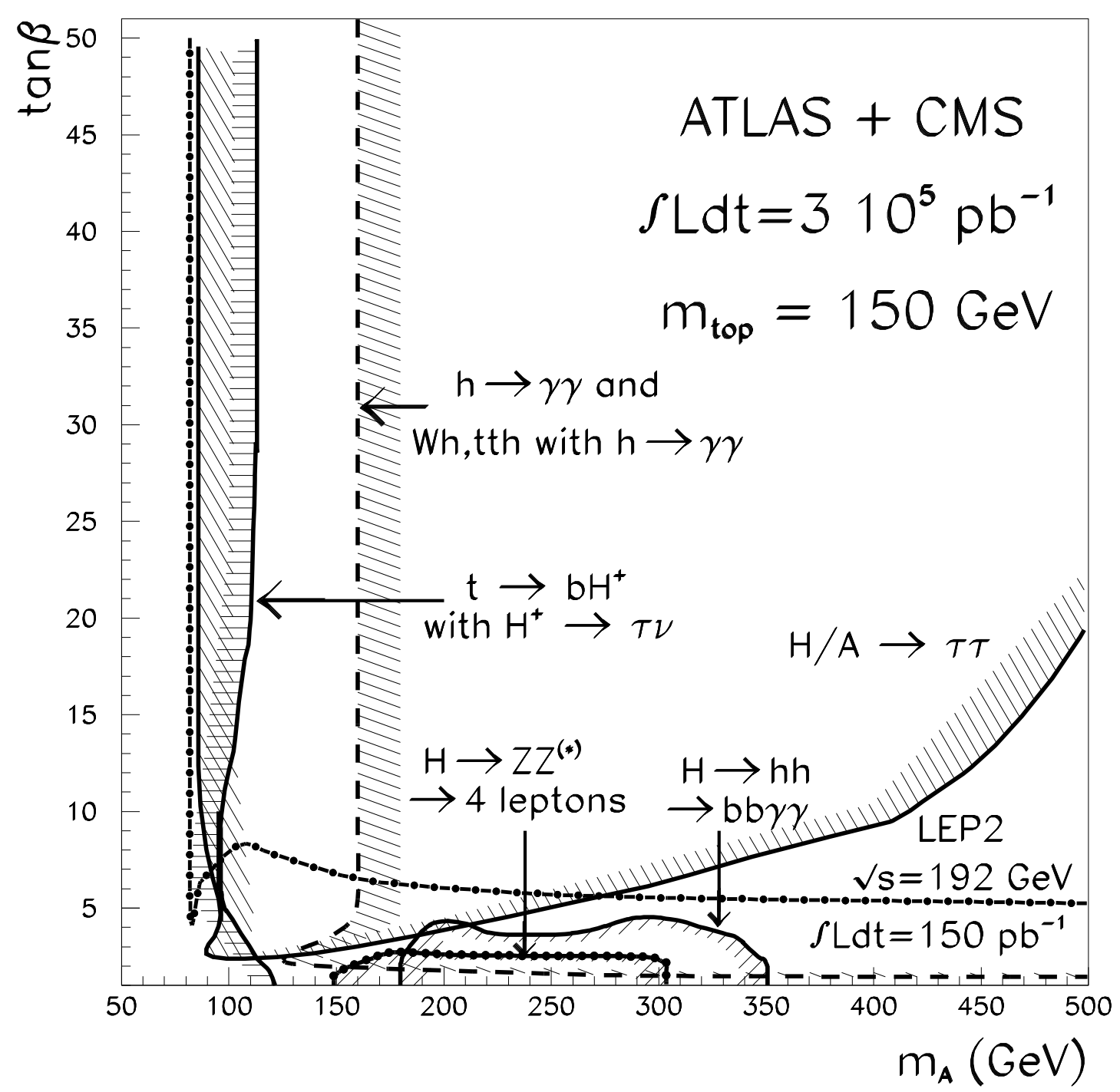

Figure 83: Same as Fig. 80 with a linear scale for $\tan \beta$. 


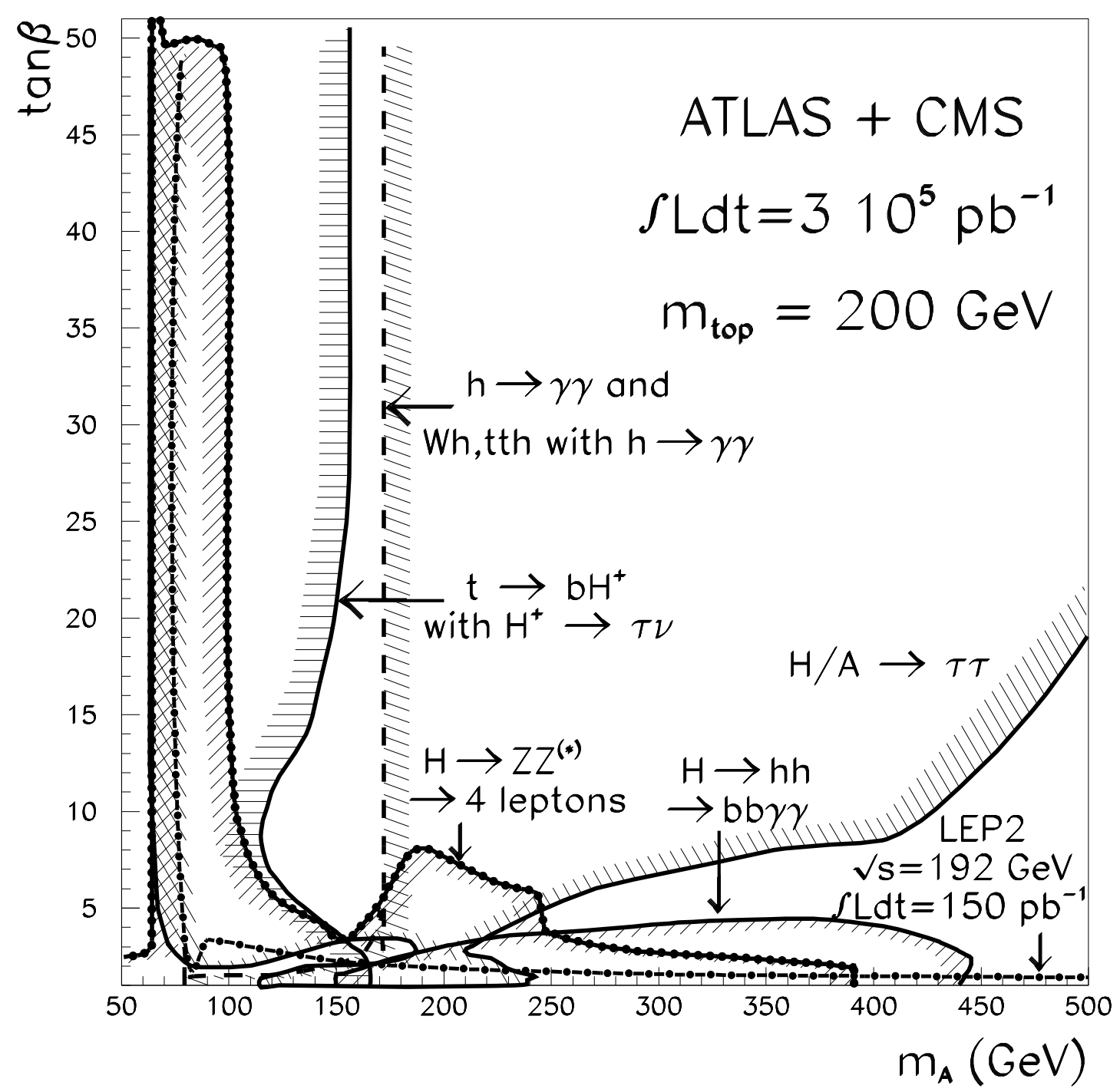

Figure 84: Same as Fig. 82 with a linear scale for $\tan \beta$. 


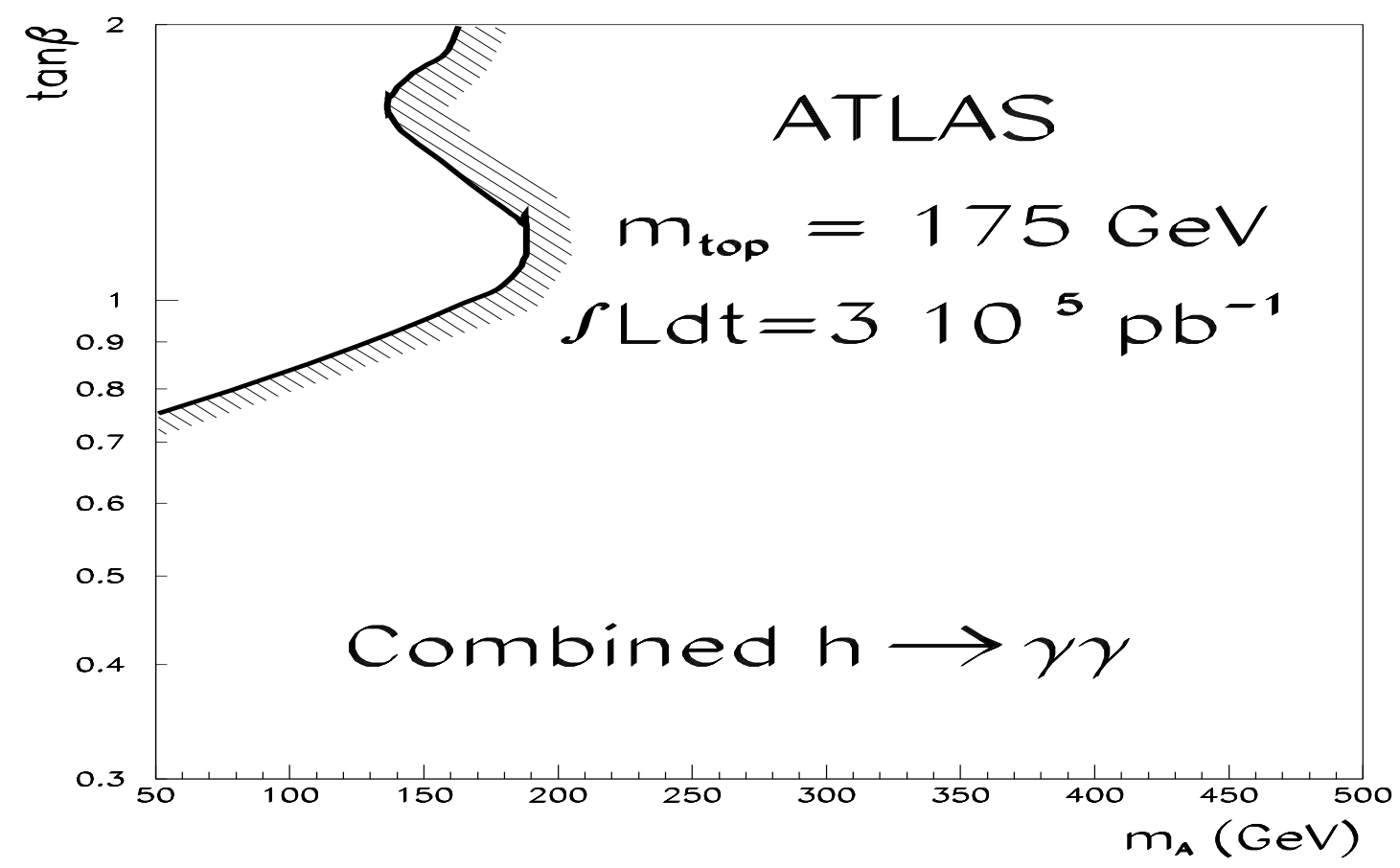

Figure 85: For $m_{\mathrm{t}}=175 \mathrm{GeV}$, an integrated luminosity of $3 \cdot 10^{5} \mathrm{pb}^{-1}$ and $0.3<\tan \beta<2.0,5 \sigma$-discovery contour curve for the combined $\mathrm{h} \rightarrow \gamma \gamma$ inclusive and associated channels in the $\left(m_{\mathrm{A}}, \tan \beta\right)$ plane.

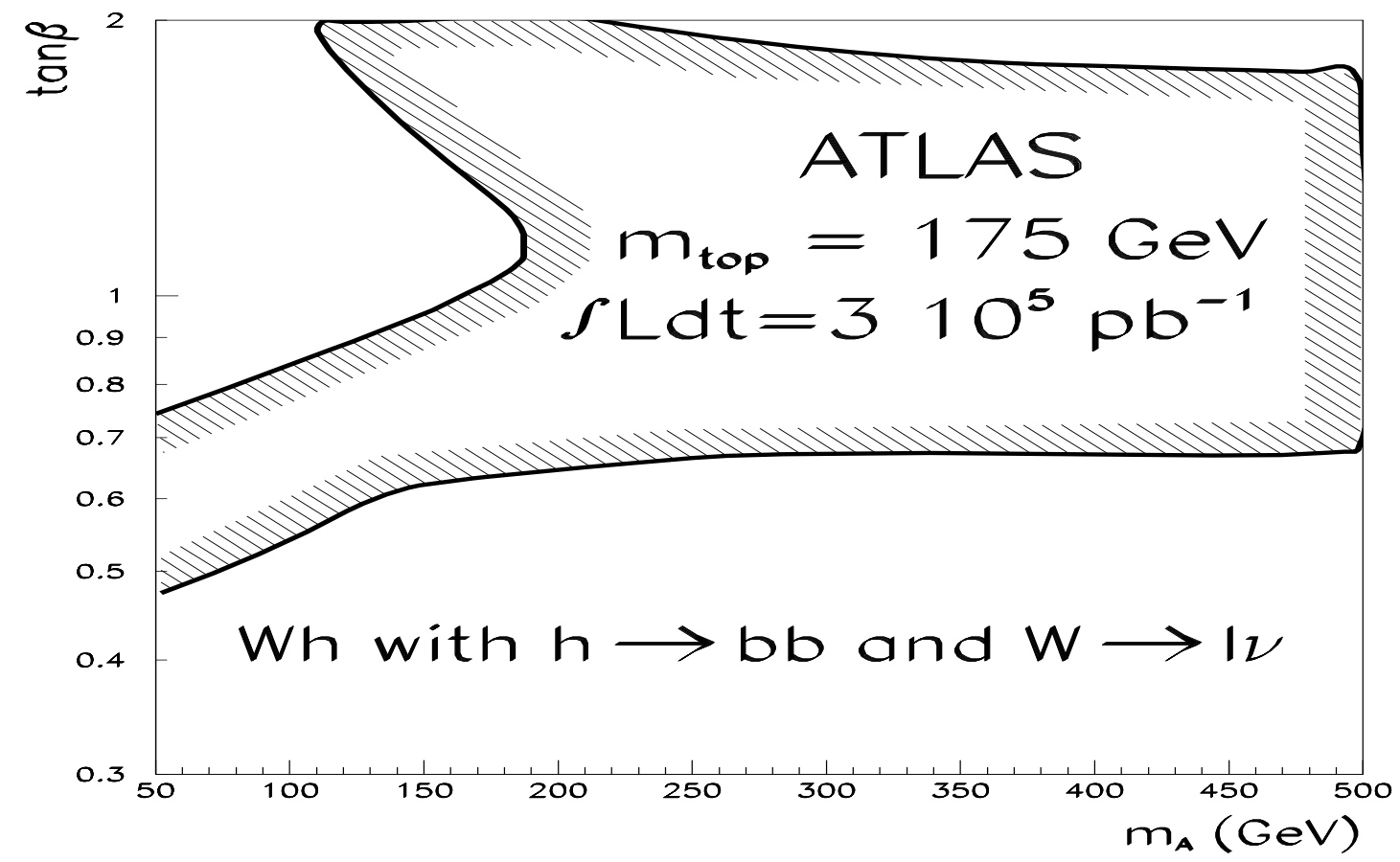

Figure 86: For $m_{\mathrm{t}}=175 \mathrm{GeV}$, an integrated luminosity of $3 \cdot 10^{5} \mathrm{pb}^{-1}$ and $0.3<\tan \beta<2.0,5 \sigma$-discovery contour curve for the $\mathrm{h} \rightarrow \mathrm{b} \overline{\mathrm{b}}$ associated channel in the $\left(m_{\mathrm{A}}, \tan \beta\right)$ plane. 


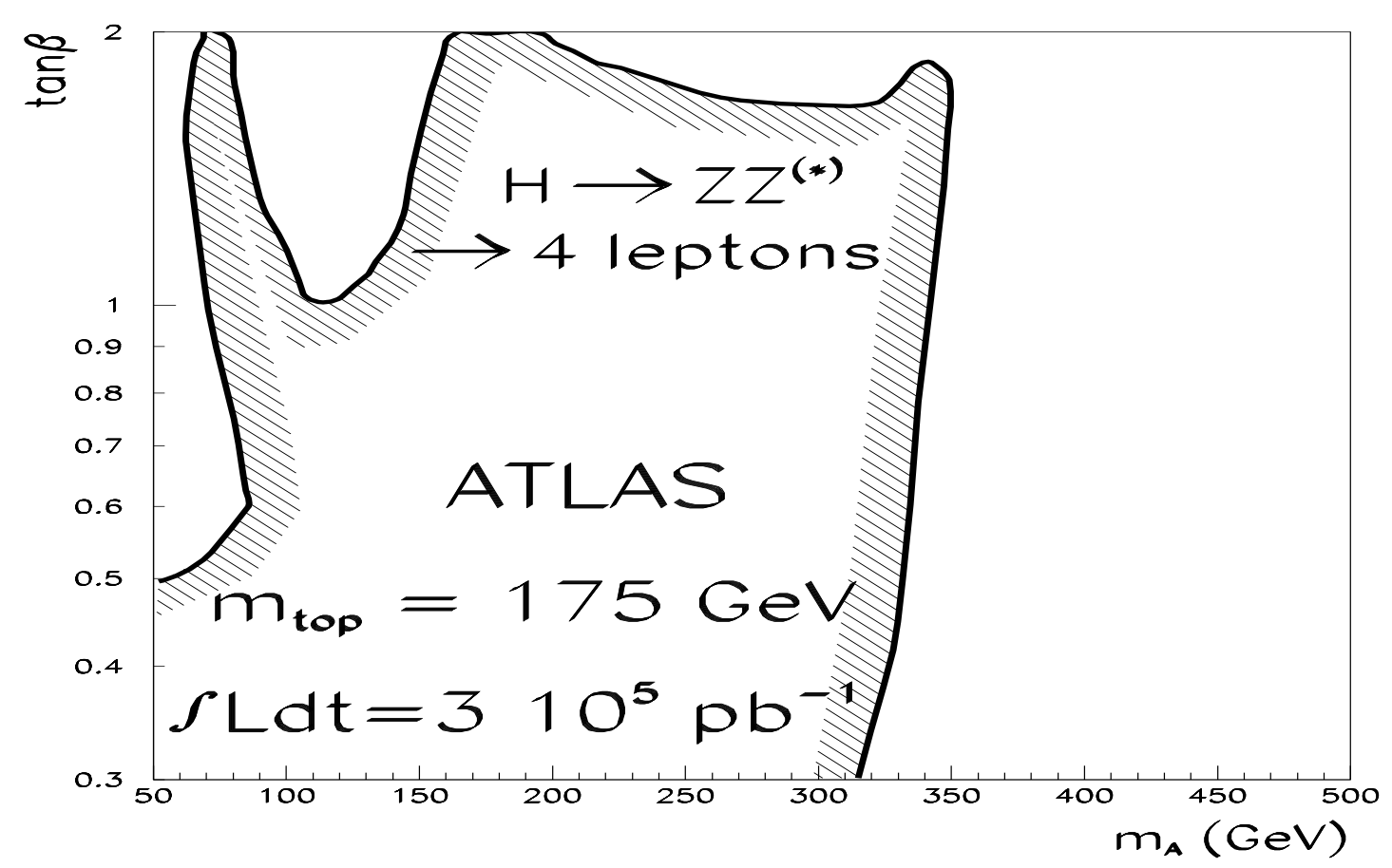

Figure 87: For $m_{\mathrm{t}}=175 \mathrm{GeV}$, an integrated luminosity of $3 \cdot 10^{5} \mathrm{pb}^{-1}$ and $0.3<\tan \beta<2.0,5 \sigma$-discovery contour curve for the $\mathrm{H} \rightarrow \mathrm{ZZ}^{(\star)} \rightarrow 4 \ell$ channel in the $\left(m_{\mathrm{A}}, \tan \beta\right)$ plane.

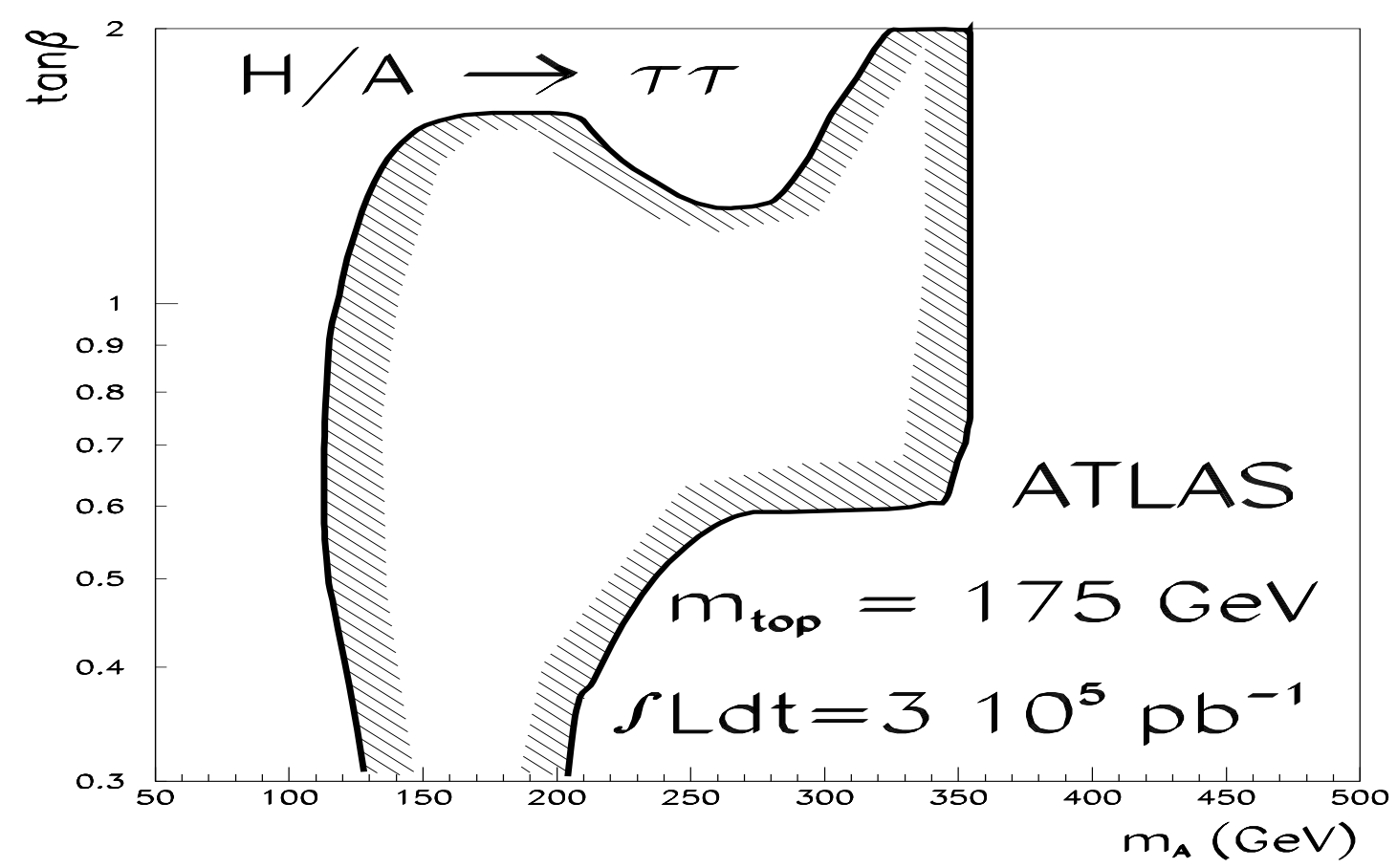

Figure 88: For $m_{\mathrm{t}}=175 \mathrm{GeV}$, an integrated luminosity of $3 \cdot 10^{5} \mathrm{pb}^{-1}$ and $0.3<\tan \beta<2.0,5 \sigma$-discovery contour curve for the combined $\mathrm{H} / \mathrm{A} \rightarrow \tau \tau$ channel in the $\left(m_{\mathrm{A}}, \tan \beta\right)$ plane. 


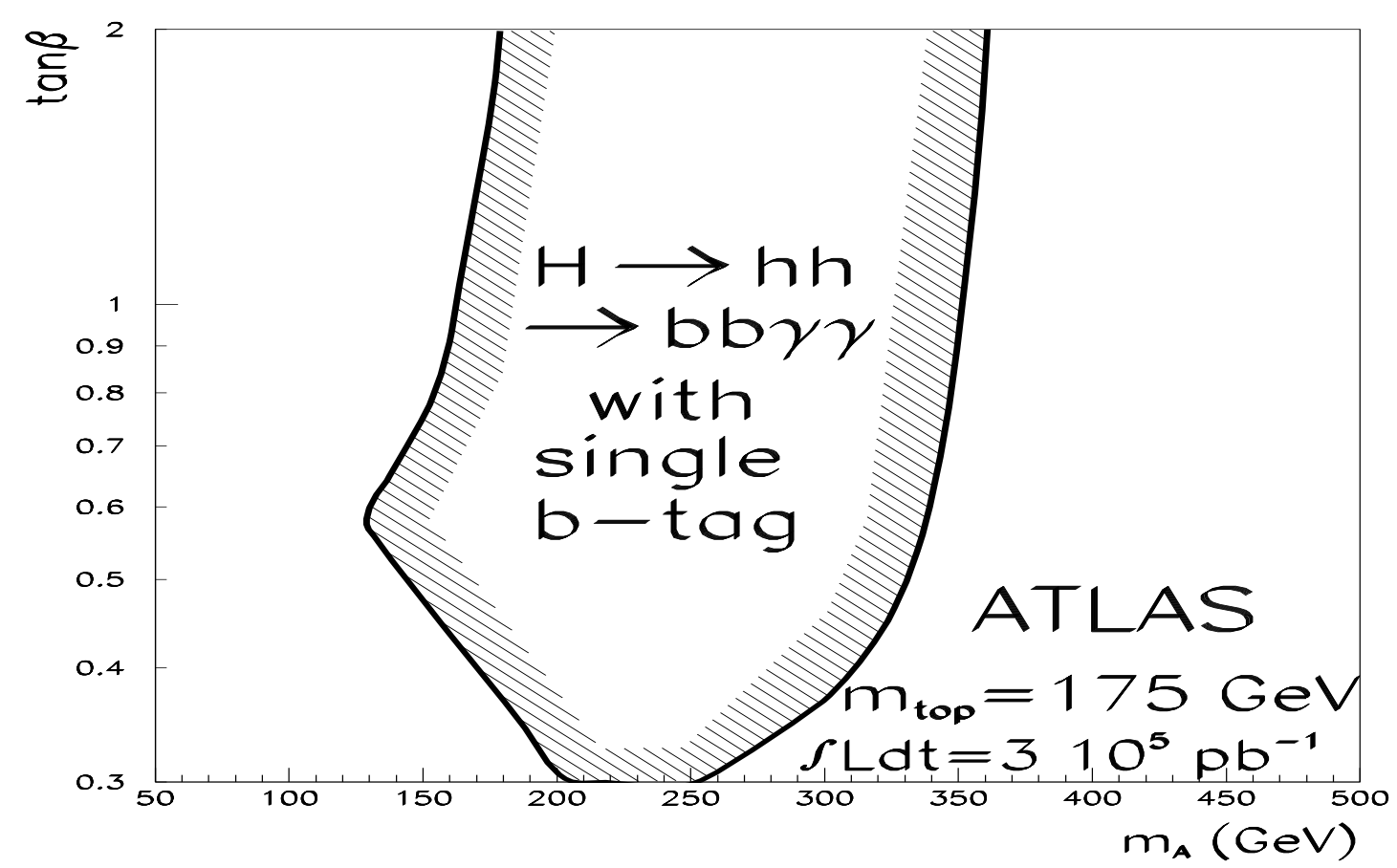

Figure 89: For $m_{\mathrm{t}}=175 \mathrm{GeV}$, an integrated luminosity of $3 \cdot 10^{5} \mathrm{pb}^{-1}$ and $0.3<\tan \beta<2.0,5 \sigma$-discovery contour curve for the $\mathrm{H} \rightarrow \mathrm{hh} \rightarrow \mathrm{b} \overline{\mathrm{b}} \gamma \gamma$ channel in the $\left(m_{\mathrm{A}}, \tan \beta\right)$ plane.

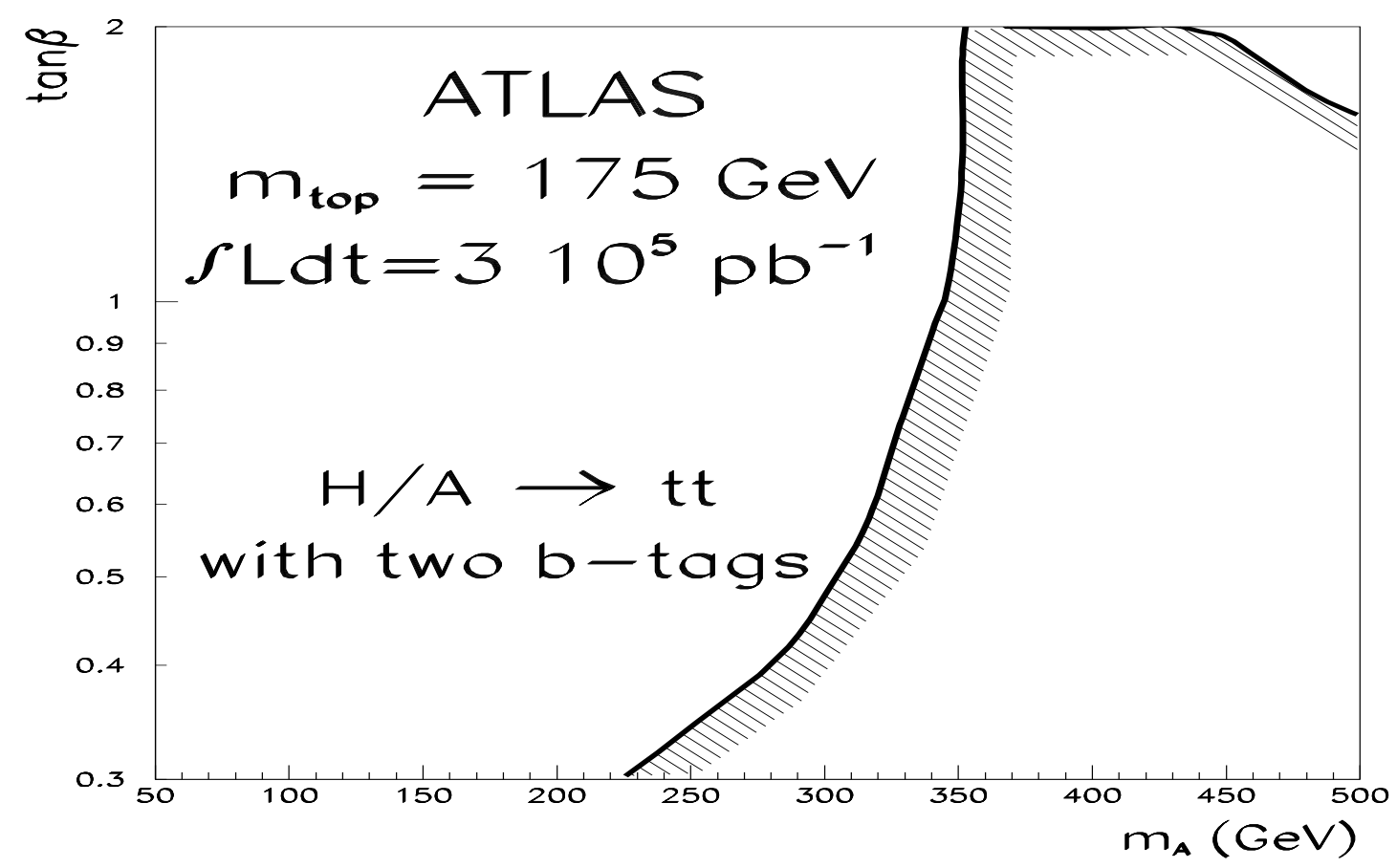

Figure 90: For $m_{\mathrm{t}}=175 \mathrm{GeV}$, an integrated luminosity of $3 \cdot 10^{5} \mathrm{pb}^{-1}$ and $0.3<\tan \beta<2.0,5 \sigma$-discovery contour curve for the combined $\mathrm{H} / \mathrm{A} \rightarrow \mathrm{t} \overline{\mathrm{t}}$ channel in the $\left(m_{\mathrm{A}}, \tan \beta\right)$ plane. 


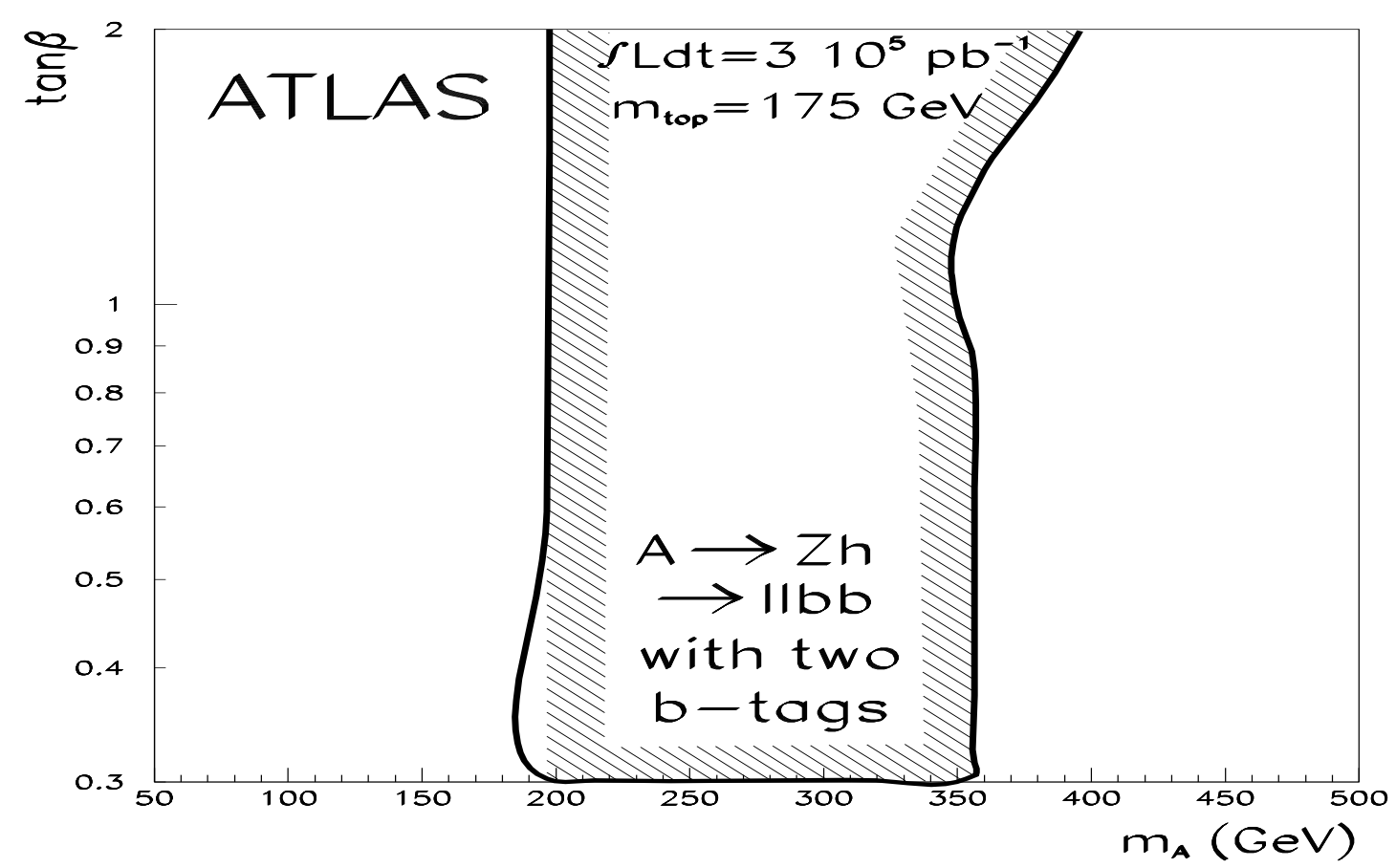

Figure 91: For $m_{\mathrm{t}}=175 \mathrm{GeV}$, an integrated luminosity of $3 \cdot 10^{5} \mathrm{pb}^{-1}$ and $0.3<\tan \beta<2.0,5 \sigma$-discovery contour curves for the $\mathrm{A} \rightarrow \mathrm{Zh} \rightarrow \ell \ell \mathrm{b} \overline{\mathrm{b}}$ channel in the $\left(m_{\mathrm{A}}, \tan \beta\right)$ plane.

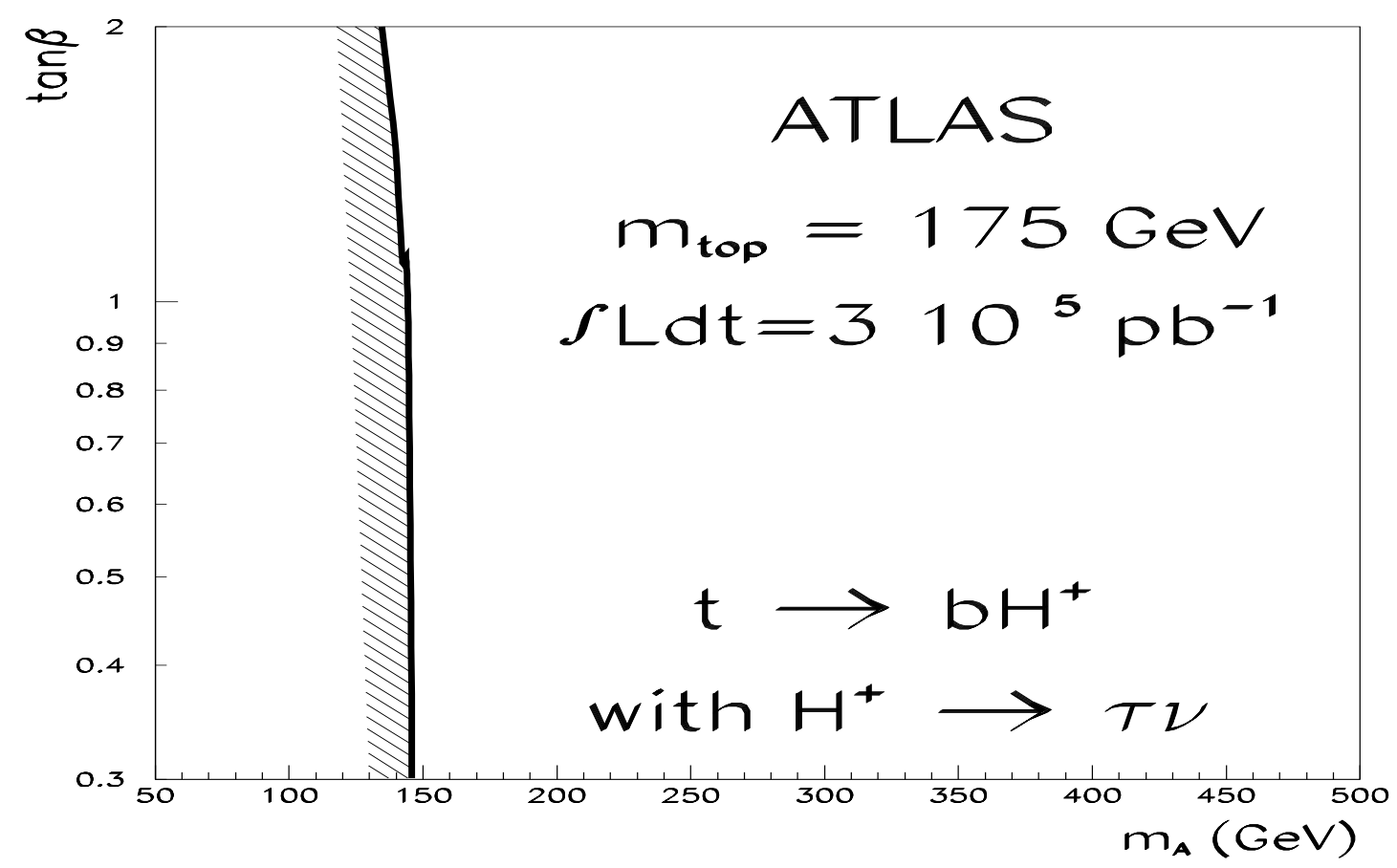

Figure 92: For $m_{\mathrm{t}}=175 \mathrm{GeV}$, an integrated luminosity of $3 \cdot 10^{5} \mathrm{pb}^{-1}$ and $0.3<\tan \beta<2.0,5 \sigma$-discovery contour curves for the $\mathrm{H}^{ \pm} \rightarrow \tau \nu$ channel in the $\left(m_{\mathrm{A}}, \tan \beta\right)$ plane. 
- the overall discovery potential can also be displayed in the $\left(m_{\mathrm{h}}, \tan \beta\right)$ plane, as shown in Fig. 93 for ATLAS with an integrated luminosity of $3 \cdot 10^{5} \mathrm{pb}^{-1}$ and for $m_{\mathrm{t}}=175 \mathrm{GeV}$. This choice of parameter plane, although relevant for LEP2, where most of the sensitivity is related to the h-boson, is not the best one for displaying the LHC potential, because large masses of the other Higgs bosons are all collapsed on top of the line delimiting the maximum allowed value of $m_{\mathrm{h}}$. However, Fig. 93 shows that, for $\tan \beta>5$ and for $82<m_{\mathrm{h}}<95 \mathrm{GeV}$, a discovery of the h-boson through its direct production is impossible at the LHC (see below for a discussion of h-boson discovery through decays of SUSY particles);

- many Higgs boson couplings will be measured at LHC, but with an accuracy not likely to be better than $10-20 \%$, since in most cases these measurements will be based on signal rates. A measurement of obvious interest will be that of the Higgs boson couplings to the top quark, either through the observation of $t \bar{t} h$ production with $h \rightarrow b \bar{b}$ decay, or through the observation of $\mathrm{H} / \mathrm{A} \rightarrow \mathrm{t} \overline{\mathrm{t}}$ decays;

- none of the above conclusions are strongly affected by changes in the model parameters, even if many of the discovery curves change significantly as a function of $m_{\mathrm{t}}$. It is important to recall here that all SUSY particle masses were set to $1 \mathrm{TeV}$ for this study. In some specific cases, the exact choice of the SUSY particle mass spectrum does affect the Higgs boson production cross-sections and/or decay branching ratios, and therefore the discovery potential, as discussed in [8] and in the previous Sections. In particular, preliminary studies based on Minimal Supergravity (SUGRA) Models [28] indicate that the two heavy neutral Higgs bosons and the charged Higgs boson will in many cases have masses larger than $500 \mathrm{GeV}$, i.e. outside the parameter space studied here, and that, for given values of $m_{\mathrm{A}}$ and $\tan \beta$, many different values of $m_{\mathrm{h}}$ are allowed, depending on the exact mass spectrum of SUSY particles.

A better understanding of the observability of the MSSM Higgs sector at the LHC needs more work in some specific channels which involve b-jet tagging, such as $h \rightarrow b \bar{b}$ decays from associated $W h$ and $t \bar{t} h$ production, $\mathrm{H} \rightarrow \mathrm{hh} \rightarrow \mathrm{b} \overline{\mathrm{b}} \mathrm{b} \overline{\mathrm{b}}$ decays, $\mathrm{A} \rightarrow \mathrm{Zh} \rightarrow \mathrm{b} \overline{\mathrm{b}} \mathrm{b} \overline{\mathrm{b}}$ decays, $\mathrm{H} / \mathrm{A} \rightarrow \mathrm{t} \overline{\mathrm{t}}$ decays and possibly bbh, $\mathrm{bbH}$ and bbA production resulting in final states with four bjets. But, more importantly, it needs work to understand the interplay between the SUSY particle sector and the Higgs sector. This work can be done in the context of Minimal SUGRA models, which only have a limited set of parameters.

In conclusion, it is clear that the MSSM Higgs sector is extremely challenging for the LHC experiments and therefore provides an excellent set of benchmark processes to optimise the detector design and performance. This 
is the case for the electromagnetic calorimeter and muon system resolution, for the b-tagging efficiency, for the $\tau$-lepton identification, the $E_{T}^{\text {miss }}$ resolution and also for the hadronic calorimetry in the reconstruction of multijet final states.

The MSSM is however only one model among many and the theoretical predictions based on this model should not be the dominant input into the LHC detector design nor preclude the possibility of investigating other more exotic scenarios. In particular, the Higgs boson signals discussed throughout this study would not provide direct evidence for SUSY, which could only arise from the discovery of supersymmetric particles themselves.

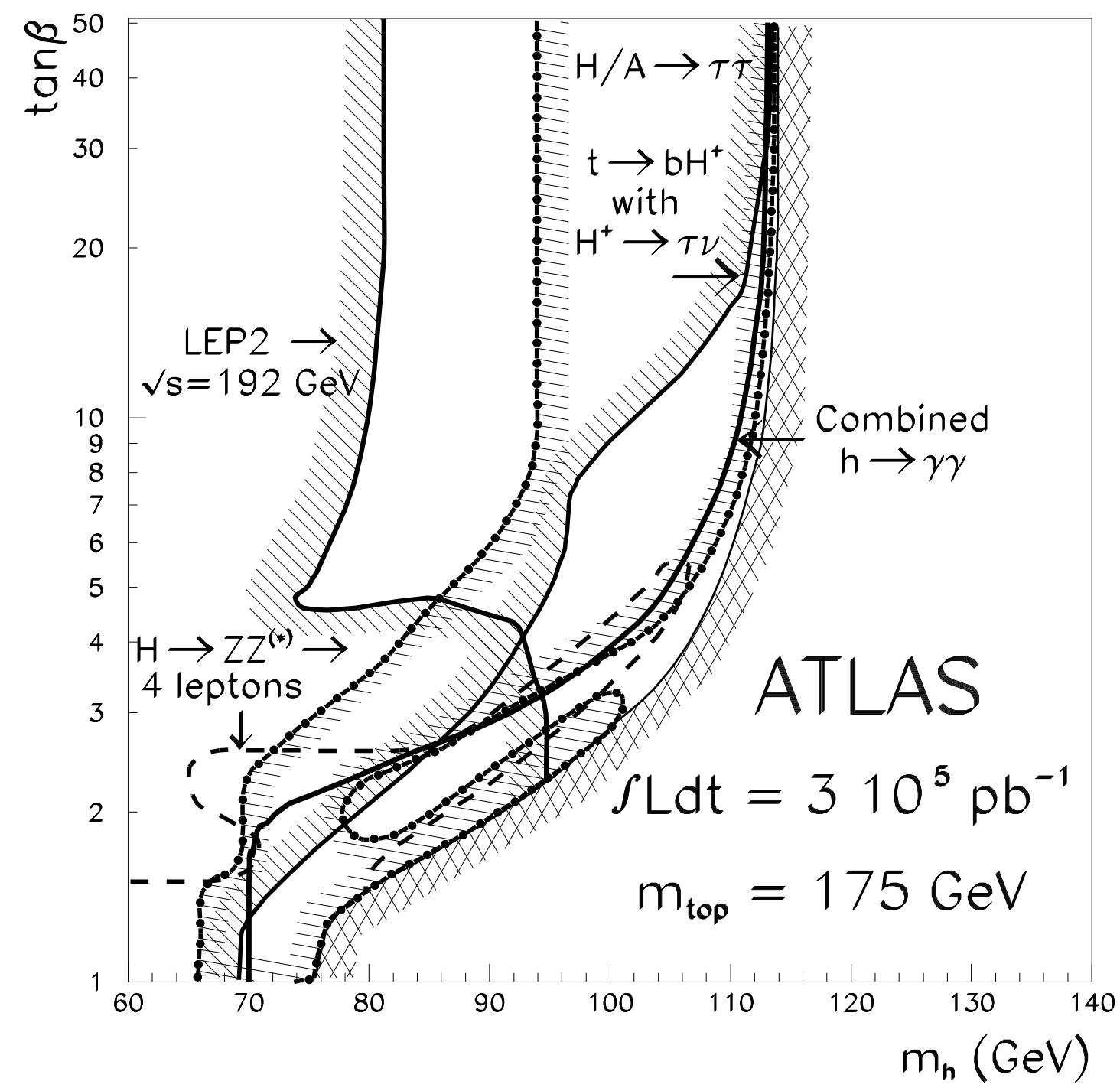

Figure 93: For $m_{\mathrm{t}}=175 \mathrm{GeV}$ and an integrated luminosity of $3 \cdot 10^{5} \mathrm{pb}^{-1}$, ATLAS $5 \sigma$-discovery contour curves in the $\left(m_{\mathrm{h}}, \tan \beta\right)$ plane for all Higgs boson signals discussed in Section 5. The cross-hatched area is theoretically excluded. 


\section{Acknowledgements}

We thank F. Zwirner for providing us with code [6] and for many stimulating and helpful comments, M. Carena and C. Wagner for providing us with code [4], and P. Janot for providing us with the most recent curves for the expected LEP2 discovery limits in the MSSM Higgs sector. We also would like to thank all of our colleagues in ATLAS, without whom this work would not have been possible.

\section{References}

[1] J. F. Gunion, H. E. Haber, G. L. Kane and S. Dawson, The Higgs Hunter's Guide (Addison-Wesley, New York, 1990).

[2] ATLAS Technical Proposal, CERN/LHCC/94-43 (1994).

[3] E. Richter-Was et al., ATLAS Internal Note, PHYS-No-048 (1995); this note and others can be found on WWW at http:://atlasinfo.cern.ch/Atlas/GROUPS/PHYSICS/NOTES

[4] M. Carena et al., Phys. Lett. B355 (1995) 209;

M. Carena et al., CERN preprint CERN-TH/95-45.

[5] A. Brignole et al., Phys. Lett B271 (1991) 123;

J. Ellis, G. Ridolfi and F. Zwirner, Phys. Lett. B257 (1991) 83;

J. Ellis, G. Ridolfi and F. Zwirner, Phys. Lett. B262 (1991) 477.

[6] Z. Kunszt and F. Zwirner, Nucl. Phys. B385 (1992) 3.

[7] F. Abe et al., CDF Collaboration, Phys. Rev. Lett. 73 (1994) 225;

Phys. Rev. D50 (1994) 2966; Phys. Rev. Lett. 74 (1995) 2626;

S. Abachi et al., D0 Collaboration, Phys. Rev. Lett. 74 (1995) 2632.

[8] Interim Report on the Physics Motivations for an Energy Upgrade of LEP2, CERN Preprint CERN-TH/95-151, CERN-PPE/95-78.

[9] A. Djouadi, J. Kalinowski and P.M. Zerwas, Z. Phys. C57 (1993) 569;

A. Djouadi et al., DESY-95-211.

[10] T. Sjöstrand, "High-Energy-Physics Event Generation with PYTHIA 5.7 and JETSET 7.4", CERN preprints CERN-TH.7111/93 and CERNTH.7112/93, Comput. Phys. Commun. 82 (1994) 74.

[11] D. Froidevaux, F. Gianotti and E. Richter-Was, ATLAS Internal Note, PHYS-No-064 (1995).

[12] L. Fayard and G. Unal, EAGLE Internal Note PHYS-No-001 and Addenda 1 and 2 (1992). 
[13] B. Kileng, Z. Phys. C63 (1994) 87;

B. Kileng, P. Osland and N.P. Pandita, NORDITA 95-48-P.

[14] D. Froidevaux and E. Richter-Was, ATLAS Internal Note, PHYS-No043 (1994); Z. Phys. C67 (1995) 213.

[15] I. Gavrilenko et al., ATLAS Internal Note, INDET-No-115 (1995).

[16] L. Poggioli, ATLAS Internal Note, PHYS-No-066 (1995).

[17] O. Linossier and L. Poggioli, ATLAS Internal Note, PHYS-No-075 (1995).

[18] L. Poggioli, D. Froidevaux and C. Guyot, ATLAS Internal Note, PHYSNo-076 (1995).

[19] L. Di Lella, Proceedings of the Large Hadron Collider Workshop (eds. G. Jarlskog and D. Rein), CERN 90-10, Vol. II, p. 530.

[20] D. Cavalli et al., ATLAS Internal Note, PHYS-No-025 (1993);

D. Cavalli et al., ATLAS Internal Note, PHYS-No-051 (1994).

[21] D. Cavalli, ATLAS Internal Note, PHYS-No-080 (1996).

[22] CMS Technical Proposal, CERN/LHCC/94-38 (1994).

[23] J. Bystricki, ATLAS Physics workshop (Trest 1995).

[24] V. Barger, A.L. Stange and R.J.N. Phillips, Phys. Rev. D44 (1991) 1987.

[25] D. Dicus, A. Stange and S. Willenbrock, Phys. Lett. B333 (1994) 126.

[26] B. Paladini, Ph. D. Thesis, University of Milan (1994);

D. Cavalli et al., ATLAS Internal Note, PHYS-No-053 (1994).

[27] UA2 Collaboration, Phys. Lett. B280 (1992) 137.

[28] See e.g. H. Baer et al., Phys. Rev. D52 (1995) 2746.

[29] E. Richter-Was, D. Froidevaux and L. Poggioli, ATLAS Internal Note, PHYS-No-079 (1996).

[30] ATLAS Technical Proposal, see Fig. 3.42, p. 98;

S. Haywood, ATLAS Internal Note, INDET-No-092 and -116;

I. Gavrilenko et al., Atlas Internal Note, INDET-No-115.

[31] F. Gianotti, ATLAS Internal Note, PHYS-No-049;

J. Budagov et al., ATLAS Internal Note, TILE-No-062.

[32] S. D. Ellis, R. Kleiss and W. J. Stirling, Phys. Lett. B154 (1985) 435;

F. A. Berends et al., Phys. Lett. B224 (1989) 530. 


\section{Appendices}

\section{A Particle-level simulation}

Most channels in the MSSM Higgs sector contain a mixture of isolated leptons or photons, $\tau$-leptons, hadronic jets and b-jets in the final state. In many cases, the results presented in this study were obtained from full GEANT simulation and reconstruction of signal and background processes. This was the case wherever particle-level simulations combined with simple parametrisations of the detector response were felt not to be adequate, e.g. for the expected mass resolution in the case of $\mathrm{H} \rightarrow \gamma \gamma, \mathrm{H} \rightarrow \mathrm{ZZ}^{(\star)} \rightarrow 4 \ell$ or $\mathrm{H} / \mathrm{A} \rightarrow \tau \tau$ decays and for the isolated photon $(\mathrm{H} \rightarrow \gamma \gamma$ decays $)$ and $\tau$-lepton $(\mathrm{H} / \mathrm{A} \rightarrow \tau \tau$ decays) reconstruction efficiencies.

In many cases, however, particle-level simulations were used to extract the sensitivity to a specific final state from MSSM Higgs boson production and decay. This procedure was described in detail for the simulation of $H \rightarrow b \bar{b}$ decays in the SM case [14], and was shown to give significantly worse results than parton-level simulations, where quarks and gluons are treated as jets.

After full event generation using PYTHIA 5.707, including initial- and final-state radiation, fragmentation and hadronisation, the final-state stable particles are mapped onto a grid of cells with the granularity of the ATLAS hadronic calorimetry over $|\eta|<5$. Jets are then reconstructed by collecting the energies of all particles (except muons and neutrinos) in a cone around the seed cells with highest energy depositions. Hadronic jets originating from b-quark fragmentation are flagged as b-jets. Photons, electrons and muons are defined as isolated, if well-separated in space from hadronic activity in the event.

The main assumptions and results from this particle-level simulation are summarised below and described in detail in [29].

\section{A.1 Jet reconstruction}

In a first step, all stable particle energies (except those of muons and neutrinos) are stored in cells of granularity $\Delta \eta \times \Delta \phi=0.1 \times 0.1$, with an energy smearing $\sigma_{E}=0.5 \times \sqrt{E} \oplus 0.03 \times E$. Only cells with total transverse energy above a threshold of $1 \mathrm{GeV}$ are used to collect hadronic jet energies in cones of $\Delta R=0.4$. Jets are stored if the transverse energy collected in the cone is $\mathrm{p}_{\mathrm{T}}{ }^{\text {jet }}>15 \mathrm{GeV}$ (at low luminosity) or $\mathrm{p}_{\mathrm{T}}{ }^{\text {jet }}>30 \mathrm{GeV}$ (at high luminosity) and if $\left|\eta^{\text {jet }}\right|<5$.

Jets are then labelled as b-jets, if the jet axis is within the acceptance of the ATLAS Inner Detector, $\left|\eta^{b-j e t}\right|<2.5$ and within $\Delta R<0.2$ of a bquark with $\mathrm{p}_{\mathrm{T}}>5 \mathrm{GeV}$ (b-quarks are considered after inital- and final-state radiation for this matching procedure). 


\section{A.2 Leptons and photons}

Leptons and photons with $\mathrm{p}_{\mathrm{T}}>10 \mathrm{GeV}$ are considered as identifiable if they fall within the acceptance of the combined Inner Detector, electromagnetic calorimetry and muon system, i.e. within $|\eta|<2.5$. In addition, leptons and photons are required to be isolated. At particle-level, the cuts applied to select an isolated lepton or photon are the following:

- the distance $\Delta R=\sqrt{\Delta \eta^{2}+\Delta \phi^{2}}$ between the lepton/photon and any reconstructed jet must be larger than 0.7 ;

- the total transverse energy in a cone, $\Delta R<0.3$, around the lepton/photon is required to be below $10 \mathrm{GeV}$.

These isolation cuts at particle-level cannot be used for any detailed estimate of the reconstruction efficiency for isolated leptons or photons, but only as a rough estimate to be substantiated whenever needed by full GEANT simulation.

Non-isolated leptons and photons are included in the hadronic-jet reconstruction procedure described above. For muons, this procedure is applied only for $\mathrm{p}_{\mathrm{T}}{ }^{\mu}>10 \mathrm{GeV}$ and $\left|\eta^{\mu}\right|<2.5$.

\section{A.3 Parton-level versus particle-level}

The main results obtained from particle-level simulation and used for the study of the MSSM Higgs sector, as well as the main differences observed between parton-level and particle-level simulation, as described in [14], are briefly summarised below:

- the isolation cuts described above result in an acceptance of $\sim 96 \%$ for photons produced through $g g \rightarrow \mathrm{H} \rightarrow \gamma \gamma$. In contrast, bremsstrahlung photons (QED radiation from quarks) are accepted in only $\sim 60 \%$ of the cases for $\mathrm{p}_{\mathrm{T}}>20 \mathrm{GeV}$. This number has been used for the background studies in the $\mathrm{H} \rightarrow \mathrm{hh} \rightarrow \mathrm{b} \overline{\mathrm{b}} \gamma \gamma$ channel;

- the b-jet reconstruction efficiency is only $\sim 77 \%$ for $\mathrm{p}_{\mathrm{T}}{ }^{b-q u a r k}>15 \mathrm{GeV}$. This rather low efficiency is caused by final-state radiation ( $14 \%$ loss) and hadronisation ( $10 \%$ loss) and can be illustrated by the fact that the reconstructed b-jet carries on average only $\sim 80 \%$ of the parent b-quark $\mathrm{p}_{\mathrm{T}}$. Additional losses due to the magnetic field in the Inner Detector are small and were estimated to be $\sim 3 \%$ in [29]. The corresponding efficiency for lightquark jets is $~ 95 \%$;

- if no correction procedure is applied to the reconstructed jet energies, the particle-level study of $\mathrm{H} \rightarrow \mathrm{b} \bar{b}$ decays [14] showed that the reconstructed invariant mass of the two b-jets is shifted to considerably lower mass values, e.g. by $\sim 20 \mathrm{GeV}$ for $m_{\mathrm{H}}=100 \mathrm{GeV}$. In addition, as shown in Fig. 94, the Gaussian part of the mass distribution is significantly wider $\left(\sigma_{m} \sim 11 \mathrm{GeV}\right)$ than for Higgs decays to light quarks $\left(\sigma_{m} \sim 7 \mathrm{GeV}\right)$, and the non-Gaussian tails are very large (more than $20 \%$ of the events lie beyond $\pm 2 \sigma_{m}$ of the mass peak). 

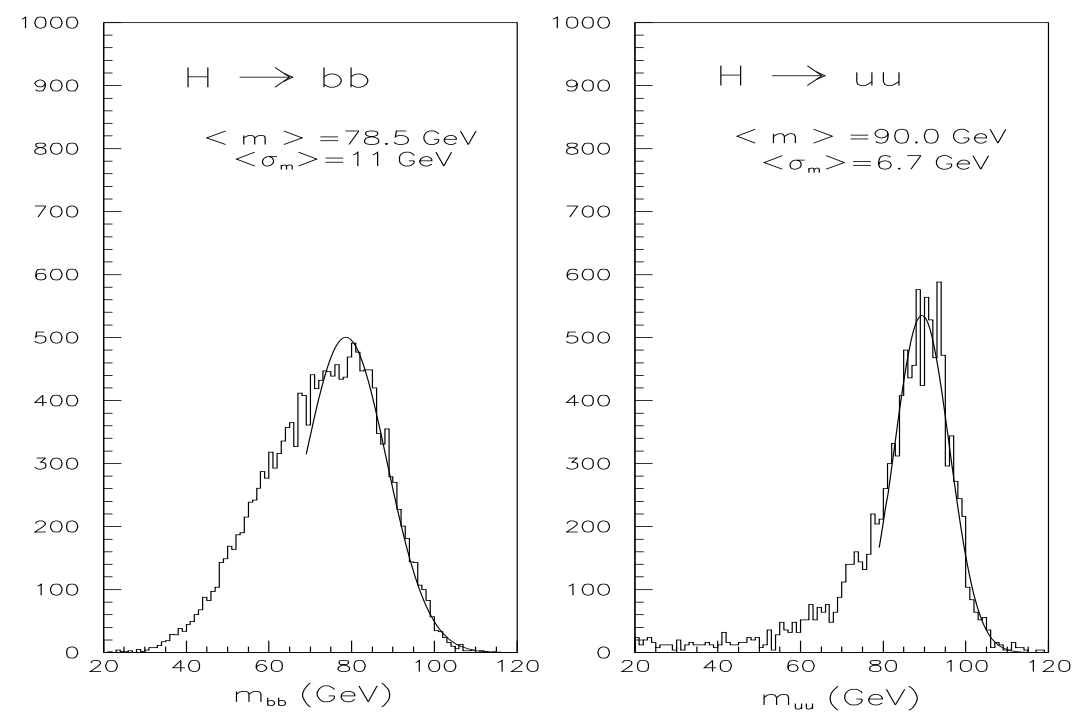

Figure 94: For $m_{\mathrm{H}}=100 \mathrm{GeV}$, reconstructed invariant mass distributions for two b-jets from $\mathrm{H} \rightarrow \mathrm{b} \overline{\mathrm{b}}$ decays and for two light-quark jets from $\mathrm{H} \rightarrow \mathrm{u} \overline{\mathrm{u}}$ decays.

\section{A.4 b-tagging at the LHC}

As discussed in Sections 5 and 6, many final states from Higgs boson decays involve heavy quarks:

- $\mathrm{h} \rightarrow \mathrm{b} \overline{\mathrm{b}}$ from associated Wh and $\mathrm{t} \overline{\mathrm{t}} \mathrm{h}$ production and

- $\mathrm{H} \rightarrow \mathrm{hh} \rightarrow \mathrm{b} \overline{\mathrm{b}} \gamma \gamma$ or $\mathrm{b} \overline{\mathrm{b}} \mathrm{b} \overline{\mathrm{b}}, \mathrm{A} \rightarrow \mathrm{Zh} \rightarrow 1 \mathrm{lb} \overline{\mathrm{b}}$ or $\mathrm{b} \overline{\mathrm{b}} \mathrm{b} \overline{\mathrm{b}}$ and $\mathrm{H} / \mathrm{A} \rightarrow \mathrm{t} \overline{\mathrm{t}}$.

The multiplicity of b-quarks in these final states ranges from two to four, and the backgrounds from processes involving light quark and gluon jets are very large. These very large non-b-jet backgrounds often demand that all b-quarks present in the final state under study be identified. Therefore b-tagging with as high an efficiency as possible, using vertexing algorithms together with soft lepton tags (identified electrons and muons of transverse momenta above 1-2 GeV inside jets), will be one of the keys to a successful search for new physics at the LHC.

As shown experimentally by CDF, and also in a different context by the LEP experiments, vertexing algorithms account for most of the b-tagging performance, while soft lepton tags contribute to a lesser extent, due to the limited fraction of B-hadron decays containing leptons and to the difficulty of efficiently identifying low- $\mathrm{p}_{\mathrm{T}}$ leptons in jets.

Preliminary studies for ATLAS have shown that a b-tagging efficiency of $\sim 50 \%$, with a rejection of $\sim 100$ against non-b-jets, may be reached using vertexing alone at low luminosity [30], i.e. including a vertexing B-layer of high accuracy close to the beam pipe. In addition, b-tagging efficiencies 
of $\sim 10 \%$ per lepton flavour may be achieved with a similar rejection of non-b-jets, using soft lepton tags [31]. Thus, a total b-tagging efficiency $\epsilon_{b}^{\text {tot }}=\epsilon_{b}^{v e r t}+\epsilon_{b}^{e / \mu}-\epsilon_{b}^{v e r t} \epsilon_{b}^{e / \mu}$ of $\sim 60 \%$ may be expected in ATLAS at low luminosity.

At high luminosity, the main degradation with respect to this performance will arise from the absence of the B-layer, unless it can be replaced at regular intervals. The degradation of the performance due to pile-up itself is expected to be small, since the track density in high-p p $_{\mathbf{T}}$ jets is much larger than that due to pile-up events. It is hoped that a total b-tagging efficiency of $\sim 50 \%$ can be achieved at high luminosity with a similar rejection against non-b-jets.

\section{B The $\mathrm{H} / \mathrm{A} \rightarrow \mu \mu$ channel}

This channel, as discussed in Section 5.5, is only accessible at large values of $\tan \beta$. The final state contains two isolated high- $\mathrm{p}_{\mathrm{T}}$ muons, and the signal is observed as a narrow peak in the invariant dimuon mass distribution, $m_{\mu \mu}$. The background is much larger than the signal and consists dominantly of

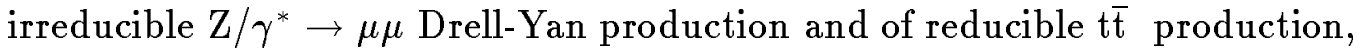
with $\mathrm{t} \rightarrow \mathrm{Wb}$ and $\mathrm{W} \rightarrow \mu \nu$.

A broad range of Higgs masses, from 90 to $500 \mathrm{GeV}$, has been considered for the study presented here. Two isolated muons with $\mathrm{p}_{\mathrm{T}}{ }^{\mu}>20 \mathrm{GeV}$ and $\left|\eta^{\mu}\right|<2.5$, were required in the final state. Several samples of background $\mathrm{Z} / \gamma^{*} \rightarrow \mu \mu$ events were generated for the various mass points by forcing $\sqrt{\hat{s}}$ to be above a given threshold, as shown in Table 40, which gives the $\mathrm{Z} / \gamma^{*} \rightarrow \mu \mu$ background cross-sections as a function of this cut at generation and also the $\mathrm{t} \overline{\mathrm{t}}$ cross-sections, with $\mathrm{t} \rightarrow \mathrm{Wb}$ and $\mathrm{W} \rightarrow \mu \nu$, as a function of $m_{\mathrm{t}}$.

As shown in Fig. 95, the $\mathrm{Z} / \gamma^{*} \rightarrow \mu \mu$ and $\mathrm{t} \overline{\mathrm{t}}$ backgrounds may be reduced somewhat by applying cuts on the dimuon $\mathrm{p}_{\mathrm{T}}, \mathrm{p}_{\mathrm{T}}{ }^{\mu \mu}$, and on $E_{T}^{\mathrm{miss}}$. The cuts chosen were $\mathrm{p}_{\mathrm{T}}{ }^{\mu \mu}>10 \mathrm{GeV}$ for small Higgs masses, and $\mathrm{p}_{\mathrm{T}}{ }^{\mu \mu}>20 \mathrm{GeV}$ for large Higgs masses. The t $\overline{\mathrm{t}}$ background can be reduced by requiring $\mathrm{p}_{\mathrm{T}}{ }^{\mu \mu}<80 \mathrm{GeV}$ for small Higgs masses and more importantly by requiring $E_{T}^{\text {miss }}<30 \mathrm{GeV}$ at low luminosity and $E_{T}^{\text {miss }}<60 \mathrm{GeV}$ at high luminosity. At this stage, the irreducible $\mathrm{Z} / \gamma^{*} \rightarrow \mu \mu$ background is dominant and therefore a jet veto cut would not improve the observability of the signal. The acceptance of these selection cuts for the signal is around 50 to $60 \%$. 

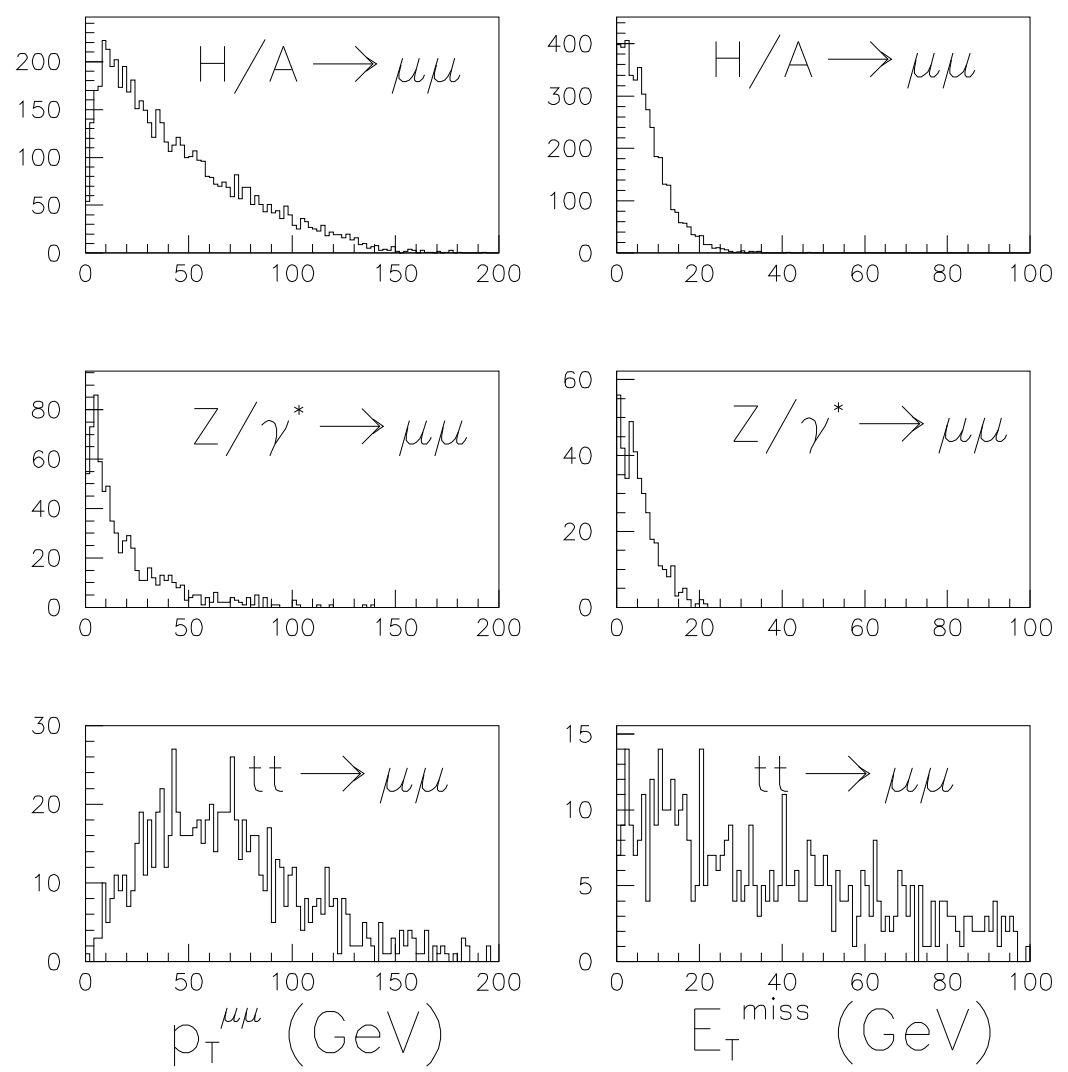

Figure 95: Distributions of $p_{\mathrm{T}}{ }^{\mu \mu}$ and $E_{T}^{\text {miss }}$ in the case of the $H / \mathrm{A} \rightarrow \mu \mu$ signal (top), the $\mathrm{Z} / \gamma^{*} \rightarrow \mu \mu$ background (centre) and the $\mathrm{t} \overline{\mathrm{t}} \rightarrow \mu \mu$ background (bottom), for $m_{\mathrm{H}}, m_{\mathrm{A}}=200 \mathrm{GeV}$ and for events with a dimuon mass in the appropriate mass bin.

The expected signal and background rates at low and high luminosity are shown in Table 41 . These rates were computed, assuming a reconstruction efficiency of $90 \%$ per muon and an acceptance of $90 \%$ for the signal in a mass bin $m_{\mu \mu}=m_{\mathrm{A}} \pm 1.64 \sqrt{\left(\Gamma_{\mathrm{A}}^{\mathrm{tot}} / 2.36\right)^{2}+\sigma_{m}{ }^{2}}$, where $\sigma_{m} \sim 0.02 m_{\mathrm{A}}$. Table 41 also shows the expected observability of the signal as a function of $m_{\mathrm{A}}$ for $\tan \beta=15$. This observability does not depend strongly on $m_{\mathrm{t}}$, as shown in Table 42, and this dependence was therefore neglected for the results shown in Section 5.5. The optimised selection cuts described above do not greatly improve the observability of the signal: this can be seen by comparing the numbers in Tables 41 and 43. The final results, as shown in Table 35 and discussed in Section 5.5, correspond to minimal selection cuts, which only required the presence of two high- $\mathrm{p}_{\mathrm{T}}$ isolated muons with the appropriate invariant mass. 
Table 40: Total cross-sections for the $Z / \gamma^{*} \rightarrow \mu \mu$ background as a function of the generation cuts, and for the $\mathrm{t} \overline{\mathrm{t}} \rightarrow \mu \mu$ background (with $\mathrm{t} \rightarrow \mathrm{Wb}$ and $\mathrm{W} \rightarrow \mu \nu$ ) as a function of $m_{\mathrm{t}}$.

\begin{tabular}{|c||c|c|}
\hline Process & Generation & $\sigma \times B R(\mathrm{pb})$ \\
\hline \hline$Z / \gamma^{*} \rightarrow \mu \mu$ & $\sqrt{\hat{s}}>80 \mathrm{GeV}$ & 1400.0 \\
$Z / \gamma^{*} \rightarrow \mu \mu$ & $\sqrt{\hat{s}}>130 \mathrm{GeV}$ & 12.3 \\
$Z / \gamma^{*} \rightarrow \mu \mu$ & $\sqrt{\hat{s}}>180 \mathrm{GeV}$ & 3.65 \\
$Z / \gamma^{*} \rightarrow \mu \mu$ & $\sqrt{\hat{s}}>280 \mathrm{GeV}$ & 0.81 \\
$Z / \gamma^{*} \rightarrow \mu \mu$ & $\sqrt{\hat{s}}>380 \mathrm{GeV}$ & 0.28 \\
$Z / \gamma^{*} \rightarrow \mu \mu$ & $\sqrt{\hat{s}}>480 \mathrm{GeV}$ & 0.12 \\
\hline $\mathrm{t} \overline{\mathrm{t}} \rightarrow \mu \mu$ & $m_{\mathrm{t}}=150 \mathrm{GeV}$ & 13.6 \\
$\mathrm{t} \overline{\mathrm{t}} \rightarrow \mu \mu$ & $m_{\mathrm{t}}=175 \mathrm{GeV}$ & 7.0 \\
$\mathrm{t} \overline{\mathrm{t}} \rightarrow \mu \mu$ & $m_{\mathrm{t}}=200 \mathrm{GeV}$ & 3.9 \\
\hline
\end{tabular}

Table 41: Observability of the $H / \mathrm{A} \rightarrow \mu \mu$ channel for $m_{\mathrm{t}}=175 \mathrm{GeV}$ and for integrated luminosities of $3 \cdot 10^{4} \mathrm{pb}^{-1}$ (top) and $10^{5} \mathrm{pb}^{-1}$ (bottom). The $\sigma \times B R$ values and the expected numbers of signal and background events are given for $\mathrm{A} \rightarrow \mu \mu$ decays and for $\tan \beta=15$. The $p_{\mathrm{T}}{ }^{\mu \mu}$ and $E_{T}^{\text {miss }}$ cuts described in the text are applied.

\begin{tabular}{|c||c||c|c|c||c|}
\hline$m_{\mathrm{A}}(\mathrm{GeV})$ & $\sigma \times B R(\mathrm{fb})$ & Signal & $Z / \gamma^{*} \rightarrow \mu \mu$ & $t \bar{t}$ & $\mathrm{~S} / \sqrt{\mathrm{B}}$ \\
\hline \hline 90 & 106 & 1040 & $5.0 \cdot 10^{6}$ & 1340 & 0.46 \\
\hline 100 & 76.7 & 838 & $\mathbf{0 . 7} \cdot 10^{6}$ & 1260 & 0.84 \\
\hline 120 & 36.6 & 370 & 51030 & 1007 & 1.62 \\
\hline 150 & 14.2 & 145 & 12070 & 1090 & 1.26 \\
\hline 200 & 5.32 & 71 & 5240 & 1045 & 0.90 \\
\hline 300 & 1.12 & 15 & 1100 & 390 & 0.40 \\
\hline 400 & 0.36 & 5 & 506 & 98 & 0.20 \\
\hline 500 & 0.13 & 6 & 238 & 60 & 0.35 \\
\hline
\end{tabular}

\begin{tabular}{|c||c||c|c|c||c|}
\hline$m_{\mathrm{A}}(\mathrm{GeV})$ & $\sigma \times B R(\mathrm{fb})$ & Signal & $Z / \gamma^{*} \rightarrow \mu \mu$ & $t \bar{t}$ & $\mathrm{~S} / \sqrt{\mathrm{B}}$ \\
\hline \hline 90 & 106 & 3360 & $16.7 \cdot 10^{6}$ & 7730 & 0.8 \\
\hline 100 & 76.7 & 2531 & $2.3 \cdot 10^{6}$ & 7475 & 1.7 \\
\hline 120 & 36.6 & 1246 & 170100 & 5622 & 3.0 \\
\hline 150 & 14.2 & 480 & 40250 & 5790 & 2.2 \\
\hline 200 & 5.32 & 236 & 17470 & 5790 & 1.5 \\
\hline 300 & 1.12 & 49 & 3670 & 2090 & 0.6 \\
\hline 400 & 0.36 & 17 & 1700 & 750 & 0.3 \\
\hline 500 & 0.13 & 6 & 790 & 340 & 0.2 \\
\hline
\end{tabular}


Table 42: Observability of the $H / \mathrm{A} \rightarrow \mu \mu$ channel for an integrated luminosity of $10^{5} \mathrm{pb}^{-1}$ and as a function of $m_{\mathrm{t}}$. The $\sigma \times B R$ values and the expected numbers of signal and background events are given for $\mathrm{A} \rightarrow \mu \mu$ decays and for $\tan \beta=15$. The $p_{\mathrm{T}}{ }^{\mu \mu}$ and $E_{T}^{\text {miss }}$ cuts described in the text are applied.

\begin{tabular}{|c||c||c|c|c||c|}
\hline$m_{\mathrm{A}}(\mathrm{GeV})$ & $\begin{array}{c}\sigma \times B R \\
(\mathrm{fb})\end{array}$ & Signal & $\begin{array}{c}\mathrm{S} / \sqrt{\mathrm{B}} \\
m_{\mathrm{t}}=150 \mathrm{GeV}\end{array}$ & $\begin{array}{c}\mathrm{S} / \sqrt{\mathrm{B}} \\
m_{\mathrm{t}}=175 \mathrm{GeV}\end{array}$ & $\begin{array}{c}\mathrm{S} / \sqrt{\mathrm{B}} \\
m_{\mathrm{t}}=200 \mathrm{GeV}\end{array}$ \\
\hline \hline 90 & 106 & 3360 & $\mathbf{0 . 8}$ & $\mathbf{0 . 8}$ & $\mathbf{0 . 8}$ \\
\hline 100 & 76.7 & 2531 & 1.7 & 1.7 & 1.7 \\
\hline 120 & 36.6 & 1246 & 2.9 & 3.0 & 3.0 \\
\hline 150 & 14.2 & 480 & 2.1 & 2.2 & 2.3 \\
\hline 200 & 5.32 & 236 & 1.4 & 1.5 & 1.6 \\
\hline 300 & 1.12 & 49 & $\mathbf{0 . 6}$ & $\mathbf{0 . 6}$ & $\mathbf{0 . 7}$ \\
\hline 400 & $\mathbf{0 . 3 6}$ & 17 & $\mathbf{0 . 3}$ & $\mathbf{0 . 3}$ & $\mathbf{0 . 3}$ \\
\hline 500 & $\mathbf{0 . 1 3}$ & 6 & $\mathbf{0 . 2}$ & $\mathbf{0 . 2}$ & $\mathbf{0 . 2}$ \\
\hline \hline
\end{tabular}

Table 43: Observability of the $H / \mathrm{A} \rightarrow \mu \mu$ channel for an integrated luminosity of $10^{5} \mathrm{pb}^{-1}$ and for $m_{\mathrm{t}}=175 \mathrm{GeV}$. The $\sigma \times B R$ values and the expected numbers of signal and background events are given for $\mathrm{A} \rightarrow \mu \mu$ decays and for $\tan \beta=15$. The $p_{\mathrm{T}}{ }^{\mu \mu}$ and $E_{T}^{\text {miss }}$ cuts described in the text are not applied.

\begin{tabular}{|c||c||c|c|c||c|}
\hline$m_{\mathrm{A}}(\mathrm{GeV})$ & $\sigma \times B R(\mathrm{fb})$ & Signal & $Z / \gamma^{*} \rightarrow \mu \mu$ & $t \bar{t}$ & $\mathrm{~S} / \sqrt{\mathrm{B}}$ \\
\hline \hline 90 & 106 & 4338 & $35.3 \cdot 10^{6}$ & 15300 & 0.7 \\
\hline 100 & 76.7 & 3268 & $4.8 \cdot 10^{6}$ & 14670 & 1.5 \\
\hline 120 & 36.6 & 1640 & 260800 & 11640 & 3.1 \\
\hline 150 & 14.2 & 672 & 74020 & 11950 & 2.3 \\
\hline 200 & 5.32 & 260 & 27670 & 8200 & 1.4 \\
\hline 300 & 1.12 & 58 & 7750 & 2760 & 0.6 \\
\hline 400 & 0.36 & 20 & 3070 & 1000 & 0.3 \\
\hline 500 & 0.13 & 7 & 1380 & 480 & 0.2 \\
\hline
\end{tabular}

\section{The $\mathrm{H} \rightarrow \mathrm{hh} \rightarrow \mathrm{b} \overline{\mathrm{b}} \gamma \gamma$ channel}

As discussed in Section 5.6, the observation of this channel would be particularly interesting, since it would correspond to the simultaneous discovery of two Higgs bosons. The $\mathrm{H} \rightarrow \mathrm{hh} \rightarrow \mathrm{b} \overline{\mathrm{b}} \gamma \gamma$ channel was the only one studied here, because it can be easily triggered upon and it offers good kinematic constraints for the reconstruction of $m_{\mathrm{H}}$. In particular, the study was extended to values of $m_{\mathrm{h}}$ as low as $60 \mathrm{GeV}$. The final state consists of two high-p $\mathrm{p}_{\mathrm{T}}$ isolated photons and two b-jets. Since the signal rate is low, only one of the b-jets is required to be tagged by the detector. 
Several background sources were considered: irreducible $\mathrm{b} \overline{\mathrm{b}} \gamma \gamma$ and reducible $\operatorname{bj} \gamma \gamma, \operatorname{cc} \gamma \gamma, \operatorname{cj} \gamma \gamma$ and $\mathrm{jj} \gamma \gamma$. Large uncertainties apply to these background estimates, due to the poor knowledge of the total $b \bar{b}, c \bar{c}, q \gamma$ and jj cross-sections, and to the procedure used to simulate photon bremsstrahlung in these processes. This procedure used the parton-shower approximation for generating QED bremsstrahlung, as provided by PYTHIA 5.707, and turned out to be very CPU time-consuming. The optimisation of the selection criteria to extract the signal from the background had therefore to be performed with limited statistics.

Table 44 shows, for each of the background channels, the production crosssections, the cuts applied at event generation, the numbers of events generated $\left(N_{\text {gener }}\right)$, and the numbers of events selected $\left(N_{\text {select }}\right)$ with two high-p $\mathrm{p}_{\mathrm{T}}$ isolated photons and two jets within the acceptance of the detector. To estimate these backgrounds as correctly as possible, the cross-sections for the $\mathrm{b} \overline{\mathrm{b}}$ and the $c \overline{\mathrm{c}}$ backgrounds were multiplied by factors of 1.7 and 1.3 respectively, to account for the production of $b \bar{b}$ and $c \bar{c}$ pairs in the $q \gamma$ process. These factors were obtained by comparing, within limited statistics, the rates of $\mathrm{b} \bar{b} \gamma \gamma$ and $c \bar{c} \gamma \gamma$ events, arising from $b \bar{b}, c \bar{c}$ and $q \gamma$ processes.

Table 44: For the dominant background processes to the $\mathrm{H} \rightarrow \mathrm{hh} \rightarrow \mathrm{b} \overline{\mathrm{b}} \gamma \gamma$ channel, production cross-sections, cuts applied at event generation, numbers of events generated ( $\left.N_{\text {gener }}\right)$, and numbers of events selected $\left(N_{\text {select }}\right)$, as described in text.

\begin{tabular}{|c||c|c|c|c|}
\hline Process & Cuts at generation & $\sigma(\mathrm{pb})$ & $N_{\text {gener }}$ & $N_{\text {select }}$ \\
\hline \hline $\mathrm{b} \overline{\mathrm{b}}$ & $\sqrt{\hat{s}}>180 \mathrm{GeV}$ & $1.3 \cdot 10^{5} \mathrm{pb}$ & $3.0 \cdot 10^{9}$ & 1491 \\
\hline $\mathrm{c} \overline{\mathrm{c}}$ & $\sqrt{\hat{s}}>180 \mathrm{GeV}$ & $2.3 \cdot 10^{5} \mathrm{pb}$ & $1.0 \cdot 10^{9}$ & 4679 \\
\hline $\mathrm{q} \gamma$ & $\begin{array}{c}\sqrt{\hat{s}}>70 \mathrm{GeV} \\
\mathrm{p}_{\mathrm{T}}^{\text {hard }}>10 \mathrm{GeV}\end{array}$ & $4.8 \cdot 10^{4} \mathrm{pb}$ & $1.6 \cdot 10^{8}$ & 30675 \\
\hline
\end{tabular}

The signal and background events were analysed at particle-level with the photons and b-jets reconstructed as described in Appendix A.1. The signal was extracted by requiring two isolated photons, with $|\eta|<2.5$ and $\mathrm{p}_{\mathrm{T}}>20 \mathrm{GeV}$, and two additional jets with $|\eta|<2.5$ and $\mathrm{p}_{\mathrm{T}}>15 \mathrm{GeV}$ (resp. $\mathrm{p}_{\mathrm{T}}>30 \mathrm{GeV}$ ) at low (resp. high) luminosity. The signal acceptance depends somewhat on $m_{\mathrm{H}}$ and $m_{\mathrm{h}}$, and is $\sim 20 \%$ at low luminosity and $\sim 10 \%$ at high luminosity. At least one of the jets was required to be tagged as a b-jet with an assumed efficiency $\epsilon_{b}=60 \%$ (resp. 50\%) at low (resp. high) luminosity, corresponding to a rejection of $\sim 100$ against non-b-jets and $\sim 10$ against c-jets. The photon reconstruction efficiency was assumed to be $80 \%$ per photon as in the $\mathrm{H} \rightarrow \gamma \gamma$ case.

Events were accepted if the diphoton mass was within $\pm 2 \mathrm{GeV}$ of $m_{\mathrm{h}}$, and if the dijet mass was within $\pm 20 \mathrm{GeV}$ of $m_{\mathrm{h}}-20 \mathrm{GeV}$ (no correction 
to the reconstructed dijet mass was applied in this study). Figure 96 shows the reconstructed $\mathrm{m}_{\gamma \gamma}$ and $m_{\mathrm{b} \overline{\mathrm{b}}}$ mass distributions for $m_{\mathrm{H}}=320 \mathrm{GeV}$ and $m_{\mathrm{h}}=70 \mathrm{GeV}$. The low-energy tail discussed in Appendix A.3 is clearly visible in the $m_{\mathrm{b} \overline{\mathrm{b}}}$ distribution. Figure 96 also shows the reconstructed $m_{\mathrm{b} \overline{\mathrm{b}} \gamma \gamma}$ distribution before and after rescaling the photon and jet 4-momenta appropriately by applying a constraint on $m_{\mathrm{h}}$. This constraint improves the overall mass resolution from $\sim 14 \mathrm{GeV}$ to $\sim 7 \mathrm{GeV}$ and reduces significantly the fraction of non-Gaussian tails. The signal acceptance in a mass bin of $\pm 10 \mathrm{GeV}$ around $m_{\mathrm{H}}$ is $\sim 90 \%$ after the $m_{\mathrm{h}}$ mass constraint.
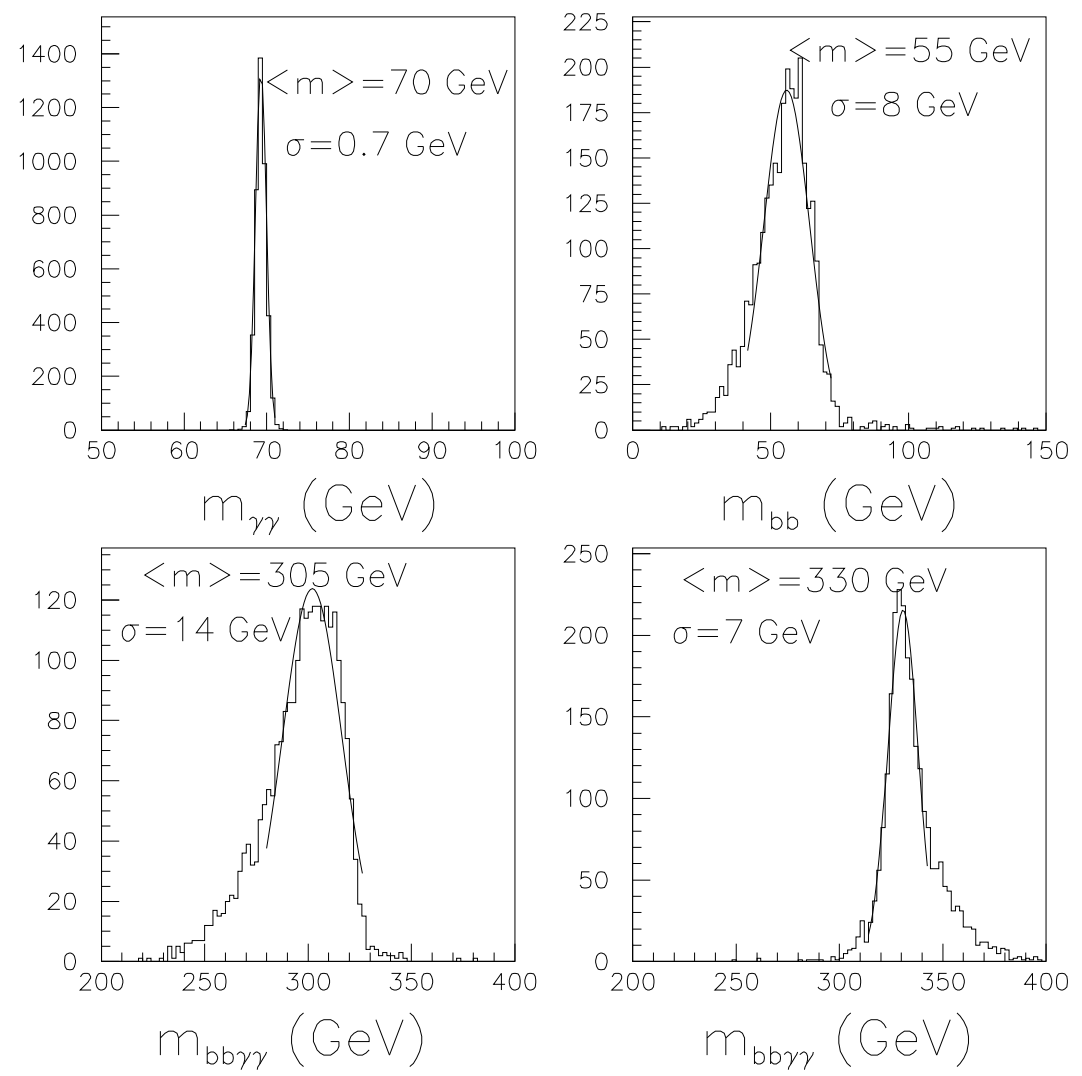

Figure 96: For $\mathrm{H} \rightarrow \mathrm{hh} \rightarrow \mathrm{b} \overline{\mathrm{b}} \gamma \gamma$ decays with $m_{\mathrm{H}}=320$ GeV and $m_{\mathrm{h}}=70 \mathrm{GeV}$, reconstructed mass distributions for $m_{\gamma \gamma}, m_{\mathrm{b} \overline{\mathrm{b}}}$ and $m_{\mathrm{b} \overline{\mathrm{b}} \gamma \gamma}$ before and after using the $m_{\mathrm{h}}$ mass constraint.

The signal and background rates were estimated for eight mass points, corresponding to $m_{\mathrm{H}}=231,275,321$ and $368 \mathrm{GeV}$ and to $m_{\mathrm{h}} \sim 72 \mathrm{GeV}$ $(\tan \beta=1)$ and to $m_{\mathrm{h}} \sim 97 \mathrm{GeV}(\tan \beta=3)$, and for both the low- and high-luminosity selection cuts described above. Due to the limited statistics in the background samples, the background contributions were estimated by using larger mass bins than the ones chosen to extract the signal, i.e. the 
$\mathrm{m}_{\gamma \gamma}$ mass bin was increased from $\pm 2 \mathrm{GeV}$ to $\pm 30 \mathrm{GeV}$, the $m_{\mathrm{b} \overline{\mathrm{b}}}$ mass bin from $\pm 20 \mathrm{GeV}$ to $\pm 40 \mathrm{GeV}$ and the $m_{\mathrm{b} \overline{\mathrm{b}} \gamma \gamma}$ mass bin from $\pm 10 \mathrm{GeV}$ to $\pm 30 \mathrm{GeV}$. In the cases where no background event was left after cuts, an upper limit of one background event was used.

The various photon bremsstrahlung backgrounds described above were further reduced by requiring that the larger of the two h-boson transverse momenta, $\max \left(\mathrm{p}_{\mathrm{T}}{ }^{b b}, \mathrm{p}_{\mathrm{T}}{ }^{\gamma \gamma}\right)$, be larger than 60 to $80 \mathrm{GeV}$, as shown in Fig. 97, and that the invariant $\gamma \gamma j$ masses, $\max \left(\mathrm{m}_{\gamma \gamma j 1}, \mathrm{~m}_{\gamma \gamma j 2}\right)$ and $\min \left(\mathrm{m}_{\gamma \gamma j 1}, \mathrm{~m}_{\gamma \gamma j 2}\right)$, be larger than 150 to $200 \mathrm{GeV}$ and 100 to $150 \mathrm{GeV}$ respectively, as shown in Fig. 98. These cuts were optimised for each value of $m_{\mathrm{H}}$ and $m_{\mathrm{h}}$.
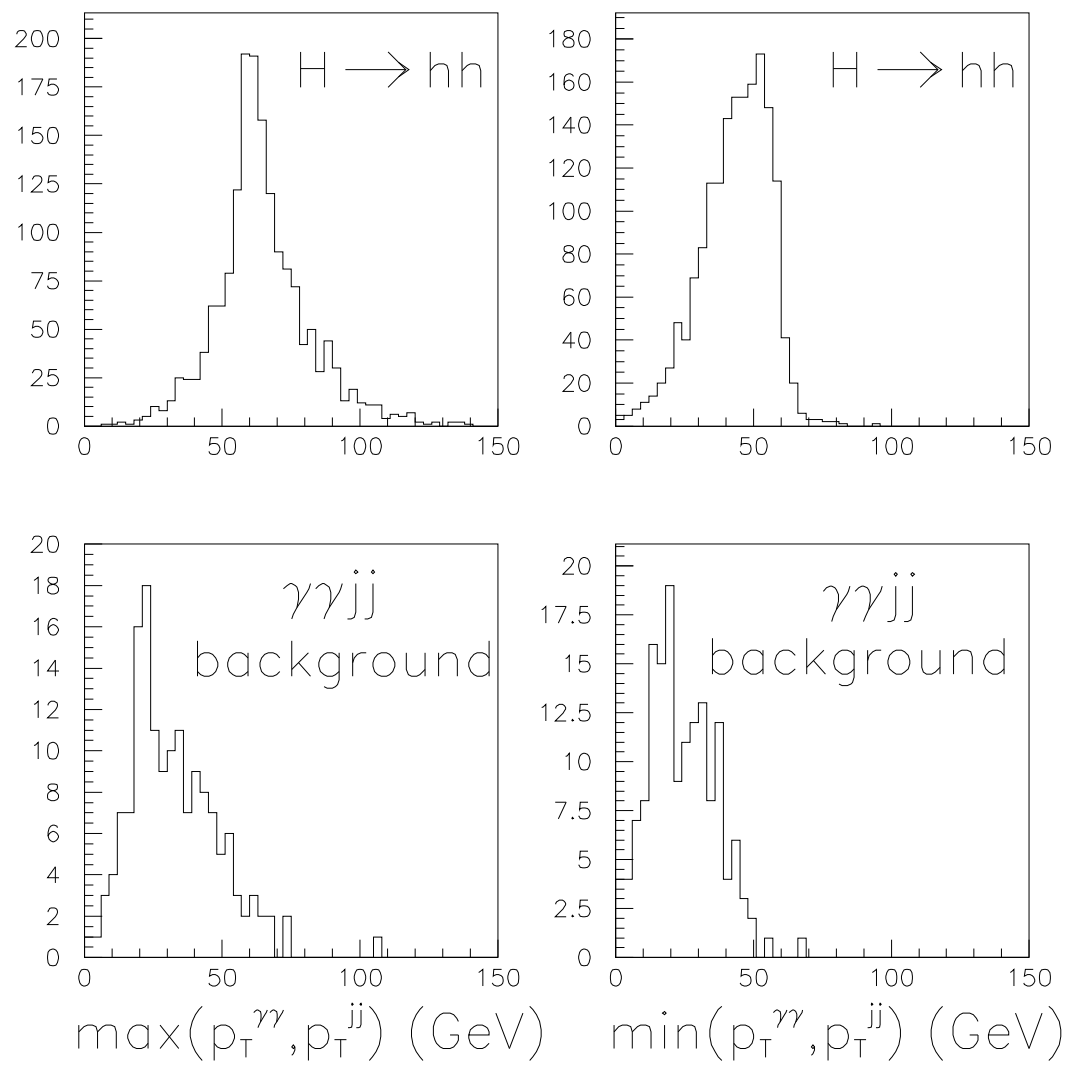

Figure 97: For $\mathrm{H} \rightarrow \mathrm{hh} \rightarrow \mathrm{b} \overline{\mathrm{b}} \gamma \gamma$ decays with $m_{\mathrm{H}}=321$ GeV and $m_{\mathrm{h}}=70 \mathrm{GeV}$ and for the residual $\gamma \gamma j j$ background, distributions of $\max \left(p_{\mathrm{T}}{ }^{b b}, p_{\mathrm{T}}{ }^{\gamma \gamma}\right)$ and of $\min \left(p_{\mathrm{T}}^{b b}, p_{\mathrm{T}}^{\gamma \gamma}\right)$ (see text). 

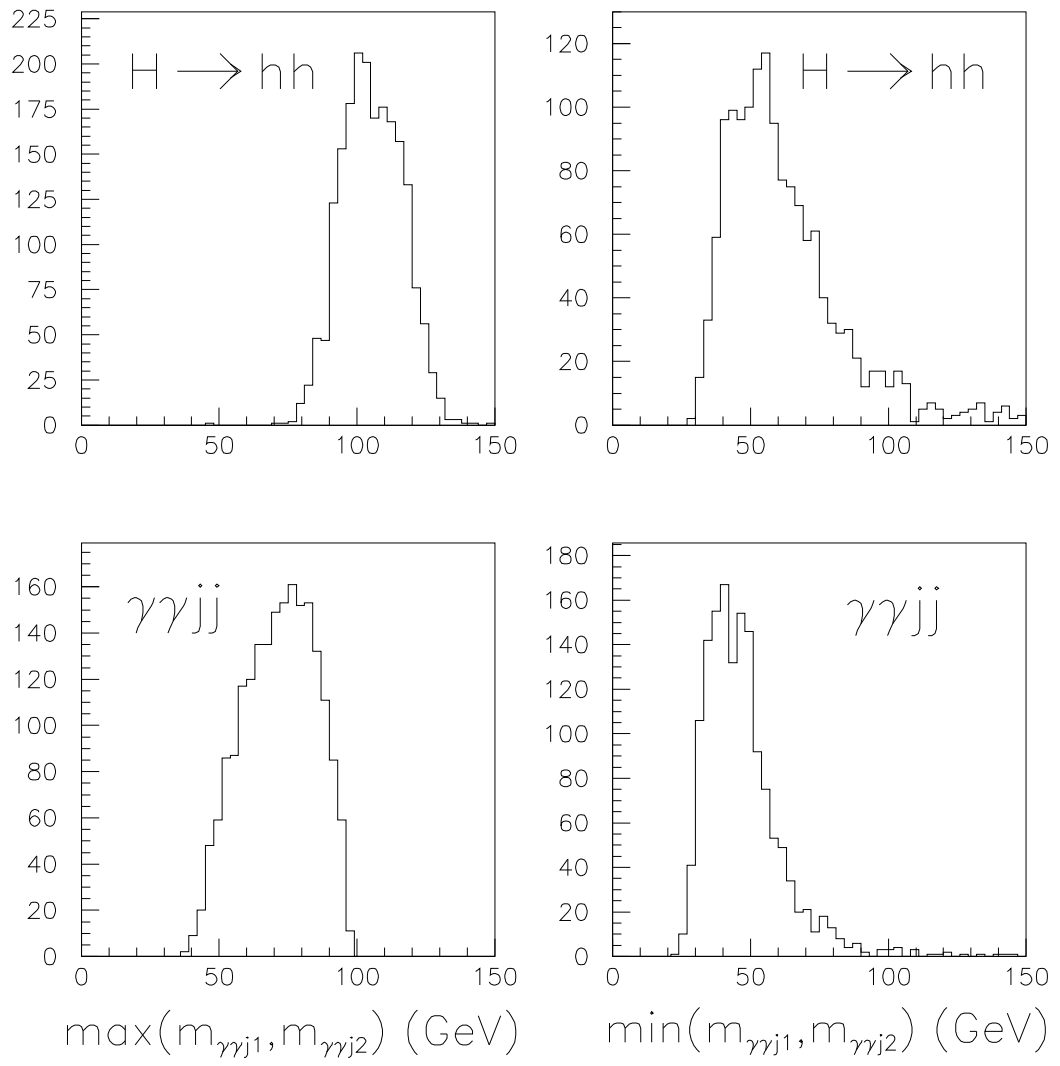

Figure 98: For $\mathrm{H} \rightarrow \mathrm{hh} \rightarrow \mathrm{b} \overline{\mathrm{b}} \gamma \gamma$ decays with $m_{\mathrm{H}}=321 \mathrm{GeV}$ and $m_{\mathrm{h}}=70 \mathrm{GeV}$ and for the residual $\gamma \gamma j j$ background, distributions of $\max \left(m_{\gamma \gamma 1}, m_{\gamma \gamma j 2}\right)$ and of $\min \left(m_{\gamma \gamma j 1}, m_{\gamma \gamma j 2}\right)$ (see text).

Table 45 shows, for $\tan \beta=1$ and for $m_{\mathrm{H}}=231 \mathrm{GeV}$ and $321 \mathrm{GeV}$, the expected signal and background acceptances and event rates after all cuts at low and high luminosity. The signal rates are small, typically from 10 to 30 events, and Poisson statistics were therefore used to estimate the expected significances (values above $\sim 8.3$ could not be reliably estimated with this method). Tables 46 and 47 summarise, for the low- and highluminosity conditions, the expected signal and total background rates, as well as the expected significances for all the values of $m_{\mathrm{H}}$ and $m_{\mathrm{h}}$ studied here. 
Table 45: For the $\mathrm{H} \rightarrow \mathrm{hh} \rightarrow \mathrm{b} \overline{\mathrm{b}} \gamma \gamma$ channel, expected signal and background acceptances and event rates at low and high luminosity, for $\tan \beta=1$ and for $m_{\mathrm{H}}=231 \mathrm{GeV}$ and $321 \mathrm{GeV}$.

\begin{tabular}{|c|c|c|c|c|}
\hline Luminosity & $3 \cdot 10^{4} \mathrm{pb}^{-1}$ & $10^{5} \mathrm{pb}^{-1}$ & $3 \cdot 10^{4} \mathrm{pb}^{-1}$ & $10^{5} \mathrm{pb}^{-1}$ \\
\hline$m_{\mathrm{H}}(\mathrm{GeV})$ & 231 & 231 & 321 & $\overline{2321}$ \\
\hline$m_{\mathrm{h}}(\mathrm{GeV})$ & 71.2 & 71.2 & 73.0 & 73.0 \\
\hline$\sigma \times B R(\mathrm{fb})$ & 6.0 & 6.0 & 4.6 & 4.6 \\
\hline Selection cuts & kinem. & kinem. & kinem & kinem. \\
\hline Acceptance & $15.3 \%$ & $6.1 \%$ & $21.3 \%$ & $13.8 \%$ \\
\hline Signal & $14.8 \pm 0.4$ & $17.6 \pm 0.7$ & $15.4 \pm 0.3$ & $29.8 \pm 0.8$ \\
\hline $\mathrm{b} \overline{\mathrm{b}} \gamma \gamma$ background & $0.04 \pm 0.04$ & $0.11 \pm 0.11$ & $0.01 \pm 0.01$ & $0.04 \pm 0.04$ \\
\hline$c \bar{c} \gamma \gamma$ background & $0.18 \pm 0.18$ & $0.12 \pm 0.12$ & $0.01 \pm 0.01$ & $0.04 \pm 0.04$ \\
\hline $\mathrm{jj} \gamma \gamma$ background & $0.95 \pm 0.06$ & $1.06 \pm 0.16$ & $0.35 \pm 0.02$ & $0.36 \pm 0.05$ \\
\hline $\mathrm{jb} \gamma \gamma$ background & $0.46 \pm 0.23$ & $0.33 \pm 0.33$ & $0.08 \pm 0.05$ & $0.11 \pm 0.11$ \\
\hline $\mathrm{jc} \gamma \gamma$ background & $0.94 \pm 0.14$ & $0.60 \pm 0.21$ & $0.23 \pm 0.04$ & $0.15 \pm 0.06$ \\
\hline Background & $2.60 \pm 0.30$ & $2.23 \pm 0.45$ & $0.69 \pm 0.07$ & $0.70 \pm 0.14$ \\
\hline Significance (Poisson) & 5.8 & 6.8 & 8.2 & $>8.3$ \\
\hline Selection cuts & $+\left(p_{T}^{h}\right)_{\max }$ & $+\left(p_{T}^{h}\right)_{\max }$ & $+\left(p_{T}^{h}\right)_{\max }$ & $+\left(p_{T}^{h}\right)_{\max }$ \\
\hline Acceptance & $14.2 \%$ & $5.8 \%$ & $16.4 \%$ & $13.6 \%$ \\
\hline Signal & $13.7 \pm 0.4$ & $16.7 \pm 0.7$ & $11.9 \pm 0.3$ & $29.44 \pm 0.8$ \\
\hline$\overline{\mathrm{b} \overline{\mathrm{b}} \gamma \gamma \text { background }}$ & $\overline{0.04 \pm 0.04}$ & $0.12 \pm 0.12$ & $0.01 \pm 0.01$ & $0.04 \pm 0.04$ \\
\hline$c \bar{c} \gamma \gamma$ background & $0.11 \pm 0.06$ & $0.12 \pm 0.12$ & $0.01 \pm 0.01$ & $0.04 \pm 0.04$ \\
\hline $\mathrm{jj} \gamma \gamma$ background & $0.44 \pm 0.04$ & $0.81 \pm 0.14$ & $0.06 \pm 0.02$ & $0.27 \pm 0.27$ \\
\hline $\mathrm{jb} \gamma \gamma$ background & $0.12 \pm 0.12$ & $0.33 \pm 0.33$ & $0.04 \pm \mathbf{0 . 0 8}$ & $0.11 \pm 0.11$ \\
\hline jc $\gamma \gamma$ background & $0.54 \pm 0.11$ & $0.38 \pm 0.17$ & $0.06 \pm 0.04$ & $0.15 \pm 0.06$ \\
\hline Background & $1.25 \pm 0.18$ & $1.75 \pm 0.43$ & $0.18 \pm 0.05$ & $0.61 \pm 0.14$ \\
\hline Significance (Poisson) & 6.4 & 7.1 & $>8.3$ & $>8.3$ \\
\hline Selection cuts & $\begin{array}{l}+\left(\mathrm{m}_{j \gamma \gamma}\right)_{\max } \\
+\left(\mathrm{m}_{j \gamma \gamma}\right)_{\text {min }}\end{array}$ & $\begin{array}{l}+\left(\mathrm{m}_{j \gamma \gamma}\right)_{\text {max }} \\
+\left(\mathrm{m}_{j \gamma \gamma}\right)_{\text {min }}\end{array}$ & $\begin{array}{l}+\left(\mathrm{m}_{j \gamma \gamma}\right)_{\max } \\
+\left(\mathrm{m}_{j \gamma \gamma}\right)_{\text {min }}\end{array}$ & $\begin{array}{l}+\left(\mathrm{m}_{j \gamma \gamma}\right)_{\max } \\
+\left(\mathrm{m}_{j \gamma \gamma}\right)_{\min }\end{array}$ \\
\hline Acceptance & $12.9 \%$ & $5.5 \%$ & $16.1 \%$ & $13.2 \%$ \\
\hline Signal & $12.5 \pm 0.3$ & $15.7 \pm 0.7$ & $11.7 \pm 0.3$ & $28.4 \pm 0.8$ \\
\hline $\mathrm{bb} \gamma \gamma$ background & $0.04 \pm 0.04$ & $0.12 \pm 0.12$ & $0.01 \pm 0.01$ & $0.04 \pm 0.04$ \\
\hline$c \bar{c} \gamma \gamma$ background & $0.11 \pm 0.06$ & $0.12 \pm 0.12$ & $0.01 \pm 0.01$ & $0.04 \pm 0.04$ \\
\hline $\mathrm{jj} \gamma \gamma$ background & $0.33 \pm 0.03$ & $0.58 \pm 0.12$ & $0.04 \pm 0.01$ & $0.13 \pm 0.03$ \\
\hline $\mathrm{jb} \gamma \gamma$ background & $0.12 \pm 0.12$ & $0.33 \pm 0.33$ & $0.04 \pm 0.04$ & $0.11 \pm 0.11$ \\
\hline jc $\gamma \gamma$ background & $0.44 \pm 0.10$ & $0.23 \pm 0.13$ & $0.05 \pm 0.02$ & $0.12 \pm 0.06$ \\
\hline Background & $1.00 \pm 0.17$ & $1.37 \pm 0.41$ & $0.15 \pm 0.05$ & $0.44 \pm \mathbf{0 . 1 4}$ \\
\hline Significance (Poisson) & 6.4 & 7.3 & $>8.3$ & $>8.3$ \\
\hline
\end{tabular}


Table 46: Observability of the $\mathrm{H} \rightarrow \mathrm{hh} \rightarrow \mathrm{b} \overline{\mathrm{b}} \gamma \gamma$ channel for an integrated luminosity of $3 \cdot 10^{4} \mathrm{pb}^{-1}$ and for several values of $m_{\mathrm{H}}$ and $m_{\mathrm{h}}$. The $\sigma \times B R$ values and the expected numbers of signal and background events are given for $m_{\mathrm{h}} \sim 72 \mathrm{GeV}(\tan \beta=1)$ and for $m_{\mathrm{h}} \sim 97 \mathrm{GeV}(\tan \beta=3)$.

\begin{tabular}{|c||c|c|c|c|}
\hline$m_{\mathrm{H}}(\mathrm{GeV})$ & 231 & 275 & 321 & 368 \\
\hline$m_{\mathrm{h}}(\mathrm{GeV})$ & 71.2 & 72.4 & 73.0 & 73.4 \\
\hline$\sigma \times B R(\mathrm{fb})$ & $\mathbf{6 . 0}$ & $5 . \mathbf{0}$ & 4.6 & $\mathbf{0 . 9}$ \\
\hline \hline Signal & $13.7 \pm \mathbf{0 . 4}$ & $11.9 \pm \mathbf{0 . 3}$ & $11.9 \pm \mathbf{0 . 3}$ & $3.4 \pm \mathbf{0 . 1}$ \\
\hline Background & $1.25 \pm \mathbf{0 . 1 8}$ & $\mathbf{0 . 2 2 \pm \mathbf { 0 . 0 5 }}$ & $\mathbf{0 . 1 8} \pm \mathbf{0 . 0 5}$ & $\mathbf{0 . 1 2} \pm \mathbf{0 . 0 5}$ \\
\hline Significance (Poisson) & $\mathbf{6 . 4}$ & $>8.3$ & $>8.3$ & 3.6 \\
\hline \hline$m_{\mathrm{H}}(\mathrm{GeV})$ & 210 & 258 & 306 & 355 \\
\hline$m_{\mathrm{h}}(\mathrm{GeV})$ & $\mathbf{9 5 . 5}$ & $\mathbf{9 7 . 4}$ & $\mathbf{9 8 . 3}$ & $\mathbf{9 8 . 8}$ \\
\hline $\boldsymbol{\sigma} \times$ BR $(\mathrm{fb})$ & 2.0 & 1.6 & 1.2 & 1.0 \\
\hline Signal & $4.9 \pm \mathbf{0 . 1 2}$ & $3.7 \pm \mathbf{0 . 1}$ & $3.5 \pm \mathbf{0 . 1}$ & $3.3 \pm \mathbf{0 . 1}$ \\
\hline Background & $3.2 \pm \mathbf{0 . 4 0}$ & $1.1 \pm \mathbf{0 . 1}$ & $\mathbf{0 . 6} \pm \mathbf{0 . 1}$ & $\mathbf{0 . 4} \pm \mathbf{0 . 1}$ \\
\hline Significance (Poisson) & 2.1 & 2.0 & 2.7 & 2.4 \\
\hline
\end{tabular}

Table 47: Observability of the $\mathrm{H} \rightarrow \mathrm{hh} \rightarrow \mathrm{b} \overline{\mathrm{b}} \gamma \gamma$ channel for an integrated luminosity of $10^{5} \mathrm{pb}^{-1}$ and for several values of $m_{\mathrm{H}}$ and $m_{\mathrm{h}}$. The $\sigma \times B R$ values and the expected numbers of signal and background events are given for $m_{\mathrm{h}} \sim 72 \mathrm{GeV}(\tan \beta=1)$ and for $m_{\mathrm{h}} \sim 97 \mathrm{GeV}(\tan \beta=3)$.

\begin{tabular}{|c||c|c|c|c|}
\hline$m_{\mathrm{H}}(\mathrm{GeV})$ & 231 & 275 & 321 & 368 \\
\hline$m_{\mathrm{h}}(\mathrm{GeV})$ & 71.2 & 72.4 & 73.0 & 73.4 \\
\hline$\sigma \times B R(\mathrm{fb})$ & $\mathbf{6 . 0}$ & $5 . \mathbf{0}$ & 4.6 & $\mathbf{0 . 9}$ \\
\hline Signal & $15.7 \pm \mathbf{0 . 7}$ & $21.1 \pm \mathbf{0 . 7}$ & $28.4 \pm \mathbf{0 . 8}$ & $7.6 \pm \mathbf{0 . 2}$ \\
\hline Background & $1.4 \pm \mathbf{0 . 4}$ & $\mathbf{0 . 5} \pm \mathbf{0 . 1 4}$ & $\mathbf{0 . 4} \pm \mathbf{0 . 2}$ & $\mathbf{0 . 3} \pm \mathbf{0 . 1}$ \\
\hline Significance (Poisson) & 7.3 & $>8.3$ & $>8.3$ & 5.4 \\
\hline \hline$m_{\mathrm{H}}(\mathrm{GeV})$ & 210 & 258 & 306 & 355 \\
\hline$m_{\mathrm{h}}(\mathrm{GeV})$ & $\mathbf{9 5 . 5}$ & $\mathbf{9 7 . 4}$ & $\mathbf{9 8 . 3}$ & $\mathbf{9 8 . 8}$ \\
\hline $\boldsymbol{\sigma} \times B R(\mathrm{fb})$ & $\mathbf{2 . 0}$ & 1.6 & 1.2 & 1.0 \\
\hline Signal & $4.6 \pm \mathbf{0 . 2}$ & $4.3 \pm \mathbf{0 . 2}$ & $5.8 \pm \mathbf{0 . 2}$ & $\mathbf{6 . 8} \pm \mathbf{0 . 2}$ \\
\hline Background & $2.1 \pm \mathbf{0 . 5}$ & $\mathbf{0 . 8} \pm \mathbf{0 . 2}$ & $1 . \mathbf{0} \pm \mathbf{0 . 2}$ & $\mathbf{0 . 7} \pm \mathbf{0 . 2}$ \\
\hline Significance (Poisson) & 2.1 & 3.0 & 3.2 & 4.2 \\
\hline
\end{tabular}




\section{The $\mathrm{H} / \mathrm{A} \rightarrow \mathrm{t} \overline{\mathrm{t}}$ channel}

As discussed in Section 5.7, the $\mathrm{H} \rightarrow \mathrm{t} \overline{\mathrm{t}}$ and $\mathrm{A} \rightarrow \mathrm{t} \overline{\mathrm{t}}$ branching ratios are close to $100 \%$ for $m_{\mathrm{H}}, m_{\mathrm{A}}>2 m_{\mathrm{t}}$ and for $\tan \beta \sim 1$. The $\mathrm{H} \rightarrow \mathrm{t} \overline{\mathrm{t}}$ and $\mathrm{A} \rightarrow \mathrm{t} \overline{\mathrm{t}}$ decays cannot be distinguished experimentally from each other, since the $\mathrm{H}$ - and A-bosons are almost degenerate in mass in the relevant region of parameter space.

The signal was extracted by searching for $W W b \bar{b}$ final states, with one $\mathrm{W} \rightarrow \ell \nu$ decay, which provides the trigger, and one hadronic $\mathrm{W} \rightarrow \mathrm{jj}$ decay. The lepton was required to have $|\eta|<2.5$ and $\mathrm{p}_{\mathrm{T}}>20 \mathrm{GeV}$. The jets from $\mathrm{W} \rightarrow \mathrm{jj}$ decay were required to have $|\eta|<3.0$ and $\mathrm{p}_{\mathrm{T}}>40 \mathrm{GeV}$. The two b-jets were required to have $|\eta|<3.0$ and $\mathrm{p}_{\mathrm{T}}>40 \mathrm{GeV}$ and to both be tagged, with an assumed efficiency $\epsilon_{b}=60 \%$ (resp. 50\%) at low (resp. high) luminosity.

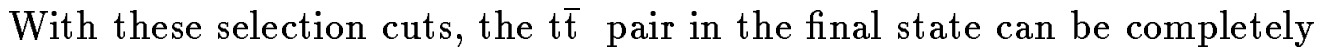
reconstructed with good efficiency, both at low and high luminosity, and it is therefore reasonable to assume that the backgrounds to the signal from non-

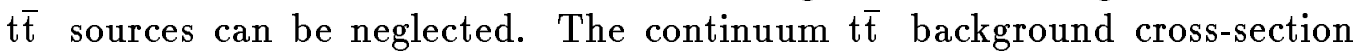
depends on $m_{\mathrm{t}}$ and decreases from $490 \mathrm{pb}$ for $m_{\mathrm{t}}=150 \mathrm{GeV}$, to $252 \mathrm{pb}$ for $m_{\mathrm{t}}=175 \mathrm{GeV}$, and to $140 \mathrm{pb}$ for $m_{\mathrm{t}}=200 \mathrm{GeV}$, where the values quoted include the $\mathrm{W} \rightarrow \ell \nu$ decay branching ratio.

The selection cuts described above have an acceptance of $\sim 10 \%$ for both the signal and the background. For events which pass these cuts, the masses of both top-quarks are reconstructed by using the energy of the lepton and of the jets in the final state, combined with the $E_{T}^{\text {miss }}$ measurement obtained from the whole calorimeter. The $\mathrm{W} \rightarrow \ell \nu$ decay is fully reconstructed, by using, in addition to the trigger lepton and the measured $E_{T}^{\text {miss }}$, a constraint on $m_{\mathrm{W}}$ to determine the longitudinal momentum of the escaping neutrino.

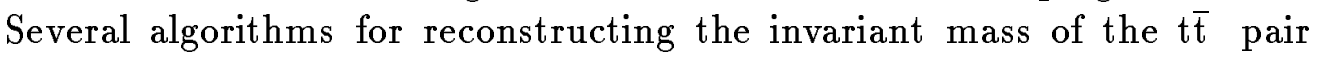
have been studied, in order to understand how to minimise the combinatorial background. The best algorithm to-date turned out to be the one where the $\ell \nu b$ and $j j b$ combinations were chosen, which simultaneously optimised the reconstruction of both top-quark masses, i.e. which minimised the quantity

$\chi_{\mathrm{t} \overline{\mathrm{t}}}^{2}=\left(m_{\mathrm{t}}-m_{b \ell \nu}\right)^{2} / \sigma_{m_{b \ell \nu}}^{2}+\left(m_{\mathrm{t}}-m_{b j j}\right)^{2} / \sigma_{m_{b j j}}^{2}$.

This method does not change the $t \bar{t}$ mass spectrum for the background and provides the best mass resolution for the Higgs-boson signal. Fig. 99 shows the distributions for these reconstructed masses, as obtained for $\mathrm{H} \rightarrow \mathrm{t} \overline{\mathrm{t}}$ decays with $m_{\mathrm{H}}=400 \mathrm{GeV}$ and for the $\mathrm{t} \overline{\mathrm{t}}$ continuum. As expected, the mass resolution is worse for the $t \rightarrow b \ell \nu$ decays, because of the poor accuracy on the extraction of the longitudinal momentum of the neutrino. The combinatorial background under the $t \rightarrow b j j$ decays is larger for the $\mathrm{H} \rightarrow \mathrm{t} \overline{\mathrm{t}}$ signal than for the $\mathrm{t} \overline{\mathrm{t}}$ continuum. 

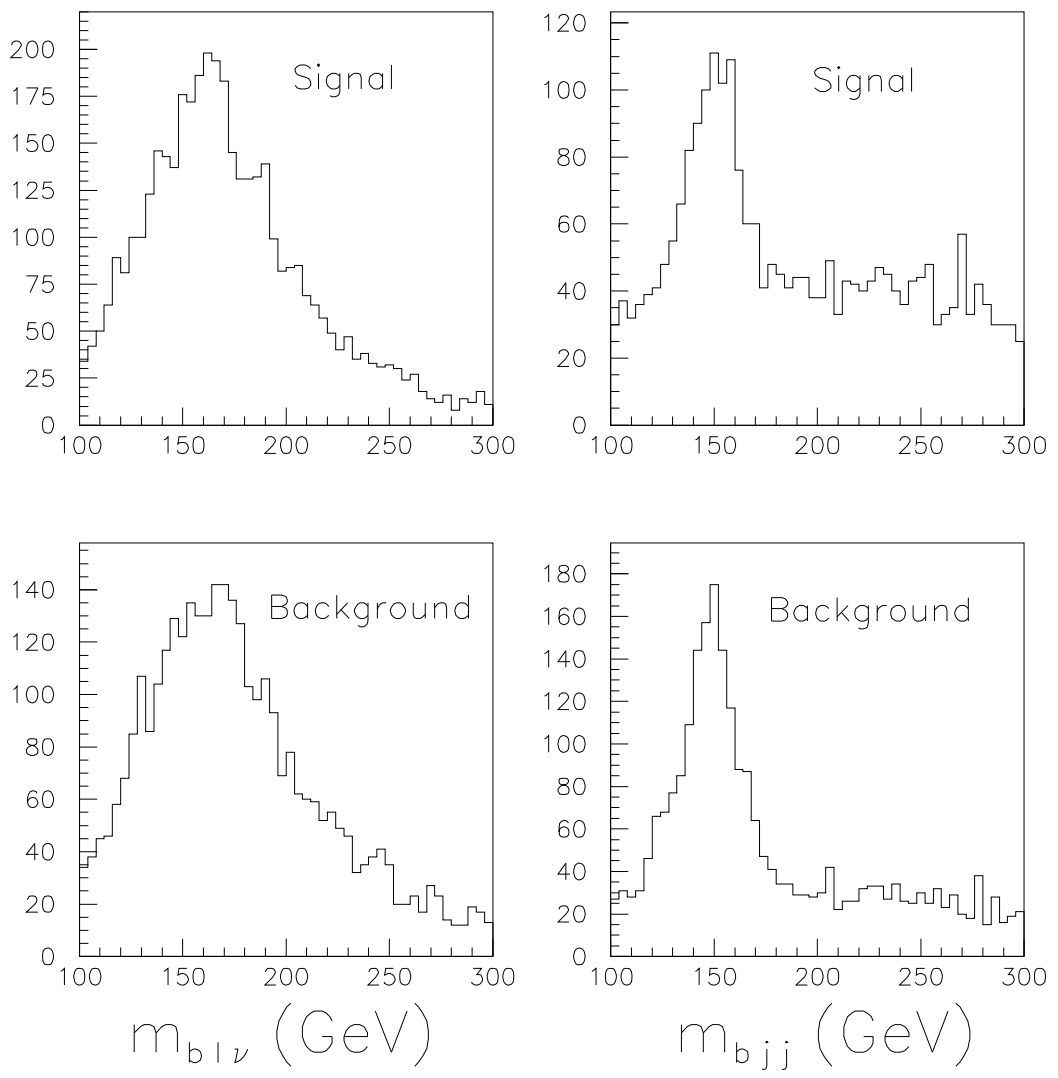

Figure 99: For $\mathrm{H} \rightarrow \mathrm{t} \overline{\mathrm{t}}$ decays with $m_{\mathrm{H}}=400 \mathrm{GeV}$ (top) and for the $\mathrm{t} \overline{\mathrm{t}}$ continuum background (bottom), reconstructed invariant mass distributions, $m_{b}$ (left), for $t \rightarrow b \ell \nu$ decays, and $m_{b j j}$ (right), for $t \rightarrow b j j$ decays.

Once both top-quarks are reconstructed, their 4-vectors can be rescaled by applying a constraint on $m_{\mathrm{t}}$ before reconstructing $m_{\mathrm{H}}$. Fig. 100 shows the distributions for the reconstructed $t \bar{t}$ mass before and after applying the constraint on $m_{\mathrm{t}}$, as obtained for $\mathrm{H} \rightarrow \mathrm{t} \overline{\mathrm{t}}$ decays with $m_{\mathrm{H}}=400 \mathrm{GeV}$ and for the $t \bar{t}$ continuum. The mass resolution improves significantly for the signal after the constraint on $m_{\mathrm{t}}$, but the background distribution also tends to peak towards lower masses. Even after applying the constraint on $m_{\mathrm{t}}$, the signal mass resolution is quite large and varies from $\sim 35$ to $\sim 80 \mathrm{GeV}$ if $m_{\mathrm{H}}$ increases from 330 to $500 \mathrm{GeV}$. 

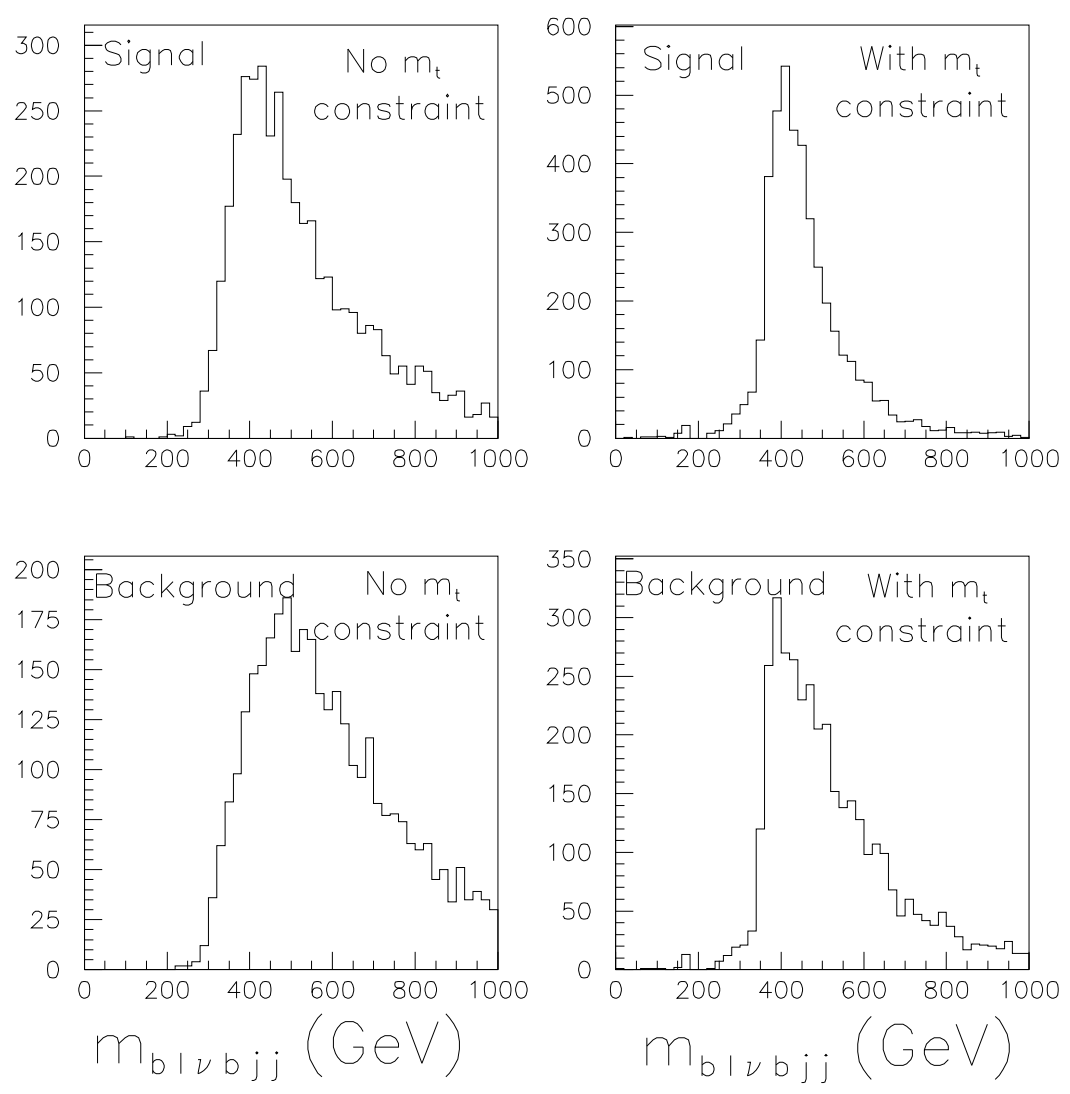

Figure 100: For $\mathrm{H} \rightarrow \mathrm{t} \overline{\mathrm{t}}$ decays with $m_{\mathrm{H}}=400 \mathrm{GeV}$ (top) and for the t $\mathrm{t} \overline{\mathrm{t}}$ continuum background (bottom), reconstructed invariant mass distributions of the $\mathrm{t} \overline{\mathrm{t}}$ pair, $m_{b}{ }_{b j j}$, before (left) and after (right) applying a constraint on $m_{\mathrm{t}}$.

Table 48 shows the overall acceptances for the signal and background as a function of $m_{\mathrm{H}}, m_{\mathrm{A}}$ and for three different values of $m_{\mathrm{t}}$. These acceptances include a lepton reconstruction efficiency of $90 \%$, the b-tagging efficiency quoted above, the acceptance of the selection cuts and of the chosen mass bin as presented in the Table. The use of the constraint on $m_{t}$, as implemented crudely for this study, results in non-negligible systematic shifts of the reconstructed $t \bar{t}$ mass peak with respect to the Higgs boson mass. 
Table 48: Acceptance of all selection cuts for the $H / \mathrm{A} \rightarrow \mathrm{t} \overline{\mathrm{t}}$ signal and for the $\mathrm{t} \overline{\mathrm{t}}$ background as a function of $m_{\mathrm{H}}, m_{\mathrm{A}}$ for three different values of $m_{\mathrm{t}}$. The numbers include the lepton reconstruction and b-tagging efficiencies.

\begin{tabular}{|c||c|c|c|}
\hline \multicolumn{1}{|c|}{$m_{\mathrm{A}}, m_{\mathrm{H}}(\mathrm{GeV})$} & Mass bin $(\mathrm{GeV})$ & Signal acceptance & Background acceptance \\
\hline \hline & \multicolumn{3}{|c|}{$m_{\mathrm{t}}=150 \mathrm{GeV}$} \\
\hline \hline 330 & $360 \pm 70$ & $2.7 \%$ & $2.0 \%$ \\
\hline 370 & $390 \pm 70$ & $3.4 \%$ & $2.0 \%$ \\
\hline 400 & $410 \pm 80$ & $4.1 \%$ & $2.2 \%$ \\
\hline 450 & $435 \pm 120$ & $5.7 \%$ & $2.8 \%$ \\
\hline 500 & $460 \pm 160$ & $7.5 \%$ & $3.4 \%$ \\
\hline \hline & & $m_{\mathrm{t}}=175 \mathrm{GeV}$ \\
\hline \hline 370 & $390 \pm 70$ & $3.8 \%$ & $2.8 \%$ \\
\hline 400 & $410 \pm 80$ & $4.9 \%$ & $3.5 \%$ \\
\hline 450 & $435 \pm 120$ & $7.0 \%$ & $5.4 \%$ \\
\hline 500 & $460 \pm 160$ & $8.7 \%$ & $4.7 \%$ \\
\hline \hline & & $m_{\mathrm{t}}=200 \mathrm{GeV}$ & $5.9 \%$ \\
\hline \hline 450 & $435 \pm 120$ & $7.3 \%$ & $9.7 \%$ \\
\hline 500 & $460 \pm 160$ & \multicolumn{3}{|c|}{} \\
\hline
\end{tabular}

Table 49 shows the expected signal and background rates for integrated luminosities of $3 \cdot 10^{4} \mathrm{pb}^{-1}$ and $10^{5} \mathrm{pb}^{-1}$, as a function of $m_{\mathrm{H}}, m_{\mathrm{A}}$ and for

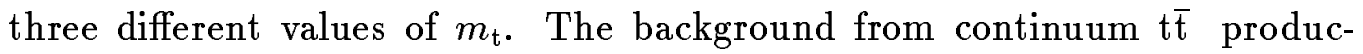
tion is unfortunately much larger than the signal; the signal-to-background ratio varies between $1.5 \%$ and $7 \%$ over the range of Higgs-boson and topquark masses considered. Given the large t $\overline{\mathrm{t}}$ mass bins needed to collect the signal (see Table 48), it is clear that the signal can only be observed above the continuum background as an excess of events. This excess would be very significant statistically, as shown in Table 49 , but the quoted significances would only be meaningful if the theoretical uncertainties on the continuum background shape were lower than a percent or so. Fig. 101 illustrates this problem by displaying the expected summed signal+background reconstructed t $\overline{\mathrm{t}}$ mass distribution, for $m_{\mathrm{H}}, m_{\mathrm{A}}=400 \mathrm{GeV}$ and for an integrated luminosity of $3 \cdot 10^{4} \mathrm{pb}^{-1}$ (see Section 5.7 for a further discussion of this point). 
Table 49: Observability of the $H / \mathrm{A} \rightarrow \mathrm{t} \overline{\mathrm{t}}$ channel at low and high luminosities. The $\sigma \times B R$ values and the expected numbers of signal and background events are given for combined $H / \mathrm{A} \rightarrow \mathrm{t} \overline{\mathrm{t}}$ decays and for $\tan \beta=1.5$.

\begin{tabular}{|c|c|c|c|c|c|c|c|}
\hline & \multirow{2}{*}{\multicolumn{3}{|c|}{$\frac{\text { Low luminosity }}{3 \cdot 10^{4} \mathrm{pb}^{-1}}$}} & \multirow{2}{*}{\multicolumn{3}{|c|}{$\frac{\text { High luminosity }}{10^{5} \mathrm{pb}^{-1}}$}} \\
\hline & & & & & & & \\
\hline $\begin{array}{c}m_{\mathrm{H}}, m_{\mathrm{A}} \\
(\mathrm{GeV}) \\
\end{array}$ & $\begin{array}{c}\sigma \times B R \\
(\mathrm{pb})\end{array}$ & Signal & Background & Signif. & Signal & Background & Signif. \\
\hline & & \multicolumn{6}{|c|}{$m_{\mathrm{t}}=150 \mathrm{GeV}$} \\
\hline 330.0 & 10.5 & 2750 & 95600 & 8.9 & 6370 & 220500 & 13.6 \\
\hline 370.0 & 8.0 & 2650 & 95300 & 8.6 & 6130 & 220500 & 12.9 \\
\hline 400.0 & 6.2 & 2460 & 104800 & 7.6 & 5700 & 242500 & 11.4 \\
\hline 450.0 & 4.0 & 2220 & 133400 & 6.0 & 5130 & 308700 & 9.20 \\
\hline \multirow[t]{2}{*}{500.0} & 2.9 & 2120 & 161900 & 5.3 & 4900 & 374800 & 7.90 \\
\hline & & \multicolumn{6}{|c|}{$m_{\mathrm{t}}=175 \mathrm{GeV}$} \\
\hline 370.0 & 11.8 & 4360 & 68600 & 16.7 & 10100 & 158700 & 25.3 \\
\hline 400.0 & 8.40 & 4000 & 85700 & 13.7 & 9270 & 198500 & 20.9 \\
\hline 450.0 & 4.80 & 3270 & 107800 & 10.0 & 7570 & 249500 & 15.2 \\
\hline \multirow[t]{2}{*}{500.0} & 2.90 & 2450 & 127400 & 6.9 & 5670 & 294800 & 10.4 \\
\hline & & \multicolumn{6}{|c|}{$m_{\mathrm{t}}=200 \mathrm{GeV}$} \\
\hline 450.0 & 6.0 & 4260 & 63400 & 16.9 & 9870 & 146700 & 25.6 \\
\hline 500.0 & 3.8 & 3590 & 79700 & 12.7 & 8300 & 184500 & 19.3 \\
\hline
\end{tabular}

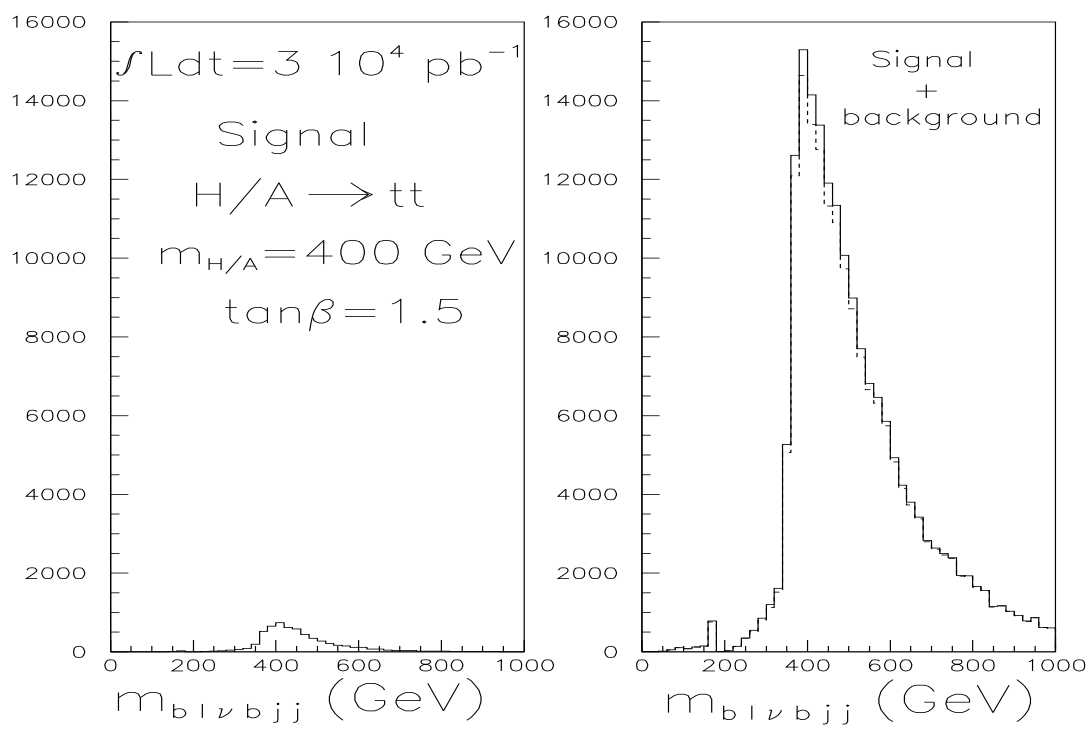

Figure 101: For an integratedluminosity of $3 \cdot 10^{4} \mathrm{pb}^{-1}$ and for $\tan \beta=1.5$, reconstructed $\mathrm{t} \overline{\mathrm{t}}$ mass distributions, $m_{b}$ bjj, for the combined $H / \mathrm{A} \rightarrow \mathrm{t} \overline{\mathrm{t}}$ signal with $m_{\mathrm{H}}=400 \mathrm{GeV}$ (left) and for the continuum $\mathrm{t} \overline{\mathrm{t}}$ background (dashed right histogram) and summed signal+background (solid right histogram). 


\section{$\mathrm{E} \quad$ The $\mathrm{A} \rightarrow \mathrm{Zh} \rightarrow \ell \ell \mathrm{b} \overline{\mathrm{b}}$ channel}

The observation of this channel would be particularly interesting, since it would correspond to the simultaneous discovery of two Higgs bosons. It is the dominant A-boson decay channel for low values of $\tan \beta$ and for $m_{\mathrm{Z}}+m_{\mathrm{h}}<m_{\mathrm{A}}<2 m_{\mathrm{t}}$. The $\mathrm{A} \rightarrow \mathrm{Zh} \rightarrow \ell \ell \mathrm{b} \overline{\mathrm{b}}$ channel was the only one studied here, because it can be easily triggered upon and it offers the largest rates apart from the dominant $\mathrm{A} \rightarrow \mathrm{Zh} \rightarrow \mathrm{b} \overline{\mathrm{b}} \mathrm{b} \overline{\mathrm{b}}$ channel (see Section 5.8).

Several background sources were considered: irreducible $\mathrm{Zb} \overline{\mathrm{b}} \rightarrow \ell \ell \mathrm{b} \overline{\mathrm{b}}$ and $\mathrm{ZZ} \rightarrow \ell \ell \mathrm{b} \overline{\mathrm{b}}$ on the one hand, and reducible $\mathrm{ZW} \rightarrow \ell \ell \mathrm{jj}, \mathrm{Zjj} \rightarrow \ell \ell \mathrm{jj}$ and $\mathrm{t} \overline{\mathrm{t}} \rightarrow \mathrm{WWb} \overline{\mathrm{b}}$, with both $\mathrm{W} \rightarrow \ell \nu$, on the other hand. Table 50 shows the expected cross-sections, including branching ratios, for all these backgrounds after the cuts used at event generation. The two dominant backgrounds are the irreducible $\mathrm{Zb} \overline{\mathrm{b}}$, which was generated using the EKS matrix-element

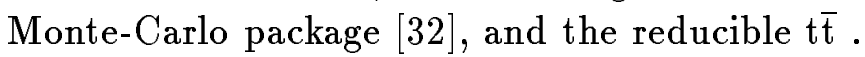

Table 50: Expected cross-sections, including branching ratios, after cuts used at event generation, for the $\mathrm{Zb} \overline{\mathrm{b}}, \mathrm{ZZ}, \mathrm{ZW}, \mathrm{Zjj}$ and $\mathrm{t} \overline{\mathrm{t}}$ backgrounds to the $\mathrm{A} \rightarrow \mathrm{Zh} \rightarrow \ell \mathrm{lb} \overline{\mathrm{b}}$ signal.

\begin{tabular}{|c||c|c|}
\hline Process & Cuts at generation & $\sigma \times B R(\mathrm{pb})$ \\
\hline \hline $\mathrm{Zb} \overline{\mathrm{b}}$ & & 36 \\
\hline $\mathrm{ZZ} \mathrm{with} \mathrm{Z} \rightarrow \mathrm{b} \overline{\mathrm{b}}$ & $\sqrt{\hat{s}}>150 \mathrm{GeV}$ & 0.22 \\
\hline $\mathrm{ZW}$ with $\mathrm{W} \rightarrow \mathrm{jj}$ & $\sqrt{\hat{s}}>150 \mathrm{GeV}$ & 1.16 \\
\hline $\mathrm{Zjj}$ & $\sqrt{\hat{s}}>80 \mathrm{GeV}$ & 1880 \\
& $\mathrm{p}_{\mathrm{T}}^{\text {hard }}>10 \mathrm{GeV}$ & \\
\hline $\mathrm{t} \overline{\mathrm{t}}$ & $m_{\mathrm{t}}=150 \mathrm{GeV}$ & 54.4 \\
$\mathrm{t} \overline{\mathrm{t}}$ & $m_{\mathrm{t}}=175 \mathrm{GeV}$ & 28.0 \\
$\mathrm{t} \overline{\mathrm{t}}$ & $m_{\mathrm{t}}=200 \mathrm{GeV}$ & 15.6 \\
\hline
\end{tabular}

The signal was extracted by requiring two isolated leptons, with $|\eta|<2.5$ and $\mathrm{p}_{\mathrm{T}}>20 \mathrm{GeV}$, and two additional jets with $|\eta|<2.5$ and $\mathrm{p}_{\mathrm{T}}>15 \mathrm{GeV}$ (resp. $\mathrm{p}_{\mathrm{T}}>30 \mathrm{GeV}$ ) at low (resp. high) luminosity. Both jets were required to be tagged as b-jets with an assumed efficiency $\epsilon_{b}=60 \%$ (resp. $50 \%$ ) at low (resp. high) luminosity. Events were accepted if the dilepton mass was within $\pm 6 \mathrm{GeV}$ of $m_{\mathrm{Z}}$, and if the dijet mass was within $\pm 20 \mathrm{GeV}$ of $m_{\mathrm{h}}-$ $20 \mathrm{GeV}$ (no correction to the reconstructed dijet mass was applied in this study). Finally, after rescaling the lepton and jet 4-momenta appropriately by applying constraints on $m_{\mathrm{Z}}$ and $m_{\mathrm{h}}$, the $\ell \ell \mathrm{jj}$ invariant mass was required to be within $\pm 6 \mathrm{GeV}$ of $m_{\mathrm{A}}$. Figure 102 shows the reconstructed $m_{\ell \ell}$ and $m_{\mathrm{b} \overline{\mathrm{b}}}$ mass distributions for the signal with $m_{\mathrm{A}}=300 \mathrm{GeV}$ and $m_{\mathrm{h}}=70 \mathrm{GeV}$ and for the dominant $\mathrm{Zb} \overline{\mathrm{b}}$ and $\mathrm{t} \overline{\mathrm{t}}$ backgrounds. The low-energy tail discussed in Appendix A.3 is clearly visible in the $m_{\mathrm{b} \overline{\mathrm{b}}}$ distribution. 
Figure 102 also shows the reconstructed $m_{\ell \ell b \bar{b}}$ distribution after rescaling the lepton and b-jet 4-momenta appropriately by applying constraints on $m_{\mathrm{Z}}$ and $m_{\mathrm{h}}$, which improve the overall mass resolution to $\sim 6 \mathrm{GeV}$ and reduce significantly the fraction of non-Gaussian tails. The overall acceptance for the signal with $m_{\mathrm{A}}=300 \mathrm{GeV}$ is $\sim 7 \%$ (resp. $\sim 3.4 \%$ ) at low (resp. high) luminosity, including a lepton reconstruction efficiency of $90 \%$, the b-tagging efficiency quoted above, the acceptance of the selection cuts and of the chosen mass bins.
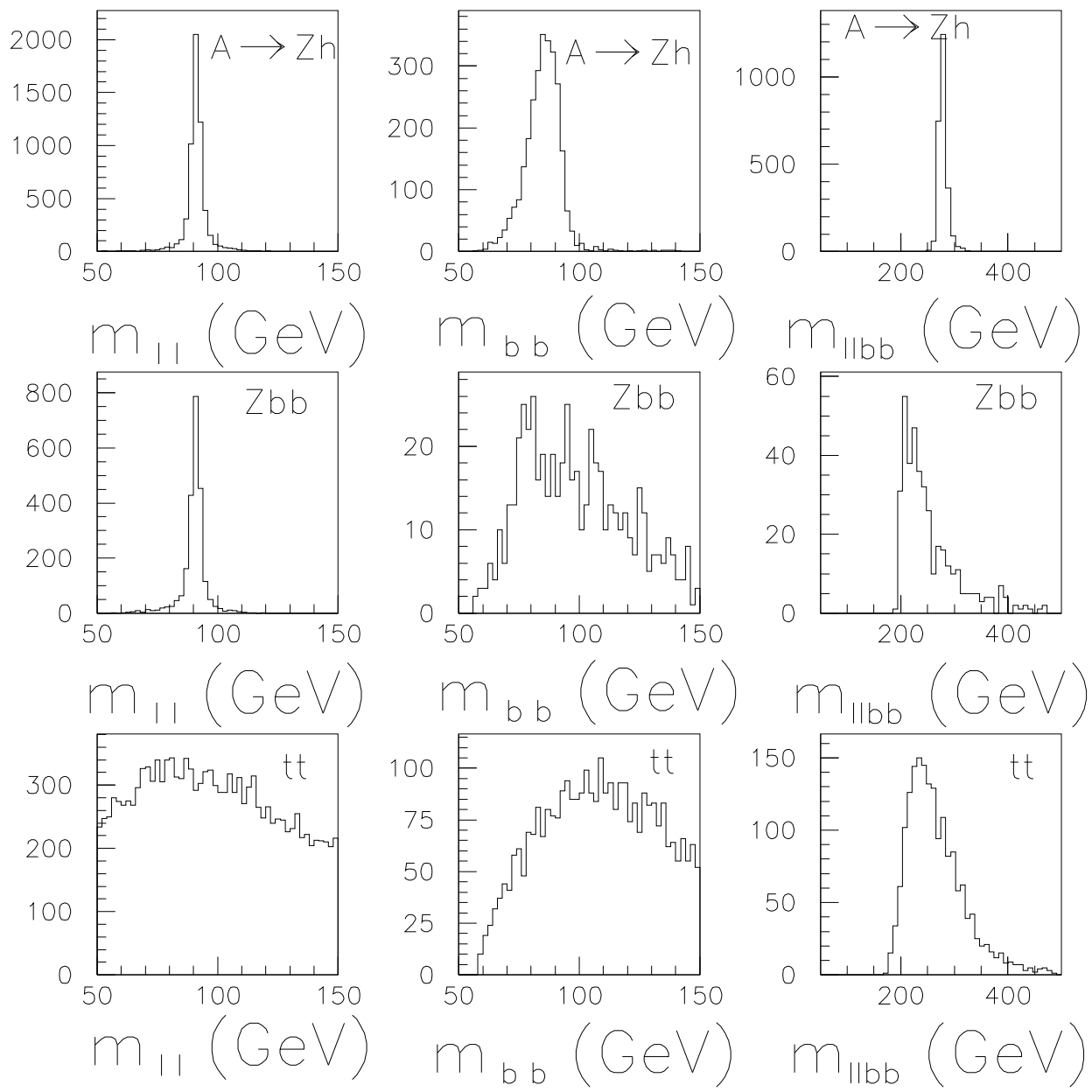

Figure 102: For $\mathrm{A} \rightarrow \mathrm{Zh} \rightarrow \ell \mathrm{hb} \overline{\mathrm{b}}$ decays with $m_{\mathrm{A}}=300 \mathrm{GeV}$ and $m_{\mathrm{h}}=70 \mathrm{GeV}$ and for the dominant $\mathrm{Zb} \overline{\mathrm{b}}$ and $\mathrm{t} \overline{\mathrm{t}}$ backgrounds, reconstructed mass distributions after all selection cuts for $m_{\ell \ell}, m_{\mathrm{b} \overline{\mathrm{b}}}$ and $m_{\ell \ell \mathrm{b} \overline{\mathrm{b}}}$ (after using the $m_{\mathrm{Z}}$ and $m_{\mathrm{h}}$ mass constraints). 
Tables 51 and 52 give the expected numbers of signal and background events, as well as the expected significances, for low and high luminosity respectively. The signal rates are given for $\tan \beta=1$ and for $m_{\mathrm{A}}=200,250$ and $300 \mathrm{GeV}$, corresponding to $m_{\mathrm{h}}=71,72$ and $73 \mathrm{GeV}$, respectively. The background rates are given separately for each process and, in the case of $t \bar{t}$, for three values of $m_{\mathrm{t}}$. The rejection of non-b-jets was assumed to be $\sim 100$ (resp. $\sim 50$ ) at low (resp. high) luminosity. The ZW background, with $\mathrm{Z} \rightarrow \ell \ell$ and $\mathrm{W} \rightarrow \mathrm{jj}$, is at the level of only a few percent of the Zjj background, with $\mathrm{Z} \rightarrow \ell \ell$, and can therefore be neglected.
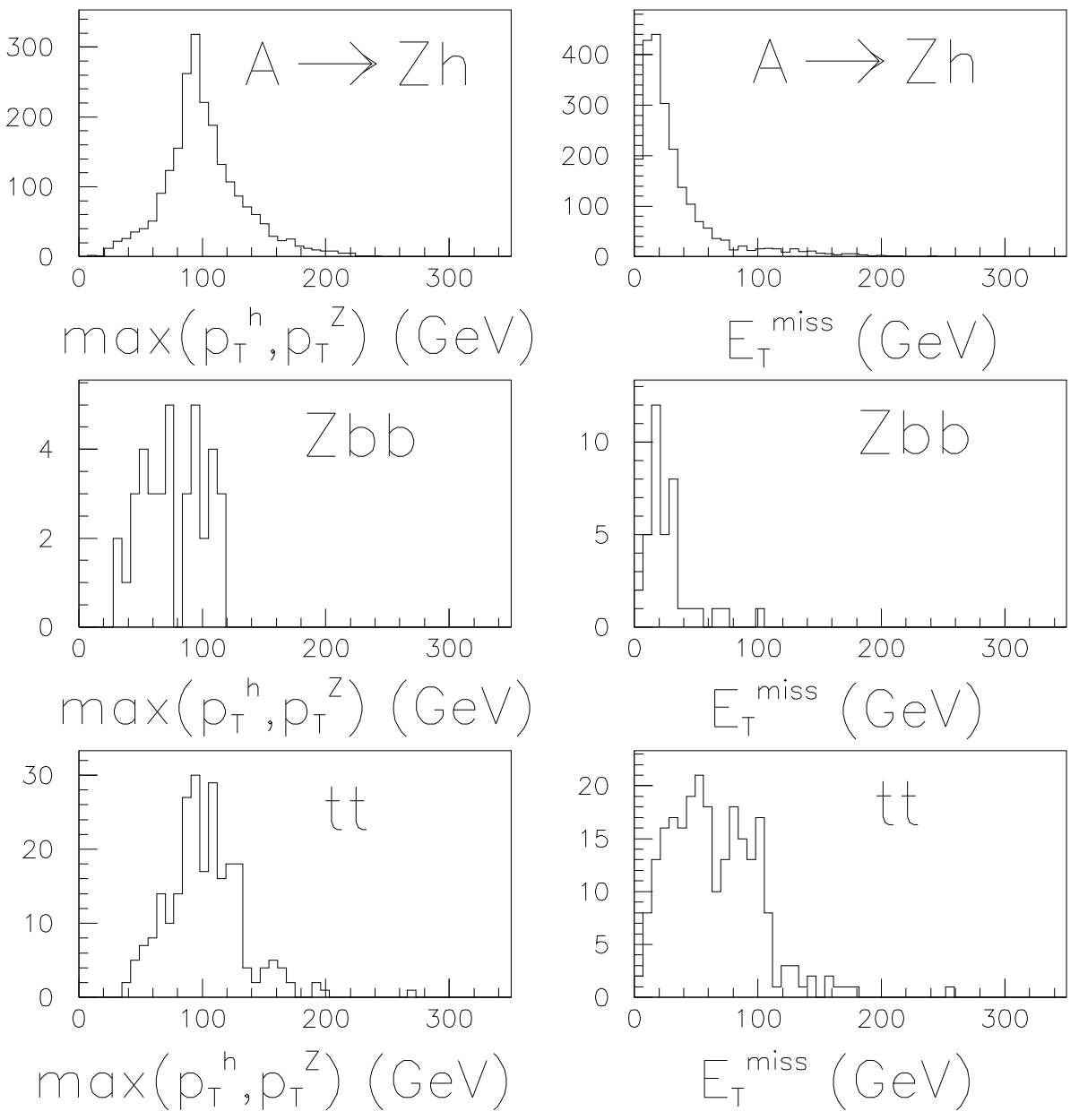

Figure 103: For $\mathrm{A} \rightarrow \mathrm{Zh} \rightarrow \ell \mathrm{hb} \overline{\mathrm{b}}$ decays with $m_{\mathrm{A}}=300 \mathrm{GeV}$ and $m_{\mathrm{h}}=70 \mathrm{GeV}$ and for the dominant $\mathrm{Zb} \overline{\mathrm{b}}$ and $\mathrm{t} \overline{\mathrm{t}}$ backgrounds, distributions of $\max \left(p_{\mathrm{T}}{ }^{h}, p_{\mathrm{T}}{ }^{Z}\right)$ and $E_{T}^{\mathrm{miss}}$ after all selection cuts, including the mass cuts. 
Table 51 shows that the dominant sources of background arise from $\mathrm{Zb} \overline{\mathrm{b}}$ and $t \bar{t}$ production. The signal-to-background ratio increases from $\sim 1 / 2$ (resp. $\sim 3 / 1$ ) to $\sim 1 / 1$ (resp. $\sim 4 / 1$ ) for $m_{\mathrm{A}}=200 \mathrm{GeV}$ (resp. $300 \mathrm{GeV}$ ), as $m_{\mathrm{t}}$ increases from 150 to $200 \mathrm{GeV}$. At high luminosity however, as shown in Table 52, the t $\overline{\mathrm{t}}$ background becomes dominant, because of its better acceptance for the higher jet $\mathrm{p}_{\mathrm{T}}$-threshold.

Fig 103 shows the distributions of the larger of the transverse momenta reconstructed for the lepton and the b-jet pair, $\max \left(\mathrm{p}_{\mathrm{T}}{ }^{h}, \mathrm{p}_{\mathrm{T}}{ }^{Z}\right)$, and of the missing transverse energy, $E_{T}^{\text {miss }}$, for the $\mathrm{A} \rightarrow \mathrm{Zh}$ signal and the dominant $\mathrm{Zb} \overline{\mathrm{b}}$ and $\mathrm{t} \overline{\mathrm{t}}$ backgrounds after all cuts. The $\mathrm{Zb} \overline{\mathrm{b}}$ background can be further reduced by requiring that $\max \left(\mathrm{p}_{\mathrm{T}}{ }^{h}, \mathrm{p}_{\mathrm{T}}{ }^{Z}\right)$ be larger than a given threshold,

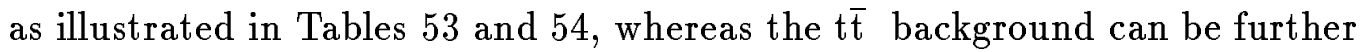
reduced by requiring $E_{T}^{\text {miss }}<60 \mathrm{GeV}$, as illustrated in Tables 55 and 56 . This latter cut was used to obtain the final results for this channel, and Tables 57 and 58 show for completeness the expected signal observability for $\tan \beta=3$ and for the same values of $m_{\mathrm{A}}$, which correspond to $m_{\mathrm{h}}=96,97$ and $98 \mathrm{GeV}$. These larger values of $m_{\mathrm{h}}$ improve the signal acceptance by a factor of $\sim 2$, but result in an even larger increase for the $Z b \bar{b}$ and $t \bar{t}$ backgrounds. These last Tables also show that the expected signal rates decrease very rapidly as $\tan \beta$ increases. 
Table 51: Observability of the $\mathrm{A} \rightarrow \mathrm{Zh} \rightarrow \ell \ell \mathrm{b} \overline{\mathrm{b}}$ channel for an integrated luminosity of $3 \cdot 10^{4} \mathrm{pb}^{-1}$ (low luminosity). The $\sigma \times B R$ values and the expected numbers of signal and background events are given for $m_{\mathrm{h}} \sim 72 \mathrm{GeV}$ $(\tan \beta=1)$ and for three values of $m_{\mathrm{t}}$.

\begin{tabular}{|c||c||c||c|}
\hline$m_{\mathrm{A}}(\mathrm{GeV})$ & 200 & 250 & 300 \\
$m_{\mathrm{h}}(\mathrm{GeV})$ & 71 & 72 & 73 \\
\hline$\sigma \times B R(\mathrm{fb})$ & 561 & 472 & 341 \\
\hline Signal & $770 \pm 19$ & $897 \pm 19$ & $739 \pm 15$ \\
\hline \hline $\mathrm{Zbb}$ & $882 \pm 90$ & $315 \pm 53$ & $135 \pm 35$ \\
\hline $\mathrm{ZZ}$ & $35 \pm 2$ & $16 \pm 2$ & $6 \pm 1$ \\
\hline $\mathrm{Zjj}$ & $19 \pm 1$ & $9 \pm 1$ & $3 \pm 1$ \\
\hline $\mathrm{t} \overline{\mathrm{t}}, m_{\mathrm{t}}=150 \mathrm{GeV}$ & $780 \pm 34$ & $328 \pm 22$ & $101 \pm 12$ \\
$\mathrm{t} \overline{\mathrm{t}}, m_{\mathrm{t}}=175 \mathrm{GeV}$ & $264 \pm 14$ & $184 \pm 12$ & $82 \pm 8$ \\
$\mathrm{t} \overline{\mathrm{t}}, m_{\mathrm{t}}=200 \mathrm{GeV}$ & $77 \pm 6$ & $75 \pm 6$ & $47 \pm 4$ \\
\hline \hline $\mathrm{S} / \sqrt{\mathrm{B}}, m_{\mathrm{t}}=150 \mathrm{GeV}$ & $18.6 \pm 0.7$ & $34.7 \pm 1.7$ & $47.2 \pm 3.7$ \\
$\mathrm{~S} / \sqrt{\mathrm{B}}, m_{\mathrm{t}}=175 \mathrm{GeV}$ & $22.2 \pm 1.0$ & $39.2 \pm 2.2$ & $49.2 \pm 4.0$ \\
$\mathrm{~S} / \sqrt{\mathrm{B}}, m_{\mathrm{t}}=200 \mathrm{GeV}$ & $24.2 \pm 1.2$ & $44.0 \pm 3.0$ & $53.5 \pm 5.0$ \\
\hline
\end{tabular}

Table 52: Observability of the $\mathrm{A} \rightarrow \mathrm{Zh} \rightarrow \ell \ell \mathrm{b} \overline{\mathrm{b}}$ channel for an integrated luminosity of $10^{5} \mathrm{pb}^{-1}$ (high luminosity). The $\sigma \times B R$ values and the expected numbers of signal and background events are given for $m_{\mathrm{h}} \sim 72 \mathrm{GeV}$ $(\tan \beta=1)$ and for three values of $m_{\mathrm{t}}$.

\begin{tabular}{|c||c||c||c|}
\hline$m_{\mathrm{A}}(\mathrm{GeV})$ & 200 & 250 & 300 \\
$m_{\mathrm{h}}(\mathrm{GeV})$ & 71 & 72 & 73 \\
\hline$\sigma \times B R(\mathrm{fb})$ & 561 & 472 & 341 \\
\hline Signal & $397 \pm 21$ & $985 \pm 30$ & $1160 \pm 28$ \\
\hline \hline $\mathrm{Zb \overline {b }}$ & $167 \pm 60$ & $146 \pm 55$ & $21 \pm 21$ \\
\hline $\mathrm{ZZ}$ & $7 \pm 2$ & $11 \pm 2$ & $4 \pm 2$ \\
\hline $\mathrm{Zjj}$ & $16 \pm 3$ & $19 \pm 3$ & $15 \pm 3$ \\
\hline $\mathrm{t} \overline{\mathrm{t}}, m_{\mathrm{t}}=150 \mathrm{GeV}$ & $410 \pm 38$ & $262 \pm 30$ & $58 \pm 15$ \\
$\mathrm{t} \overline{\mathrm{t}}, m_{\mathrm{t}}=175 \mathrm{GeV}$ & $184 \pm 18$ & $228 \pm 20$ & $101 \pm 13$ \\
$\mathrm{t} \overline{\mathrm{t}}, m_{\mathrm{t}}=200 \mathrm{GeV}$ & $55 \pm 7$ & $103 \pm 10$ & $73 \pm 8$ \\
\hline \hline $\mathrm{S} / \sqrt{\mathrm{B}}, m_{\mathrm{t}}=150 \mathrm{GeV}$ & $16.2 \pm 1.3$ & $47.1 \pm 3.7$ & $117.2 \pm 15.5$ \\
$\mathrm{~S} / \sqrt{\mathrm{B}}, m_{\mathrm{t}}=175 \mathrm{GeV}$ & $20.5 \pm 2.1$ & $49.0 \pm 3.9$ & $97.7 \pm 9.0$ \\
$\mathrm{~S} / \sqrt{\mathrm{B}}, m_{\mathrm{t}}=200 \mathrm{GeV}$ & $25.4 \pm 3.8$ & $59.0 \pm 6.2$ & $109.1 \pm 11.4$ \\
\hline
\end{tabular}


Table 53: Observability of the $\mathrm{A} \rightarrow \mathrm{Zh} \rightarrow \ell \ell \mathrm{b} \overline{\mathrm{b}}$ channel for an integrated luminosity of $3 \cdot 10^{4} \mathrm{pb}^{-1}$ (low luminosity). The $\sigma \times B R$ values and the expected numbers of signal and background events are given for $m_{\mathrm{h}} \sim 72 \mathrm{GeV}$ $(\tan \beta=1)$ and for three values of $m_{\mathrm{t}} . A$ cut on $\max \left(p_{\mathrm{T}}{ }^{h}, p_{\mathrm{T}}{ }^{Z}\right)$ is applied (see text).

\begin{tabular}{|c||c||c||c|}
\hline$m_{\mathrm{A}}(\mathrm{GeV})$ & 200 & 250 & 300 \\
$m_{\mathrm{h}}(\mathrm{GeV})$ & 71 & 72 & 73 \\
\hline$\sigma \times B R(\mathrm{fb})$ & 561 & 472 & 341 \\
\hline $\max \left(\mathrm{p}_{\mathrm{T}}^{h}, \mathrm{p}_{\mathrm{T}}{ }^{Z}\right)$ & $>40 \mathrm{GeV}$ & $>80 \mathrm{GeV}$ & $>80 \mathrm{GeV}$ \\
\hline Signal & $706 \pm 18$ & $712 \pm 17$ & $692 \pm 14$ \\
\hline \hline $\mathrm{Zb} \overline{\mathrm{b}}$ & $738 \pm 82$ & $126 \pm 34$ & $90 \pm 28$ \\
\hline $\mathrm{ZZ}$ & $25 \pm 2$ & $8 \pm 1$ & $4 \pm 1$ \\
\hline $\mathrm{Zjj}$ & $11 \pm 1$ & $3 \pm 1$ & $2 \pm 1$ \\
\hline $\mathrm{t} \overline{\mathrm{t}}, m_{\mathrm{t}}=150 \mathrm{GeV}$ & $699 \pm 32$ & $200 \pm 17$ & $86 \pm 11$ \\
$\mathrm{t} \overline{\mathrm{t}}, m_{\mathrm{t}}=175 \mathrm{GeV}$ & $243 \pm 14$ & $144 \pm 10$ & $72 \pm 7$ \\
$\mathrm{t} \overline{\mathrm{t}}, m_{\mathrm{t}}=200 \mathrm{GeV}$ & $75 \pm 6$ & $60 \pm 5$ & $46 \pm 4$ \\
\hline \hline $\mathrm{S} / \sqrt{\mathrm{B}}, m_{\mathrm{t}}=150 \mathrm{GeV}$ & $18.4 \pm 1.0$ & $38.8 \pm 2.4$ & $51.3 \pm 4.4$ \\
$\mathrm{~S} / \sqrt{\mathrm{B}}, m_{\mathrm{t}}=175 \mathrm{GeV}$ & $22.1 \pm 1.0$ & $42.5 \pm 2.8$ & $53.4 \pm 4.8$ \\
$\mathrm{~S} / \sqrt{\mathrm{B}}, m_{\mathrm{t}}=200 \mathrm{GeV}$ & $24.2 \pm 1.3$ & $50.7 \pm 4.6$ & $58.1 \pm 6.0$ \\
\hline
\end{tabular}

Table 54: Observability of the $\mathrm{A} \rightarrow \mathrm{Zh} \rightarrow \ell \ell \mathrm{b} \overline{\mathrm{b}}$ channel for an integrated luminosity of $10^{5} \mathrm{pb}^{-1}$ (high luminosity). The $\sigma \times B R$ values and the expected numbers of signal and background events are given for $m_{\mathrm{h}} \sim 72 \mathrm{GeV}$ $(\tan \beta=1)$ and for three values of $m_{\mathrm{t}} . A$ cut on $\max \left(p_{\mathrm{T}}{ }^{h}, p_{\mathrm{T}}{ }^{Z}\right)$ is applied (see text).

\begin{tabular}{|c||c||c||c|}
\hline$m_{\mathrm{A}}(\mathrm{GeV})$ & 200 & 250 & 300 \\
$m_{\mathrm{h}}(\mathrm{GeV})$ & 71 & 72 & 73 \\
\hline$\sigma \times B R(\mathrm{fb})$ & 561 & 472 & 341 \\
\hline $\max \left(\mathrm{p}_{\mathrm{T}}{ }^{h}, \mathrm{p}_{\mathrm{T}}{ }^{Z}\right)$ & $>50 \mathrm{GeV}$ & $>80 \mathrm{GeV}$ & $>80 \mathrm{GeV}$ \\
\hline Signal & $367 \pm 20$ & $874 \pm 29$ & $1133 \pm 28$ \\
\hline \hline $\mathrm{Zb \overline {b }}$ & $125 \pm 51$ & $104 \pm 46$ & $21 \pm 21$ \\
\hline $\mathrm{ZZ}$ & $5 \pm 2$ & $7 \pm 2$ & $3 \pm 1$ \\
\hline $\mathrm{Zjj}$ & $11 \pm 3$ & $15 \pm 3$ & $13 \pm 3$ \\
\hline $\mathrm{t} \overline{\mathrm{t}}, m_{\mathrm{t}}=150 \mathrm{GeV}$ & $387 \pm 36$ & $207 \pm 27$ & $58 \pm 14$ \\
$\mathrm{t} \overline{\mathrm{t}}, m_{\mathrm{t}}=175 \mathrm{GeV}$ & $172 \pm 18$ & $202 \pm 19$ & $89 \pm 12$ \\
$\mathrm{t} \overline{\mathrm{t}}, m_{\mathrm{t}}=200 \mathrm{GeV}$ & $54 \pm 7$ & $94 \pm 10$ & $71 \pm 8$ \\
\hline \hline $\mathrm{S} / \sqrt{\mathrm{B}}, m_{\mathrm{t}}=150 \mathrm{GeV}$ & $16.0 \pm 1.3$ & $47.9 \pm 4.2$ & $116.2 \pm 15.4$ \\
$\mathrm{~S} / \sqrt{\mathrm{B}}, m_{\mathrm{t}}=175 \mathrm{GeV}$ & $20.7 \pm 2.1$ & $48.3 \pm 4.0$ & $100.9 \pm 10.0$ \\
$\mathrm{~S} / \sqrt{\mathrm{B}}, m_{\mathrm{t}}=200 \mathrm{GeV}$ & $26.3 \pm 3.8$ & $58.9 \pm 6.6$ & $109.0 \pm 11.6$ \\
\hline
\end{tabular}


Table 55: Observability of the $\mathrm{A} \rightarrow \mathrm{Zh} \rightarrow \ell \mathrm{lb} \overline{\mathrm{b}}$ channel for an integrated luminosity of $3 \cdot 10^{4} \mathrm{pb}^{-1}$ (low luminosity). The $\sigma \times B R$ values and the expected numbers of signal and background events are given for $m_{\mathrm{h}} \sim 72 \mathrm{GeV}$ $(\tan \beta=1)$ and for three values of $m_{\mathrm{t}}$. A cut on $E_{T}^{\text {miss }}$ is applied (see text).

\begin{tabular}{|c||c||c||c|}
\hline$m_{\mathrm{A}}(\mathrm{GeV})$ & 200 & 250 & 300 \\
$m_{\mathrm{h}}(\mathrm{GeV})$ & 71 & 72 & 73 \\
\hline$\sigma \times B R(\mathrm{fb})$ & 561 & 472 & 341 \\
\hline & $E_{T}^{\text {miss }}<60 \mathrm{GeV}$ & $E_{T}^{\text {miss }}<60 \mathrm{GeV}$ & $E_{T}^{\text {miss }}<60 \mathrm{GeV}$ \\
\hline Signal & $675 \pm 18$ & $786 \pm 18$ & $642 \pm 14$ \\
\hline \hline $\mathrm{Zbb}$ & $828 \pm 86$ & $315 \pm 50$ & $126 \pm 34$ \\
\hline $\mathrm{ZZ}$ & $33 \pm 3$ & $16 \pm 2$ & $5 \pm 1$ \\
\hline $\mathrm{Zjj}$ & $18 \pm 1$ & $9 \pm 1$ & $3 \pm 1$ \\
\hline $\mathrm{t} \overline{\mathrm{t}}, m_{\mathrm{t}}=150 \mathrm{GeV}$ & $287 \pm 21$ & $185 \pm 17$ & $66 \pm 10$ \\
$\mathrm{t} \overline{\mathrm{t}}, m_{\mathrm{t}}=175 \mathrm{GeV}$ & $90 \pm 8$ & $93 \pm 8$ & $48 \pm 6$ \\
$\mathrm{t \overline { \textrm {t } }}, m_{\mathrm{t}}=200 \mathrm{GeV}$ & $23 \pm 3$ & $30 \pm 5$ & $19 \pm 3$ \\
\hline \hline $\mathrm{S} / \sqrt{\mathrm{B}}, m_{\mathrm{t}}=150 \mathrm{GeV}$ & $19.7 \pm 0.9$ & $34.3 \pm 2.0$ & $45.4 \pm 4.1$ \\
$\mathrm{~S} / \sqrt{\mathrm{B}}, m_{\mathrm{t}}=175 \mathrm{GeV}$ & $21.7 \pm 1.1$ & $37.8 \pm 2.6$ & $47.6 \pm 4.6$ \\
$\mathrm{~S} / \sqrt{\mathrm{B}}, m_{\mathrm{t}}=200 \mathrm{GeV}$ & $22.5 \pm 2.2$ & $40.9 \pm 3.2$ & $51.9 \pm 5.8$ \\
\hline
\end{tabular}

Table 56: Observability of the $\mathrm{A} \rightarrow \mathrm{Zh} \rightarrow \ell \mathrm{lb} \overline{\mathrm{b}}$ channel for an integrated luminosity of $10^{5} \mathrm{pb}^{-1}$ (high luminosity). The $\sigma \times B R$ values and the expected numbers of signal and background events are given for $m_{\mathrm{h}} \sim 72 \mathrm{GeV}$ $(\tan \beta=1)$ and for three values of $m_{\mathrm{t}}$. A cut on $E_{T}^{\text {miss }}$ is applied (see text).

\begin{tabular}{|c||c||c||c|}
\hline$m_{\mathrm{A}}(\mathrm{GeV})$ & 200 & 250 & 300 \\
$m_{\mathrm{h}}(\mathrm{GeV})$ & 71 & 72 & 73 \\
\hline$\sigma \times B R(\mathrm{fb})$ & 561 & 472 & 341 \\
\hline Signal & $E_{T}^{\text {miss }}<60 \mathrm{GeV}$ & $E_{T}^{\text {miss }}<60 \mathrm{GeV}$ & $E_{T}^{\text {miss }}<60 \mathrm{GeV}$ \\
\hline \hline $\mathrm{Zb \overline {b }}$ & $336 \pm 20$ & $840 \pm 28$ & $1000 \pm 26$ \\
\hline $\mathrm{ZZ}$ & $125 \pm 51$ & $125 \pm 51$ & $21 \pm 21$ \\
\hline $\mathrm{Zjj}$ & $7 \pm 2$ & $11 \pm 2$ & $3 \pm 1$ \\
\hline $\mathrm{t} \overline{\mathrm{t}}, m_{\mathrm{t}}=150 \mathrm{GeV}$ & $16 \pm 3$ & $17 \pm 3$ & $14 \pm 3$ \\
$\mathrm{t} \overline{\mathrm{t}}, m_{\mathrm{t}}=150 \mathrm{GeV}$ & $69 \pm 11$ & $162 \pm 4$ & $45 \pm 12$ \\
$\mathrm{t} \overline{\mathrm{t}}, m_{\mathrm{t}}=150 \mathrm{GeV}$ & $19 \pm 4$ & $135 \pm 15$ & $62 \pm 11$ \\
\hline \hline $\mathrm{S} / \sqrt{\mathrm{B}}, m_{\mathrm{t}}=150 \mathrm{GeV}$ & $17.8 \pm 1.8$ & $47.3 \pm 4.5$ & $109.8 \pm 16.5$ \\
$\mathrm{~S} / \sqrt{\mathrm{B}}, m_{\mathrm{t}}=175 \mathrm{GeV}$ & $22.8 \pm 3.0$ & $49.5 \pm 4.9$ & $100.0 \pm 12.0$ \\
$\mathrm{~S} / \sqrt{\mathrm{B}}, m_{\mathrm{t}}=200 \mathrm{GeV}$ & $26.0 \pm 4.3$ & $60.2 \pm 8.2$ & $123.1 \pm 20.6$ \\
\hline
\end{tabular}


Table 57: Observability of the $\mathrm{A} \rightarrow \mathrm{Zh} \rightarrow \ell \mathrm{lb} \overline{\mathrm{b}}$ channel for an integrated luminosity of $3 \cdot 10^{4} \mathrm{pb}^{-1}$ (low luminosity). The $\sigma \times B R$ values and the expected numbers of signal and background events are given for $m_{\mathrm{h}} \sim 97 \mathrm{GeV}$ $(\tan \beta=3)$ and for three values of $m_{\mathrm{t}}$. A cut on $E_{T}^{\mathrm{miss}}$ is applied (see text).

\begin{tabular}{|c||c||c||c|}
\hline$m_{\mathrm{A}}(\mathrm{GeV})$ & 200 & 250 & 300 \\
$m_{\mathrm{h}}(\mathrm{GeV})$ & 96 & 97 & 98 \\
\hline$\sigma \times B R(\mathrm{fb})$ & 9 & 21 & 17 \\
\hline & $E_{T}^{\text {miss }}<60 \mathrm{GeV}$ & $E_{T}^{\text {miss }}<60 \mathrm{GeV}$ & $E_{T}^{\text {miss }}<60 \mathrm{GeV}$ \\
\hline Signal & $15.3 \pm \mathbf{0 . 3}$ & $39 \pm 1$ & $36.7 \pm \mathbf{0 . 7}$ \\
\hline \hline $\mathrm{Zb \overline {b }}$ & $860 \pm 88$ & $702 \pm 80$ & $370 \pm 57$ \\
\hline $\mathrm{ZZ}$ & $50 \pm 3$ & $40 \pm 3$ & $20 \pm 2$ \\
\hline $\mathrm{Zjj}$ & $18 \pm 1$ & $15 \pm 1$ & $8 \pm 1$ \\
\hline $\mathrm{t} \overline{\mathrm{t}}, m_{\mathrm{t}}=150 \mathrm{GeV}$ & $762 \pm 33$ & $803 \pm 34$ & $322 \pm 22$ \\
$\mathrm{t} \overline{\mathrm{t}}, m_{\mathrm{t}}=175 \mathrm{GeV}$ & $215 \pm 12$ & $365 \pm 17$ & $249 \pm 14$ \\
$\mathrm{t} \overline{\mathrm{t}}, m_{\mathrm{t}}=200 \mathrm{GeV}$ & $74 \pm 6$ & $144 \pm 8$ & $130 \pm 7$ \\
\hline $\mathrm{S} / \sqrt{\mathrm{B}}, m_{\mathrm{t}}=150 \mathrm{GeV}$ & $\mathbf{0 . 3 7} \pm \mathbf{0 . 0 1}$ & $\mathbf{0 . 9 9} \pm \mathbf{0 . 0 3}$ & $1.37 \pm \mathbf{0 . 0 6}$ \\
$\mathrm{S} / \sqrt{\mathrm{B}}, m_{\mathrm{t}}=175 \mathrm{GeV}$ & $\mathbf{0 . 4 5} \pm \mathbf{0 . 0 2}$ & $1.16 \pm \mathbf{0 . 0 5}$ & $1.44 \pm \mathbf{0 . 0 7}$ \\
$\mathrm{S} / \sqrt{\mathrm{B}}, m_{\mathrm{t}}=200 \mathrm{GeV}$ & $\mathbf{0 . 4 8 \pm \mathbf { 0 . 0 2 }}$ & $1.30 \pm \mathbf{0 . 0 6}$ & $1.60 \pm \mathbf{0 . 0 9}$ \\
\hline
\end{tabular}

Table 58: Observability of the $\mathrm{A} \rightarrow \mathrm{Zh} \rightarrow \ell \ell \mathrm{b} \overline{\mathrm{b}}$ channel for an integrated luminosity of $3 \cdot 10^{4} \mathrm{pb}^{-1}$ (low luminosity). The $\sigma \times B R$ values and the expected numbers of signal and background events are given for $m_{\mathrm{h}} \sim 97 \mathrm{GeV}$ $(\tan \beta=3)$ and for three values of $m_{\mathrm{t}}$. A cut on $E_{T}^{\text {miss }}$ is applied (see text).

\begin{tabular}{|c||c||c||c|}
\hline$m_{\mathrm{A}}(\mathrm{GeV})$ & 200 & 250 & 300 \\
$m_{\mathrm{h}}(\mathrm{GeV})$ & 96 & 97 & 98 \\
\hline$\sigma \times B R(\mathrm{fb})$ & 9 & 21 & 17 \\
\hline \multicolumn{1}{c||}{} & $E_{T}^{\text {miss }}<60 \mathrm{GeV}$ & $E_{T}^{\text {miss }}<60 \mathrm{GeV}$ & $E_{T}^{\text {miss }}<60 \mathrm{GeV}$ \\
\hline \hline Signal & $12.0 \pm \mathbf{0 . 5}$ & $46.4 \pm 1.4$ & $57.0 \pm 1.4$ \\
\hline \hline $\mathrm{Zb} \overline{\mathrm{b}}$ & $290 \pm 78$ & $375 \pm 88$ & $250 \pm 72$ \\
\hline $\mathrm{Zjj}$ & $34 \pm 4$ & $30 \pm 4$ & $20 \pm 3$ \\
\hline $\mathrm{t} \overline{\mathrm{t}}, m_{\mathrm{t}}=150 \mathrm{GeV}$ & $57 \pm 6$ & $44 \pm 5$ & $34 \pm 5$ \\
$\mathrm{t} \overline{\mathrm{t}}, m_{\mathrm{t}}=175 \mathrm{GeV}$ & $225 \pm 20$ & $724 \pm 50$ & $328 \pm 34$ \\
$\mathrm{t} \overline{\mathrm{t}}, m_{\mathrm{t}}=200 \mathrm{GeV}$ & $89 \pm 9$ & $211 \pm 14$ & $362 \pm 25$ \\
\hline \hline $\mathrm{S} / \sqrt{\mathrm{B}}, m_{\mathrm{t}}=150 \mathrm{GeV}$ & $\mathbf{0 . 3 5} \pm \mathbf{0 . 0 2}$ & $1.35 \pm \mathbf{0 . 0 7}$ & $2.27 \pm \mathbf{0 . 1 5}$ \\
$\mathrm{S} / \sqrt{\mathrm{B}}, m_{\mathrm{t}}=175 \mathrm{GeV}$ & $\mathbf{0 . 4 9} \pm \mathbf{0 . 0 4}$ & $1.54 \pm \mathbf{0 . 0 9}$ & $2.21 \pm \mathbf{0 . 1 4}$ \\
$\mathrm{S} / \sqrt{\mathrm{B}}, m_{\mathrm{t}}=200 \mathrm{GeV}$ & $\mathbf{0 . 5 5} \pm \mathbf{0 . 0 5}$ & $1.81 \pm \mathbf{0 . 1 3}$ & $2.56 \pm \mathbf{0 . 2 0}$ \\
\hline
\end{tabular}

